\title{
Top quark spin correlations and leptonic forward-backward asymmetries at D0
}

\author{
A thesis submitted to the University of Manchester for the degree of \\ Doctor of Philosophy \\ in the Faculty of Engineering and Physical Sciences
}

2012

Timothy Daniel Head

School of Physics and Astronomy 


\section{Contents}

$\begin{array}{lr}\text { Abstract } & 5\end{array}$

$\begin{array}{lr}\text { Declaration } & 6\end{array}$

$\begin{array}{ll}\text { Copyright } & 7\end{array}$

$\begin{array}{llr}1 & \text { Introduction } & 9\end{array}$

2 The Standard Model of Particle Physics 11

2.1 The standard model . . . . . . . . . . . . . . . . . . . 11

2.2 Interaction probabilities . . . . . . . . . . . . . . 14

2.3 Electroweak interactions . . . . . . . . . . . . . . . . 14

2.4 Strong interactions . . . . . . . . . . . . . . . . . . . 16

2.5 The top quark . . . . . . . . . . . . . . . . 17

2.6 Top quark production . . . . . . . . . . . . . . . . . 18

2.7 Top quark decay . . . . . . . . . . . . . . . . . . . . . . . . 19

3 Experimental Apparatus $\quad 21$

3.1 The accelerator chain . . . . . . . . . . . . . . 21

3.1.1 Proton acceleration . . . . . . . . . . . . 21

3.1.2 Anti proton production and acceleration . . . . . . . 22

3.1 .3 The Tevatron . . . . . . . . . . . . . . 22

3.2 The D0 detector . . . . . . . . . . . . . . . . 23

3.2.1 Central tracking detectors . . . . . . . . . . 24

3.2 .2 Calorimeter . . . . . . . . . . . . . . . 26

3.2 .3 Muon spectrometer . . . . . . . . . . . . . 27

3.2.4 Trigger system . . . . . . . . . . . . . . 27

4 Object Reconstruction and Simulation 30

4.1 Object reconstruction algorithms . . . . . . . . . . . . 30

4.1.1 Tracks . . . . . . . . . . . . . . 30

4.1 .2 Electrons ...................... 31 
4.1 .3 Muons . . . . . . . . . . . . . . . . . . . . . . 32

4.1 .4 Jets $\ldots \ldots \ldots \ldots \ldots \ldots \ldots$

4.1.5 Missing transverse energy _. . . . . . . . . . 34

4.2 Simulation and corrections . . . . . . . . . . . . . . 36

5 Multivariate Electron Identification 38

5.1 Boosted Decision Trees . . . . . . . . . . . . . . . . . . . 39

5.2 Samples used in the training . . . . . . . . . . . . . 41

5.2 .1 Real electrons . . . . . . . . . . . . . . . . 41

5.2 .2 Fake electrons . . . . . . . . . . . . . . . . 43

5.3 Input variables and construction of the decision tree . . . . . . 44

5.4 Performance of the BDT . . . . . . . . . . . . 45

6 Event selection $\quad 55$

7 Spin Correlation $\quad 66$

7.1 Observable . . . . . . . . . . . . . . . . . 67

7.2 Reconstruction . . . . . . . . . . . . . . . . . 70

7.3 Template fitting . . . . . . . . . . . . . . . . . . . . . . . . 74

7.4 Feldman-Cousins method . . . . . . . . . . . . . . . 80

7.5 Matrix element based method . . . . . . . . . . . . . . . 82

7.6 Systematic uncertainties . . . . . . . . . . . . . . . . . 85

7.7 Results . . . . . . . . . . . . . . . . . . . . . . . . . 89

8 Forward Backward Asymmetries $\quad 95$

8.1 Observables . . . . . . . . . . . . . . . . . . . . . . 99

8.2 Predictions . . . . . . . . . . . . . . . . . . . . 103

8.3 Background cross check . . . . . . . . . . . . . . . . . . 104

8.4 Acceptance correction . . . . . . . . . . . . . . . . . . . 109

8.5 Top polarisation . . . . . . . . . . . . . . . . . . . . . . 112

8.6 Combination . . . . . . . . . . . . . . . . . . . . . . . 114

8.7 Systematic uncertainties . . . . . . . . . . . . . . . . 116

8.8 Results . . . . . . . . . . . . . . . . . . . . . 118

9 Conclusion 120

A Control plots 123

A.1 Run-IIa dielectron channel . . . . . . . . . . . . . . . . 124

A.2 Run-IIb dielectron channel . . . . . . . . . . . . . . . . 130

A.3 Run-IIa electron-muon channel . . . . . . . . . . . . . 136

A.4 Run-IIb electron-muon channel . . . . . . . . . . . . . . 142

A.5 Run-IIa dimuon channel . . . . . . . . . . . . . . . . 148 
A.6 Run-IIb dimuon channel . . . . . . . . . . . . . . . . . . . . 154

B Electron identification variables $\quad 160$

B.1 Central calorimeter variables . . . . . . . . . . . . . . . . . 160

B.2 Endcap calorimeter variables . . . . . . . . . . . . . 167

Bibliography

173

Total word count: 25939 


\section{Abstract}

Top quark spin correlations and leptonic forward-backward asymmetries at D0

The University of Manchester

Timothy Daniel Head

Doctor of Philosophy

September $28^{\text {th }}, 2012$

The top quark is the heaviest fundamental particle. Its correspondingly short lifetime implies that it will decay before top flavoured hadrons can form. This provides an opportunity to study the properties of a quark without the effects of hadronisation, which is unique in the standard model.

Using data recorded by the D0 experiment at the Fermilab Tevatron collider, and corresponding to $5.4 \mathrm{fb}^{-1}$ of proton-antiproton collisions two analyses of the production and decay mechanism of top quarks are presented in this thesis. In the standard model the directions of the spin of top quarks produced in pairs by the strong interaction are expected to be correlated. In the first analysis, the strength of the correlation is extracted from the angles of the two leptons in the top quark and antiquark rest frames, yielding a correlation strength $C=0.10 \pm 0.45$. This is in agreement with the NLO QCD prediction within two standard deviations, but also in agreement with the no correlation hypothesis.

In the second analysis, forward-backward asymmetries in top quark-antiquark production are measured using the charged leptons from top quark decays. We find that the angular distributions of $\ell^{-}$relative to antiprotons and $\ell^{+}$relative to protons are in agreement with each other. Combining the two distributions and correcting for detector acceptance we obtain the forward-backward asymmetry $A_{\mathrm{FB}}^{\ell}=(5.8 \pm 5.1$ (stat) \pm 1.3 (syst) $) \%$, compared to the standard model prediction of $A_{\mathrm{FB}}^{\ell}$ (predicted $)=(4.7 \pm 0.1) \%$. 


\section{Declaration}

No portion of the work referred to in this thesis has been submitted in support of an application for another degree or qualification of this or any other university or other institution of learning.

Timothy Daniel Head

School of Physics and Astronomy

University of Manchester

Oxford Road

Manchester

M13 9PL

September 2012 


\section{Copyright}

1. The author of this thesis (including any appendices and/or schedules to this thesis) owns certain copyright or related rights in it (the "Copyright") and he has given The University of Manchester certain rights to use such Copyright, including for administrative purposes.

2. Copies of this thesis, either in full or in extracts and whether in hard or electronic copy, may be made only in accordance with the Copyright, Designs and Patents Act 1988 (as amended) and regulations issued under it or, where appropriate, in accordance with licensing agreements which the University has from time to time. This page must form part of any such copies made.

3. The ownership of certain Copyright, patents, designs, trade marks and other intellectual property (the "Intellectual Property") and any reproductions of copyright works in the thesis, for example graphs and tables ("Reproductions"), which may be described in this thesis, may not be owned by the author and my be owned by third parties. Such Intellectual Property and Reproductions cannot and must not be made available for use without the prior written permission of the owner(s) of the relevant Intellectual Property and/or Reproductions.

4. Further information on the conditions under which disclosure, publication and commercialisation of this thesis, the Copyright and any Intellectual Property and/or Reproductions described in it may take place is available in the University IP policy ${ }^{1}$, in any relevant Thesis restriction declarations deposited in the University Library, The University Library's regulations ${ }^{2}$ and in The University's policy on presentation of Theses.

\footnotetext{
${ }^{1}$ http://www.campus.manchester.ac.uk/medialibrary/policies/intellectualproperty.pdf

${ }^{2}$ http://www.manchester.ac.uk/library/aboutus/regulations
} 
The only way of discovering the limits of the possible is to venture a little way past them into the impossible.

- Arthur C. Clarke 


\section{Chapter 1}

\section{Introduction}

The field of particle physics is concerned with finding the Answer to the Ultimate Question of Life, the Universe, and Everything. It is based on the hypothesis that everything in the universe can be understood as a collection of fundamental pieces, or particles, and their interactions. A bottom up approach to modelling macroscopic objects is, however, mathematically intractable. This limits the application of particle physics' model to systems with few constituent particles interacting over very small distances. It might come as a surprise then, to hear that it is this model which successfully describes why the sun shines and what happened in the earliest moments of the history of the universe. It is referred to as the standard model.

With a mass close to that of a gold nucleus the top quark is the heaviest fundamental particle in the standard model. This thesis documents two analyses of the top quark's production and decay mechanisms performed using the large sample of events with two charged leptons and two jets collected by the D0 experiment at the Fermilab Tevatron.

In the standard model the direction in which the spin of a top quark and antiquark point is expected to be correlated when they are produced in pairs. One of the unique properties of the top quark is that its lifetime is so short that it decays before its spin can be affected by the strong interaction. The first analysis presented in this thesis takes advantage of this unique property and measures the degree to which the direction of the spin of top quark and 
antiquark are correlated.

The second analysis studies a further property of the production of top quark pairs. In the standard model there is a small asymmetry between the number of top quarks produced in the forward and backward directions. This asymmetry is probed by analysing the angular distributions of the charged leptons from top quark decays.

In Chapter 2 a brief overview of the standard model, as well as an overview of top quark physics are given. The accelerator chain and D0 detector are introduced in Chapter 3. In Chapter 4 the techniques used for the reconstruction of physics objects in the detector are described. An improved electron identification algorithm is detailed in Chapter 5. A measurement of the degree to which the direction of top quark and anti-quark are correlated is described in Chapter 7. Chapter 8 documents a measurement of forward backward asymmetries in the lepton rapidity distributions. In the final chapter conclusions are drawn from the work discussed in this thesis. 


\section{Chapter 2}

\section{The Standard Model of Particle Physics}

\subsection{The standard model}

The standard model of particle physics forms the basis for our understanding of the interactions between high energy particles. It has been extremely successful at modelling these interactions from low energies up to the highest energies that have been probed by experiments - from roughly a few electronvolts to a few terraelectronvolts. Despite its success it has some shortcomings. The force of gravity does not feature in this standard model, including it remains, at least for now, a dream. While the standard model very precisely describes the interactions of matter and antimatter, the vast majority of the universe, roughly $96 \%$, consists of something that is neither matter nor antimatter, and is therefore beyond the standard model. As a result the standard model is considered to be an effective theory, successfully describing phenomena below a certain energy threshold.

According to the standard model all matter consists of one or several of the twelve elementary fermions, each of which has an antimatter equivalent. There are two types of fermions: quarks and leptons. The twelve fermions are arranged into three families (shown in Table 2.1) consisting of two quarks, a charged and a neutral lepton each. As a rule of thumb the mass of the 
Table 2.1: The elementary particles in the standard model. All of which have been observed, most recently the Higgs boson.

\begin{tabular}{|c|c|c|}
\hline & Fermions & Bosons \\
\hline Quarks & $\left(\begin{array}{l}\mathrm{u} \\
\mathrm{d}\end{array}\right) \quad\left(\begin{array}{l}\mathrm{c} \\
\mathrm{s}\end{array}\right) \quad\left(\begin{array}{l}\mathrm{t} \\
\mathrm{b}\end{array}\right)$ & $\gamma, \mathrm{W}^{ \pm}, \mathrm{Z}$ \\
\hline Leptons & $\left(\begin{array}{c}\mathrm{e} \\
\gamma_{\mathrm{e}}\end{array}\right) \quad\left(\begin{array}{c}\mu \\
\gamma_{\mu}\end{array}\right) \quad\left(\begin{array}{c}\tau \\
\gamma_{\tau}\end{array}\right)$ & $\mathrm{H}, \mathrm{g}$ \\
\hline
\end{tabular}

particles increases from one family to the next. As a result particles of the second and third family are generally unstable and eventually decay to their lighter selfs in the first family. The lightest and most familiar charged lepton is the electron. The muon and $\tau$ lepton are the charged leptons of the other two families. The neutral lepton in each family is referred to as neutrino. They are thought to be very light, in fact, they were assumed to be exactly massless before the phenomenon of neutrino oscillations was observed [43]. The interactions of the neutrino are very limited, making it difficult to detect experimentally. So much so, that in collider experiments its presence is generally inferred on the basis of energy and angular momentum conservation.

The so called up and down quarks are the quarks of the first family. The second family contains the charm and strange quarks, and the third the top and bottom quarks. Two properties of the top quark, the heaviest of them all, are investigated in this thesis. The up or down quark combine to form stable protons and neutrons. Together protons, neutrons and electrons form the building blocks of atoms.

The interactions between the fermions are modelled by force carriers, vector bosons with spin one. The photon mediates the electromagnetic force between all electrically charged particles. The force carriers of the weak interaction are the $\mathrm{W}^{ \pm}$and $\mathrm{Z}$ bosons, which interact with all fermions as they all carry weak isospin charge. The gluon mediates the strong force, the interaction between the particles carrying colour charge. This includes the quarks, but also the gluons themselves. The photon and gluon are massless, whereas the fermions, $\mathrm{W}^{ \pm}$and $\mathrm{Z}$ bosons are not. The masses of the fermions and the $\mathrm{Z}$ boson constitute ten of the 18 parameters of the standard model. 
The fact that the $\mathrm{W}^{ \pm}$and $\mathrm{Z}$ bosons have a mass of $\approx 80 \mathrm{GeV}$ and $\approx 91 \mathrm{GeV}$, respectively, confines their interactions to distances of $\approx 0.02 \mathrm{fm}$. The photon is massless and therefore the range of the electromagnetic force is unlimited. Despite the gluon being massless the range of the strong force is not infinite, as will be explained in more detail later.

The final piece of the standard model is the mechanism of spontaneous electroweak symmetry breaking proposed by Higgs, Brout, Englert, Guralnik, Hagen and Kibble [41, 46, 51]. It provides a mechanism to generate the weak vector boson and fermion masses, whilst allowing the photon to remain massless, as well as introducing a further spin-0, scalar boson to the set of observable particles. The mass of this boson is a free parameter in the standard model. A detailed review can be found in Reference [40]. Searches for this particle have been a focus of activities in the field of particle physics. Culminating in the announcement of the discovery of a Higgs like particle by the ATLAS and CMS collaborations in July 2012 [3, 38].

The standard model is a unified description of the interactions of all particles in terms of gauge theories. The underlying idea being that conserved physical quantities are closely related to invariance of a Lagrangian under a symmetry transformation. A gauge theory is one which is invariant under a set of local transformations. A local transformation depends on space-time coordinates, where as a global transformation does not. To maintain gauge invariance under a local transformation gauge fields have to be introduced, which turn out to represent the force carries. For example, the local gauge transformation of a field $\psi$ associated with the group $\mathrm{U}(1)$ is a complex phase transformation $\psi \rightarrow \phi e^{i \phi(x)}$. The phase $\phi$ depends on the space-time coordinates $x$. Gauge invariance leads to the introduction of a massless vector particle.

The complete set of interactions of the standard model particles is described by the gauge groups

$$
\mathrm{SU}_{\mathrm{C}}(3) \otimes \mathrm{SU}_{\mathrm{L}}(2) \otimes \mathrm{U}_{\mathrm{Y}}(1)
$$

which are the gauge groups of colour, weak isospin and weak hypercharge 

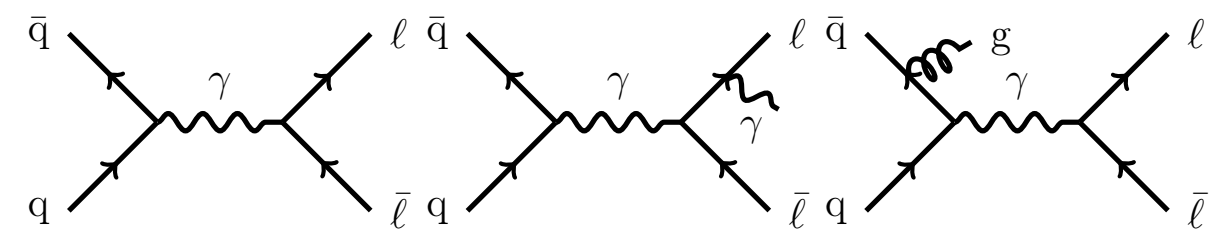

Figure 2.1: On the left the lowest order Feynman diagram of the annihilation of a quark and antiquark to a photon which decays to a lepton and antilepton is shown. The other two diagrams show higher order diagrams which contribute to this process.

respectively. Each will be explained in more detail in the following sections.

\section{$2.2 \quad$ Interaction probabilities}

The method of Feynman diagrams can be used to calculate the probability, or cross section, of a process to occur. The simplest, or lowest order, Feynman diagram showing the annihilation of a quark and antiquark into a lepton and antilepton via the exchange of a photon is shown on the left in Figure 2.1. Each edge and vertex of the graph represents a multiplicative factor in the mathematical expression for the cross section of this process. Also shown are two higher order diagrams for this process, the order of a diagram is related to its number of vertices. By including more diagrams the precision of the calculation increases. Crucially, the calculation of the cross section relies on the coupling constants, the factors associated with a vertex, being small. If this is the case the problem can be treated using perturbation theory, and the series of successive orders converges to a finite value. Otherwise the summation over the infinite number of additional higher order diagrams, which all contribute to the cross section, will result in an infinite cross section.

\section{$2.3 \quad$ Electroweak interactions}

In the standard model the electromagnetic and weak forces are treated in a unified manner, hence the name electroweak interaction. It is described by the $\mathrm{U}_{\mathrm{Y}}(1)$ and $\mathrm{SU}_{\mathrm{L}}(2)$ groups and acts on particles carrying weak hyper- 

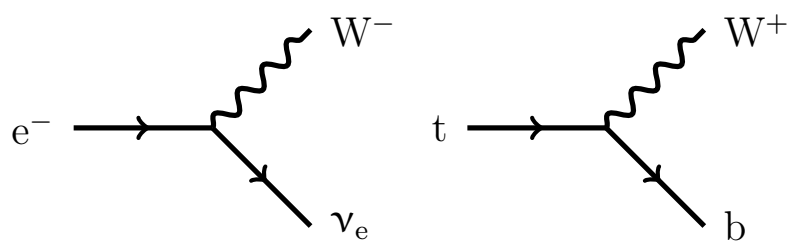

Figure 2.2: The charged current can convert a charged lepton into a neutrino of the same flavour (left). Transitions between up-type and down-type quarks are also possible. On the right the $t \rightarrow \mathrm{W}^{+} \mathrm{b}$ interaction is shown.

charge and isopsin, respectively. The gauge boson of $\mathrm{U}_{\mathrm{Y}}(1)$ is the $\mathrm{B}$ boson, and the $\mathrm{W}^{0}, \mathrm{~W}^{ \pm}$are the gauge bosons of $\mathrm{SU}_{\mathrm{L}}(2)$. The physical $\mathrm{Z}$ boson and the photon $(\gamma)$ are obtained by a rotation from the $W^{0}$ and $B$ bosons:

$$
\left(\begin{array}{l}
\mathrm{Z} \\
\gamma
\end{array}\right)=\left(\begin{array}{cc}
\cos \theta_{W} & \sin \theta_{W} \\
-\sin \theta_{W} & \cos \theta_{W}
\end{array}\right)\left(\begin{array}{c}
\mathrm{W}^{0} \\
\mathrm{~B}
\end{array}\right),
$$

where $\theta_{W}$ is the weak mixing angle. Its value is given by the coupling constants $g$ and $g^{\prime}$ of the $S U(2)_{L}$ and $U(1)_{Y}$ groups, which are two of the 18 parameters of the standard model.

The interactions of the $W^{ \pm}$bosons are referred to as charged current interactions. The charged current can convert a charged lepton into a neutrino of the same family, e.g. $\mathrm{e}^{-} \rightarrow \mathrm{W}^{-} \bar{\nu}_{\mathrm{e}}$. Or, it can convert an up-type quark into a down-type quark, irrespective of the family, e.g. $t \rightarrow \mathrm{W}^{+}$b. Feynman diagrams of both of these processes are shown in Figure 2.2.

Additionally the weak force provides the only mechanism for transitions from one family to another for the quarks. The mixing that occurs between the different families is governed by a $3 \times 3$ unitary matrix $\mathbf{V}_{i j}$, referred to as Cabibbo-Kobayashi-Maskawa matrix [34, 58]. The four numbers required to specify this matrix are parameters of the standard model and have to be determined experimentally. The probability for a transition from quark $i$ to quark $j$ is proportional to $\left|V_{i j}\right|^{2}$. If the matrix $\mathbf{V}_{i j}$ was diagonal then the mass eigenstates would be identical to the eigenstates of the weak interaction, and there would be no mixing. This is however not the case, a review of the measurements and current values of the individual elements can be found in 
reference [26].

The value of $\left|V_{t b}\right|$ can be determined experimentally by comparing branching fractions of the different decays $\mathrm{t} \rightarrow W^{+} q$, with $q=\mathrm{b}, \mathrm{s}$, d. The ratio

$$
R=\frac{\mathcal{B}(\mathrm{t} \rightarrow \mathrm{Wb})}{\mathcal{B}(\mathrm{t} \rightarrow \mathrm{W} q)}=\frac{\left|V_{t b}\right|^{2}}{\sum_{q}\left|V_{t q}\right|^{2}}=\left|V_{t b}\right|^{2}
$$

is measured by counting how many decays of top quarks are deemed to be compatible with a signature corresponding to $\mathrm{t} \rightarrow \mathrm{Wb}$ and the total number of top quark decays. The most recent measurement by D0 finds $0.90<V_{t b}<0.99$ at $95 \%$ C. L. [20]. This method assumes that the CabibboKobayashi-Maskawa matrix is unitary.

Neutral current interactions refer to the interactions of the $\mathrm{Z}$ boson and photon with fermions. The $\mathrm{Z}$ boson couples to fermion-antifermion pairs, while the photon only couples to electrically charged fermion-antifermion pairs. The coupling of the $\mathrm{Z}$ boson to fermions contains both a vector and an axial-vector component, as a result it does not couple exclusively to left handed particles and right handed antiparticles.

Experimentally the interactions of the $\mathrm{W}^{ \pm}$bosons are found to be maximally parity violating. This means that a charged current interaction only takes place between a left handed particle and right handed antiparticle. This property will be useful in the analysis of the spin correlation of top quark pairs.

\subsection{Strong interactions}

Quantum chromodynamics, a non abelian gauge theory based on the $\mathrm{SU}_{\mathrm{C}}(3)$ group describes the strong interaction between particles carrying colour charge. Rotations in the three dimensional colour space are described by eight $3 \times 3$ unitary matrices. The eight gauge bosons associated with the strong force are the called gluons. Unlike the photon, which is electrically neutral, they carry colour charge and as a result can interact with each other.

The coupling constant of quantum chromodynamics $\alpha_{s}$ is a parameter of 
the standard model and varies as a function of the energy of the interaction $Q^{2}$ :

$$
\frac{\partial \alpha_{s}\left(Q^{2}\right)}{\partial \ln \left(Q^{2}\right)}=-\left(11-\frac{2 n_{f}}{3}\right) \frac{\alpha_{s}}{4 \pi}
$$

where $n_{f}$ is the number of fermion families. The dramatic variation of $\alpha_{s}$ leads to a property of quantum chromodynamics known as asymptotic freedom. It is this behaviour of $\alpha_{s}$ which limits the interaction range of the strong force. At low energies, below a couple of gigaelectronvolts, or large distances, $\alpha_{s}$ grows very large and as a result quarks only exist in colourless, bound states, called hadrons.

The large value of the coupling constant means that perturbation theory can not be used. Instead a method called lattice QCD, which is a nonperturbative approach, has to be used to predict the masses of bound states of quarks such as the neutron. Fortunately, at values of $Q^{2}=M_{Z}^{2}$ typical for the production of top quarks the value of $\alpha_{s}$ is roughly $\frac{1}{10}$, and perturbation theory can be used. While the coupling constant of quantum electrodynamics $\alpha_{\mathrm{EW}}$ also varies as a function of energy, the variation is much smaller.

High energy quarks and gluons produced in scattering events radiate other quarks and gluons. Once their energy has reduced so far to make $\alpha_{s}$ large this stops and they form a hadron. This shower of particles is reconstructed and used in detecting quarks and gluons, and is referred to as a jet.

\subsection{The top quark}

The top quark is the most massive known fundamental particle to date. It is the partner of the bottom quark, together they form a weak isospin doublet. In 1995 the CDF [22] and D0 [7] collaborations discovered the top quark at the Tevatron collider. A large effort has gone into measuring its mass, as a result it is the most precisely measured quark mass [1]:

$$
m_{t}=173.2 \pm 0.9 \mathrm{GeV}
$$

With a mass above the mass of the $\mathrm{W}$ boson, the top quark decays rapidly 
to a $\mathrm{W}$ boson and a b quark. The extremely short life time of about $5 \times 10^{-25} \mathrm{~s}$ is so short that the top quark does not form bound states with other quarks. This is unlike all other quarks, and presents the unique opportunity to study a bare quark.

\subsection{Top quark production}

The Tevatron collides protons and antiprotons at a centre-of-mass energy of $\sqrt{s}=1.96 \mathrm{TeV}$. At this energy top quarks are produced predominantly in pairs, referred to as top pair production. The most recent theoretical calculations for the total cross section range from $\sigma_{t \bar{t}} \approx 7 \mathrm{pb}$ to $\approx 8 \mathrm{pb}$. The most recent measurement [11] performed in the same data sample as used in this thesis measures a cross section of:

$$
\sigma_{\mathrm{t} \overline{\mathrm{t}}}=7.36_{-0.79}^{+0.90} \mathrm{pb}
$$

Approximately $85 \%$ of top pairs are produced via the annihilation of a quark and antiquark to a gluon, which then decays to a top quark and antiquark. The remaining $15 \%$ of top pairs is produced by the fusion of two gluons. The leading order Feynman diagrams of both processes are shown in Figure 2.3. The ratio of the two production mechanisms can be understood by considering the typical momentum fraction $x$ of the colliding hadron which is required to produce a pair of top quarks. To produce two top quarks a centre-of-mass energy of at least $2 m_{t}$ is required. At the Tevatron collision energies this means a parton inside the colliding hadron needs to carry at least a fraction $x=\frac{2 \cdot 173.2 \mathrm{GeV}}{1.96 \mathrm{TeV}} \approx 0.18$ of the hadron's momentum. The distribution of the different parton's momentum fraction within a proton or antiproton are referred to as parton density functions (PDF). At $x \approx 0.18$ the ratio of the gluon and quark PDFs corresponds to the ratio of production mechanisms. The ratio of top pairs produced via quark antiquark fusion to gluon gluon fusion will be an important factor in the two analyses presented in this thesis.

The second mechanism for producing top quarks at the Tevatron is single 

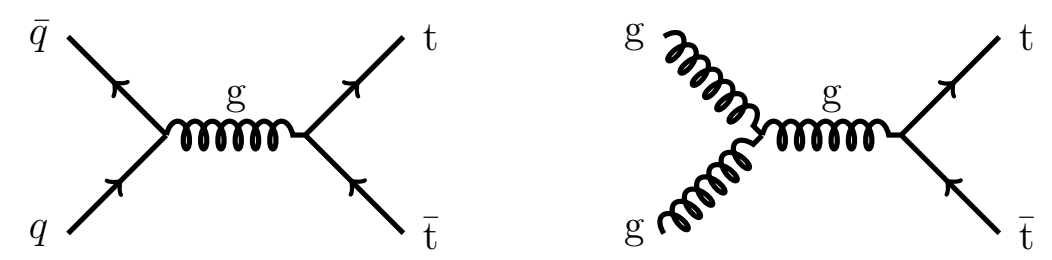

Figure 2.3: Feynman diagrams of the two main production mechanisms for $\mathrm{t} \overline{\mathrm{t}}$ pairs at the Tevatron. On the left $q \bar{q} \rightarrow \mathrm{t} \overline{\mathrm{t}}$ and on the left $g g \rightarrow \mathrm{t} \overline{\mathrm{t}}$.
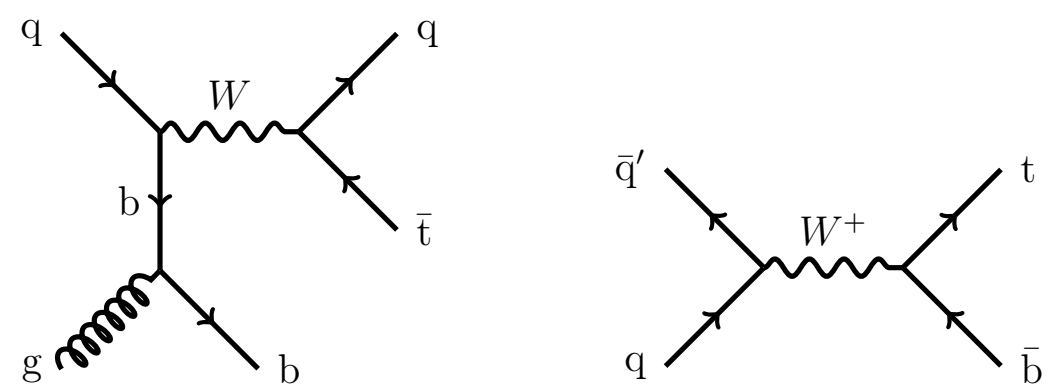

Figure 2.4: Feynman diagrams of single top production at the Tevatron. The $t$-channel, $q g \rightarrow \bar{q}^{\prime} \bar{t} b$, on the left and the $s$-channel diagram, $q \bar{q}^{\prime} \rightarrow t \bar{b}$ on the right.

top production, where only one top quark is produced per event. In Figure 2.4 the two leading order diagrams contributing to this process are shown. The cross section of this process is smaller than for top pairs. The most recent measurement at D0 $\sigma_{t}=3.43_{-0.74}^{+0.73} \mathrm{pb}[12]$ is in good agreement with the theoretical prediction of $3.30 \pm 0.16 \mathrm{pb}$ [57]. Both analyses in this thesis measure properties of top pairs and so single top production is not further considered.

\subsection{Top quark decay}

In the standard model the top quark is predicted to decay to a $\mathrm{W}$ boson and a b quark in nearly $100 \%$ of cases. In general the top quark can decay to any down type quark and $\mathrm{W}$ boson. The rate at which the different decays take place is proportional to the CKM matrix element $\left|V_{t q}\right|^{2}$, with $q=\mathrm{d}, \mathrm{s}, \mathrm{b}$. Under the assumption that there are only three families of quarks, the matrix 
elements $\left|V_{t q}\right|^{2}$ are well constrained, with $V_{t b}>0.999$ [26].

With $V_{t b}$ essentially unity and a mass above $M_{W}$, the top quark decay is dominated by $\mathrm{t} \rightarrow \mathrm{Wb}$. At next-to-leading order the predicted width of the top quark is $1.29 \mathrm{GeV}$ [31], with a correspondingly short lifetime of $5 \times 10^{-25} \mathrm{~s}$. As a result the top quark is not expected to form "toponium" bound states or form top-flavoured hadrons. This unique property means that information about the top quark's spin is reflected in its decay products. This is not the case for lighter quarks, which do form bound states before decaying.

Experimentally, top pair events are separated into three final states according to the decay products of the $\mathrm{W}$ boson. Two types of decays of the $\mathrm{W}$ boson are distinguished. In the first, the $\mathrm{W}$ boson decays leptonically: $\mathrm{W}^{-} \rightarrow \ell_{i} \bar{\nu}_{i}$, where $i=\mathrm{e}, \mu, \tau$ refers to the flavour of the leptons. In the second type, the $\mathrm{W}$ boson decays hadronically: $\mathrm{W}^{-} \rightarrow q \bar{q}^{\prime}$, with $q=\mathrm{u}, \mathrm{c}$ and $\bar{q}^{\prime}=\mathrm{d}$, s, b. The $\mathrm{W}$ boson decays to all these different final states with roughly equal probability, since the masses of the final state particles are much smaller than $M_{W}$. Considering that quarks come in three different colours, two-thirds of W bosons decay hadronically and one-third leptonically.

The final states distinguished experimentally are then the three different possibilities of combining the two decay modes of each of the $\mathrm{W}$ bosons in top pair events. Either, both $\mathrm{W}$ bosons can decay to quarks, $\mathrm{t} \overline{\mathrm{t}} \rightarrow \mathrm{b} q \bar{q}^{\prime} \overline{\mathrm{b}} q^{\prime \prime} \bar{q}^{\prime \prime \prime}$, referred to as the all jets final state. Or, one of the $\mathrm{W}$ bosons decays leptonically and one decays hadronically, $\mathrm{t} \overline{\mathrm{t}} \rightarrow \mathrm{b} q \bar{q}^{\prime} \overline{\mathrm{b}} \ell \bar{\nu}$, the lepton plus jets or $\ell+$ jets final state. Finally, both $\mathrm{W}$ bosons can decay leptonically, $\mathrm{t} \overline{\mathrm{t}} \rightarrow \mathrm{b} \bar{\ell} n u \overline{\mathrm{b}} \ell \bar{\nu}$. This final state is considered in the analyses in this thesis. Experimentally it is challenging to identify $\tau$ leptons, so only decays containing electrons or muons are considered. Considering the branching fraction $\mathcal{B}(W \rightarrow \ell \nu) \approx 32 \%$, about $5 \%$ of all top pairs produced decay to the dilepton final state. 


\section{Chapter 3}

\section{Experimental Apparatus}

\subsection{The accelerator chain}

\subsubsection{Proton acceleration}

The Tevatron collider is the final link in a chain of seven accelerators needed to provide the high energy and quality proton and antiproton collisions for the CDF and D0 detectors. The proton beam, p, starts its life as a collection of humble hydrogen molecules. Using a roughly matchbox sized magnetron ion source. The magnetron consists of an oval cathode surrounded by a anode, with a magnetic field applied across the apparatus. The electric field together with the magnetic field produce an dense plasma. Hydrogen ions are produced by particles colliding with the cathode and releasing hydrogen from the surface.

The acceleration of the the $\mathrm{H}^{-}$ions starts with a $750 \mathrm{kV}$ Cockrof-Walton, followed by a $150 \mathrm{~m}$ long linear accelerator (linac) which they leave with an energy of $400 \mathrm{MeV}$. Between the linac and the Booster the $\mathrm{H}^{-}$ions pass through a carbon foil which removes the electrons thereby creating a beam of protons. The Booster accelerates the protons to an energy of $8 \mathrm{GeV}$ and they reach a speed of $0.99 \mathrm{c}$. Further stages of the accelerator chain will keep increasing the kinetic energy of the protons, however their speed will not increase any further.

Protons are extracted from the Booster and transferred into the Main 
Injector. Here they are accelerated further to $150 \mathrm{GeV}$ ready for injection into the Tevatron.

\subsubsection{Anti proton production and acceleration}

The antiproton, $\overline{\mathrm{p}}$, beam begins life in the same way as the proton beam. However, once extracted from the Booster the protons are accelerated to $120 \mathrm{GeV}$ and then directed towards a fixed Nickel target. The p-Ni collisions produce a large number of particles, including $\bar{p} s$, with a large angular spread, which are focused using a Lithium lens. Only about one in $10^{-5}$ of the produced particles is a $\bar{p}$, these are separated from the other particles by a magnetic charge-mass spectrometer.

Antiprotons are produced in pulses separated by $\approx 1.5 \mathrm{~s}$ and a large spread in momentum. The Debuncher is a triangular accelerator used to trade the small spread in time of the $\overline{\mathrm{p}} \mathrm{s}$ to decrease their spread in energy. This is achieved by having higher energy $\bar{p}$ s travel on a longer orbit and lower energy $\overline{\mathrm{p}} \mathrm{s}$ on a shorter orbit. As a result they see different phases of the accelerating radio-frequency $(\mathrm{RF})$ field.

To make room for the next pulse of $\overline{\mathrm{p}} \mathrm{s}$ the antiprotons are moved to the Accumulator. Here $\bar{p} s$ are stacked over a period of a few hours. The method of "stochastic cooling" is used to further reduce the spread in energy and transverse size of the beam [63]. Periodically the $\overline{\mathrm{p}} \mathrm{s}$ are moved to the Recycler located above the Main Injector. Before injection to the Tevatron the $\overline{\mathrm{p}} \mathrm{s}$ are accelerated to $150 \mathrm{GeV}$ in the Main Injector.

\subsubsection{The Tevatron}

The Tevatron is a super conducting synchrotron which accelerates particles to their final energy of $980 \mathrm{GeV}$. The Tevatron lattice consists of roughly 1000 dipoles, quadrupoles and higher order magnets. The magnets are all cooled by liquid helium.

The beam of protons and antiprotons circulate in opposite directions. Each beam consists of 36 bunches separated by $396 \mathrm{~ns}$, roughly a factor of ten shorter than during Run I. The smaller bunch spacing results in a significant 


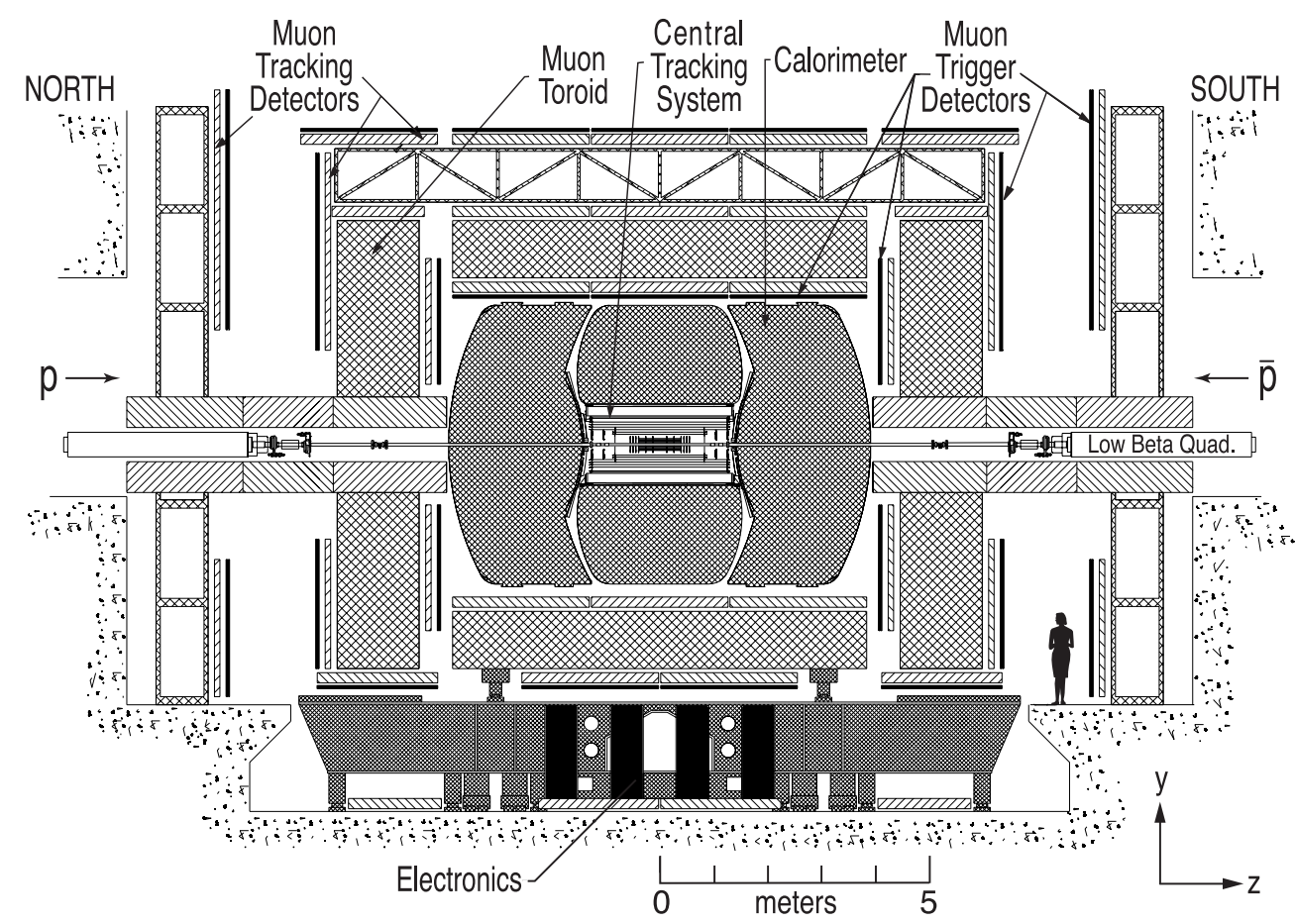

Figure 3.1: Diagram of the D0 detector. Taken from Ref. [18].

increase in the instantaneous luminosity, but presented a challenge for the readout of the calorimeter of the D0 detector.

The dipole magnets operate at $4.2 \mathrm{~T}$ and maintain the circular orbit. At the two collision points quadrupole magnets are used to focus the beams so they collide in a narrow region at the centre of the detectors.

\subsection{The D0 detector}

The D0 detector was designed to study high mass states and large transverse momentum phenomena such as top quark production. Top quark decays involve charged leptons, neutrinos and b quarks which form jets. This means a detector like D0 is ideally suited to study these decays using central tracking detectors surrounded by a liquid-argon/uranium calorimeter and a muon spectrometer. A side on view of the detector as installed is shown in Fig. 3.1 and a detailed description of the detector can be found in Ref [18].

The coordinate system is right handed. The positive $z$ direction points 
along the proton beam and the $y$-axis points upwards. The angle $\theta$ is the polar angle. The azimuthal angle $\phi$ is zero along the $x$-axis and $\phi=\frac{\pi}{2}$ coincides with the $y$-axis. The pseudorapidity is defined as $\eta=-\log [\tan (\theta / 2)]$. The region of $|\eta|<1$ is referred to as "central" and $|\eta|>1$ as "forward".

\subsubsection{Central tracking detectors}

Excellent tracking in the central region is a necessity for top quark studies. The tracking detectors in D0 provide a measurement of the momentum of charged particles and enable finding displaced vertices used in the identification of b-jets. The central tracking system consists of the silicon microstrip tracker (SMT) surrounded by the central fibre tracker (CFT). Both are contained within a $2 \mathrm{~T}$ solenoidal magnetic field created by a superconducting magnet. The layout of inner detector region is shown in Figure 3.2.

The size of the luminous region $(\sigma \approx 25 \mathrm{~cm})$ provides a challenge for the design of the SMT. A "barrel and disk" design was chosen which allows the SMT to have detector surfaces perpendicular to tracks for all values of $\eta$. The detector consists of six barrels and 16 disks. Vertices for particles at at high $|\eta|$ are reconstructed primarily using the $r-z$ measurements of the disks, where as the $r-\phi$ measurements of the barrels are used for central vertices. To compensate for losses in efficiency due to radiation damage of the innermost layer of the SMT the detector was upgraded in 2006 with a new layer of silicon mounted directly on the beam pipe. This is called layer 0 and described in detail in Ref. [25]. The two forward most disks were removed during this upgrade.

The CFT consists of eight cylinders of fibres, each cylinder containing two doublet layers of scintillating fibres. One of the doublet layers is orientated along the beam direction and the second at an alternating stereo angle in $\phi$ of $+3^{\circ}$ and $-3^{\circ}$. The scintillating fibres are connected to visible light photon counters (VLPCs) which are located below the detector by clear fibre waveguides. As a result the waveguides used to read out channels at $\phi=\pi / 2$ are longer than those used at $\phi=3 \pi / 2$. This introduces a $\phi$ dependency into the tracking efficiency. 


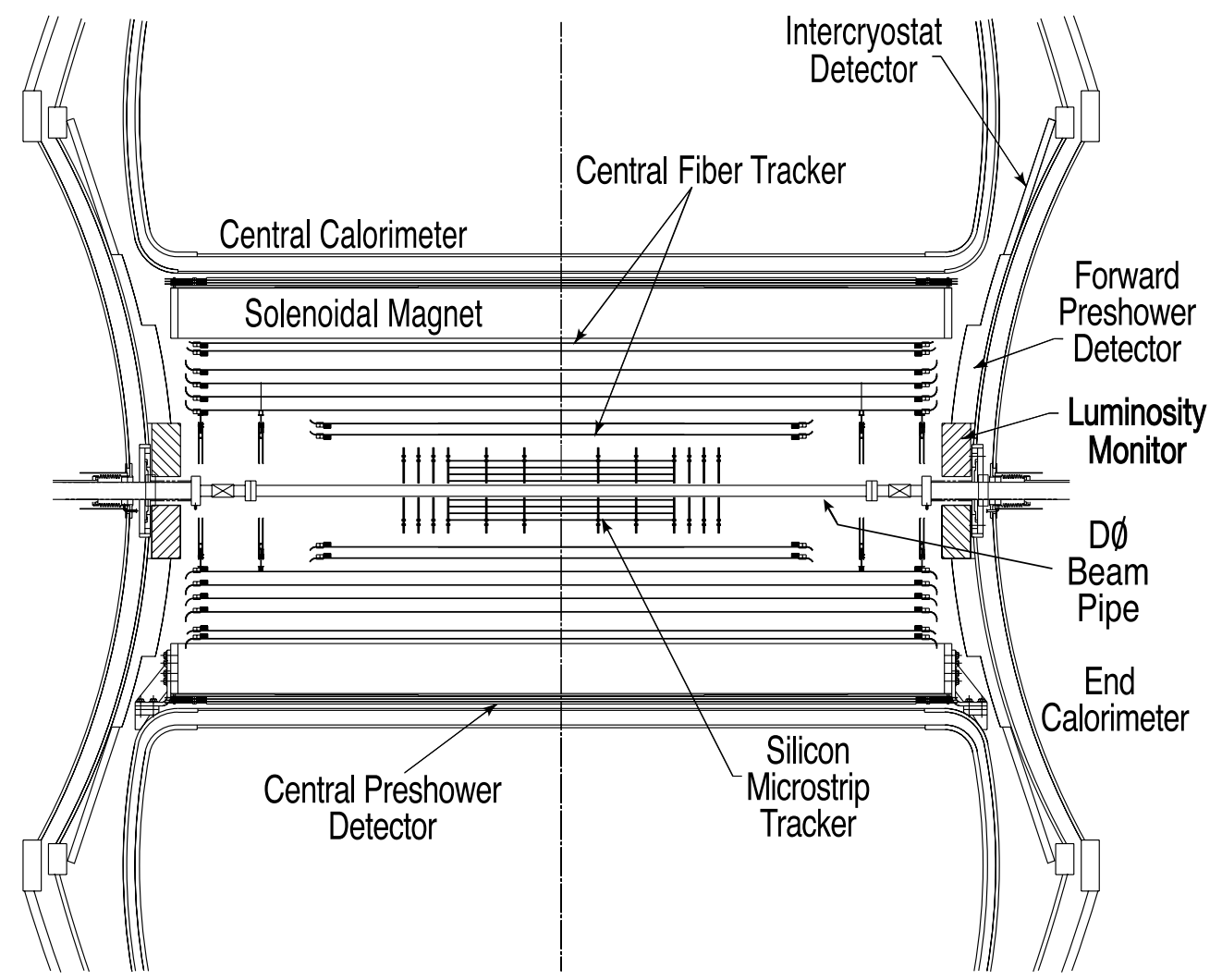

Figure 3.2: A cross-sectional view of the layout of the inner detector region in the $x-z$ plane. The silicon microstrip tracker surrounds the beryllium beam pipe, followed by the central fibre tracker. The solenoidal magnet between the tracking system and calorimeter marks the end of the inner detector. Taken from Ref. [18]. 


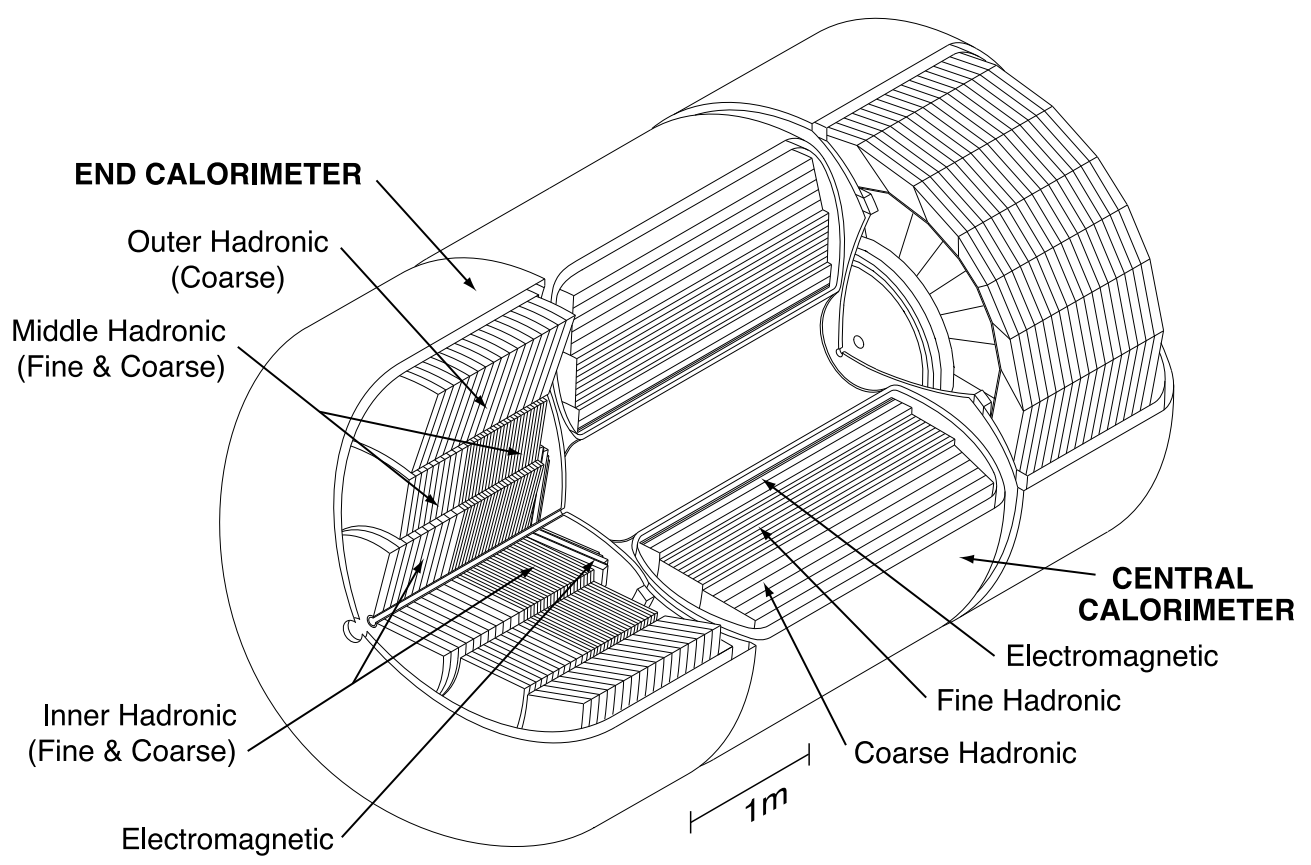

Figure 3.3: Isometric view of the central and two endcap calorimeters. Taken from Ref. [18].

To increase the efficiency of separating electrons from photons preshower detectors are used. The central preshower detector (CPS) is mounted between the magnet and the calorimeter and covers $|\eta|<1.3$. The forward preshower detectors (FPS) are mounted before the endcap calorimeter and cover $1.5<$ $|\eta|<2.5$. Like the CFT they are read out by VLPCs.

\subsubsection{Calorimeter}

The D0 calorimeter is shown in Figure 3.3. The calorimeter consists of three sampling calorimeters. The central calorimeter covers $|\eta| \lesssim 1$ and the two endcap calorimeters provide coverage up to $|\eta| \lesssim 4$. Each calorimeter consists of three sections: electromagnetic (EM), fine hadronic (FH) and coarse hadronic $(\mathrm{CH})$. The electromagnetic section is closest to the interaction region.

Calorimeter cells are arranged into "towers" along lines of constant $\eta$. Each cell consists of alternating layers of absorber plates and signal boards 
covered with a resistive surface. The absorber plates in the EM section are made from nearly pure depleted uranium. The FH sections use a uraniumniobium alloy. In the $\mathrm{CH}$ section copper plates are used in the central region and stainless steel in the forward regions. The active medium of the calorimeter is liquid argon which fills the gaps between the plates. A voltage of $2 \mathrm{kV}$ is applied across the gap between a copper and uranium plate. Cells generally have a size of $\Delta \eta \times \Delta \phi=0.1 \times 0.1$. The third layer of the EM section corresponds to the shower maximum for electrons and photons. Here cells have a higher granularity of $\Delta \eta \times \Delta \phi=0.05 \times 0.05$.

\subsubsection{Muon spectrometer}

The muon spectrometer consists of two types of detectors: drift tubes and scintillators. A toroidal magnetic field allows for a momentum measurement which is independent of the central tracking system. In the central region proportional drift tubes (PDTs) are used, where as in the forward region so called mini drift tubes (MDTs) are used. The scintillation counters are used for triggering and the wire chamber based detectors both for triggering and measurement.

The muon system consists of three layers (A, B, C). Each layer is made up of a set of drift tubes and a layer of scintillators. The first layer (A) is located inside and the other two (B, C) are located outside of the toroidal magnet. A detailed view of the muon system is shown in Figure 3.4.

\subsubsection{Trigger system}

The Tevatron beam consists of 36 bunches and completes one turn in $21 \mu \mathrm{s}$ - This means there are about 1.7 million opportunities per second for an interesting collision to occur. It is not possible to record events at such a large rate. Fortunately most collisions are not of interest to analyses performed at D0. The trigger system is used to reduce the event rate by picking out those collisions which are of interest. The system is split into three levels, the

first level (Level 1) has to arrive at a decision in $132 \mathrm{~ns}$, as a result each part of the detector decides independently whether to accept or reject an event. 


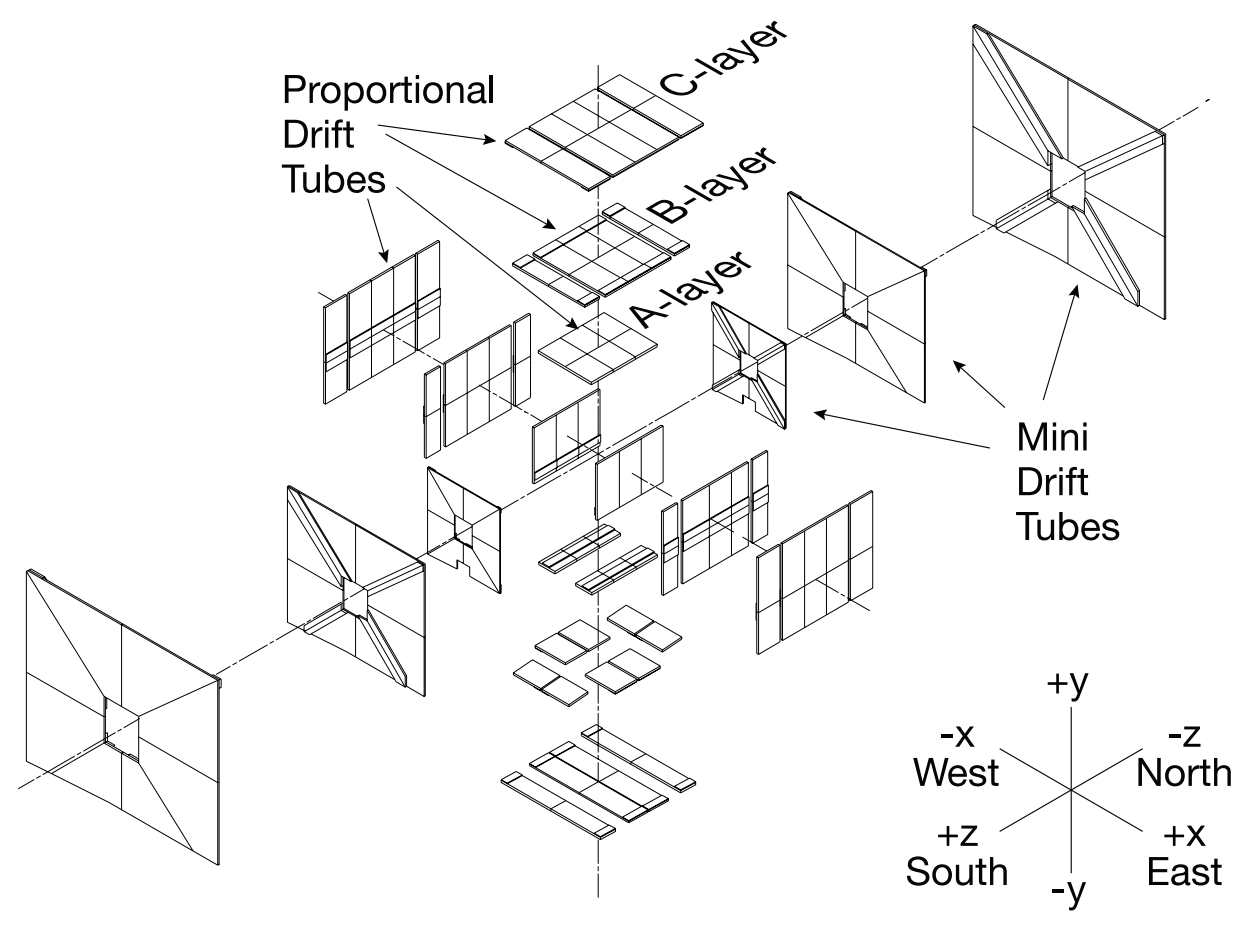

(a) Drift tube layers.

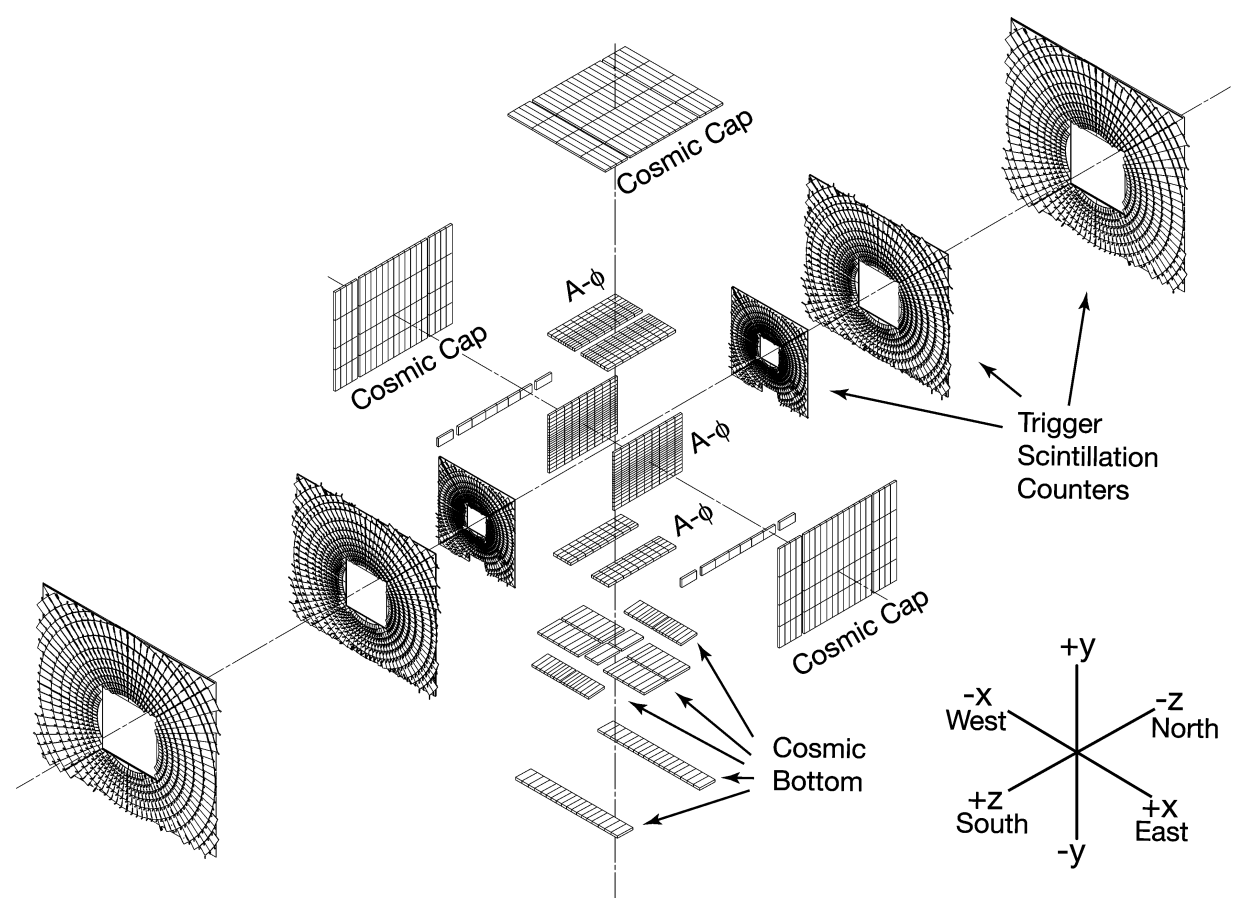

(b) Scintillator layers.

Figure 3.4: Exploded view of the muon system. The muon system consists of three layers, each consisting of a section of scintillators and drift tubes. The drift tube sections are shown at the top and the scintillator sections at the bottom. Taken from Ref. [18]. 28 
More sophisticated decisions are made at the third level (Level 3) where a simplified version of the offline event reconstruction algorithms are run on the full detector read out in the $150 \mathrm{~ms}$ which are available.

The Level 1 trigger uses fixed latency algorithms implemented using Field Programmable Gate Arrays and reduces the event rate to $2 \mathrm{kHz}$. The Central Track Trigger (CTT) matches pre-programmed patterns to hits in the CFT, CPS and FPS in order to detect the passage of charged particles. The Level-1 calorimeter trigger (L1Cal) combines $2 \times 2$ cells of the calorimeter to form trigger towers and then searches for towers exceeding predetermined $E_{T}$ thresholds. In the muon system track stubs are formed from wire and scintillator hits by the L1Muon trigger. Information from the CTT is used to try and match track stubs to tracks found in the CFT.

The Level 2 trigger systems combine information from different regions of the detector and refine the energy and momentum measurements in order to make a more sophisticated decision. Information from the SMT is combined with hits from the CFT. This allows the silicon track trigger to identify longlived particles through their larger distance of closest approach to the beam axis. The event rate is reduced to $1 \mathrm{kHz}$.

The final stage of the trigger decision is Level 3. It consists of a cluster of off-the-shelf computers and runs a simplified version of the offline event reconstruction. This allows for trigger decisions nearly as sophisticated as used during offline analyses. The event rate is reduced to around $100 \mathrm{~Hz}$ and events accepted by Level 3 are stored permanently for later analysis. 


\section{Chapter 4}

\section{Object Reconstruction and Simulation}

\subsection{Object reconstruction algorithms}

\subsubsection{Tracks}

As a charged particles traverses the tracking detectors it interacts with the active material of them. These interactions are detected and referred to as hits. The trajectory of the particle is reconstructed by combining these hits in a track [56]. The track of charged particle in a solenoidal magnetic field can be described by five parameters:

- the curvature $\frac{q}{p_{T}}$,

- the angle made with a plane perpendicular to the magnetic field $\tan \lambda$,

- the radial distance $r$ at the closest approach to the origin,

- the $z$ coordinate of the at the closest approach to the origin, and

- the angle $\phi$ at the closest approach to the origin.

Tracks are not used as individual objects but are used as part of the reconstruction of electrons, jets and muons. 


\subsubsection{Electrons}

Photons and electrons interact with the dense material of the first layers of the calorimeter in the form of electromagnetic showers. Forming clusters from isolated deposits in the calorimeter is the first step of the reconstruction of electrons and photons. The clustering is performed by combining cells within a cone of radius $\Delta \mathcal{R}=\sqrt{(\Delta \phi)^{2}+(\Delta \eta)^{2}}=0.2$ centred on seed cells. The seed cells are found by searching for individual cells above a certain threshold.

The next step is to attempt to match a track to this cluster, if successful the cluster is considered as an electron. If no matching track can be found the cluster is assumed to be a photon.

A hadronic jet containing charged tracks and an overlapping $\pi^{0} \rightarrow \gamma \gamma$ can be mistakenly be identified as an isolated electron. While the probability of this is very low, the rate of jet production is large, leading to the major source of background. In order to distinguish jets from real electrons a combination of several variables describing the properties of the shower and track are used:

- Considering the distribution of energy deposited in the calorimeter, electrons will be contained within the EM layers. Whereas jets will deposit a large amount of energy in the hadronic section of the calorimeter. The fraction of the cluster's energy in the EM layer $\mathcal{F}_{\mathrm{EM}}$ tends to be close to one for electrons, and lower jet jets.

- The $\chi_{\mathrm{EM}}^{2(7)}$ and $\chi_{\mathrm{EM}}^{2(8)}$ variables combine information about the longitudinal and lateral shape of the energy deposits in the EM layers [21].

- EM clusters from real electrons are expected to be isolated from other activity in the calorimeter. The calorimeter isolation is defined as:

$$
\mathcal{I}_{\text {cal }}=\frac{E_{T}^{\mathrm{tot}}(0.4)-E_{T}^{\mathrm{EM}}(0.2)}{E_{T}^{\mathrm{EM}}(0.2)}
$$

where $E_{T}^{\text {tot }}(\Delta \mathcal{R})$ is the transverse energy deposited in all layers of the calorimeter within a cone of radius $\Delta \mathcal{R}$ and $E_{T}^{\mathrm{EM}}(\Delta \mathcal{R})$ is limited to cells in the EM layers. 
- Similar to the calorimeter isolation, the track isolation is calculated as the sum of the $p_{T}$ of all reconstructed tracks within a cone of $0.05<$ $\Delta \mathcal{R}<0.4$ around the electron. A hollow cone is used in order to exclude the track belonging to the candidate electron.

- If a candidate electron is matched to a central track the ratio $\frac{E_{\text {cal }}}{p_{\text {trk }}}$ is expected to be close to unity for real electrons. The momentum of the track should be a good estimate of the energy deposited in the EM layers of the calorimeter. Charged hadrons will deposit only a small amount of their energy in the EM layers and so will have a smaller value of $\frac{E_{\text {cal }}}{p_{\text {trk }}}$. Decays of $\pi^{0} \rightarrow \gamma \gamma$ will have a larger value as a random track is more likely to have low $p_{T}$.

- A likelihood discriminant $\mathcal{L}^{8}$ constructed from eight tracking variables and shower shape information.

A new approach to electron identification based on a Boosted Decision Tree (BDT) is described in Section 5. By taking into account the correlation between variables and training separate BDTs for different regions of the detector the efficiency of identifying electrons is improved while keeping the rate at which hadronic jets are misidentified constant.

In both analyses in this thesis electrons are required to satisfy the following criteria:

- $\mathcal{F}_{\mathrm{EM}}>0.9$,

- $\mathcal{I}_{\text {cal }}<0.2$,

- $\chi_{\mathrm{EM}}^{2(7)}<50$ and $\chi_{\mathrm{EM}}^{2(8)}<50$,

- $\frac{E_{\mathrm{cal}}}{p_{\text {trk }}}<2.5$

- $\mathcal{L}^{8}>0.85$.

\subsubsection{Muons}

The distinguishing feature of muons is that they penetrate the calorimeter and leave hits in the muon system. The first step of muon reconstruction is 
to form track segments from the hits in the three layers of the muon system. These are then combined to form a local muon. An attempt is then made to match these local muons to central tracks. By combining central tracks with local muons we obtain a precise estimate of the muon momentum from the former and unambiguous identification from the latter.

Local muon candidates are separated into three categories: loose, medium and tight depending on their number of scintillator and wire hits [35]. In addition to being separated from jets by at least $\mathcal{R}=0.5$, muons must satisfy the following isolation requirements:

- in the calorimeter, the sum over all cells in the calorimeter within a hollow cone of $0.1<\Delta \mathcal{R}<0.4$ around the muon candidate, divided by the $p_{T}$ of the muon candidate,

- in the central tracker, the sum of the $p_{T}$ of all tracks in a cone of $\Delta \mathcal{R}=0.5$ around the muon candidate

Both quantities are required to be $<0.15$ for muons used in this analysis.

\subsubsection{Jets}

Hadronic jets are reconstructed from energy deposits in the calorimeter. Quarks and gluons hadronise and form hadrons and mesons. These interact with the dense material of the calorimeter, and depositing all their energy in it. These energy deposits are referred to as showers. The algorithm used at D0 to associate different energy deposits to an individual jet is a seed based mid-point cone algorithm with a cone size of $\Delta \mathcal{R}=0.5$ [32].

Jets have to satisfy several quality criteria. To distinguish them from electrons $\mathcal{F}_{\mathrm{EM}}$ is required to be below 0.95 , but above 0.05 in order to remove noise. To remove jets clustered around a single cell which fired because of noise the ratio of the energy in the highest energy cell of a jet and the second highest energy cell has to be below 0.1. Furthermore no single cell of a jet is allowed to contain more than $90 \%$ of its energy. The full details of the jet requirements are given in Reference [49]. As the instantaneous luminosity increases, the chance of several proton-antiproton collisions occurring in one 
bunch crossing increases as well. Already at moderate instantaneous luminosities more than one collision takes place per bunch crossing. The results of both collisions are overlaid in the detector, which is sometimes referred to as pile up. To remove jets which arise from this, jets are required to contain at least two tracks associated with the primary vertex. This is referred to as a jet being vertex confirmed.

An important part of jet reconstruction is the calibration of the jet energy scale. The goal of this calibration is to provide a method to correct the energy measured in the calorimeter back to that of the stable particles before they interacted with the D0 detector. In Figure 4.1 the evolution of a parton created in the hard scatter process to an energy deposit in the calorimeter is shown. A full description of the procedure is given in Reference [55].

\subsubsection{Missing transverse energy}

The overall momentum of the collision is zero, protons and antiprotons are circulating in opposite directions, have equal masses and energy. However, after the collision remnants of the proton and antiproton continue down the beam pipe undetected. As a result the sum of the momenta of the detected particles in the $z$ direction will not balance. In the transverse plane, however, the momentum is zero. We make use of this to infer the presence of neutrinos, which otherwise escape without detection.

The calculation of the missing transverse energy, $\mathbb{E}_{T}$, starts with the vectorial sum of the $E_{T}$ of all calorimeter cells. Usually the cells from the coarse hadronic calorimeter are not included in this sum as they contribute a significant amount of noise.

The basic sum of cell $E_{T} \mathrm{~s}$ is corrected for other objects reconstructed in the event. Including photons, electrons, muons and jets. For these objects the energy estimate obtained from the object reconstruction is more accurate than the sum of calorimeter cells associated with those objects. The cells associated with the objects are removed from the sum. 


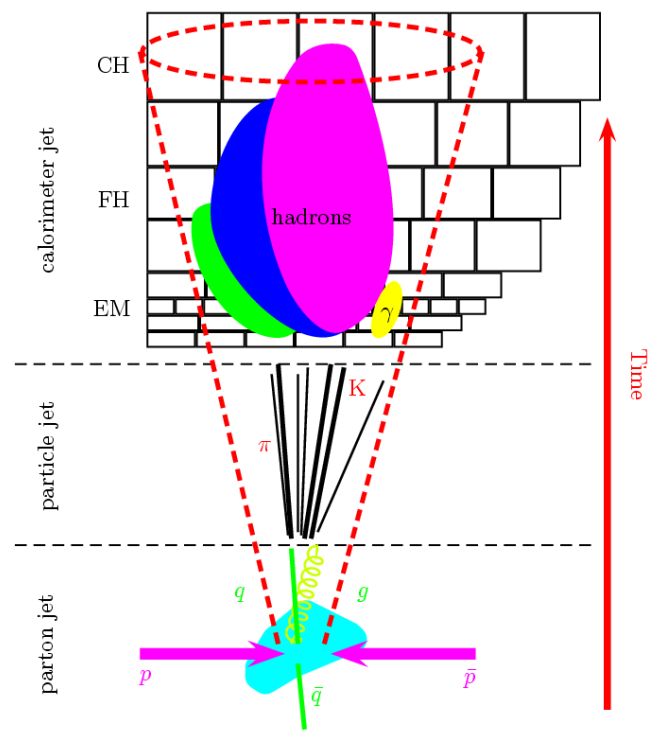

Figure 4.1: The evolution of a parton to a calorimeter shower. Taken from Reference [54]. 


\subsection{Simulation and corrections}

Both analyses described in this thesis rely heavily on simulation. It is used to estimate the effects the detector has on the observables, in addition to predicting background contributions. The first step is the simulation of the physical processes using perturbative QCD. The computer programs used for this are known as Monte Carlo event generators. The name derives from the fact that they commonly use Monte Carlo techniques to perform the integration of the differential cross section of the process they are simulating. Details of which Monte Carlo event generators are used will be given in Section 6.

The Monte Carlo event generator also takes care of simulating the decay of unstable and hadronisation of coloured particles produced in the hard scatter interaction. The stable particles at the end of this simulation step are fed into a GEANT based computer program which simulates the interaction of these particles with the detector itself. The outputs are digitised using the same format as used by the real detector readout. In the digitisation step an event triggered randomly during collisions is merged, or overlaid with the simulated event. The pool of events used for this procedure is referred to as the zero bias overlay. This simulates the effects of additional $p \bar{p}$ interactions in the same bunch crossing. The digitised output is then reconstructed by the same software used to reconstruct real data.

When compared to the data the simulation is found to be overly optimistic with regards to the electron and muon $p_{T}$ resolution and particle identification efficiencies. This is mainly due to an overestimate of the single hit efficiency in the tracking detectors by the simulation. These deficiencies are corrected for by measuring the identification efficiency in data and simulation and applying a weight to the simulated events based on the ratio of the two efficiencies. This event weight is parameterised as a function of the detector $\eta$ and $\phi$ coordinates.

The instantaneous luminosity assigned to an event is based on the instantaneous luminosity of the event from the zero bias overlay which is merged with the event. The overlay events are generally recorded at the beginning 
of a run period, and not continuously updated as the run progresses. Therefore the distribution of instantaneous luminosities represented in the overlay does not reflect the instantaneous luminosity of the recorded data. An event weight is applied to the simulated events which improves the agreement between the instantaneous luminosity profile in data and simulation.

In the simulation the luminous region is modelled as a gaussian distribution of width $25 \mathrm{~cm}$ in the $z$ direction. The real luminous region is much wider $\approx 60 \mathrm{~cm}$ and this width depends on, for example, the instantaneous luminosity. A weight based on the ratio of the distributions of the $z$ coordinate of the primary vertex in data and simulation is applied to the simulated events.

The simulation of the D0 detector does not include a simulation of the triggers used to record data. The efficiency of the individual triggers is measured in data and a weight is applied to the simulation to represent the probability that this event would have been recorded.

There are also known imperfections in the modelling of the hard scattering process. For example the ALPGEN simulation used at D0 does not accurately model the $p_{T}$ distribution of $W$ and $Z$ bosons. A correction in the form of weights is applied to the simulated events. This reweighting is derived from $Z \rightarrow e^{+} e^{-}$events in data. 


\section{Chapter 5}

\section{Multivariate Electron Identification}

Efficiently identifying electrons, while maintaining a low misidentification rate, is crucial for all physics analyses containing electrons in their final state. For example, a measurement of the cross section of the rare diboson processes WW and WZ in a final state containing respectively two or three electrons benefits directly from efficiency improvements in the electron identification [14]. In searches for the standard model Higgs boson in final states with electrons, such as $\mathrm{H} \rightarrow \mathrm{WW} \rightarrow \mathrm{e} \nu \mathrm{e} \nu[16]$ and the associated production of a Higgs and W boson [17], the experimental sensitivity is a direct function of the electron identification efficiency.

In this chapter a novel electron identification algorithm based on a multivariate discriminant is presented. A multivariate discriminator gathers information from several variables, combining them into one output variable. This variable presents the optimal separation between signal and background processes. All electron identification algorithms used at the D0 experiment so far are based on imposing a set of requirements on a combination of the variables described in Section 4.1.2. By employing a Boosted Decision Tree, a type of multivariate discriminator, more complex cuts than simple rectangular cuts are used and the correlations between variables are taken into account. Therefore it is possible to increase the efficiency of identifying 
electrons while keeping the rate at which jets or photons are mistakenly identified as electrons constant compared to the standard rectangular cuts. The first section of this chapter will describe the principles underlying a Boosted Decision Tree.

In order to construct a multivariate discriminant a sample of both signal like and background like events is required. These are used to "learn" the discriminating power of the individual variables, and their dependencies on each other. Here a sample of real electrons and a sample of jets is used to represent the signal and background like events. As there are known deficiencies in the simulation of the D0 detector, both are selected from data in order to present the multivariate discriminator with the correct correlations and dependencies of the variables. How these samples are obtained is described in the second section of this chapter.

In the third section the input variables and the construction of the Boosted Decision Tree are discussed. The final section will compare the performance to the traditional electron identification.

\subsection{Boosted Decision Trees}

Machine learning algorithms have become an everyday tool in high energy physics and are regularly used in order to extract the maximum information from a set of variables in searches for rare phenomena. A Boosted Decision Tree is a supervised machine learning algorithm. The "supervised" means the events used during the construction of the classifier have to be labeled as either signal or background like.

A simple decision tree takes the idea of placing cuts on individual variables to the logical extreme. A series of cuts is structured in a binary tree structure. At each node one of the input variables is considered, and a cut on this variable is found which maximises the separation between the signal and background sample. Events are then separated into left/right (yes/no) and the process is repeated, until some stopping criteria is reached. In this way the phase space is split into many regions which are classified either as signal or background like. The decision whether a region is signal or background like 
depends on which class of training events represents the majority of events in this region.

The separation gain for a cut on a variable is calculated using the so called Gini index. Using the purity of a sample consisting of $s$ signal events and $b$ background events $p=\frac{s}{s+b}$, the Gini index is $g=p \cdot(1-p)$. It is symmetric with respect to the event class, as a cut which rejects large amounts of background like events is as valuable as one which selects large amounts of signal like events.

The decision tree is considered fully grown when one of several stopping criteria is reached. If the tree reaches a depth of eight levels of cuts, the number of events in a leaf falls below 40, or no gain in separation is achieved by splitting a node further.

A model like a decision tree has many degrees of freedom. The goal of the training phase is to adjust these degrees of freedom in order to obtain a general model which can be used to label events for which the classification into signal or background is not known. Care needs to be taken not to over fit, or overtrain the decision tree. As an example consider fitting a parabola to three points. No matter where the three points lie a perfect fit is always possible, more points are required in order to make a statement about the quality of the fit and whether or not a parabola is a good generalisation of the data. In the case of a decision tree, it is possible to achieve a separation power which is too good by following fluctuations in the finite sized sample of training events. In this case the separation power achieved in training would not be achieved in an independent sample of events. A simple method, used here, to check for overtraining, is to use only half of the training sample to grow the decision tree, and use the other half to evaluate its performance once training has been completed. If the model is overtrained the performance in the second sample will be worse than in the training sample.

An individual decision tree is a weak classifier, however, a strong classifier can be constructed by combining many weak classifiers. This has the further advantage of reducing overtraining. Here we use a technique referred to as bagging. The idea is to train several decision trees on a subset of the training events, and use the average of the output of the individual classifiers 
as the combined output. Each decision tree is trained with a resampled set of events. Resampling means randomly picking a number of events from the full training sample with replacement. This procedure results in a combined classifier which is less sensitive to statistical fluctuations in the set of training events and outperforms a single decision tree.

The Boosted Decision Tree is implemented using the TMVA package, which implements several machine learning algorithms [52].

\subsection{Samples used in the training}

To train a Boosted Decision Tree at least two samples are required. A signal sample containing real electrons and a background sample containing "electrons" which are not really electrons. The second class of electrons is referred to as fake electrons. The two main sources of misidentified, or fake, electrons are: a jet containing a $\pi^{0} \rightarrow \gamma \gamma$ decay which overlaps with one of the tracks inside the jet and a photon which converts to a pair of electrons in the detector material or is associated with a random track.

In the following we describe the selection requirements both the signal and background sample. An electron is defined as a reconstructed object passing the following cuts:

1. $\mathcal{I}_{\text {cal }}<0.2$;

2. $\mathcal{F}_{\mathrm{EM}}>0.9$

3. $p_{T}>15 \mathrm{GeV}$;

4. $|\eta|<1.1$ or $1.5<|\eta|<2.5$; and

5. matched to a central track by requiring $p\left(\chi^{2}\right)_{\text {spat }}>0$.

These are referred to as preselect electrons.

\subsubsection{Real electrons}

To select real electrons from data one needs to place cuts on the variables used to separate electrons from fake electrons. However, to maximise the 
potential of the Boosted Decision Tree one wants to make these cuts as loose as possible. On the one hand if electrons with tight cuts are presented to the Boosted Decision Tree there is little room for improvement, on the other hand too loose cuts will result in objects which are not electrons being presented to the Boosted Decision Tree as electrons. Both cases would lead to a reduction in performance.

A clean sample of $\mathrm{Z} \rightarrow$ ee events is used to obtain a sample of real electrons with only minimal selection requirements applied to them. Events are required to contain two electrons, one of which has to pass tight selection requirements, while the other electron only has to fulfil the preselect electron requirements. The invariant mass of the two electrons has to satisfy: $60 \mathrm{GeV}<M_{\ell \ell}<120 \mathrm{GeV}$ (Figure 5.1). Both electrons are then used to train the Boosted Decision Tree, the tight electron is included to increase the available training statistics. For an electron to pass the tight selection requirements it has to fulfil the following requirements:

1. $\mathcal{I}_{\text {cal }}<0.07$

2. $\mathcal{F}_{\mathrm{EM}}>0.96$

3. $\chi_{\mathrm{EM}}^{2(7)}<25$;

4. $\mathcal{L}^{8}>0.8$

5. $p_{T}>17 \mathrm{GeV}$

6. $\mathrm{NN}_{7}>0.7$

7. $|\eta|<1.1$

8. matched to a track by requiring $p\left(\chi^{2}\right)_{\text {spat }}>0.001$,

9. and the electron must have fired an electron trigger.

A description of these variables can be found in Section 4.1.2. 


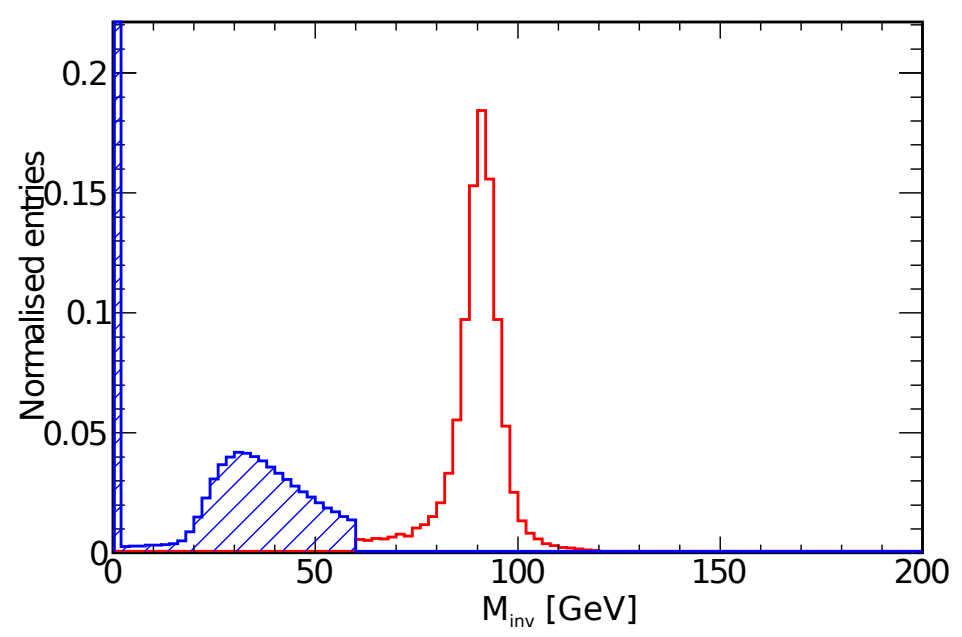

Figure 5.1: Invariant mass distribution of electrons used to train the Boosted Decision Tree. In red the distribution of electrons obtained from $\mathrm{Z} \rightarrow$ ee events, and in blue fake electrons from a sample of jets. The peak at zero arises from events where no suitable other track could be found to calculate $M_{\text {inv }}$.

\subsubsection{Fake electrons}

The majority of fake electrons arise from jets, therefore a sample consisting of events with two jets is used to obtain fake electrons to use during the training of the Boosted Decision Tree. In these events one of the jets is reconstructed as a jet, and the other as a basic electron. This type of event is selected by requiring:

1. one jet (see Section 4.1.4);

2. with a $p_{T}>15 \mathrm{GeV}$;

3. which caused a jet trigger to fire;

4. $\mathbb{E}_{T}<10 \mathrm{GeV}$; and

5. exactly one reconstructed electron. 
By limiting the amount of missing transverse energy, $\mathbb{E}_{T}$, in the event contamination from $\mathrm{W}+$ jets $\rightarrow \ell \nu+$ jets events is reduced. To further reduce the possibility of real electrons being selected the invariant mass, $M_{\mathrm{inv}}$, between the electron and any other track in the event is formed. The invariant mass $M_{\text {inv }}$ of the track-electron pair closest to $M_{Z}$ is required to be below $60 \mathrm{GeV}$ or above $120 \mathrm{GeV}$.

\subsection{Input variables and construction of the de- cision tree}

The list of potential variables offering discrimination between real and fake electrons is long. Many are considered here, including variables which are already a combination of other variables like $\mathcal{L}^{8}$. While decision trees are robust against the inclusion of variables which offer little separation power, the minimal set needed to achieve the same performance are included. Input variables can be separated into three groups: variables defined in the central calorimeter, in the endcap calorimeter, and variables related to the tracking detectors. The distributions of the input variables defined in the central and endcap calorimeter are shown in Appendix B.1 and Appendix B.2, respectively.

Four separate Boosted Decision Trees are constructed, corresponding to the four combinations: central and endcap calorimeter, high and low instantaneous luminosity. The split into two disjoint regions corresponding to the two calorimeter regions is motivated by the different signal to background ratios, and the availability of different variables in the two regions. The signal to background ratios, as well as the resolution of variables vary between high and low instantaneous luminosity, and as a result separate Boosted Decision Trees are trained. This division into four categories improves the overall performance by reducing the correlation between variables, and separating regions of different signal to background ratios. For example, a variable might offer better separation power at lower than at higher instantaneous luminosity, or a variables separation power might depend on the $\eta$ of the 
Table 5.1: Variables used for low instantaneous luminosity events in the central and endcap Boosted Decision Trees.

\begin{tabular}{cc}
\hline Central calorimeter & Endcap calorimter \\
\hline $\mathcal{L}^{8}$ & $\mathcal{L}^{8}$ \\
$\mathrm{NN}_{7}$ & $\mathrm{NN}_{4}^{\gamma}$ \\
$\mathcal{I}_{\text {cal }}$ & $\mathrm{NN}_{3}$ \\
cps_sq_rms & $\mathcal{I}_{\text {cal }}$ \\
cps_rms & prbtrk \\
prbtrk_eop & prbtrk_eop \\
prbtrk & $\frac{E_{\text {cal }}}{p_{\text {trk }}}$ \\
$\frac{E_{\text {cal }}}{p_{\text {trk }}}$ & $\#$ layer0 hits \\
$\#$ tracks in a $\Delta R=0.05$ & $\#$ tracks in a $\Delta R=0.05$ \\
Hits On The Road & $\#$ smt hits \\
\hline
\end{tabular}

electron. In both cases performance is increased by training Boosted Decision Trees separately for each region. More details and examples can be found in Reference [52].

Each Boosted Decision Tree consists of 200 individual decision trees which are trained with a resampled set of events. The Boosted Decision Tree for high luminosity events, in the endcap region, suffers from overtraining as this is the region with the lowest number of training events. To reduce the number of degrees of freedom this Boosted Decision Tree consists of only 100 trees. Tables 5.1 and 5.2 list the ten input variables used for each of the four Boosted Decision Trees.

\subsection{Performance of the BDT}

During the training of the Boosted Decision Tree only half of the available signal and background electrons are used, this is the training set. The other half is the testing set. After training the Boosted Decision Trees the output distributions for signal and background like electrons in the training and testing set are compared. If there is no overtraining the two distributions will agree. In Figures 5.2 and 5.3 the output distributions of the Boosted Decision Trees for the central and endcap regions of the calorimeter are shown. 
Table 5.2: Variables used for high instantaneous luminosity events in the central and endcap calorimter Boosted Decision Trees.

\begin{tabular}{cc}
\hline Central calorimeter & Endcap calorimeter \\
\hline $\mathcal{L}^{8}$ & $\mathcal{L}^{8}$ \\
$\mathrm{NN}_{7}$ & prbtrk $^{\circ}$ \\
cps_sq_rms & $\mathrm{NN}_{4}^{\gamma}$ \\
prbtrk_eop & prbtrk_eop \\
cps_rms & $\mathrm{NN}_{3}$ \\
$\overline{\mathcal{I}}_{\text {cal }}$ & $\frac{E}{p_{T}}$ \\
prbtrk & $\mathcal{I}_{\text {cal }}$ \\
$\mathcal{F}_{\text {EM }}$ & $\#$ tracks in a $\Delta R=0.05$ \\
$\#$ tracks in a $\Delta R=0.05$ & $\#$ layer0 hits \\
\hline
\end{tabular}

Signal like electrons accumulate at +1 and background like electrons at -1 . Furthermore the shape of the Boosted Decision Tree output distributions agree well between training and testing samples.

The Boosted Decision Tree output is a continuos variable, therefore it is possible to place a cut on this variable at any value. Electrons with a Boosted Decision Tree output above the cut are kept, and treated as real electrons, whereas electrons below the cut are rejected. Cutting looser (closer to -1) will increase the efficiency for real electrons but also increase he rate at which fake electrons are mistakenly classified as electrons. The relation between the efficiency and the fake rate is shown in Figure 5.4. Naturally, the aim is to achieve as low a fake rate as possible for as high a signal efficiency as possible. The efficiency and fake rate for a selection of the traditional electron identification criteria, based on cuts alone, are shown as well. In comparison to these points the Boosted Decision Tree based electron identification achieves the same efficiency at a lower fake rate, or a higher efficiency at the same fake rate. For a given efficiency (or fake rate) one can read off the corresponding cut value from Figures 5.5 and 5.6 for central and endcap calorimeter electrons respectively. Table 5.3 lists the cut values which give comparable performance to the traditional electron identification criteria in either efficiency or fake rate.

In addition to the integrated efficiency and fake rate, the dependency 


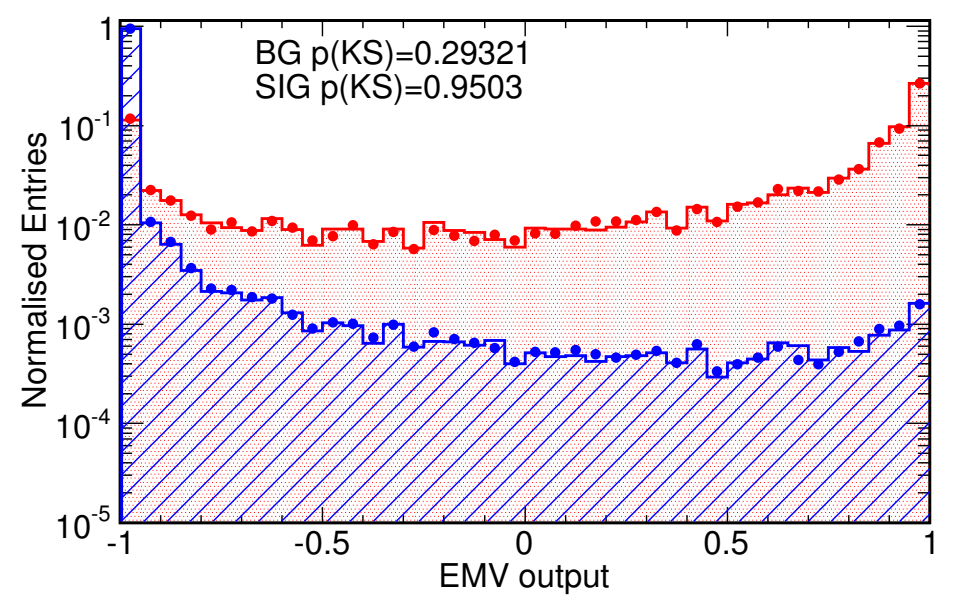

(a)

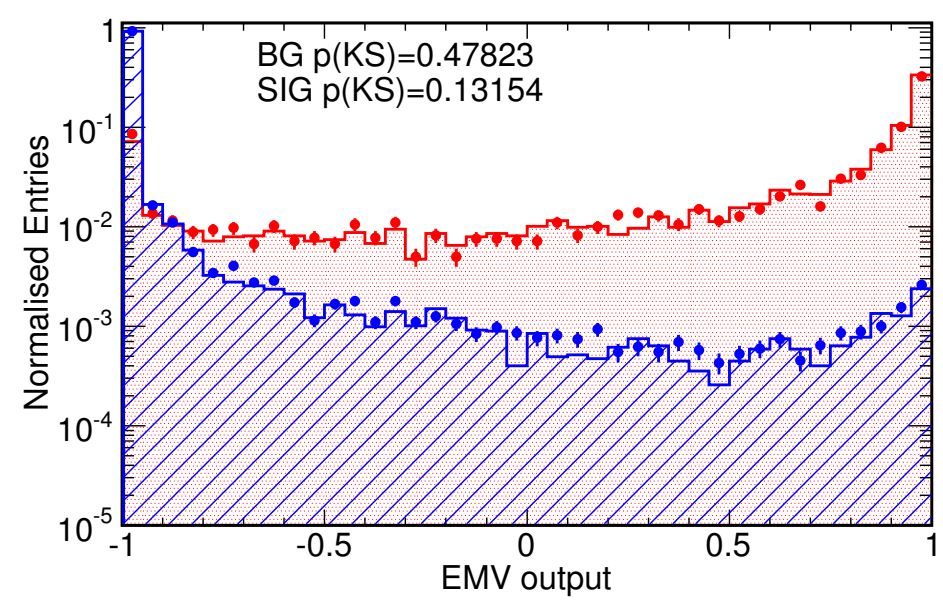

(b)

Figure 5.2: Boosted Decision Tree output for electrons in the central calorimeter. The Boosted Decision Tree for (a) low luminosity events and (b) high luminosity events. In red the distribution of real electrons, compared to the distributions of fake electrons in blue. The histogram shows the output distribution obtained from the training dataset and the points show the testing dataset. 


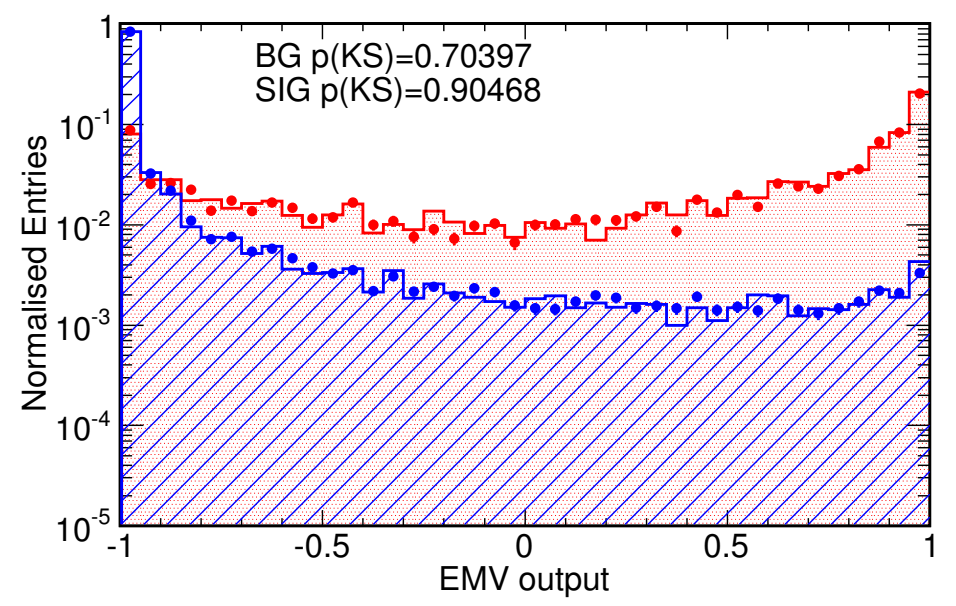

(a)

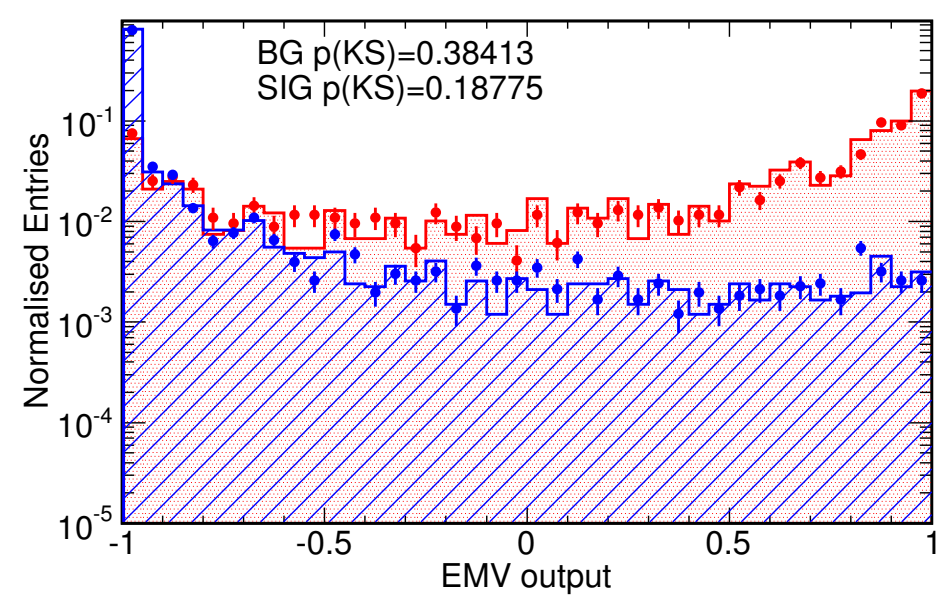

(b)

Figure 5.3: Boosted Decision Tree output for electrons in the endcap calorimeters. The Boosted Decision Tree for (a) low luminosity events and (b) high luminosity events. In red the distribution of real electrons, compared to the distributions of fake electrons in blue. The histogram shows the output distribution obtained from the training dataset and the points show the testing dataset. 


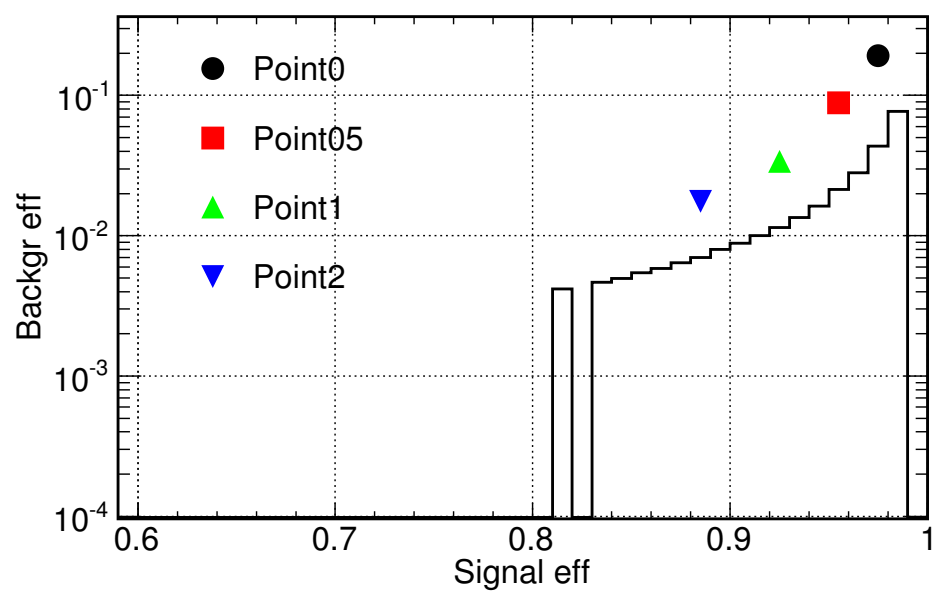

(a)

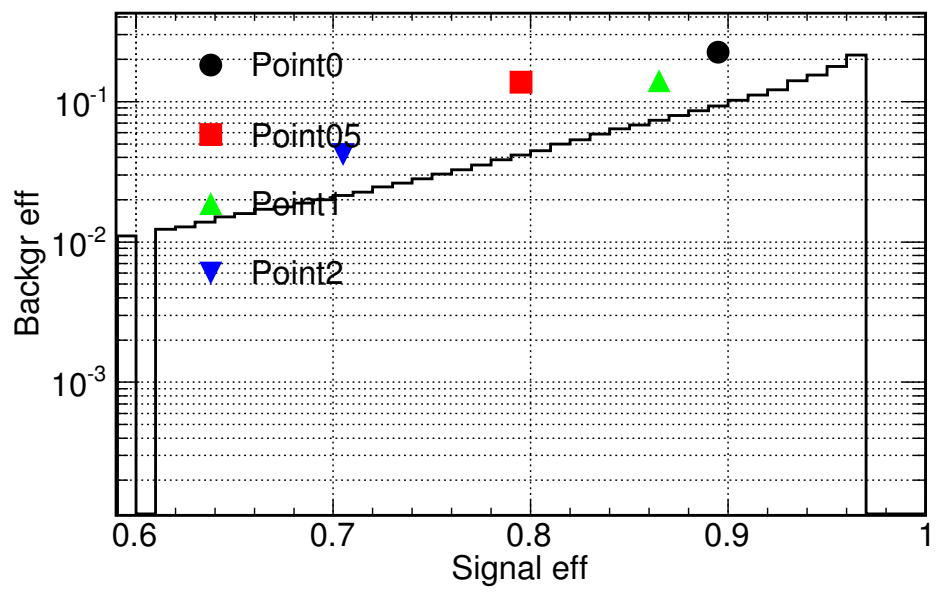

(b)

Figure 5.4: The solid black line in this figure shows the dependence of the fake rate on the efficiency separately for (a) the central and (b) the endcap calorimeter region. For comparison the current cut based electron identification points are shown. Ideally one wants to achieve as low a fake rate as possible for as high a signal efficiency as possible. 


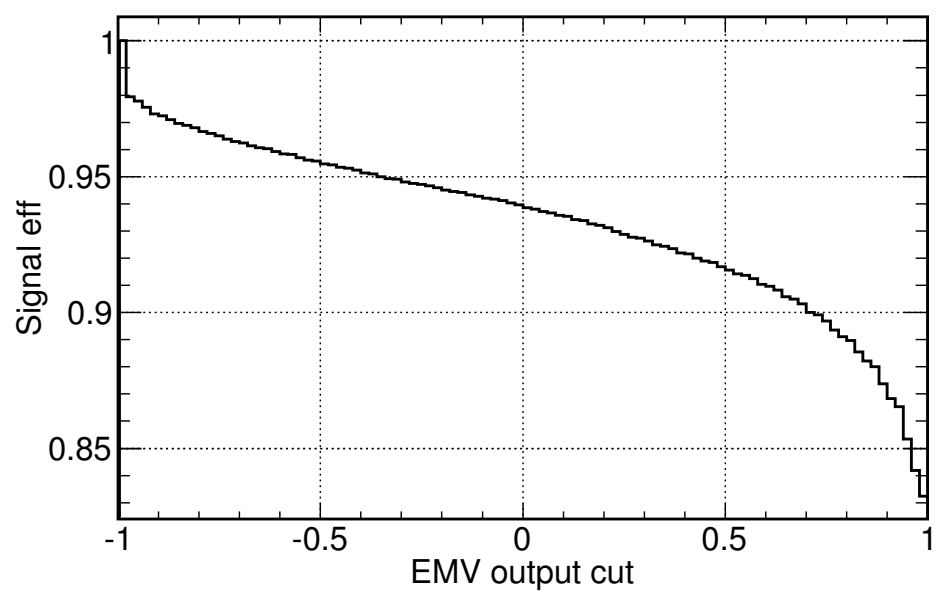

(a)

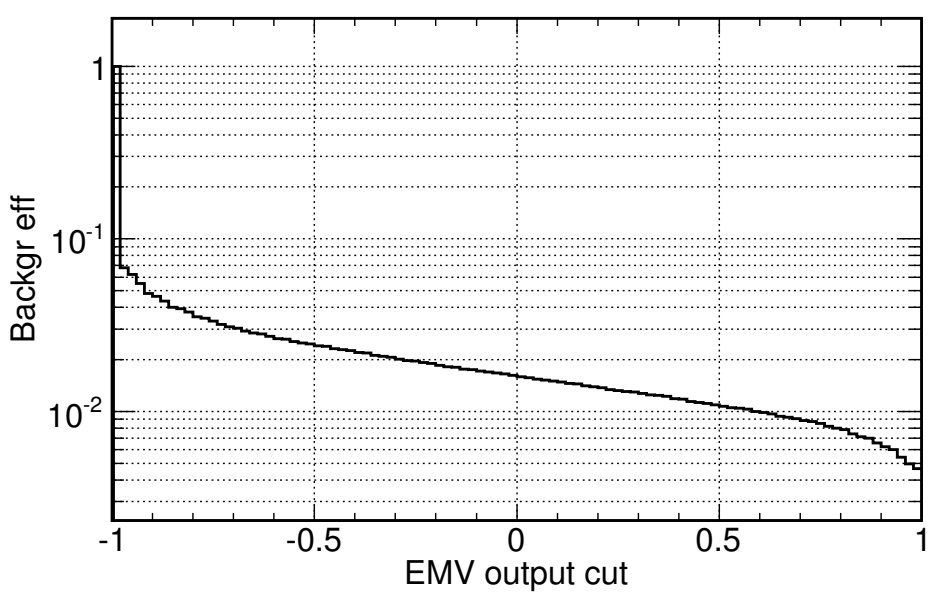

(b)

Figure 5.5: As the cut on the Boosted Decision Tree output becomes tighter (a) the efficiency for real electrons reduces. The fake rate as a function of the cut value is shown in (b). These plots are for central calorimeter electrons. 


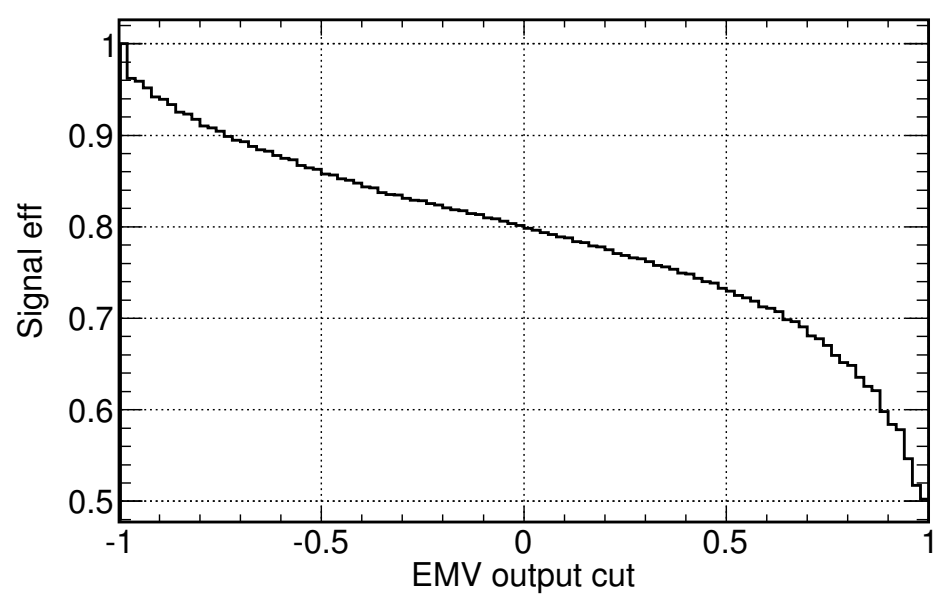

(a)

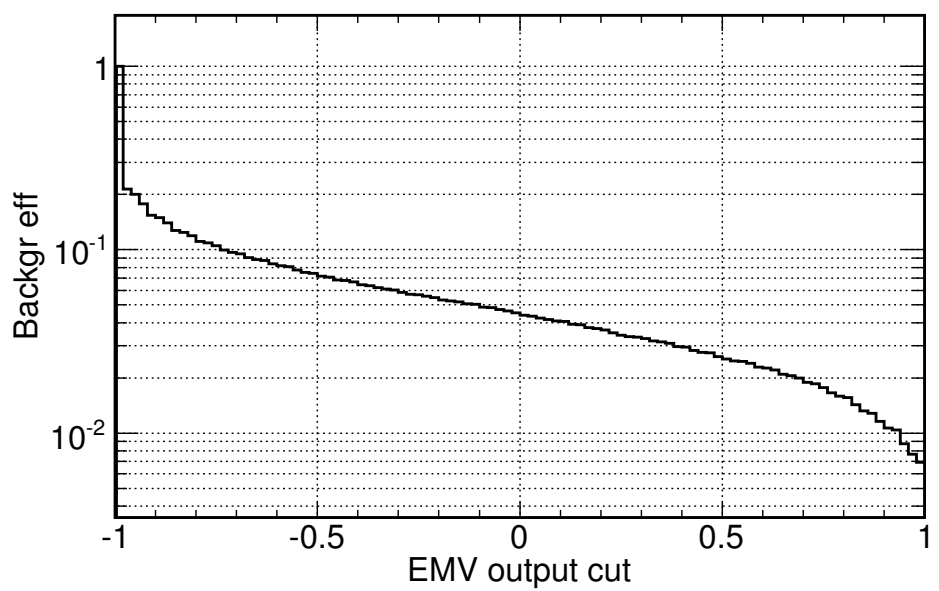

(b)

Figure 5.6: As the cut on the Boosted Decision Tree output becomes tighter (a) the efficiency for real electrons reduces. The fake rate as a function of the cut value is shown in (b). These plots are for endcap calorimeter electrons. 
Table 5.3: Cut values to achieve a fake rate similar to the current electron identification points. The cut value for Point1€ achieves similar efficiency, while Point1 fake achieves similar fake rate.

\begin{tabular}{lccccc}
\hline & Point05€ & Point1 $€$ & Point2 $€$ & Point1 fake & Point2 fake \\
\hline Central & -0.96 & -0.22 & 0.36 & -0.86 & -0.32 \\
Endcap & -0.86 & -0.74 & 0.38 & -0.86 & -0.02 \\
\hline
\end{tabular}

of the efficiency on the $p_{T}$ and $\eta$ of the electron, as well as the instantaneous luminosity give information about how the Boosted Decision Tree based electron identification performs. For example, it could be possible that the increase in efficiency is only present for very large values of electron $p_{T}$, while at typical values of electron $p_{T}$ it performs no better than the traditional electron identification.

In Figures 5.7 and 5.8 the efficiency and fake rate are shown for electrons in the central and endcap calorimeter regions. The cut on the Boosted Decision Tree was chosen so that the integrated efficiency matched that of the Point1 cut based electron identification. 

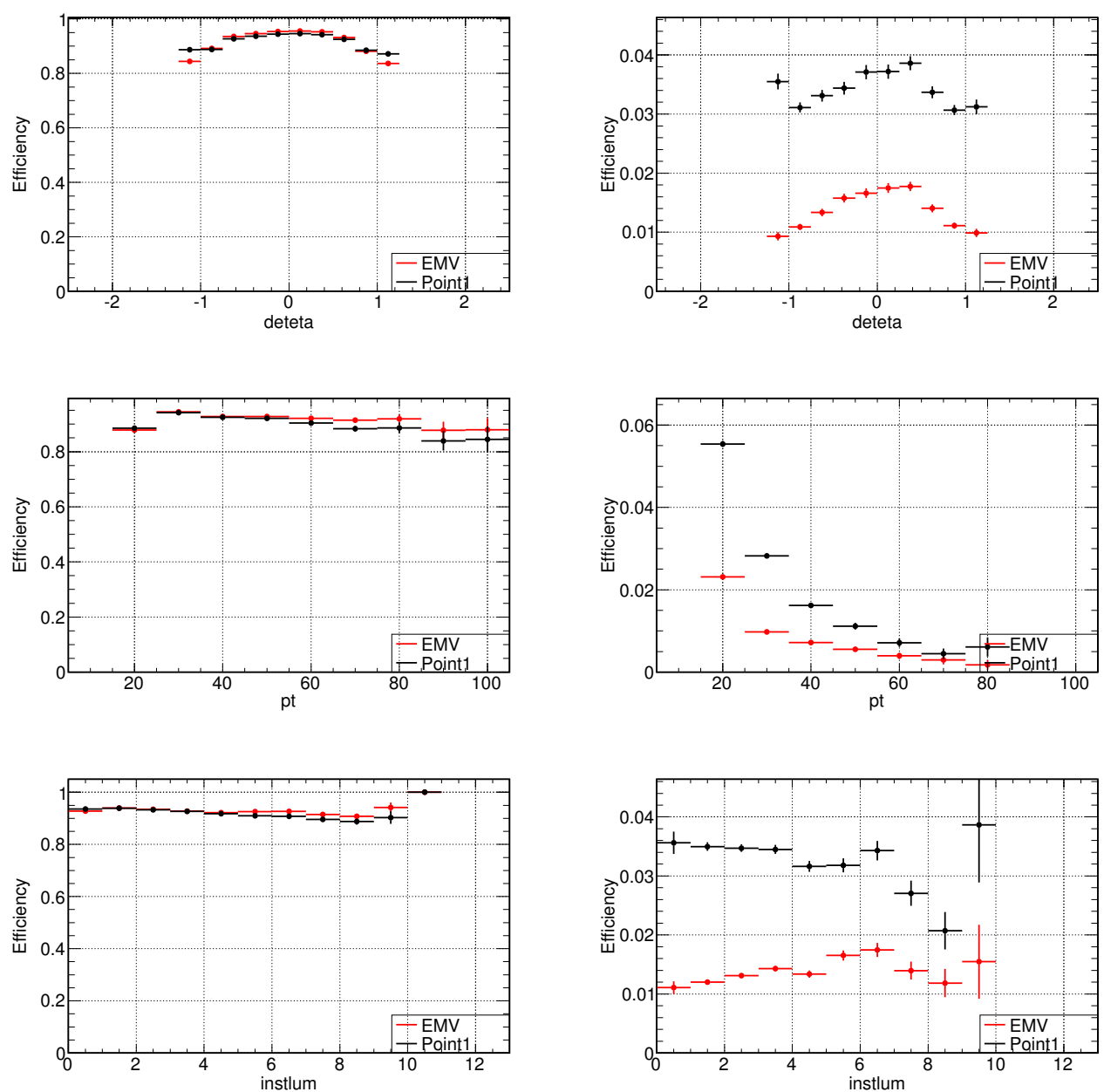

Figure 5.7: Dependency of the efficiency (left) and fake rate (right), for electrons in the central calorimeter. The Boosted Decision Tree cut was tuned to match the efficiency of the cut based Point1. In the left hand column the efficiency for real electrons as a function of detector $\eta, p_{T}$ and instantaneous luminosity is shown. The right hand column shows the fake rate for the same three variables. Plotted in red is the dependency of the Boosted Decision Tree and in black that of Point1. 

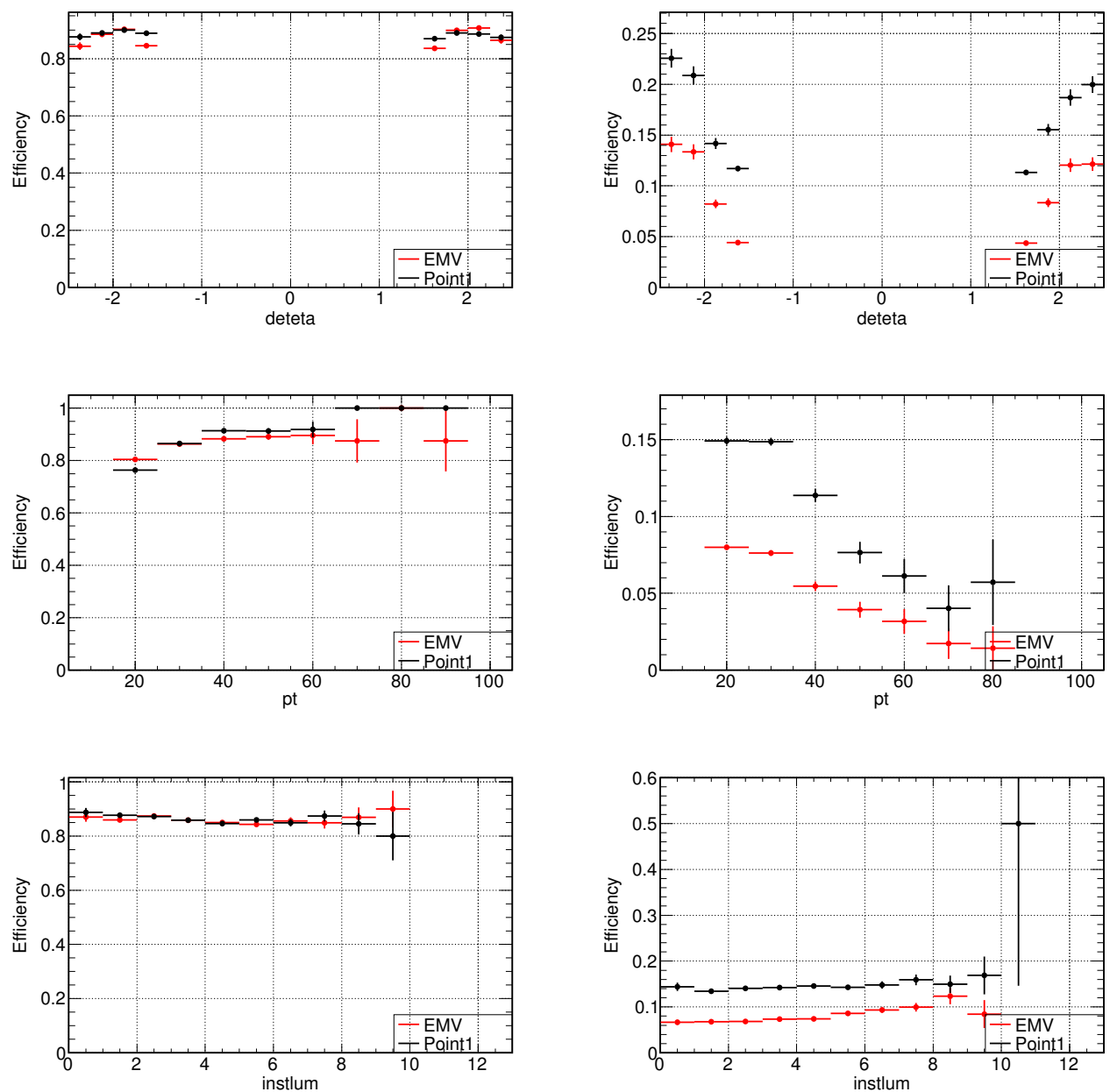

Figure 5.8: Dependency of the efficiency (left) and fake rate (right), for electrons in the endcap calorimeter. The Boosted Decision Tree cut was tuned to match the efficiency of the cut based Point1. In the left hand column the efficiency for real electrons as a function of detector $\eta, p_{T}$ and instantaneous luminosity is shown. The right hand column shows the fake rate for the same three variables. Plotted in red is the dependency of the Boosted Decision Tree and in black that of Point1. 


\section{Chapter 6}

\section{Event selection}

This section presents the selection criteria used to extract a high purity sample of $t \bar{t}$ events from the data. The aim is to select $t \bar{t}$ signal events with as large an efficiency as possible while rejecting background processes with much larger production cross-sections. The same selection is used for all analyses presented in this thesis.

The topology of the dilepton final state is characterised by two leptons of opposite charge, two jets originating from $b$-jets and an imbalance in transverse energy arising from two neutrinos. The main physics background arises from $Z \rightarrow \ell^{-} \ell^{+}+$jets events. The event selection takes advantage of the kinematic and topological differences between the signal and background processes. It is based on the dilepton cross section measurement detailed in Reference [11].

In the two analyses described in this thesis data from two distinct data taking periods is used. The first period lasted from April 2002 to February 2006 corresponds to an integrated luminosity of about $1.1 \mathrm{fb}^{-1}$. It will be referred to as Run IIa. Following an upgrade of the D0 detector data taking resumed in June 2006. The analyses in this theses make use of data taken up June 2009. This period is referred to as Run IIb. The integrated luminosity of Run-IIb is $4.3 \mathrm{fb}^{-1}$. The combined dataset corresponds to an integrated luminosity of $5.4 \mathrm{fb}^{-1}$. Data from these two periods is analysed separately as data taking conditions are different between the two, for example the average 
instantaneous luminosity in Run IIa is much lower than during Run IIb.

The dilepton final state can be split into three channels according to the flavour of the charged leptons. Decays of tét events containing two electrons (dielectron channel), one electron and one muon (e $\mu$ channel) or two muons (dimuon channel) are considered separately. The selection efficiency and background rejection rate depends on the flavour of the leptons, therefore the selection criteria are optimised for each channel individually. Across all three channels the following selection criteria are shared:

- Periods during which the quality of data taking is considered poor are removed by rejecting luminosity blocks marked as bad;

- the primary vertex must have at least three tracks associated with it and satisfy $|z|<60 \mathrm{~cm}$;

- two oppositely charged leptons, with $p_{T}>15 \mathrm{GeV}$. Electrons must be within $|\eta|<1.1$ or $1.5<|\eta|<2.5$, and muons must satisfy $|\eta|<2$; and

- at least two jets with $p_{T}>20 \mathrm{GeV}$ and $|\eta|<2.5$. For data taken during Run IIb jets must contain at least two tracks associated with the primary vertex.

They are motivated by the topology of the final state: two jets originating from the quark into which the top quark decays and one charged lepton each from the decay of the two $\mathrm{W}$ bosons. The pseudo-rapidity requirements are dictated by the geometry of the detector. At this stage the dimuon and dielectron channels are dominated by $Z+$ jets events. This provides an opportunity to validate the modelling of the main background by the simulation in a high statistics sample. This stage of the selection is referred to as the basic selection.

The e $\mu$ channel has the best signal to background ratio of the three final states. As the physics backgrounds are small and the branching ratio is twice as large as for the dielectron or dimuon channel. The main physics background arises from $\mathrm{Z} \rightarrow \tau \tau$ production where one $\tau$ lepton decays to 
an electron and one $\tau$ lepton decays to a muon. The decay of the $\tau$ lepton includes two undetected neutrinos:

$$
\tau \rightarrow W v_{\tau} \rightarrow \ell v_{\ell} v_{\tau}
$$

where $\ell=e, \mu$. As a result the leptons which are detected only carry part of the momentum of the $\tau$ lepton, leading to the $p_{T}$ distribution of these leptons being softer than for leptons from te decays. Furthermore, jets in $\mathrm{Z} \rightarrow \ell \ell$ production arise mainly from soft radiation. As a result the transverse

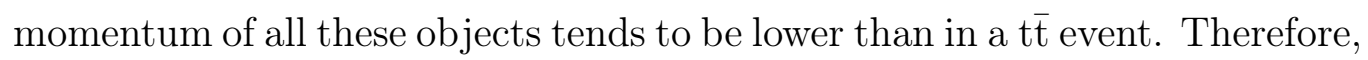
the scalar sum of transverse momenta of the jets and highest transverse momentum lepton, $H_{T}$, is used to discriminate against this background. In the e $\mu$ channel the only requirement beyond the basic selection is that events have to satisfy $H_{T}>110 \mathrm{GeV}$.

In the dimuon and dielectron channels the dominant process after the basic selection requirements is $\mathrm{Z} \rightarrow \ell \ell$ production. While no imbalance in the missing transverse energy, $\mathbb{E}_{T}$, is expected in this process, mismeasurement of the energies of the visible objects gives rise to an apparent imbalance. In $t \bar{t}$ signal events the presence of two neutrinos gives rise to a real imbalance in the missing transverse energy. To discriminate between the two cases events are required to have a significant amount of missing transverse energy $\sigma_{\mathbb{F}_{T}}$. The resolution of the missing transverse energy is determined by many effects including: wrong choice of the primary vertex, mismeasurement of lepton $p_{T}$ or energy resolution of jets. For a given event, with some measured $E_{T}$ we would like to know whether this is likely due to a mismeasurement or not. The $\sigma_{E_{T}}$ variable takes into account the particular topology and physics objects present in an event. The resolution of all physics objects are found to be well described by a gaussian distribution, the $\mathbb{E}_{T}$ distribution is therefore modelled as a gaussian distribution as well. Finally, $\sigma_{\mathbb{F}_{T}}$ is the pseudo likelihood defined by:

$$
\sigma_{\mathscr{E}_{T}}=\log \frac{p\left(\mathbb{E}_{T}\right)}{p(0)}
$$


where $p(x)$ is a gaussian probability density with mean $\mathbb{E}_{T}$ and width given by the sum of the resolution of the individual physics objects along the direction of the measured $\mathbb{E}_{T}$. An in detail description of the algorithm and parametrisation of the resolutions is given in Reference [68].

In the dimuon and dielectron channels we require $\sigma_{\mathbb{E}_{T}}>5$. Additionally a large amount of missing transverse energy is required in the dimuon channel: $E_{T}>40 \mathrm{GeV}$.

A further source of background events in all three channels is the production of pairs of vector bosons, WW, WZ and ZZ, collectively referred to as diboson events. In diboson production jets arise mainly from soft radiation, combined with the fact that their production cross section is orders of magnitude smaller than for $Z+$ jets production no further cuts are needed to achieve good rejection of this background.

The main instrumental background arises from the misidentification of jets as muons in events with one leptonic $\mathrm{W}$ boson decay and at least three jets, one of which is misidentified as a charged lepton. Semileptonic decays of $c$ or $b$ hadrons inside of jets can produce a lepton. While these are real leptons, in the context of these analyses they are considered as a misidentified lepton as they do not originate from the hard scattering process. In addition to requiring charged leptons to be spatially separated from jets and that the pair of leptons is of opposite charge, lepton identification quality criteria are used to reduce the probability of misidentifying leptons. However the cross section for $W+$ jets production is large resulting in a non negligible number of events with misidentified muons. Jets can also be misidentified as an electron if they contain a $\pi \rightarrow \gamma \gamma$ decay, which overlaps with a random track in the jet. The large amount of electromagnetic energy deposited in the calorimeter, combined with the track mimics the signature of a real electron.

Events in the e $\mu$ channel are accepted regardless of which specific trigger caused the event to be recorded. In the dielectron channel events recorded by any trigger requiring a single electron are used. These triggers require an energy deposit in the EM section of the calorimeter. The logical OR of all these triggers was measured to be nearly $100 \%$ efficient $[67,53]$. Similarly, in the dimuon channel events recorded by any of the triggers requiring a single 
muon are considered.

A comparison between the simulation and the data for the three main variables used to discriminate against the background events at the stage of the basic selection are shown in Figure 6.1. A full set of plots comparing data and simulation at the stage of the basic and final selections are shown in Appendix A. The predicted number of events, separated by process, are compared to the data in Tables 6.1 and 6.2. Yields are compared after the basic selection, after applying the final selection requirements and after requiring that the four momenta of the top quark and antiquark could be reconstructed. More details of this reconstruction will be given in Section 7.2.

The data samples, once selected, are compared to Monte Carlo simulations of each of the contributing processes. Except for the background contribution arising from misidentification of jets as charged leptons, which is estimated purely from data.

The t $\bar{t}$ signal events are generated using the MC@NLO 3.4 event generator, interfaced to HeRwIG for parton showering and fragmentation. The MC@NLO event generator allows the generation of samples with and without tĖ spin correlations. When neglecting spin correlations MC@NLO generates the hard scattering event, including the top quark and antiquark, however it hands these to HERWIG which in this case simulates the decay of the t $\bar{t}$ pair. No information about the spins is passed to HeRwiG, as a result the decay proceeds as if the top quark and antiquark became decorrelated. To generate samples including SM spin correlations, MC@NLO performs the decay of the top quark and antiquark itself. As it has full access to the spin information the angular distributions of the decay products reflect the spin orientation of their parent particle. Events are weighted to reproduce the calculated approximate-NNLO cross section of $7.46 \mathrm{pb}$ [64].

The diboson backgrounds are simulated using PYTHIA, both samples are normalised to the NLO cross section calculated by MCFM [36], which is about $40 \%$ larger than the leading order cross section used in PYTHIA.

$Z+$ jets events are simulated using Alpgen, followed by PYTHIA for showering and hadronisation. In this setup the hard scatter is simulated using matrix elements in ALPGEN. PYTHIA then performs the evolution of 


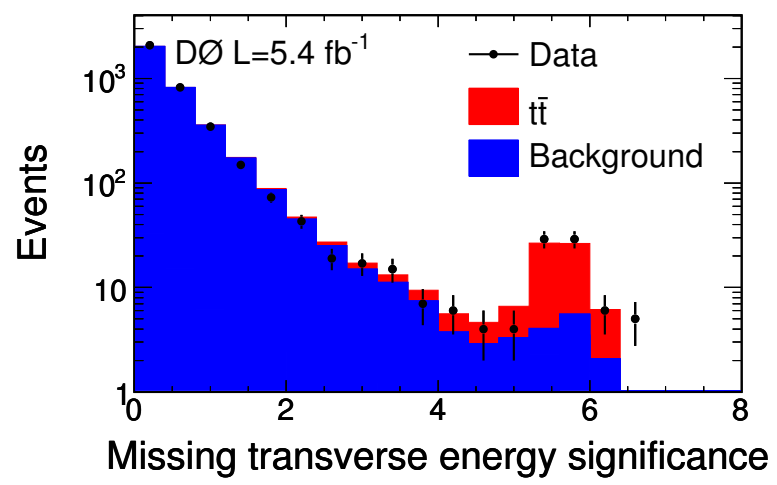

(a)

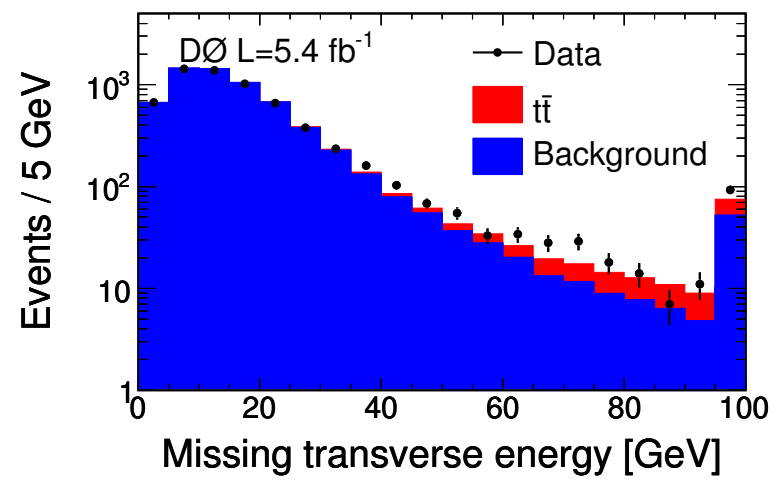

(b)

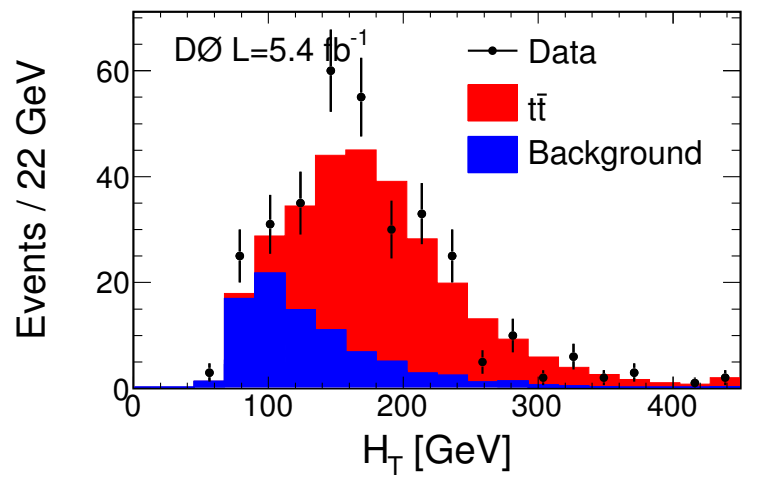

(c)

Figure 6.1: Three variables used to separate $t \bar{t}$ signal events from the background after the basic selection. The $\sigma_{E_{T}}$ distribution in the dielectron channel is shown in (a), in (b) the $\mathbb{E}_{T}$ distribution in the dimuon channel is shown in the middle and in (c) the $H_{T}$ distribution in the $\mathrm{e} \mu$ final state. 
Table 6.1: Expected event yields for each channel in Run IIa. The total

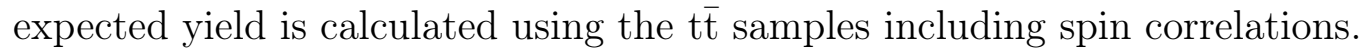
The final line in each channel gives the yields after requiring that the event kinematics could be solved for in addition to the final selection requirements.

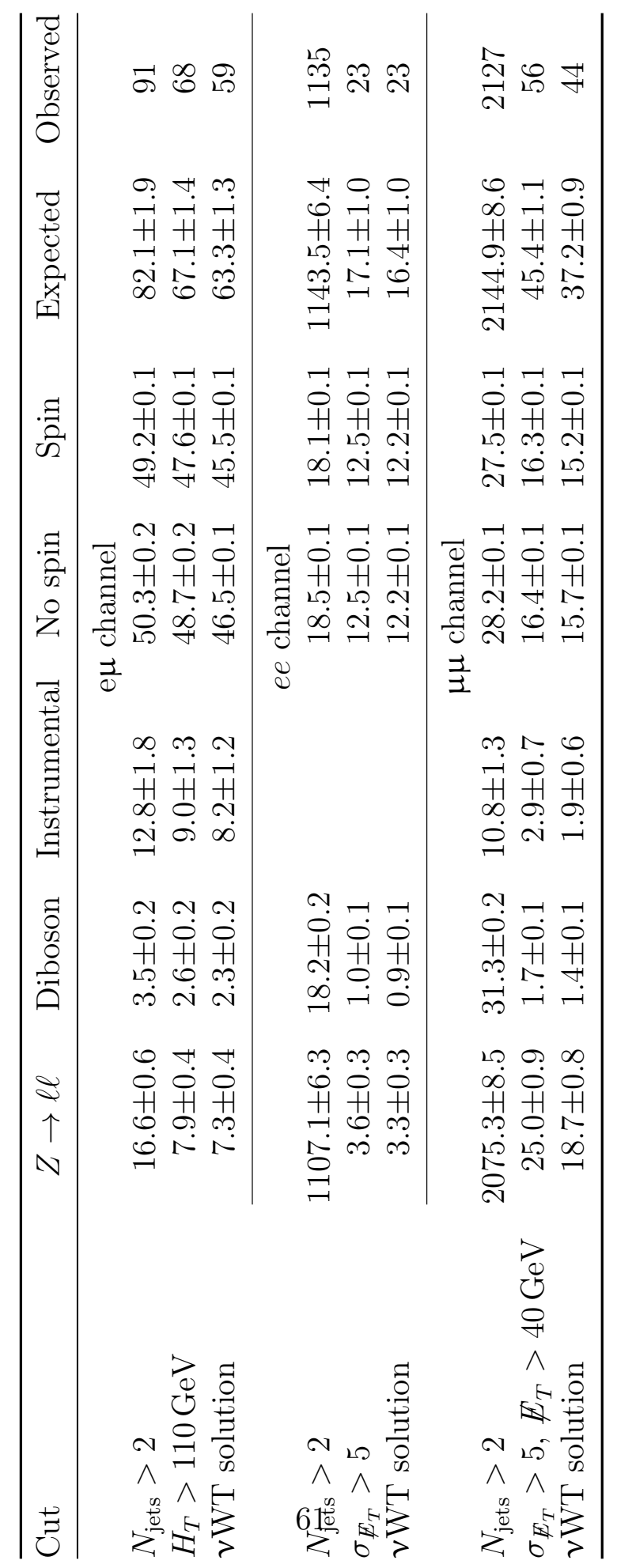


Table 6.2: Expected event yields for each channel in Run IIb. The total

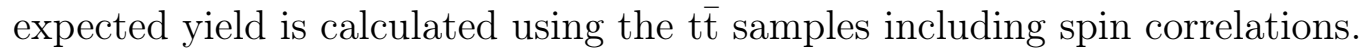
The final line in each channel gives the yields after requiring that the event kinematics could be solved for in addition to the final selection requirements.

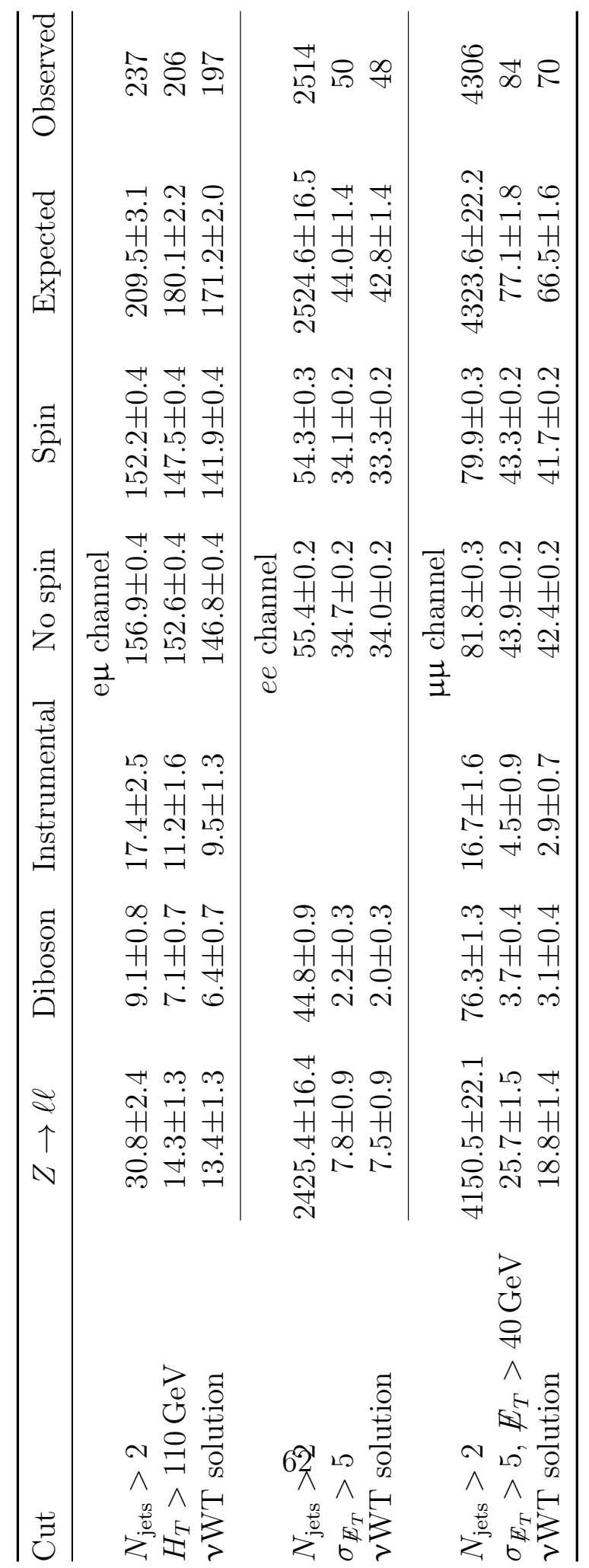


the parton shower and hadronisation of the partons. As a result there is the possibility for overlap between extra partons generated by the matrix element generator and partons generated by the showering. To remove this double counting the MLM matching scheme is applied [24]. Events are weighted to achieve a normalisation according to the NNLO cross section. The distribution of the transverse momentum of the $\mathrm{Z}$ boson indirectly determines the jet multiplicity of $Z+$ jets events. The $\mathrm{Z}$ boson $p_{T}$ distribution in data is not well modelled by the simulation and a reweighting derived from data is applied to improve the description.

The two sources of fake electrons produce electrons which barely pass the isolation requirements and have a low value for the electron likelihood $\mathcal{L}^{8}$, where as real electrons have a value close to one. The number of fake electrons is evaluated by fitting two templates to the $\mathcal{L}^{8}$ distribution after all selection cuts. The shape of the first template represents that of fake electrons and the second the shape of the distribution for real electrons. The two templates are shown in Figure 6.2. The template for fake electrons is taken from a sample of multijet events in data, and the template for real electrons from a sample of pure $Z \rightarrow e e$ events [30]. The instrumental background arising from jets passing the electron identification criteria is evaluated as being negligible.

A further instrumental background are muons which appear isolated but originate from within jets. Events of this type which pass the event selection mainly contain one isolated electron/muon and one fake isolated muon from heavy flavour quark decays. The number of fake isolated muons is evaluated by multiplying the fake muon isolation probability, the rate at which a nonisolated muon appears to be isolated, with the number of events passing the normal selection cuts containing two isolated leptons of the same charge. The fake muon isolation probability is evaluated in a sample of $b \bar{b}$ events. This sample is selected by requiring two muons. One of which is not isolated and within $(\Delta \mathcal{R}<0.5)$ a jet and no isolation requirements are made for the second muon. The rate at which a non-isolated muon in these events also passes the isolated muon requirements is used as the fake muon isolation probability. In events with two jets it is found to be $16.1 \pm 1.17 \%$.

The data are compared to simulation at two different stages of the se- 


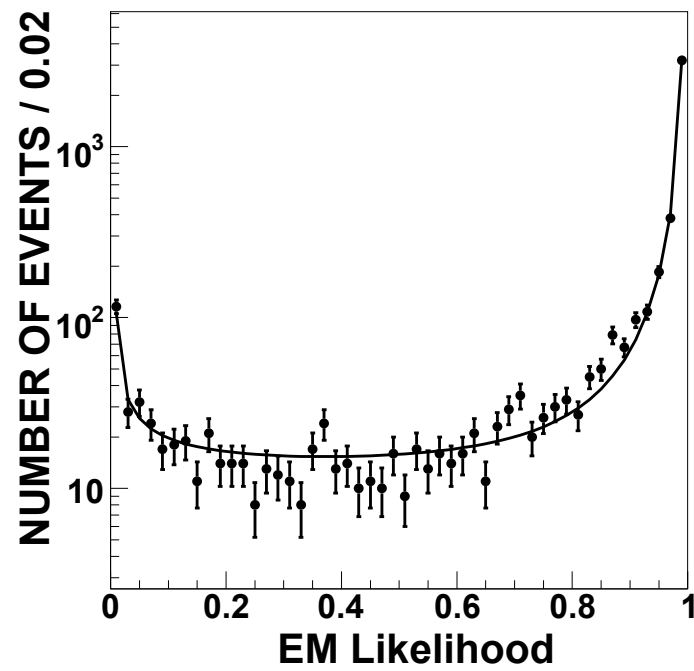

(a)

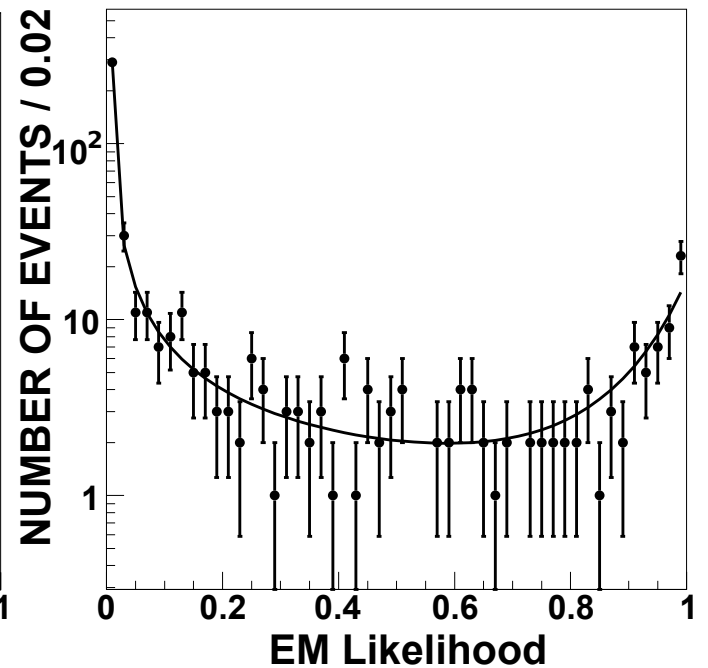

(b)

Figure 6.2: Electron likelihood, $\mathcal{L}^{8}$, distributions for (a) real electrons from $Z \rightarrow e e$ data and (b) fake electrons from a multijet and $W+$ jets dominated sample [30].

lection. After applying a basic selection of requiring two oppositely charged leptons and two jets most events are predicted to originate from $Z+$ jets events. This provides a high statistics sample to validate the modelling of the data by the simulation. At the same time the selection requirements are very similar to the final ones used in the analysis.

A deficit of events is found when applying the basic selection criteria only. This is attributed to a persisting mismodelling of the $\mathrm{Z}$ boson $p_{T}$ distribution. An ad-hoc scaling of the $Z+$ jets contribution is derived by dividing the number of events in data by the number of events predicted by the $\mathrm{MC}$ for events close to the $\mathrm{Z}$ boson mass peak $\left(80<m_{\ell \ell}<100 \mathrm{GeV}\right)$. In Figure 6.3 the invariant mass distributions are shown before and after the scaling. Both analyses use this scaling of the $Z+$ jets background in the dimuon and dielectron samples. 


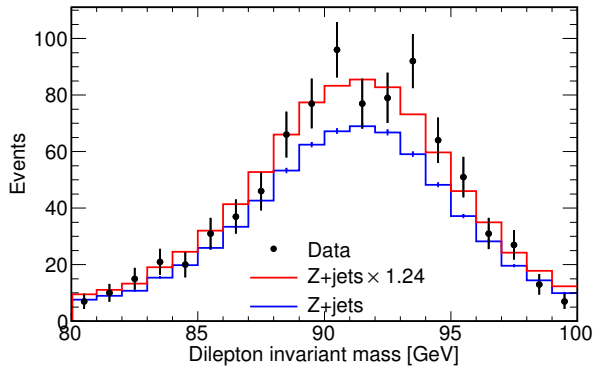

(a)

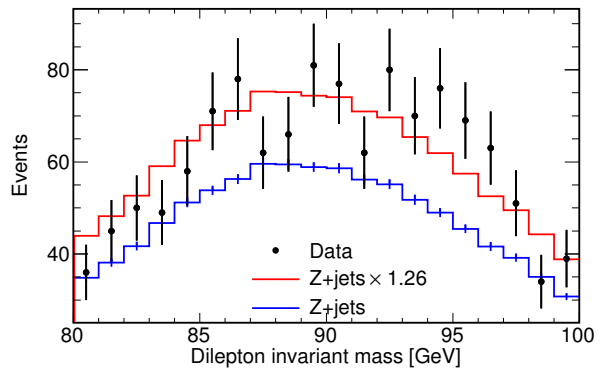

(c)

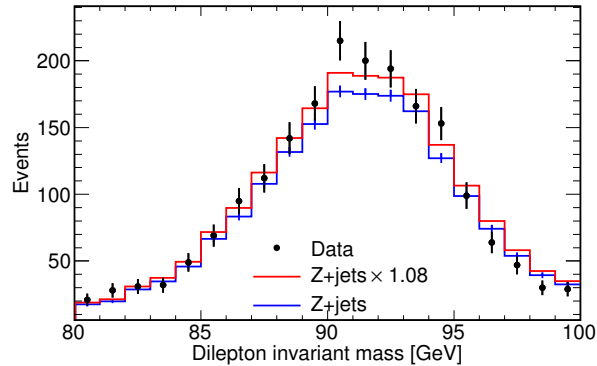

(b)

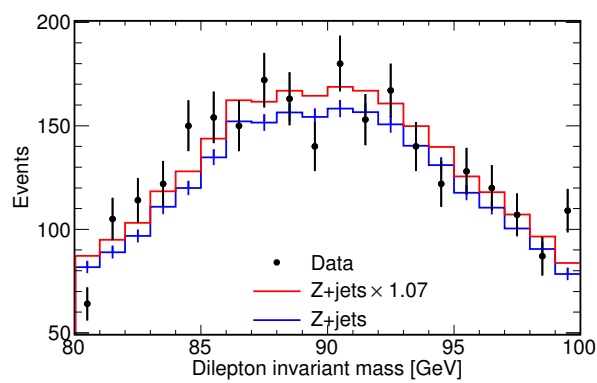

(d)

Figure 6.3: Plots showing the dilepton invariant mass distributions close to the $\mathrm{Z}$ boson mass peak. The Run IIa and Run IIb dielectron invariant mass distribution is shown in (a) and (b). In (c) and (d) the dimuon invariant mass distribution is shown. The default $\mathrm{Z}+$ jets $\mathrm{MC}$ is shown in blue and in red it is scaled to have the same yield as the data. 


\section{Chapter 7}

\section{Spin Correlation}

One of the basic properties of the top quark, its spin, is still basically unexplored. In the SM a significant correlation is expected between the direction of the spin of the top quark and anti-quark. The strength of the correlation depends on the production mechanism, for $q \bar{q}$ production a strong correlation is expected, where as for $t \bar{t}$ pairs produced via $g g$ fusion the correlation is expected to be small $[61,62]$. The top quark decays through the electroweak interaction before it can form bound states $[9,31]$. The angular distributions of the final state particles reflect the spin orientation of the top quark at production [27]. Using a joint angular distribution of a top quark and antiquark decay product the degree to which the direction of the spins of the top quark and anti-quark are correlated can be measured.

Heavy quark pair production via QCD processes results in a significant spin correlation. However, for bottom and charm production these effects are not observable as they form bound states and hadronise before decaying. Whereas the weak decay of the top quark takes place before toponium like bound states can be formed.

The main production mechanism for top pairs at the Tevatron is $q \bar{q} \rightarrow t \bar{t}$ which proceeds via the exchange of one gluon. As a result the $t \bar{t}$ pair is produced in a ${ }^{3} \mathrm{~S}_{1}$ state, in which the $\mathrm{t}$ and $\overline{\mathrm{t}}$ spins are both either aligned parallel or antiparallel to the momentum of the incoming quark. This leads to the charged leptons from $t$ and $\bar{t}$ being preferentially emitted in opposite 
hemispheres. If the spins were uncorrelated or became decorrelated before the decay the leptons would be emitted uniformly into either hemisphere. The gluon fusion process produces t $\overline{\mathrm{t}}$ pairs in a ${ }^{1} \mathrm{~S}_{0}$ state, where the spins of the top quarks are antiparallel.

This chapter starts with an explanation of how to construct an observable sensitive to spin correlations, followed by a description of the algorithm used to fully reconstruct the kinematics of each event. The third section details the method used to extract the amount of spin correlation from the data and how to incorporate systematic uncertainties into the measurement process. The process of setting limits on a physical parameter which has a narrow range of allowed values is discussed in Section 7.4. As the basic measurement is limited by the available statistics, an improved measurement employing a method based on the matrix element technique is described in Section 7.5. The second to last sections outlines the sources of systematic uncertainties considered. The final results are given in the final section. Comparisons to more recent results from other experiments are made. To begin the journey we must first understand what it is we want to measure.

\subsection{Observable}

Constructing an observable for top quark spin correlations requires understanding of both the production and decay mechanism of the top quark. The overall observable is introduced first, followed by a discussion of the relevant production and then decay properties.

An observable sensitive to spin correlation of a top quark pair, can be constructed by combining an observable which contains information about the direction of spin of the top quark and one which contains information about the spin of the top antiquark. The angular distribution of the decay products of a polarised top quark or antiquark is given by the following distribution:

$$
\frac{1}{\sigma} \frac{\mathrm{d} \sigma}{\mathrm{d} \cos \theta_{i}}=\frac{1}{2}\left(1+\alpha_{i} \cos \theta_{i}\right)
$$


Table 7.1: Spin analysing power of the different daughter particles of a spin up top quark. For a spin down or top antiquark the signs are reversed. Values taken from Reference [33].

\begin{tabular}{cccccc}
\hline & b-quark & $W^{+}$ & $\ell^{+}$ & $\overline{\mathrm{d}}$ - or $\overline{\mathrm{s}}$-quark & u- or c-quark \\
\hline$\alpha(\mathrm{LO})$ & -0.41 & 0.41 & 1. & 1. & -0.31 \\
$\alpha(\mathrm{NLO})$ & -0.39 & 0.39 & 0.998 & 0.93 & -0.31 \\
\hline
\end{tabular}

where $\theta_{i}$ is the angle between the direction of flight of the $i$-th decay product in the top quark rest frame and a reference direction $\hat{\mathbf{a}}$, often referred to as spin basis. The amount of information that a daughter particle carries is parameterised using the coefficient $\alpha_{i}$. This parameter is referred to as the spin analysing power of a daughter particle. While the angular distributions of all decay products of the top quark carry information about the direction of the spin, the sensitivity to the direction varies [33, 39]. The spin analysing power of the different daughter particles is listed in Table 7.1. The high analysing power of the charged lepton and the $\overline{\mathrm{d}}$ - or $\overline{\mathrm{s}}$-quark is due to the axial-vector nature of the $\mathrm{W}$ boson coupling.

The charged leptons carry the largest amount of information possible in their angular distribution. Furthermore they can easily be associated to the top quark or anti-quark depending on their charge. The down type quark carries as much information as the charged lepton, however, experimentally it is difficult to distinguish between down and up type quarks. Selecting, at random, one of the quarks from a hadronically decaying $\mathrm{W}$ boson the effective spin analysing power is $\alpha=(1-0.31) / 2=0.35$. Therefore events with a hadronically decaying $\mathrm{W}$ boson do not provide the same sensitivity as events where both $\mathrm{W}$ bosons decay to leptons. A further motivation for choosing the dilepton final state for this analysis.

Hadronic top quark pair production and decay parameters have been calculated to NLO QCD, in particular differential cross sections describing $\mathrm{t} \overline{\mathrm{t}}+X$ production in a general spin configuration [27]. The differential cross section with respect to $\cos \theta_{1}$ and $\cos \theta_{2}$ is an observable sensitive to spin 
correlation and is given by:

$$
\frac{1}{\sigma} \frac{d \sigma}{d \cos \theta_{1} d \cos \theta_{2}}=\frac{1}{4}\left(1-C \cos \theta_{1} \cos \theta_{2}+B_{1} \cos \theta_{1}+B_{2} \cos \theta_{2}\right),
$$

where $\sigma$ is the cross section for the channel under consideration and $C$ is a parameter between -1 and 1 . The parameters $B_{1}$ and $B_{2}$ will be discussed shortly. The value of $C$ depends on the strength of the spin correlation, and the choice of reference direction $\hat{\mathbf{a}}$. At the Tevatron the spin basis which corresponds to the largest value of $C$ is simultaneously the one which is simplest to construct:

$$
\hat{\mathbf{a}}=\hat{\mathbf{b}}=\hat{\mathbf{p}}
$$

where $\hat{\mathbf{p}}$ is the direction of flight of one of the colliding hadrons in the t $\bar{t}$ rest frame. This choice is referred to as the beam basis.

The two terms including the factors $B_{1}$ and $B_{2}$ in Equation 7.1 are zero in the SM as both the top quark and antiquark have no net polarisation [27]. In this analysis we will not consider them further, however, we will meet them again in Chapter 8, where we can use the top quark polarisation to distinguish new physics models which predict forward-backward asymmetries of equal value.

The degree of the correlation between the spins of top quark and antiquark $A$ is determined by the production mechanism and is defined by the fractional difference between the number of events where the spins of the top quarks are aligned and those where the top quarks spin have opposite alignment with respect to the spin basis:

$$
A=\frac{N_{\uparrow \uparrow}+N_{\downarrow \downarrow}-N_{\downarrow \uparrow}-N_{\uparrow \downarrow}}{N_{\uparrow \uparrow}+N_{\downarrow \downarrow}+N_{\downarrow \uparrow}+N_{\uparrow \downarrow}} .
$$

The coefficient $A$, which gives the strength of the correlation in production, is not measured directly in the distribution 7.1. Instead the observed correlation strength is given by the parameter $C=\left|\alpha_{i} \alpha_{j} A\right|$. The coefficients $\alpha_{i, j}$ are the spin analysing power of the top quark and antiquark decay product which is used to analyse its spin direction. To maximise the observable spin correlation the charged leptons are the optimal choice of decay 
product as they have the largest possible spin analysing power. At NLO in QCD $C=0.777$.

The angles $\theta_{1}$ and $\theta_{2}$ used in Equation 7.1 are calculated according to the following steps [27]:

1. Reconstruct the top quark and antiquark four momenta in the laboratory frame.

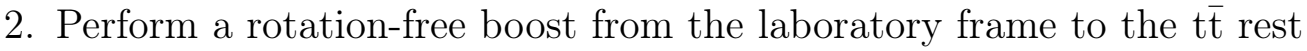
frame. Define the reference directions $\hat{\mathbf{a}}$ and $\hat{\mathbf{b}}$ in this frame.

3. Perform a rotation-free boost to the top quark and antiquark rest frame. Compute the direction $\hat{\mathbf{q}}_{1}$ of the positively charged lepton in the $t$ rest frame and the direction $\hat{\mathbf{q}}_{2}$ of the negatively charged lepton in the $\overline{\mathrm{t}}$ rest frame. Finally, compute $\cos \theta_{1}=\hat{\mathbf{a}} \cdot \hat{\mathbf{q}}_{1}$ and $\cos \theta_{2}=\hat{\mathbf{b}} \cdot \hat{\mathbf{q}}_{2}$.

The distribution $\cos \theta_{1} \cos \theta_{2}$ calculated according to this prescription is shown in Figure 7.1, here parton level information was used. Due to the two neutrinos in the dilepton final state the four vectors of the top quark and antiquark are not easily available. In Section 7.2 the method used to reconstruct them is given.

\subsection{Reconstruction}

The calculation of the angular correlations described in Section 7.1 requires the reconstruction of the four vectors of the top quark and anti-quark. This is complicated by the fact that each event contains two neutrinos which escape detection. The Neutrino Weighting Technique ( $v W T)$ was first developed to perform measurements of the mass of the top quark which requires a full reconstruction of the four momentum [8]. The difference here is that the knowledge of the top quark mass can be used to provide an additional constraint.

In the dilepton channel the final state consists of two charged leptons, two jets originating from $\mathrm{b}$ quarks and two neutrinos. The kinematics of the event are fully determined by the four vectors of these objects. As the masses of 


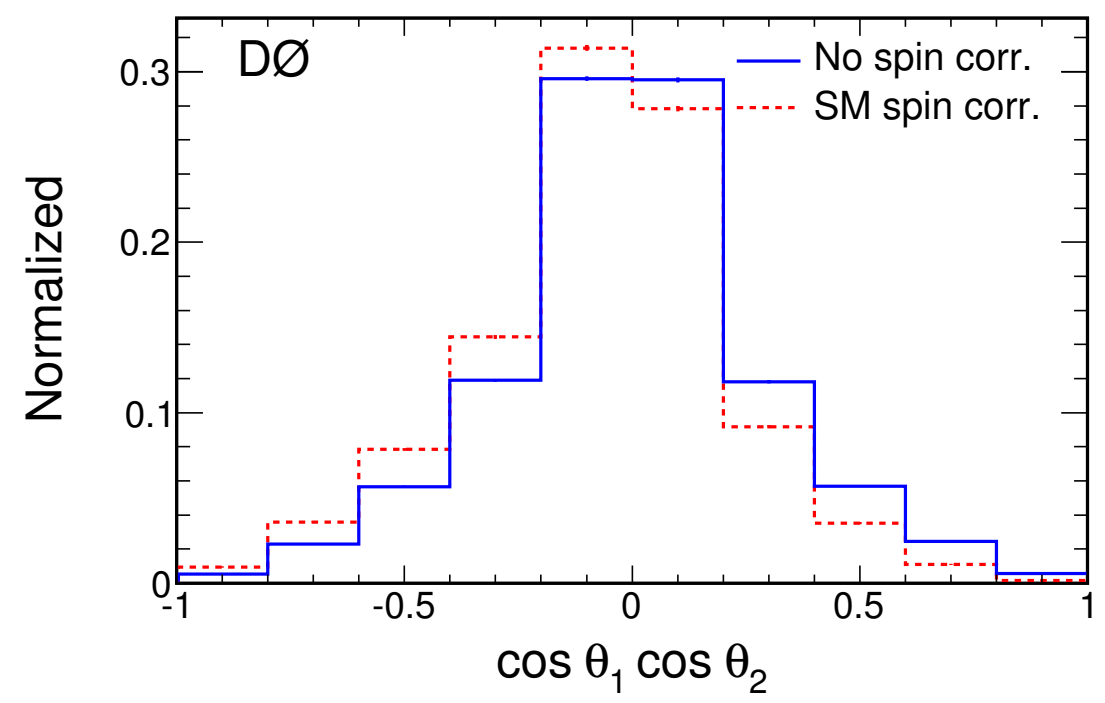

Figure 7.1: This plot shows distribution 7.1 calculated using parton level objects, where the four vectors of the top quarks are known exactly. Both MC@NLO with and without standard model spin correlations is shown.

these particles are known this reduces to 18 independent quantities. However in the detector only the momenta and energies of the charged leptons and jets are measured. The neutrinos escape detection. Four further constraints are provided by assuming that the events originate from top quark pair decays:

- The invariant mass of each lepton and neutrino pair add to the mass of the $\mathrm{W}$ boson of $80.4 \mathrm{GeV}$,

- the invariant mass of the lepton, neutrino and jet equal the mass of the top quark assumed to be $172.5 \mathrm{GeV}$.

The measured momenta, energies and four constraints provide 16 quantities whilst 18 are needed. The missing two quantities are provided by integrating over the pseudo-rapidities, $\eta$, of the neutrinos. The neutrino $\eta$ distributions are taken from Monte Carlo. From these distributions ten $\eta$ values are chosen in steps of equal probability for both neutrinos and anti-neutrinos. As a result the step size in $\eta$ reduces in the central region which is more likely. This yields a total of 100 pairs of assumed $\eta$ values. For each pair of assumed 


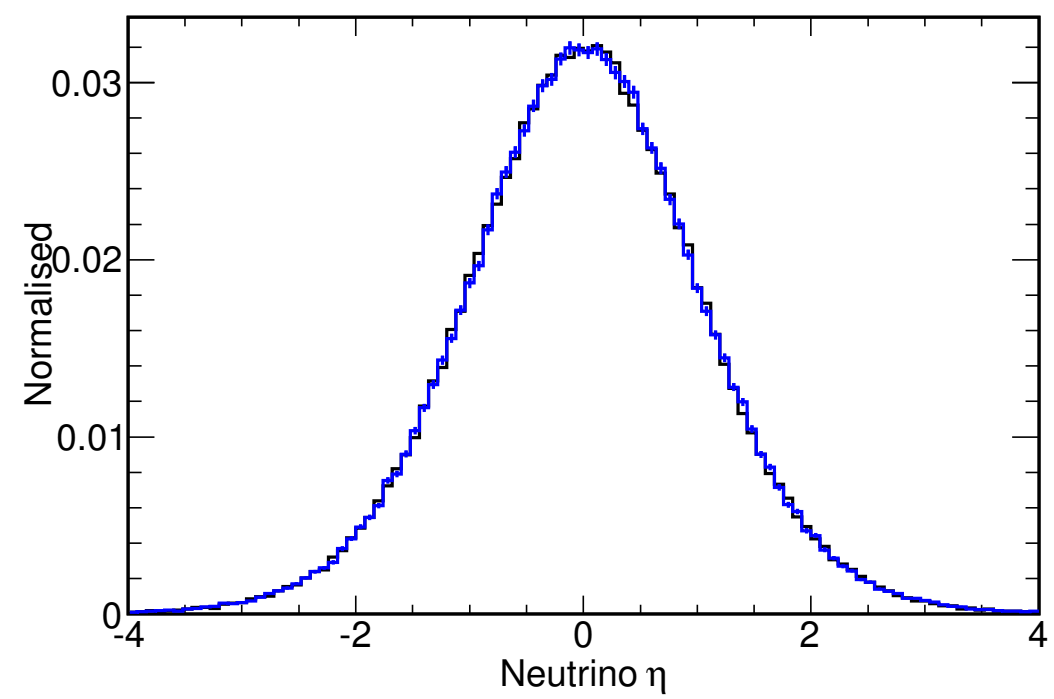

Figure 7.2: Comparing the neutrino $\eta$ distributions for MC@NLO simulated events with and without spin correlations.

$\eta$ values the equations describing the kinematics of the event can be solved. As these equations are quadratic for the top quark and top antiquark there are up to four solutions per set of assumed $\eta$ pairs.

As the neutrino $\eta$ distributions are taken from $\mathrm{MC}$ it is important to check that they do not depend on the presence of t $\bar{t}$ spin correlations as this could bias the reconstruction. In Figure 7.2 the rapidity distribution is shown for both cases, with and without $t \bar{t}$ spin correlations, with no difference visible between the two.

An additional complication in the reconstruction arises from the unknown lepton-jet pairing. The charge of the lepton clearly identifies whether it originated from a top quark or anti-quark. For the jets it is very difficult to distinguish between one originating from a quark and an antiquark. As a result both possible pairings of lepton and jet are tried and the possible number of solutions for each neutrino $\eta$ pair increases to eight.

If a solution exists for a given neutrino $\eta$ assumption any observable can be calculated for the event as all quantities needed to specify the final state 
are known. Once the neutrino four vector has been calculated the parent top quark is reconstructed assuming a $\mathrm{t} \rightarrow \mathrm{b} \ell^{+} \nu$ decay. It is then straight forward to calculate the angles $\theta_{1}$ and $\theta_{2}$.

Each solution is assigned a weight to characterise how well it agrees with the $t \bar{t}$ hypothesis, by comparing the $x$ and $y$ components of the measured missing transverse energy with the sum of the $p_{x}$ and $p_{y}$ components of the neutrinos reconstructed using each neutrino $\eta$ assumption. The weight is defined as:

$$
w=\exp \left(-\frac{\left(E_{x}^{\text {miss }}-p_{x}^{\nu}-p_{x}^{\bar{\nu}}\right)}{2 \sigma^{2}}\right) \cdot \exp \left(-\frac{\left(E_{y}^{\text {miss }}-p_{y}^{\nu}-p_{y}^{\bar{\nu}}\right)}{2 \sigma^{2}}\right)
$$

where $p_{x, y}^{\nu}$ are the $x$ and $y$ components of the neutrino momentum, $p_{x, y}^{\bar{\nu}}$ are the $x$ and $y$ components of the anti-neutrino momentum, $E_{x, y}^{\text {miss }}$ are the $x$ and $y$ components of the measured missing transverse energy and $\sigma$ is the $\mathbb{E}_{T}$ resolution which is taken as $10 \mathrm{GeV}$.

The procedure so far does not account for possible mismeasurement of objects in the detector. Slight variations in the measured quantities can make the set of equations describing the final state kinematics unsolvable. To increase the efficiency of solving the equations and to account for the finite resolution the kinematics of the final state objects (jets and leptons) are varied within their respective resolutions and a solution is attempted for each variation. The resolution of jets, electrons and muons is parameterised as a function of the objects $p_{T}$ and $\eta$.

The energy resolution of jets is parameterised by a double gaussian, whose parameters depend on the $p_{T}, \eta$ of a jet and whether or not a muon associated with the jet. The muon indicates that the jet contained a $b$ quark. The parameterisation is described in full detail in Reference [45].

The electron energy resolution is studied in $\mathrm{Z} \rightarrow$ ee events and taken from Reference [44]. The energy is determined from the measurement in the calorimeter, and therefore the resolution improves as the energy increases. For muons the momentum is determined from the curvature of the track reconstructed in the tracking detector. As the momentum increases, so does the 
Table 7.2: Efficiency for finding at least one solution per event for different MC samples. The $Z \rightarrow$ ee, $Z \rightarrow \mu \mu$ and $Z \rightarrow \tau \tau$ backgrounds are shown combined, as are the diboson and instrumental backgrounds. In the last column we give the value observed in data. The statistical uncertainties are $\approx 1 \%$.

\begin{tabular}{cccccc}
\hline $\mathrm{t} \overline{\mathrm{t}}$ & $Z$ & Diboson & Instrumental & Total & Observed \\
\hline 0.96 & 0.82 & 0.90 & 0.82 & 0.92 & 0.91 \\
\hline
\end{tabular}

curvature, leading to a straighter track. As a result the momentum resolution increases as the muon momentum increases. More details of the determination of the resolution and parameterisation are given in Reference [47].

Table 7.2 shows the efficiency for finding at least one solution in both signal and background MC events.

In Figure 7.3 the weight distribution after iterating over all assumptions is shown for one example event. If there is at least one solution for an event, the weighted average of all solutions is used as estimate of the true value of the observable.

\subsection{Template fitting}

The spin correlation strength $C$ is extracted from the data by first reconstructing the product $\cos \theta_{1} \cos \theta_{2}$ for each event in data and simulation. By using t $\overline{\mathrm{t}}$ simulation with and without spin correlation $(C=0)$ we can construct a template for the expected distribution of events in data for each of the two cases as well as for mixtures of the two. A template is constructed for each background process as well. These templates are then used in a binned maximum likelihood fit to extract the fraction $f_{\mathrm{SM}}$ of standard model like $t \overline{\mathrm{t}}$ events.

Using a template with vanishing spin correlations, with $N_{0}^{(i)}$ entries in bin $i$, a template with SM spin correlations and $N_{\mathrm{SM}}^{(i)}$ entries and $N_{\mathrm{bg}}^{(i)}$ backgrounds events, the fraction of the SM contribution $f_{\mathrm{SM}}$ is extracted by fitting

$$
N_{i}=f_{\mathrm{SM}} N_{\mathrm{SM}}^{(i)}+\left(1-f_{\mathrm{SM}}\right) N_{0}^{(i)}+N_{\mathrm{bg}}^{(i)}
$$




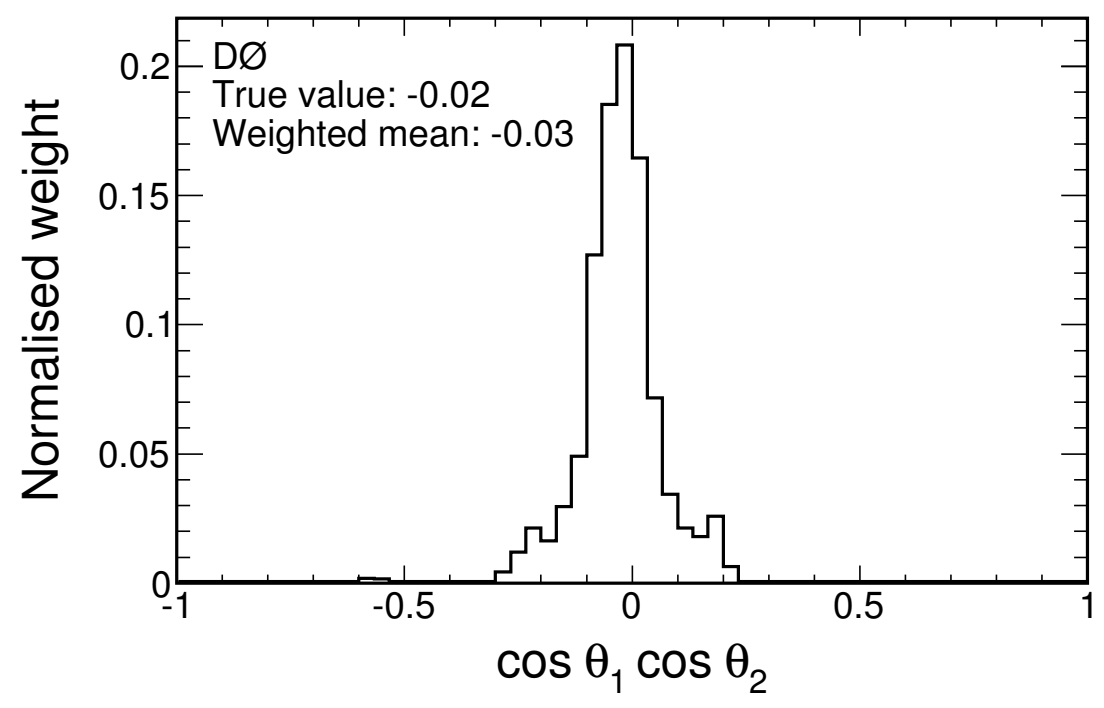

Figure 7.3: Weight distribution as function of $\cos \theta_{1} \cos \theta_{2}$ for an example t $\bar{t}$ MC event. The weighted mean of all solutions is close to the value calculated using the generator level information of the event.

to the data.

To create the templates, histograms of the $\cos \theta_{1} \cos \theta_{2}$ distribution are created. During the fit the parameter $f_{\mathrm{SM}}$ is not constrained to the physically allowed region. For values far outside it one starts subtracting one of the signal templates from the other. This can lead to a negative predicted bin content. The binning of the templates is chosen to avoid this, except for values of $f_{\mathrm{SM}}$ extremely far from the physically allowed region. This leads to wider bins at the edges of the distribution where the difference between the templates with and without SM spin correlations is large compared to the background contribution. The same binning is used in all channels, namely one bin from -1.0 to -0.4 , then eight bins of width 0.1 , followed by a final bin from +0.4 to +1.0 . The templates used during the fit are shown in Figures 7.4-7.6.

Agreement with the SM is found if $f_{\mathrm{SM}}$ is measured to be 1 . Because the distribution (7.1) is linear in the coefficient $C, f_{\mathrm{SM}}$ can be translated into $C$ as follows: $C=0.777 \cdot f_{\mathrm{SM}}$. 


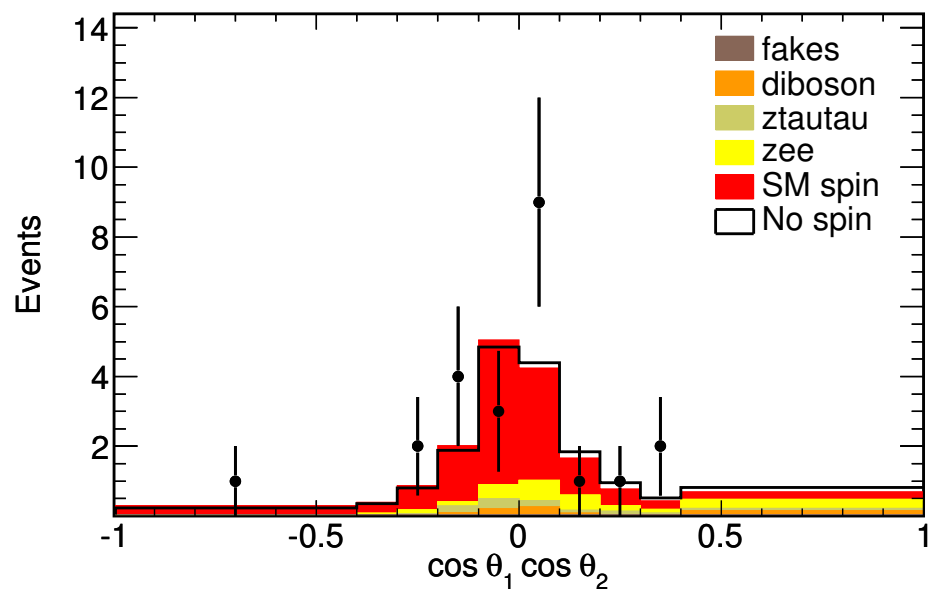

(a)

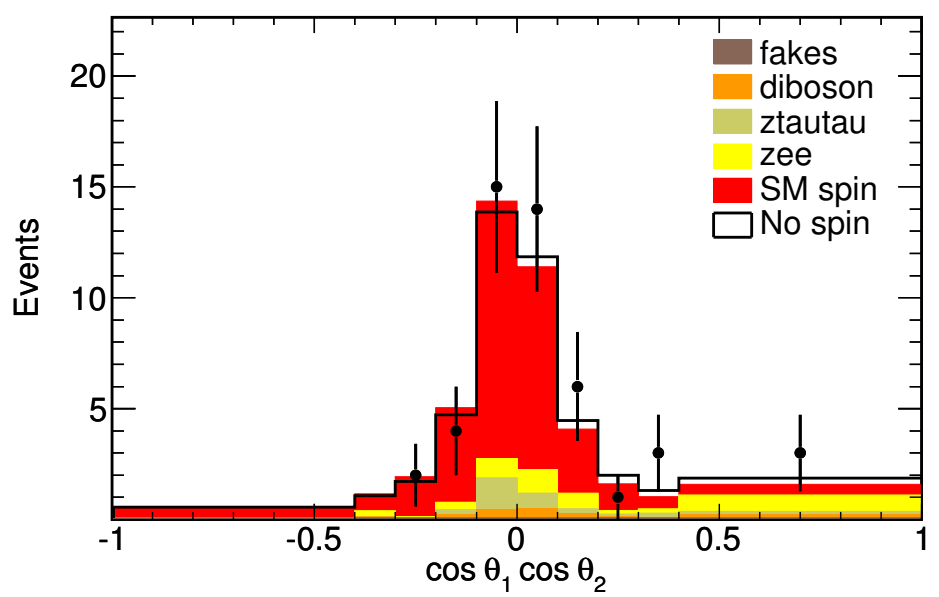

(b)

Figure 7.4: The $\cos \theta_{1} \cos \theta_{2}$ distributions used as templates to extract the spin correlation strength $C$. Data and simulation for the dielectron channel for (a) Run IIa and (b) Run IIb are shown. 


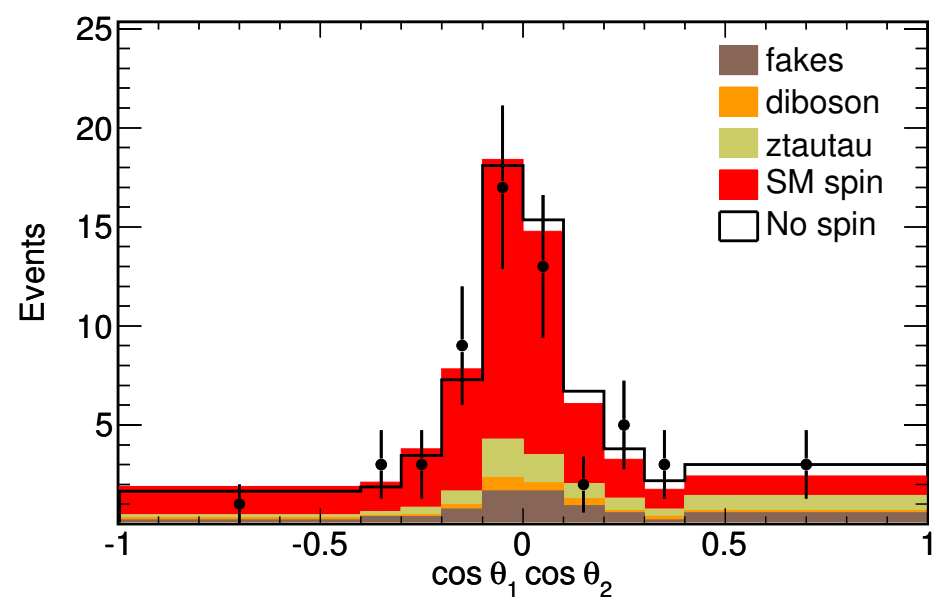

(a)

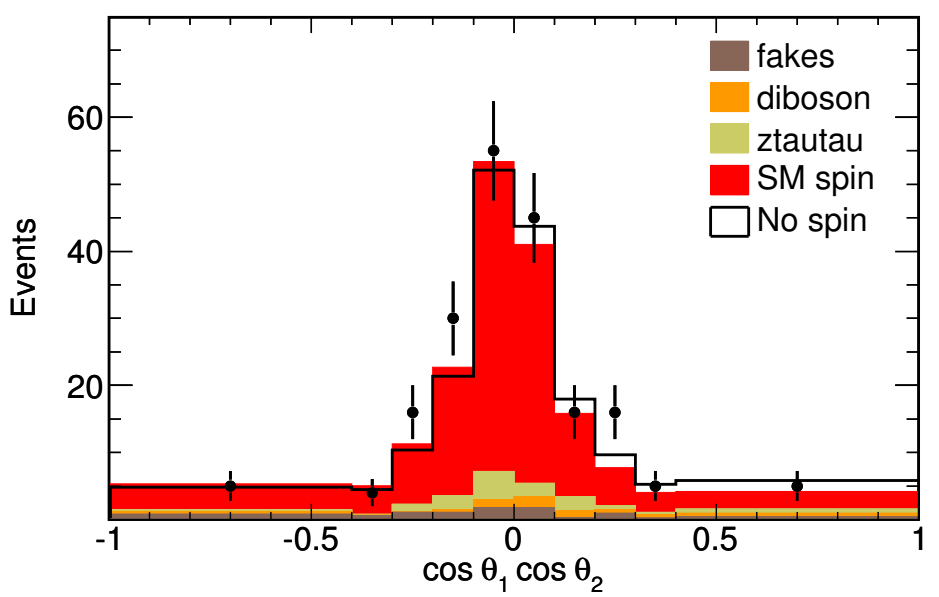

(b)

Figure 7.5: The $\cos \theta_{1} \cos \theta_{2}$ distributions used as templates to extract the spin correlation strength $C$. Data and simulation for the e $\mu$ channel for (a) Run IIa and (b) Run IIb are shown. 


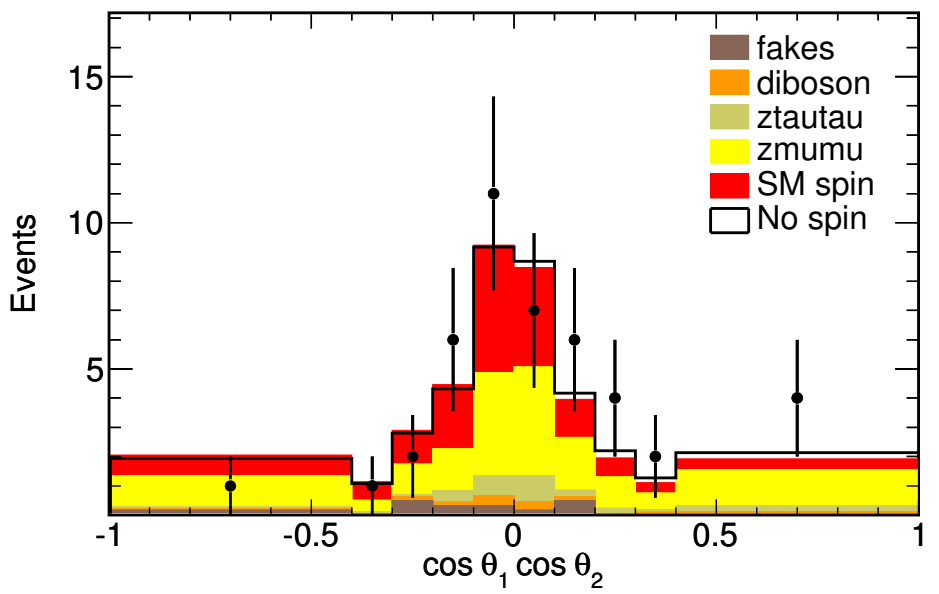

(a)

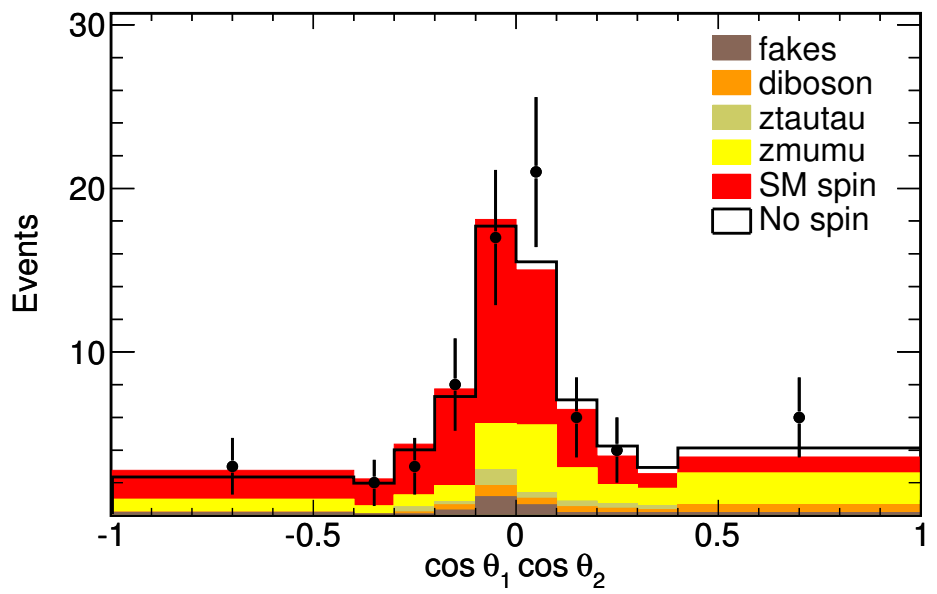

(b)

Figure 7.6: The $\cos \theta_{1} \cos \theta_{2}$ distributions used as templates to extract the spin correlation strength $C$. Data and simulation for the dimuon channel for (a) Run IIa and (b) Run IIb are shown. 
Systematic uncertainties are accounted for in the fit by a nuisance parameter for each independent source of systematic uncertainty. Each nuisance parameter is constrained by a Gaussian probability density function centred on zero and width one. Correlations between channels are taken into account by using the same nuisance parameter for a source of systematic uncertainty across channels.

The negative logarithm of the likelihood function

$$
\mathcal{L}=\prod_{i=1}^{N} \mathcal{P}\left(n_{i}, N_{i}\right) \times \prod_{k=1}^{K} \mathcal{G}\left(\nu_{k} ; 0,1\right)
$$

is minimised with $\mathcal{P}\left(n_{i}, N_{i}\right)$ representing the Poisson probability to observe $n$ events when $N_{i}$ events are expected and $\mathcal{G}\left(\nu_{k} ; 0,1\right)$ is the normal probability of the nuisance parameter $k$ to take the value $\nu_{k}$. The expected number of events is given by Equation 7.2 and is a function of $f_{\mathrm{SM}}$ the parameter of interest and the $t \bar{t}$ cross section. The product in Equation 7.3 runs over the $N$ bins of the template and the $K$ independent systematic uncertainties. In order to reduce the dependency of $f_{\mathrm{SM}}$ on the normalisation of the signal the $t \bar{t}$ cross section is extracted simultaneously. As we will see later this measurement of the cross section is of comparable sensitivity to a dedicated cross section measurement.

In order to include systematic uncertainties in the predicted number of events in Equation $7.2 N_{\mathrm{SM}}^{(i)}, N_{0}^{(i)}$ and $N_{\mathrm{bg}}^{(i)}$ are modified to include a term for each nuisance parameter $\nu_{k}$. The procedure is the same for each of the three numbers, so the explanation will be given in general terms. For each bin the yield $N_{i}^{\prime}$ is calculated by varying a source of systematic uncertainty by one standard deviation, in addition to calculating the nominal yield $N_{i}$. The expression for the number of predicted events $n_{i}$ for a bin including the nuisance parameter treatment for systematic effects is then:

$$
n_{i}=N_{i}+\nu\left(N_{i}-N_{i}^{\prime}\right)
$$

To allow for a difference between the plus one and minus one standard 
deviation variation, the expression for $n$ is extended to:

$$
n_{i}=N_{i}+\nu \begin{cases}\left(N_{i}-N_{i}^{\text {up }}\right), & \nu>0 \\ \left(N_{i}-N_{i}^{\text {down }}\right), & \nu<0\end{cases}
$$

where $N_{i}^{\text {up }}\left(N_{i}^{\text {down }}\right)$ is the yield calculated for the plus (minus) one standard deviation variation. When considering all $K$ sources of systematic uncertainty the expression for $n$ extends to:

$$
n_{i}=N_{i}+\sum_{k=1}^{K} \nu_{k} \begin{cases}\left(N_{i}-N_{i}^{k, \text { up }}\right), & \nu_{k}>0 \\ \left(N-N_{i}^{k, \text { down }}\right), & \nu_{k}<0 .\end{cases}
$$

The physically allowed range of the parameter $f_{\mathrm{SM}}$ is limited to the range $[-1,1]$. The expected statistical uncertainty of the measurement is large compared to this range. As a result best fit values of $f_{\mathrm{SM}}$ which lie outside the physically allowed region are likely. Instead of restricting the parameters of interest to this physically allowed region we use the prescription of Feldman and Cousins [42] to extract the final confidence band.

\subsection{Feldman-Cousins method}

A frequentist confidence interval is constructed so that it contains the unknown, true value of the parameter of interest, $\mu_{t}$, in a fraction $\alpha$ of experiments. The size of the confidence band depends on the chosen confidence level (C.L.), referred to as $\alpha$. A confidence interval $\left[\mu_{1}, \mu_{2}\right]$ is chosen so that $P\left(\mu \in\left[\mu_{1}, \mu_{2}\right]\right)=\alpha$, the ends of the interval $\mu_{1}$ and $\mu_{2}$ being functions of the parameter, $x$, measured in the experiment. Generally the method of Neyman [66] is used to construct confidence belts for one unknown parameter $\mu$ and one measured quantity $x$. For each value of $\mu$ one choses an interval $\left[x_{1}, x_{2}\right]$ such that $P\left(x \in\left[x_{1}, x_{2}\right] \mid \mu\right)=\alpha$. The interval $\left[x_{1}, x_{2}\right]$ is referred to as the acceptance region. To fully define the interval two parameters are required. The most common choices for this second parameter are to choose 
to construct an upper confidence limit such that

$$
P\left(x<x_{1} \mid \mu\right)=1-\alpha
$$

or to construct a central confidence interval

$$
P\left(x<x_{1} \mid \mu\right)=P\left(x>x_{2} \mid \mu\right)=\frac{1-\alpha}{2} .
$$

Both choices provide confidence bands with the correct coverage only if the choice of which type of interval to construct is made before looking at the data. For a parameter close to a physical boundary it is tempting to choose to report a central confidence interval if both ends for this particular measured value of $x$ are within the physically allowed region and otherwise report an upper limit. Constructing confidence bands in this way leads to incorrect coverage.

An alternative way of providing the second parameter required to construct the acceptance region is to define a function

$$
R(x)=\frac{P(x \mid \mu)}{P\left(x \mid \mu_{\text {best }}\right)}
$$

where $\mu_{\text {best }}$ is the best estimate of $\mu_{t}$ for a given value of $x$. If $x$ is in the physically allowed region then $\mu_{\text {best }}=x$. However, if $x$ is in the unphysical region then $\mu_{\text {best }}$ is equal to the value of $x$ at the boundary. For the measurement of $f_{\mathrm{SM}}$ the choices are:

$$
\mu_{\text {best }}= \begin{cases}-1, & f_{\mathrm{SM}}<-1 \\ f_{\mathrm{SM}}, & -1 \leq f_{\mathrm{SM}} \leq+1 \\ +1, & +1<f_{\mathrm{SM}} .\end{cases}
$$

The two parameters defining the interval $\left[x_{1}, x_{2}\right]$ for a given value of $\mu$ are:

$$
\begin{gathered}
R\left(x_{1}\right)=R\left(x_{2}\right) \\
\int_{x_{1}}^{x_{2}} P(x \mid \mu) \mathrm{d} x=\alpha .
\end{gathered}
$$


The probability density function $P(x \mid \mu)$ is determined by generating many pseudo experiments for each value of $\mu$. For a given value of $\mu$ the values of $x$ with the largest $R(x)$ are added to the acceptance region until the sum $P(x \mid \mu)$ is equal to or exceeds $\alpha$.

Confidence intervals constructed following this prescription are equal to those obtained from constructing a central confidence band far away from the physical boundaries. As the measured value of the parameter approaches a boundary a smooth transition from two sided confidence intervals to a one sided limit occurs. As a result they provide the correct coverage, compared to the undercoverage caused by choosing to report a one or two sided confidence belt based on the data.

\subsection{Matrix element based method}

The spin correlations measurement in the dilepton final state is limited by the statistical uncertainty due to the size of the sample. Comparing the sensitivity of the $v \mathrm{WT}$ method to the matrix element method, both of which have been used to measure the top quark mass [13, 8], the application of this method to the measurement of spin correlations promises significant improvements to the statistical uncertainty.

The matrix element method has previously been applied to measurements of the top quark mass and the helicity fraction of the $W$ boson in $t \bar{t}$ decays. It relies on a free parameter in the matrix element which controls the effect under study. For example the top quark mass is a parameter in the matrix element describing top quark pair production. No such parameter exists for tẼ spin correlations.

Therefore the matrix element method is modified slightly. Instead of relying on a parameter in the matrix element it is used to distinguish two hypotheses: SM like spin correlation and no correlation. For each of these hypotheses a matrix element can be formulated. The signal probability $P_{\text {sgn }}$ is calculated separately for both hypotheses: SM like spin correlation $(H=1)$ and no correlation $(H=0)$. Templates are constructed for the ratio $R$ 
defined as:

$$
R=\frac{P_{\mathrm{sgn}}(H=1)}{P_{\mathrm{sgn}}(H=0)+P_{\mathrm{sgn}}(H=1)}
$$

which are used instead of the templates in $\cos \theta_{1} \cos \theta_{2}$ used in the $v \mathrm{WT}$ measurement.

Once templates using this improved observable have been constructed, the procedure for the template fit, interpretation of the best fit value within the physically allowed range, sources of systematic uncertainty considered and their incorporation in the fit are exactly as for the preceding analysis.

The signal probability $P_{\text {sgn }}$ used is given by

$$
P_{\text {sgn }}(x ; H)=\frac{1}{\sigma} \int \mathrm{d} \epsilon_{1} \mathrm{~d} \epsilon_{2} f_{\mathrm{PDF}}\left(\epsilon_{1}\right) f_{\mathrm{PDF}}\left(\epsilon_{2}\right) \frac{(2 \pi)^{4}|M(y, H)|^{2}}{\epsilon_{1} \epsilon_{2} s} \mathrm{~d} \Phi_{6} W(x, y)
$$

where $\sigma$ denotes the cross section, $\epsilon_{1}\left(\epsilon_{2}\right)$ is the energy fraction of the (anti)proton carried by the incoming (anti)quark, $f_{\mathrm{PDF}}$ are the parton distribution function, the centre-of-mass energy squared $s, \mathrm{~d} \Phi_{6}$ is an element of the 6-body phase space and $M(y, H)$ is the leading order matrix element. The detector resolution is taken into account by the transfer function $W(x, y)$ which gives the probability of a partonic state $y$ giving rise to a measured state $x$ in the detector.

While the spin correlation strength $C$ is not a parameter present in the matrix element, the matrix element neglecting spin correlations and the matrix element including SM spin correlations can be written as [61]:

$$
\sum|M|^{2}=\frac{(1+H)}{2} \frac{g_{s}^{4}}{9} F \bar{F}\left(2-\beta^{2} s_{q t}^{2}\right)-H \frac{g_{s}^{4}}{9} F \bar{F} \Delta
$$

where $\beta$ is the velocity of the top quarks in the $t \bar{t}$ rest frame, $g_{s}$ is the strong coupling constant and $s_{q t}$ denotes the sine of the scattering angle of

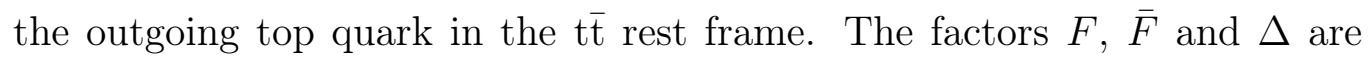
related to the kinematics of the top quark and antiquark. The sine (cosine) of the angle between particles $x$ and $y$ in the rest frame of the $W$ boson is 
abbreviated as $s_{x y}\left(c_{x y}\right)$. For leptonic $W$ boson decays:

$$
\begin{aligned}
& F=\frac{g_{w}^{4}}{4}\left[\frac{m_{b \bar{\ell} \nu}^{2}-m_{\bar{\ell} \nu}^{2}}{\left(m_{b \bar{\ell} \bar{\nu}}^{2}-m_{t}^{2}\right)^{2}+\left(m_{t} \Gamma_{t}\right)^{2}}\right]\left[\frac{m_{b \bar{\ell} \nu}^{2}\left(1-c_{b \bar{\ell}}^{2}\right)+m_{\bar{\ell} \nu}^{2}\left(1+c_{b \bar{\ell}}\right)^{2}}{\left(m_{\bar{\ell} \nu}^{2}-m_{W}^{2}\right)^{2}+\left(m_{W} \Gamma_{W}\right)^{2}}\right] \\
& \bar{F}=\frac{g_{w}^{4}}{4}\left[\frac{m_{\bar{b} \ell \bar{\nu}}^{2}-m_{\ell \bar{\nu}}^{2}}{\left(m_{\bar{b} \ell \bar{\nu}}^{2}-m_{t}^{2}\right)^{2}+\left(m_{t} \Gamma_{t}\right)^{2}}\right]\left[\frac{m_{\bar{b} \ell \bar{\nu}}^{2}\left(1-c_{b \bar{\ell} \bar{l}}^{2}+m_{\ell \bar{\nu}}^{2}\left(1+c_{\bar{b} \ell}\right)^{2}\right.}{\left(m_{\ell \bar{\nu}}^{2}-m_{W}^{2}\right)^{2}+\left(m_{W} \Gamma_{W}\right)^{2}}\right]
\end{aligned}
$$

where the invariant mass of two (three) particles is denoted as $m_{x y}\left(m_{x y z}\right)$, the mass and width of the $W$ boson are $m_{W}$ and $\Gamma_{W}$, the mass and width of the $t$ quark are $m_{t}$ and $\Gamma_{t}$, and the weak coupling constants is $g_{w}$. The factor $\Delta$ is given by

$$
\Delta=\frac{\left(1-c_{\bar{\ell} q} c_{\ell \bar{q}}\right)-\beta\left(c_{\ell \bar{t}}+c_{\bar{\ell} t}\right)+\beta c_{q t}\left(c_{\bar{\ell} q}+c_{\ell \bar{q}}\right)+\frac{1}{2} \beta^{2} s_{q t}^{2}\left(1-c_{\bar{\ell} \ell}\right)}{\gamma^{2}\left(1-\beta c_{\bar{\ell} \ell}\right)\left(1-\beta c_{\ell \bar{t}}\right)} .
$$

The signal probability $P_{\text {sgn }}$ depends on the four momenta of all final state particles, not all of which are measured by the detector. To reduce the dimensionality of the integration quantities which are assumed to be well measured are not integrated over: electron energy, the jet and charged lepton directions. The number of variables is further reduced by assuming that the $\mathrm{t} \overline{\mathrm{t}}$ system has no net transverse momentum. This leaves an integral over six variables.

The following trade offs need to be considered when choosing which six variables to integrate over. An integrand which is strongly peaked as a function of the integration variables will reduce the time required to compute the integral. However, the variable transformation required to compute the four momenta of the partons in the event should not be too complicated to calculate as otherwise the time gained from transforming the variables will be lost. Finally, the integration variables should be uncorrelated. The following six integration variables were chosen:

$$
\left|\vec{p}_{b 1}\right|,\left|\vec{p}_{b 2}\right|, p_{\nu_{1}}^{x}-p_{\nu_{2}}^{x},, p_{\nu_{1}}^{y}-p_{\nu_{2}}^{y}, m_{W_{1}}^{2}, m_{W_{2}}^{2}\left[, q_{\mu} / p_{\mu}^{T}\right]
$$

After performing the integration for each event under both hypotheses, 


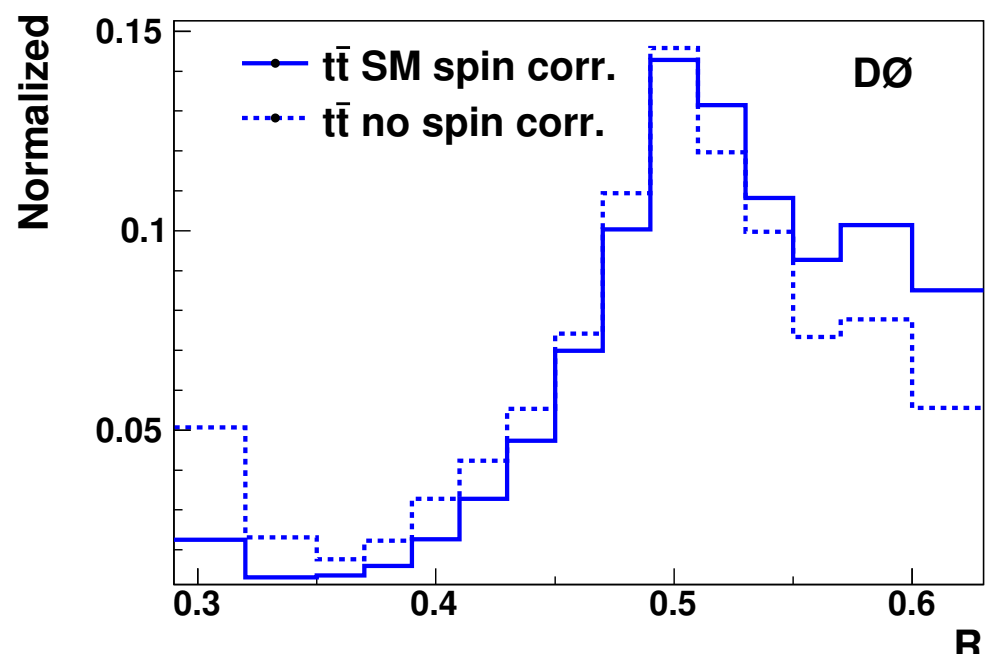

Figure 7.7: Comparison of the SM spin correlation and no spin correlation hypotheses using $R$ as discrimminating variable. Parton level objects are used to calculate $P_{\text {sgn }}$. The first and last bin contain the under- and overflow.

the ratio $R$ is calculated and templates built. The next steps of this analysis are to fit the templates to the data and extract $f_{\mathrm{SM}}$. Followed by estimating the size of the systematic uncertainties. The same methods as for the $v \mathrm{WT}$ based analysis are used. However, the template fit uses $R$ as discriminating variable instead of $\cos \theta_{1} \cos \theta_{2}$. The distribution of $R$ for the case of SM spin correlation and no correlation are shown in Figure 7.7.

\subsection{Systematic uncertainties}

No scientific measurement is complete without considering possible sources of systematic uncertainty. They can be broadly split into two separate categories. Flat systematics are those effecting the absolute normalisation of a sample only. Those effecting the shape of the distribution used in the template fit are referred to as shape changing systematics.

All systematic uncertainties are considered to be correlated between the different final states in the same run period. The following systematic uncertainties are considered to be correlated between the two run periods: jet 
energy scale, jet energy resolution, jet identification, electron and muon identification, and the uncertainty on the trigger efficiency.

Each independent source of systematic uncertainty is modelled by a nuisance parameter in the template fit procedure of Section 7.3. Correlated systematic uncertainties are modelled using the same nuisance parameter in the samples in which they are correlated. The following uncertainties are considered as flat systematics.

\section{$Z+$ jets normalisation}

An additional uncertainty of $10 \%$ is assigned to the yield of the $Z+$ jets MC samples as a result of the mismodelling found at the stage of the basic selection.

\section{Trigger}

The simulation of the D0 detector does not include an implementation of the triggers. Instead the efficiencies of the triggers are derived in data, and then applied to the simulation. The efficiencies are varied by one standard deviation in either direction.

\section{Electron and muon identification}

The efficiency for identifying electrons differs in data and simulation. To improve the modelling a scale factor is derived from the data which is parameterised as a function of $(\eta, \phi)$ of the electron. An alternative parameterisation as a function of $\left(\eta, p_{T}\right)$ is also derived. The sum of the difference between the two parameterisations and the statistical uncertainty on the scale factors is used as systematic variation.

To evaluate the effect shape changing systematic uncertainties have on the measurement 1000 pseudo experiments are constructed. In the pseudo experiments the expected number of events in a bin is first varied by multiplying 
the variation in expected number of events one obtains by varying the source of systematic uncertainty by one standard deviation by a gaussian random number centred on zero and width equal to one. This gaussian random number is shared for all bins in a pseudo experiments in order to take into account the bin to bin correlations.

\section{Jet energy scale}

Jet energies are measured in the calorimeter and are corrected for the response of the calorimeter, energy deposited outside the jet reconstruction cone, and for energy from underlying event activity within the jet cone. As a result the systematic uncertainty on the jet energy calibration has two parts. A statistical component arising from the limited number of MC and data events used to derive it and a systematic component arising from systematic uncertainties of the tools used in deriving it. The effect of the JES uncertainty on this analysis is determined by varying the JES calibration up and down by the quadrature sum of these two components.

\section{Heavy quark jet energy scale}

This uncertainty takes into account the difference between the nominal inclusive JES and the JES for b-hadrons. This later JES has been evaluated to be $1.8 \%$ smaller than the nominal JES. The systematic uncertainty is obtained by shifting the nominal JES down by this amount.

\section{Jet identification}

The uncertainty on the jet reconstruction and identification efficiency is determined by reducing the scale factor applied to MC by one standard deviation. The uncertainty is then symmetrised.

\section{Jet energy resolution}

The uncertainty on the jet energy resolution (JER) is determined by varying the JER correction up and down by one standard deviation in MC events. 


\section{Vertex confirmation}

In Run IIb jets are required to contain at least two tracks associated to the primary vertex. The scale factor applied to the MC efficiency is varied by its one standard deviation uncertainty.

\section{Parton density function}

This uncertainty is evaluated by using each one of the 20 errors sets provided by the CTEQ61M PDF. The total uncertainty due to PDFs is the quadrature sum of difference in the best fit value of each error set.

\section{Background reweighting}

After applying the basic selection criteria some mismodelling of the $\cos \theta_{1} \cos \theta_{2}$ distribution is observed in the dielectron and dimuon channels. To estimate the size of the effect of such a mismodelling the $Z+$ jets MC events are reweighted such that they match the data perfectly. The difference between applying this reweighting and not applying it is taken as size of the systematic uncertainty. This uncertainty is only applied to the $\cos \theta_{1} \cos \theta_{2}$ based analysis.

\section{MC template statistics}

The samples of simulated events used to derive the templates used in the fit are of finite size. The estimate of the predicted number of events in each bin is only known with finite precision. To take this effect into account the fit to data is repeated 1000 times each time varying the templates. Each time the data are fitted each bin is varied from its nominal value by adding a random number drawn from a gaussian distribution with mean equal to the expected number of events and width equal to the statistical uncertainty on this predicted number of events. The width of the distribution of best fit values obtained is used as estimate of this systematic uncertainty.

The measurement is dominated by the size of the data sample, the largest systematic uncertainty arises from the finite size of the MC samples. The size 
Table 7.3: Summary of uncertainties on $C_{\text {meas }}$ using the $v$ WT reconstruction technique.

\begin{tabular}{ccc}
\hline Source & $+\mathrm{SD}$ & $-\mathrm{SD}$ \\
\hline Muon identification & 0.01 & -0.01 \\
Electron identification and smearing & 0.01 & -0.01 \\
PDF & 0.02 & -0.01 \\
Top mass & 0.01 & -0.01 \\
Triggers & 0.02 & -0.02 \\
Opposite charge requirement & 0.00 & -0.00 \\
Jet energy scale & 0.01 & -0.01 \\
Jet reconstruction and identification & 0.06 & -0.06 \\
Normalisation & 0.02 & -0.02 \\
Monte Carlo statistics & 0.02 & -0.02 \\
Instrumental background & 0.00 & -0.00 \\
Spin background model & 0.03 & -0.04 \\
Luminosity & 0.03 & -0.03 \\
Other & 0.01 & -0.01 \\
Template statistics & 0.07 & -0.07 \\
\hline Total systematic uncertainty & 0.11 & -0.11 \\
Statistical uncertainty & 0.38 & -0.40 \\
\hline
\end{tabular}

of the systematic uncertainties for $C_{\text {meas }}$ summarised in Tables 7.3. For the matrix element based method the statistical uncertainty is reduced by $30 \%$. However, even with this large improvement the size of the statistical uncertainty still dominates the combined statistical and systematic uncertainties. An overview of the systematic uncertainties are listed in Table 7.4.

\subsection{Results}

The best fit value for $C_{\text {meas }}$ from the fit described in Section 7.3 can lie outside the physical region due to statistical fluctuations. Figure 7.8 shows the distribution for $\cos \theta_{1} \cos \theta_{2}$ for background, te signal with NLO QCD spin correlation, and the prediction for $t \bar{t}$ signal without spin correlation.

Using the Feldman-Cousins prescription described in Section 7.4 a physically meaningful value of $C$ can be extracted for any value of $C_{\text {meas }}$. For 
Table 7.4: Summary of uncertainties on $f_{\mathrm{SM}}$ extracted in the analysis using the matrix element approach.

\begin{tabular}{ccc}
\hline Source & $+\mathrm{SD}$ & $-\mathrm{SD}$ \\
\hline Muon identification & 0.01 & -0.01 \\
Electron identification and smearing & 0.02 & -0.02 \\
PDF & 0.06 & -0.05 \\
Top mass & 0.04 & -0.06 \\
Triggers & 0.02 & -0.02 \\
Opposite charge requirement & 0.01 & -0.01 \\
Jet energy scale & 0.01 & -0.04 \\
Jet reconstruction and identification & 0.02 & -0.06 \\
Background normalisation & 0.07 & -0.08 \\
Monte Carlo statistics & 0.03 & -0.03 \\
Instrumental background & 0.01 & -0.01 \\
Luminosity & 0.04 & -0.04 \\
Other & 0.02 & -0.02 \\
Template statistics & 0.10 & -0.10 \\
\hline Total systematic uncertainty & 0.15 & -0.18 \\
Statistical uncertainty & 0.33 & -0.35 \\
\hline
\end{tabular}




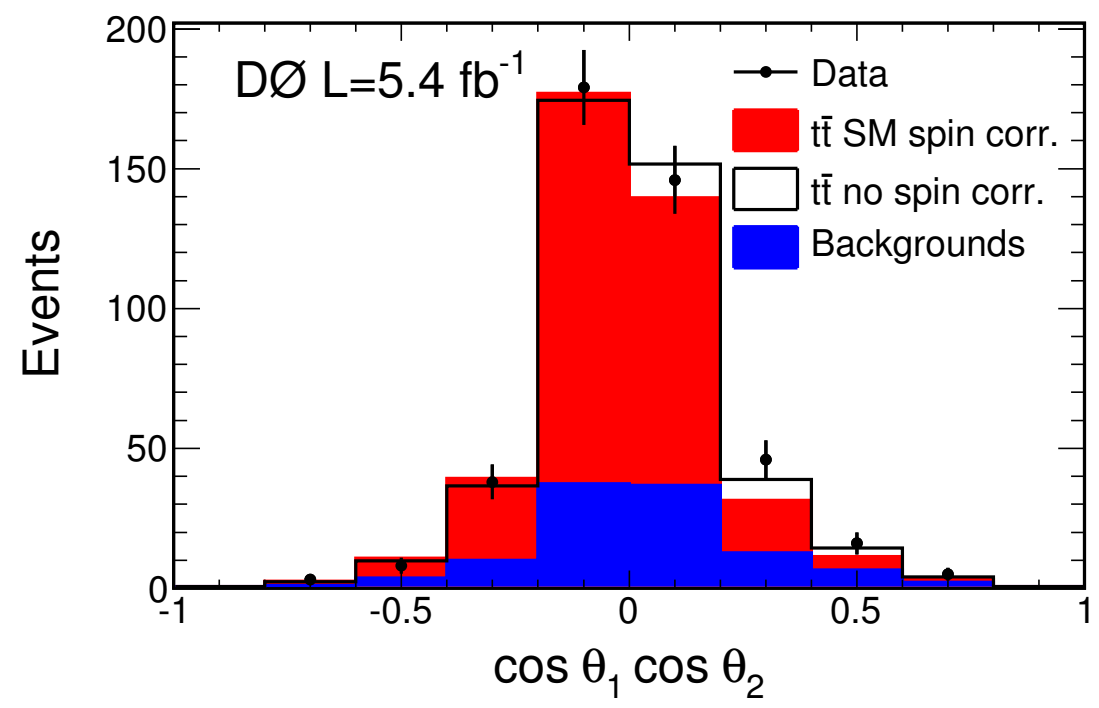

Figure 7.8: The $\cos \theta_{1} \cos \theta_{2}$ distribution for all six analysis channels combined. In blue the sum of all backgrounds, in red the SM t $\bar{t}$ signal. The open histogram shows the case in which t $\bar{t}$ pairs have no spin correlations.

the SM expectation of $C=0.777$ we expect to exclude values below -0.06 at $95 \%$ C.L. From the maximum likelihood fit to data we obtain $C_{\text {meas }}=$ $0.10_{-0.44}^{+0.42}$ (stat+syst), which is shown in Figure 7.9. After transforming $C_{\text {meas }}$ into

$$
C=0.10_{-0.45}^{+0.45}(\text { stat }+ \text { syst }),
$$

we extract the $95 \%$ C.L. band for $C$ as $[-0.66,0.81]$. The result is within two standard deviations of the NLO QCD prediction, but also compatible with the no-correlation hypothesis.

We simultaneously extract the $t \bar{t}$ cross section to be

$$
\sigma_{\mathrm{t} \overline{\mathrm{t}}}=7.92_{-0.93}^{+1.07}(\text { stat }+ \text { syst }) \mathrm{pb}
$$

for $m_{t}=172.5 \mathrm{GeV}$, in agreement with the standard model prediction of $\sigma_{\mathrm{t} \overline{\mathrm{t}}}=7.46_{-0.93}^{+0.48} \mathrm{pb}[64]$.

Using the improved observable based on the matrix element technique described in Section 7.5 the statistical uncertainty is reduced by roughly 


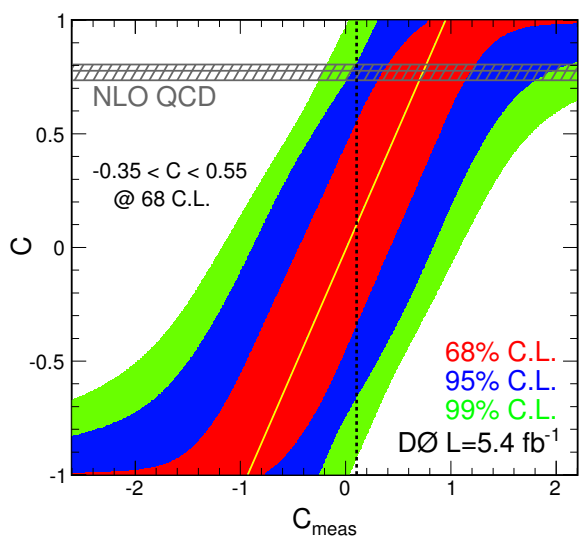

Figure 7.9: Feldman-Cousins bands for the combined fit of all six analysis channels. The $68 \%$ (inner), $95 \%$ (middle), and $99 \%$ (outer) C.L. bands of $C$ as a function of $C_{\text {meas }}$ from the likelihood fits to $\mathrm{MC}$ events for all channels combined. The yellow line indicates the most probable value of $C$ as a function of $C_{\text {meas }}$. The best fit value $C_{\text {meas }}=0.10$ is shown as the dashed black line. The NLO QCD $C=0.777$ prediction is shown as the hashed grey area.

30\%. From the maximum likelihood fit to data we obtain

$$
f_{\text {meas }}=0.74_{-0.41}^{+0.40}(\text { stat }+ \text { syst })
$$

We exclude the no correlation hypothesis $(f=0)$ at the $97.7 \%$ C.L. For the SM value of $f=1$ the no correlation hypothesis is expected to be excluded at $99.6 \%$ C.L., or nearly three standard deviations.

Combining this result with a similar analysis performed in the $\ell+$ jets final state, which is statistically independent of the dilepton selection, we obtain $f_{\text {meas }}=0.85 \pm 0.29$ (stat + syst)which is in good agreement with the SM prediction. For the observed value of $f_{\text {meas }}=0.85$ we can exclude $f<0.052$ at the $99.7 \%$ C.L. This is therefore evidence for SM spin correlations.

This is the first measurement of spin correlations at the D0 experiment during Run II. Recently ATLAS and CMS have published measurements based on the $\Delta \phi$ distribution of the charged leptons. The ATLAS measurement uses $2.1 \mathrm{fb}^{-1}$ of pp collisions and was the first to exclude the no 


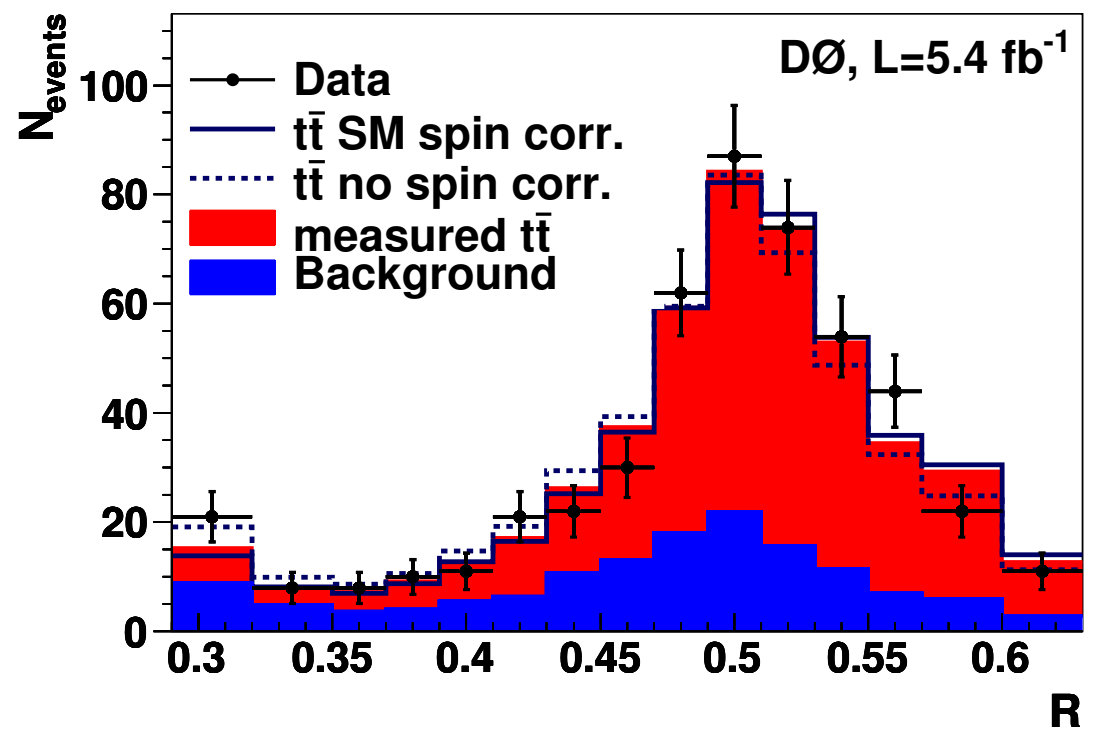

Figure 7.10: The $R$ distribution for all six analysis channels combined. The best fit signal and background contributions are shown in red and blue re-

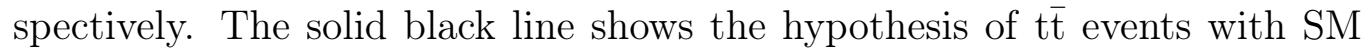
spin correlations. The case of no spin correlation is shown as the dashed black line. 


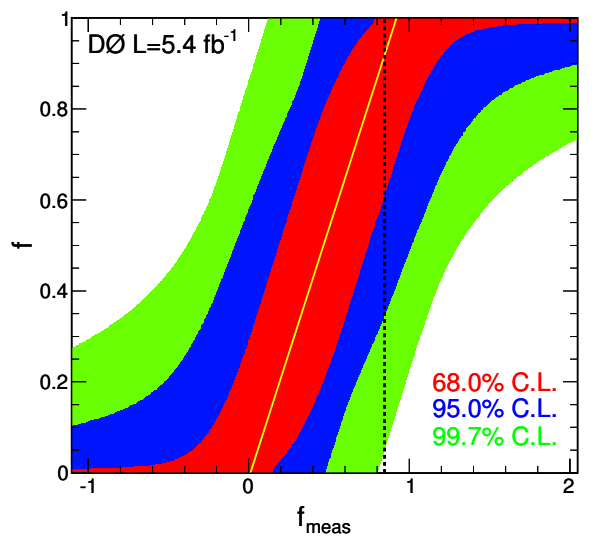

Figure 7.11: Feldman-Cousins bands for the combined fit of all six analysis channels using the matrix element based observable $R$. The $68 \%$ (inner), $95 \%$ (middle), and $99 \%$ (outer) C.L. bands of $f_{\mathrm{SM}}$ as a function of $f_{\text {meas }}$ from the likelihood fits to MC events for all channels combined. The yellow line indicates the most probable value of $f$ as a function of $f_{\text {meas }}$. The best fit value $f_{\text {meas }}=0.74$ is shown as the dashed black line.

spin correlations hypothesis at more than five standard deviations [4]. Using $5 \mathrm{fb}^{-1}$ of pp collisions CMS extract the correlation coefficient $C_{\text {heli }}=$ $0.24 \pm 0.02$ (stat) \pm 0.08 (syst) using the helicity basis.

The t $\overline{\mathrm{t}}$ production mechanisms at the Tevatron collider and LHC are almost exactly opposite, making measurements at both colliders complementary instead of competitive. One of the basic properties of the top quark has gone from being basically unexplored a few years ago, to now being firmly established. Another success for the standard model of particle physics. 


\section{Chapter 8}

\section{Forward Backward Asymmetries}

The measured inclusive t $\overline{\mathrm{t}}$ production cross section is in good agreement with standard model predictions. It is possible that new physics processes modify the inclusive cross section only slightly, but have a large effect on more exclusive observables. The production cross section as a function of scattering angle of the top quark is one such variable. It can be studied using an asymmetry defined as:

$$
A=\frac{N_{F}-N_{B}}{N_{F}+N_{B}}
$$

where $N_{F}$ is the number of forward events and $N_{B}$ is the number of backward events. There are different ways to define forward and backward which will be discussed later.

Long before the discovery of the top quark predictions about an asymmetry in $t \bar{t}$ production were made $[48,65]$. The first publications presenting measurements of the t $\bar{t}$ asymmetry by the CDF [5] and D0 [19] collaborations created a lot of interest as deviations from the SM predictions were observed.

In t $\bar{t}$ production at a $p \bar{p}$ collider the top antiquark is preferentially emitted in the direction of the incoming antiquark. At next-to-leading order in quantum chromodynamics the asymmetry between the number of top quarks emitted in the forward direction (positive $\eta$ ) and backward direction is about

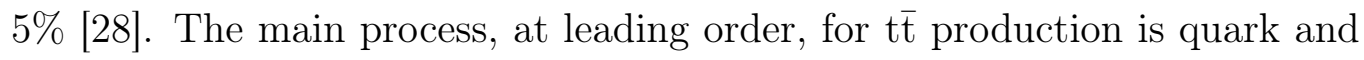
antiquark annihilation to a gluon which then splits into a top quark and 

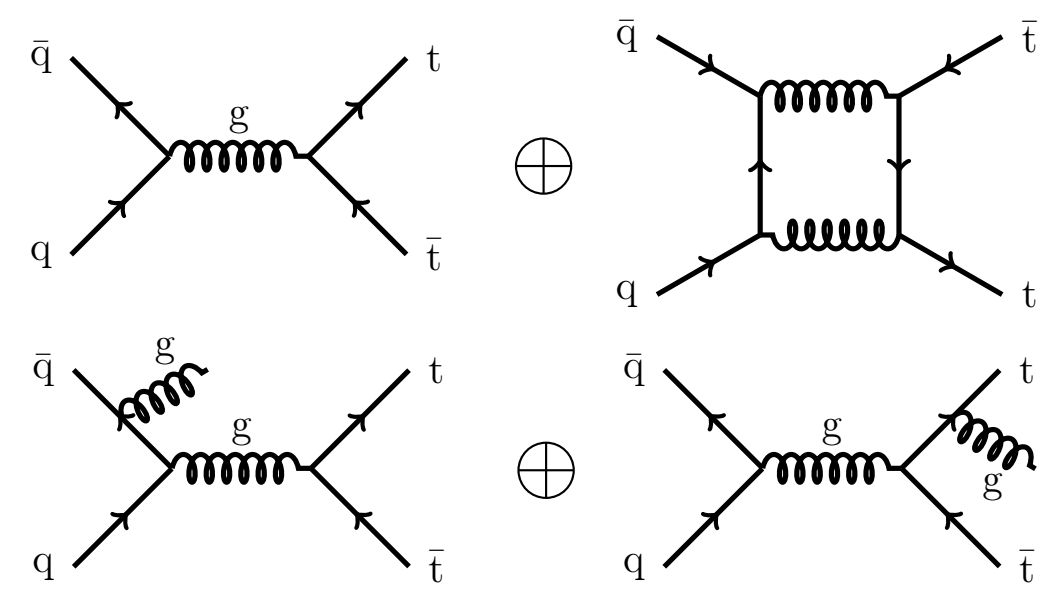

Figure 8.1: Feynman diagrams showing top quark pair production. Diagrams on each row have the same initial and final state, which leads to interference between them. When only the leading order diagram, shown on the top left, is considered the differential cross section with regard to the scattering angle of the top quark is symmetric about zero. The interference of the leading order and next to leading order diagram (top right) gives rise to a positive asymmetry. Similarly the interference between the two diagrams on the bottom leads to an asymmetry.

antiquark pair. The calculation of this process is similar to the calculation of $e^{+} e^{-} \rightarrow \gamma \rightarrow \mu^{+} \mu^{-}$at leading order in QED. The differential cross section is proportional to $1+\cos (\theta)^{2}$, where $\theta$ is the scattering angle between the $\mu^{+}$and $e^{+}$. Although the calculation for $q \bar{q} \rightarrow \mathrm{t} \overline{\mathrm{t}}$ is complicated by the large mass of the top quark and the fact that the mediating photon has been replaced by eight gluons, the process remains symmetric with respect to the scattering angle. No asymmetry arises. At next-to-leading order a positive asymmetry arises, which is driven by the interference between the leading order and next-to-leading order diagrams shown in Figure 8.1.

As a result of the larger than expected values of the top quark asymmetry observed by the CDF and D0 collaborations and a scarcity of other deviations from the standard model in the top sector, theoretical efforts have focussed on constructing models of physics beyond the standard model which could explain the observed top quark asymmetries. New models have to be constructed carefully as they need to satisfy all constraints from a vast body of 

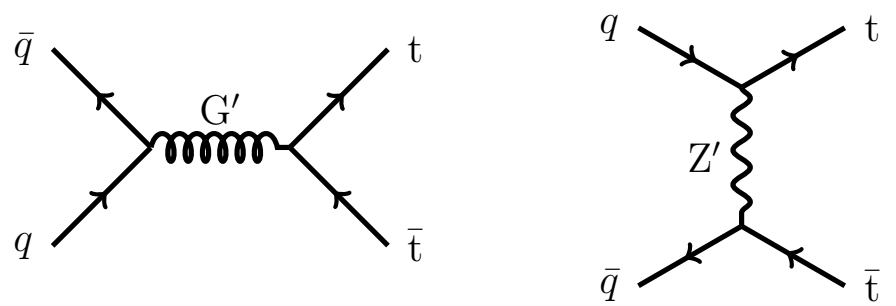

Figure 8.2: Feynman diagrams for $\mathrm{t} \overline{\mathrm{t}}$ production via a massive colour octet (left), and $t$-channel exchange of a Z' boson (right).

experimental results and offer a plausible mechanism which increases the observed asymmetry. For instance, a model predicting an increased asymmetry while simultaneously increasing the total cross section for top quark pair production is of little use as the observed cross section is in good agreement with standard model predictions. Furthermore, precision flavour observables place strong limits on the couplings of the b quark.

One possible avenue to take in order to avoid these constraints is to construct a model which only couples to right handed top quarks. This leads to top quarks with a large polarisation [59]. Models aiming to explain the top quark asymmetry can be divided into two categories: $s$ and $t$-channel. Both have to rely on interference with the standard model diagrams to create an enhanced asymmetry, as creating it purely from beyond the standard model diagrams would lead to an increase of the total cross section. The $s$-channel models propose a new massive, spin 1, colour octet, in essence a massive gluon. In the $t$-channel models the new particle can be either a vector or scalar. If the new particle is electrical neutral, such as a $\mathrm{Z}^{\prime}$ boson, the production of same sign tt pairs is allowed in pp collisions at the LHC. Example diagrams for both types of model are shown in Figure 8.2. Several different scenarios of models, their predicted asymmetry, top quark polarisation and the relation between different observable asymmetries are discussed in references [59].

Previous measurements of the $t \bar{t}$ asymmetry have focussed on the so called $\ell+$ jets final state, in which one of the $\mathrm{W}$ bosons decays to a lepton and one decays hadronically. In this final state the reconstruction of the four 
momenta of the top quark and antiquark are possible. The usual observable chosen in those analyses is the asymmetry between the number of events with $\Delta y=q_{\ell}\left(y_{t, \text { lep }}-y_{t, \text { had }}\right)>0$ and $\Delta y<0$. The rapidity of the leptonically decaying top quark is denoted $y_{t, \text { lep }}$, that of the hadronically decaying top quark as $y_{t, \text { had }}$ and the charge of the lepton is denoted as $q_{\ell}$. More recently a new observable based on $q_{\ell} \cdot \eta_{\ell}$ has been used as well.

In the dilepton final state a full reconstruction of the top quark and antiquark four momenta is challenging but possible. In this analysis no attempt is made to reconstruct the top quark four momenta and instead the charged leptons from the top quark decay are used to construct observables. The resolution of the reconstructed top quark rapidity, $y_{t}$, would be inferior to the excellent resolution with which the rapidity of the charged leptons is measured in the D0 detector. In order to facilitate comparison to theoretical calculations the large resolution of $y_{t}$ would require further correcting for bin-to-bin migrations. These are negligible for the charged leptons. The fact that simple quantities were used as the building blocks of the observables in this analysis should not detract from their importance. Well measured, simple quantities can often provide a lot of physics insight. Especially in the light of the observed deviations from SM predictions in analyses which use observables requiring more complicated reconstruction techniques.

As eluded to in Chapter 7 there exist new physics models which are indistinguishable when considering only the predicted asymmetry. The longitudinal polarisation of the top quark allows for further investigation of possible deviations of the asymmetry from the SM prediction and discriminate between competing explanations. In the standard model top quarks are expected to be produced unpolarised, new physics models predicting larger values of the asymmetry can also lead to non-vanishing longitudinal polarisation of the top quark.

A brief outline of the steps involved in this analysis:

- The event selection is described in Section 6.

- Define observables used.

- Make SM predictions. 
- Compare data and simulation in a background dominated sample.

- Perform background subtraction and correct for the detector acceptance.

- Combine the results of the individual channels.

The observables studied in this analysis are described in the following section.

\subsection{Observables}

Rather than reconstructing the $\mathrm{t}$ and $\overline{\mathrm{t}}$ four momenta, to measure asymmetries related directly to the top quarks. We measure observables based on the pseudo rapidity $\eta$ and electrical charge of the charged leptons.

Related to the asymmetry of the number of top quarks and antiquarks produced in the forward direction $(\eta>0)$ is the leptonic charge asymmetry:

$$
A^{\ell}=\frac{N_{\ell^{+}}(\eta>0)-N_{\ell^{-}}(\eta>0)}{N_{\ell^{+}}(\eta>0)+N_{\ell^{-}}(\eta>0)}
$$

here $N_{\ell^{+}}(\eta)\left(N_{\ell^{-}}(\eta)\right)$ is the number of leptons with positive (negative) electric charge as a function of $\eta$.

If CP invariance holds, then $N_{\ell^{+}}(\eta)=N_{\ell^{-}}(-\eta)$, the number of antileptons as a function of $\eta$ is equal to the number of leptons as a function of $-\eta$. As a result $A^{\ell}=A_{\mathrm{FB}}^{\ell^{+}}=-A_{\mathrm{FB}}^{\ell^{-}}$, where

$$
A_{\mathrm{FB}}^{\ell^{ \pm}}=\frac{N_{\ell^{ \pm}}(\eta>0)-N_{\ell^{ \pm}}(\eta<0)}{N_{\ell^{ \pm}}(\eta>0)+N_{\ell^{ \pm}}(\eta<0)} .
$$

The asymmetry $A_{\mathrm{FB}}^{\ell^{+}}$uses only leptons with positive electrical charge and $A_{\mathrm{FB}}^{\ell^{-}}$uses only leptons with negative electrical charge. This means these two numbers are statistically independent. The two asymmetries are expected to be of equal magnitude but opposite sign if CP is conserved. After checking that there is no deviation from this expectation the two samples can be combined in:

$$
A_{\mathrm{FB}}^{\ell}=\frac{N_{\ell}(Q \cdot \eta>0)-N_{\ell}(Q \cdot \eta<0)}{N_{\ell}(Q \cdot \eta>0)+N_{\ell}(Q \cdot \eta<0)}
$$


where $Q$ is the charge of the lepton. Both leptons from each event are used, which reduces the statistical uncertainty by $\sqrt{2}$ compared to $A_{\mathrm{FB}}^{\ell^{ \pm}}$. This asymmetry is also measured in the most recent D0 analysis in the $\ell+$ jets final state.

In analogy to the FB asymmetry for top quarks and antiquarks, we define an angular asymmetry for leptons:

$$
A^{\ell \ell}=\frac{N(\Delta \eta>0)-N(\Delta \eta<0)}{N(\Delta \eta>0)+N(\Delta \eta<0)}
$$

where $\Delta \eta=\eta_{\ell^{+}}-\eta_{\ell^{-}}$. The asymmetry $A_{\mathrm{CP}}^{\ell}$ corresponds to a longitudinal asymmetry in spin orientation relative to the proton beam direction. It is defined as:

$$
A_{\mathrm{CP}}^{\ell}=\frac{N_{\ell^{+}}(\eta>0)-N_{\ell^{-}}(\eta<0)}{N_{\ell^{+}}(\eta>0)+N_{\ell^{-}}(\eta<0)} .
$$

This asymmetry is sensitive to $s$-channel exchanges of heavy non-scalar resonances with $\mathrm{CP}$-violating couplings to quarks, but not to possible $\mathrm{P}$ and CP-violating effects from an $s$-channel exchange of Higgs bosons.

All these asymmetries are based on the pseudo-rapidity distributions of the charged leptons. All the quantities needed to derive the asymmetries considered here are contained in the following four distributions:

- pseudo-rapidity distribution of leptons with positive electrical charge, $\eta_{\ell^{+}}$

- pseudo-rapidity distribution of leptons with negative electrical charge, $\eta_{\ell^{-}}$

- charge signed pseudo-rapidity distribution, leptons of both electrical charges are combined, $Q \cdot \eta$; and

- delta pseudo-rapidity distribution, the difference between the rapidity of the antilepton and lepton, $\eta_{\ell^{+}}-\eta_{\ell^{-}}$.

In Figures 8.3 and 8.4 these four distributions are shown summed over all final states and run periods. 


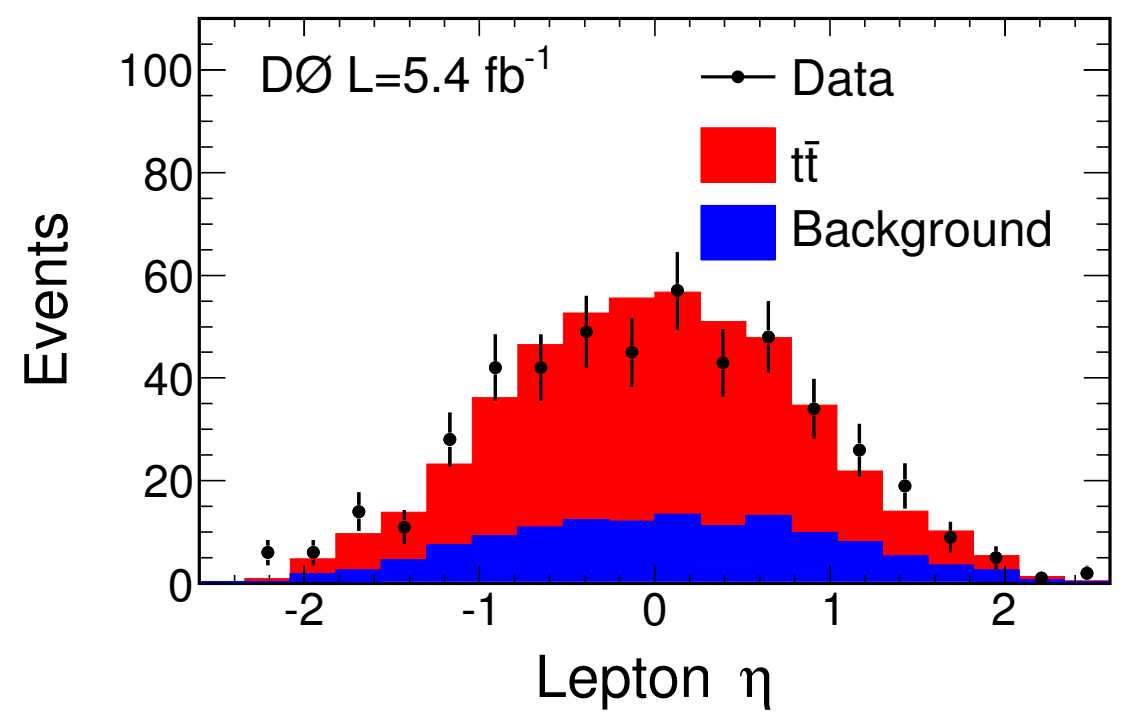

(a)

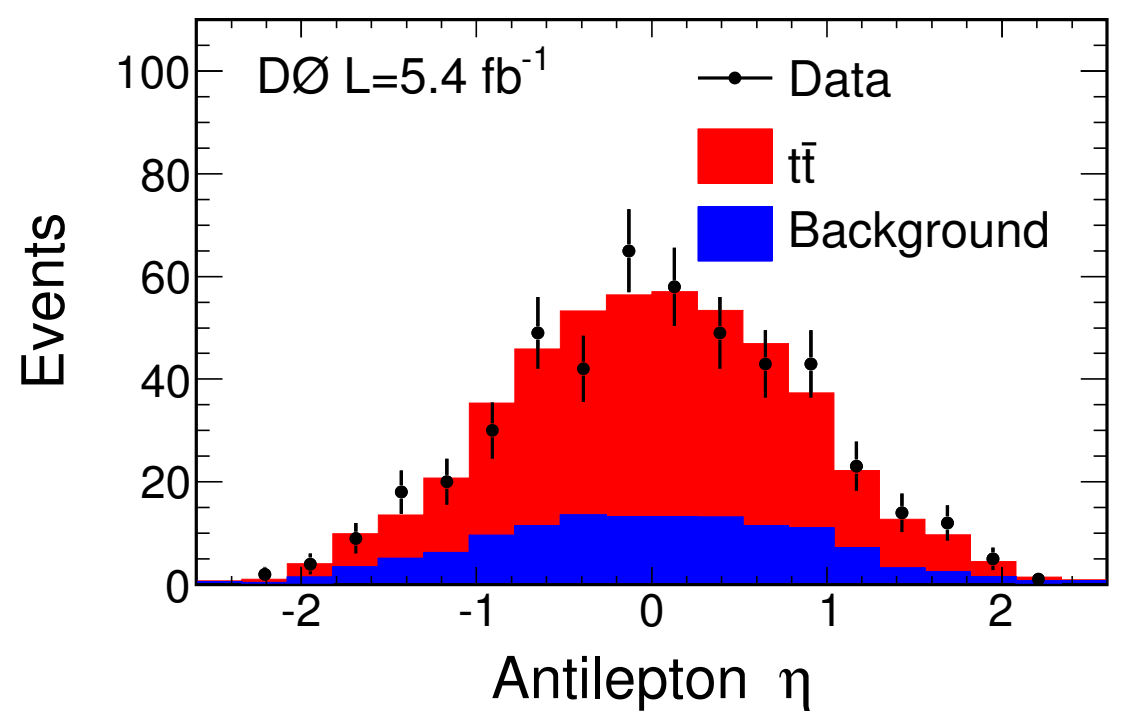

(b)

Figure 8.3: The lepton rapidity distributions used in the calculation of the asymmetries: $A^{\ell}, A_{\mathrm{FB}}^{\ell^{ \pm}}$and $A_{\mathrm{CP}}^{\ell}$. The pseudo-rapidity of the negatively and positively charged lepton are shown in (a) and (b) respectively. In all plots the final selection cuts have been applied. The sum of all three final states and both run periods is shown, with the tét contribution normalised to a cross section of $8.05 \mathrm{pb}^{-1}[11]$. 


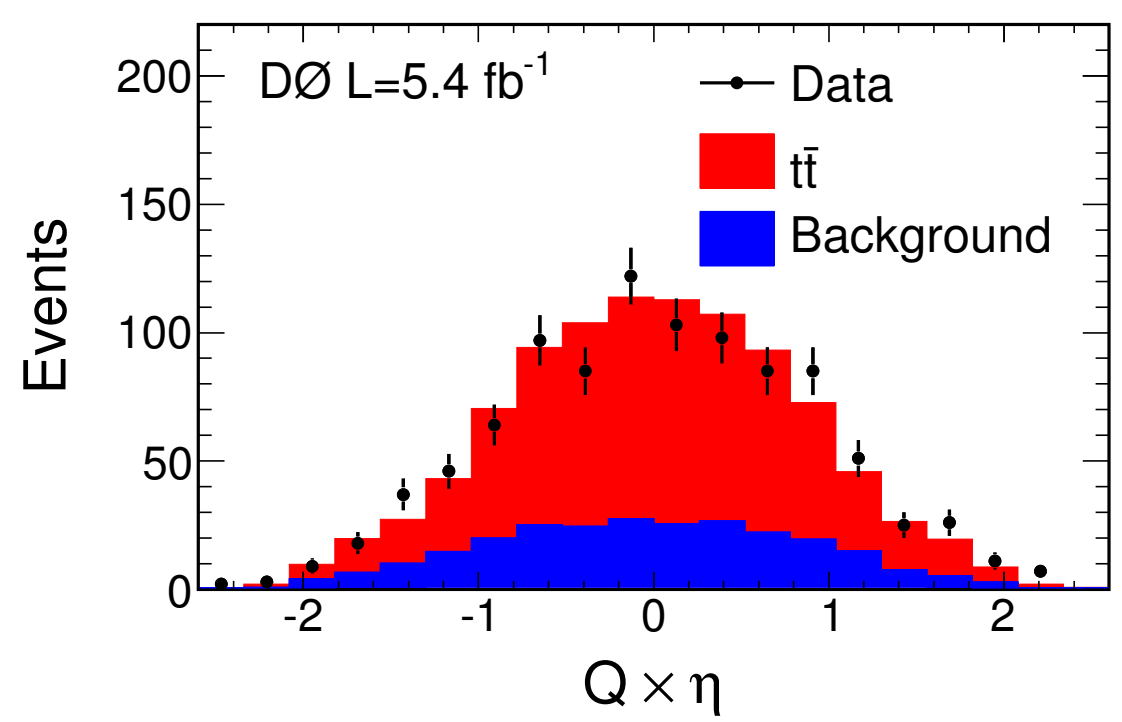

(a)

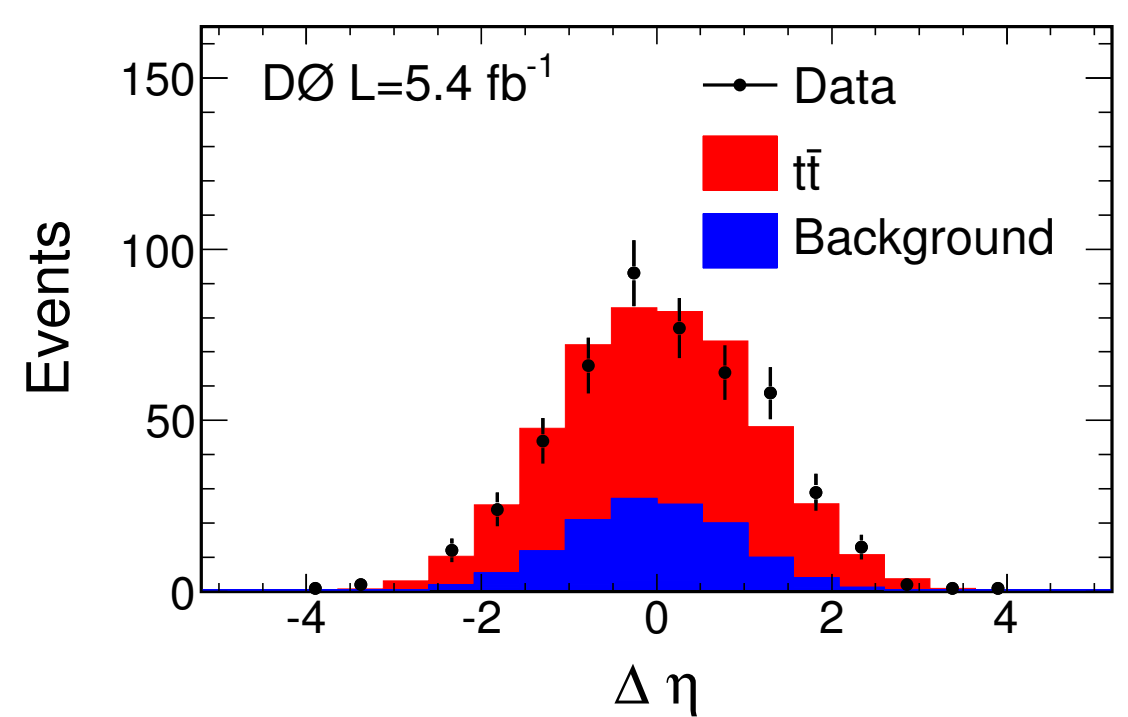

(b)

Figure 8.4: The lepton rapidity distributions used in the calculation of the asymmetries $A^{\ell \ell}$ and $A_{\mathrm{FB}}^{\ell}$. In (a) the leptons of both charges are combined in the $Q \times \eta$ distribution. The distribution of $\Delta \eta=\eta_{\ell^{+}}-\eta_{\ell^{-}}$is shown in (b). In both plots the final selection cuts have been applied. The sum of all three final states and both run periods is shown, with the t $\bar{t}$ contribution normalised to a cross section of $8.05 \mathrm{pb}^{-1}$ [11]. 


\subsection{Predictions}

A simulation of each of the background processes and the tit signal process are used to make predictions for each one of the asymmetries. After the events are processed by the GEANT simulation they are reconstructed by the same software as used for the real data. The same selection criteria as for the data are then applied and the asymmetries are calculated in the same way as in data. For example the number of events with $\Delta \eta<0$ and $\Delta \eta>0$ are calculated and then used to predict $A^{\ell \ell}$.

While the simulation of t $\overline{\mathrm{t}}$ events in MC@NLO includes diagrams up to next-to-leading order for the production of top quark pairs, their decay is simulated at LO only. In theoretical calculations both production and decay are computed at next-to-leading order. A further difference between the predicted asymmetry taken from a simulation like MC@NLO and a theoretical calculation arises from the following. Two different approaches can be taken to calculate an asymmetry

$$
A=\frac{\sigma^{F}-\sigma^{B}}{\sigma^{F}+\sigma^{B}}=\frac{\Delta \sigma}{\sigma}
$$

where $\sigma^{F}$ and $\sigma^{B}$ are the integrated cross sections for the forward and backward direction. The first, used in programs like MC@NLO is to calculate $\sigma^{F}$ and $\sigma^{B}$ independently by integrating the differential cross section $\frac{\partial \sigma}{\partial x}$

$$
\sigma^{B}=\int_{-\infty}^{0} \frac{\partial \sigma}{\partial x} \mathrm{~d} x \text { and } \sigma^{F}=\int_{0}^{+\infty} \frac{\partial \sigma}{\partial x} \mathrm{~d} x
$$

where $x$ is the variable used to define forwards and backwards. Most theoretical calculations [23] make use of the fact that the leading order terms for $\sigma^{B}$ and $\sigma^{F}$ cancel in $\Delta \sigma$. The asymmetry is expanded in orders of $\alpha_{s}$ :

$$
A=\alpha_{s}\left(\frac{\Delta \sigma_{\mathrm{NLO}}+\alpha_{s} \Delta \sigma_{\mathrm{NNLO}}+\ldots}{\sigma_{\mathrm{LO}}+\alpha_{s} \delta\left(\sigma_{\mathrm{NLO}}\right)+\ldots}\right)
$$

where $\Delta \sigma_{\mathrm{k}}$ is the difference between $\sigma^{F}$ and $\sigma^{B}$ at order $k$ and $\delta\left(\sigma_{\mathrm{NLO}}\right)$ are the NLO terms in the cross section expansion. The result of this choice of 
expansion is that the denominator is the leading order cross section.

In this analysis the choice was made to use the theoretical prediction from Reference [29] and reweight the events from MC@NLO. We apply the reweighting simultaneously as a function of the lepton and antilepton rapidity. In Figures 8.5 and 8.6 the lepton rapidity distributions before and after reweighting are shown.

\subsection{Background cross check}

The dimuon and dielectron channel present an ideal opportunity to validate the analysis in a high statistics sample. At the stage of the basic selection the events originate mainly from $\mathrm{Z}+$ jets production. The only difference to the final analysis sample is that the topological cuts $\left(\sigma_{\mathbb{E}_{T}}\right.$ and $\left.\mathbb{E}_{T}\right)$ have not been applied. These samples are ideally suited to test the analysis procedure as they have high statistics and the charged leptons in $Z+$ jets production are expected to have an angular asymmetry.

The same procedure as for the actual measurement is used to compare the predicted asymmetry with the one measured in data. The only difference is that we treat $\mathrm{Z}+$ jets production as the signal instead of a background process. The four distributions used to derive the asymmetries are shown in Figures 8.7 and 8.8 .

For each of the six asymmetries we give the value of the asymmetry measured in data, which we compare to the prediction from simulation. After subtracting the backgrounds from the data we compare the observed value of the asymmetries to a prediction made using only $\mathrm{Z}+$ jets events.

By comparing the values of $A_{\mathrm{FB}}^{\ell^{+}}$and $A_{\mathrm{FB}}^{\ell^{-}}$we can check whether CP invariance holds, or alternatively if the detector favours leptons of one particular charge. We find that the sum of these two asymmetries is compatible with zero. Generally there is good agreement between the predictions and observed values in data, both before and after subtracting the backgrounds. The results are given in Table 8.1. 


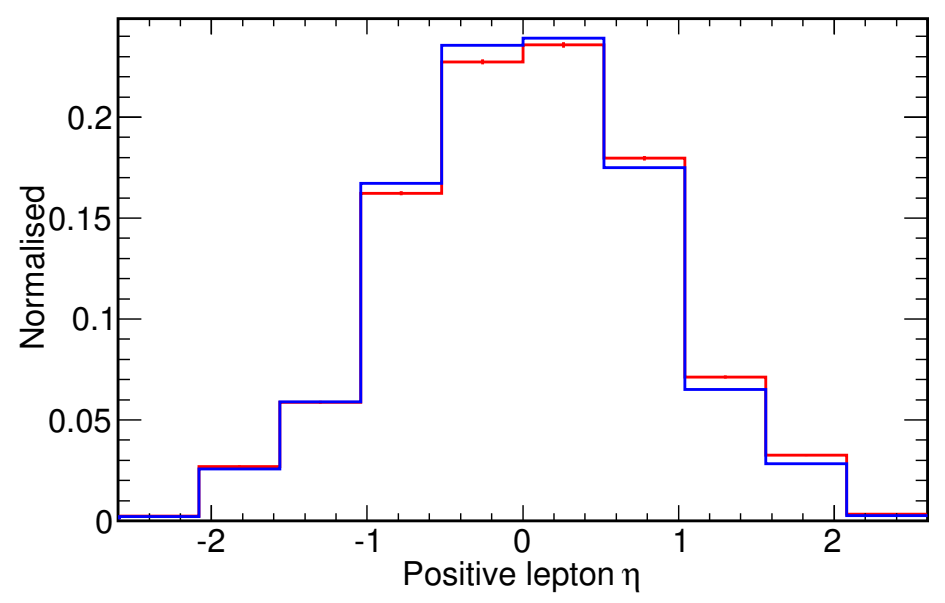

(a)

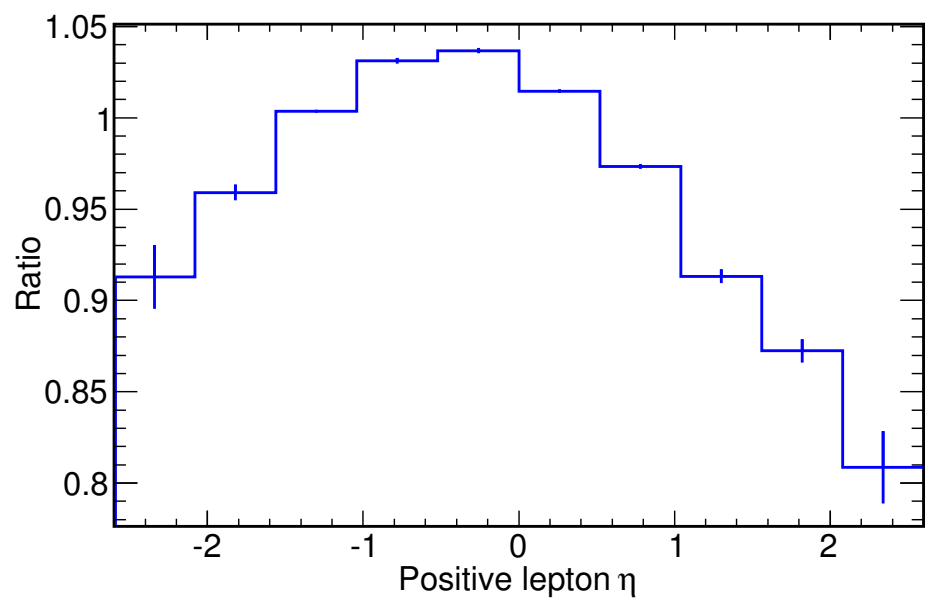

(b)

Figure 8.5: The rapidity distribution of leptons with positive electric charge is shown in (a). The asymmetry is increased by reweighting to the theoretical calculation in Reference [29]. The blue distribution shows MC@NLO out of the box and the red distribution shows the result of the reweighting. Below (b) shows the ratio of the two. 


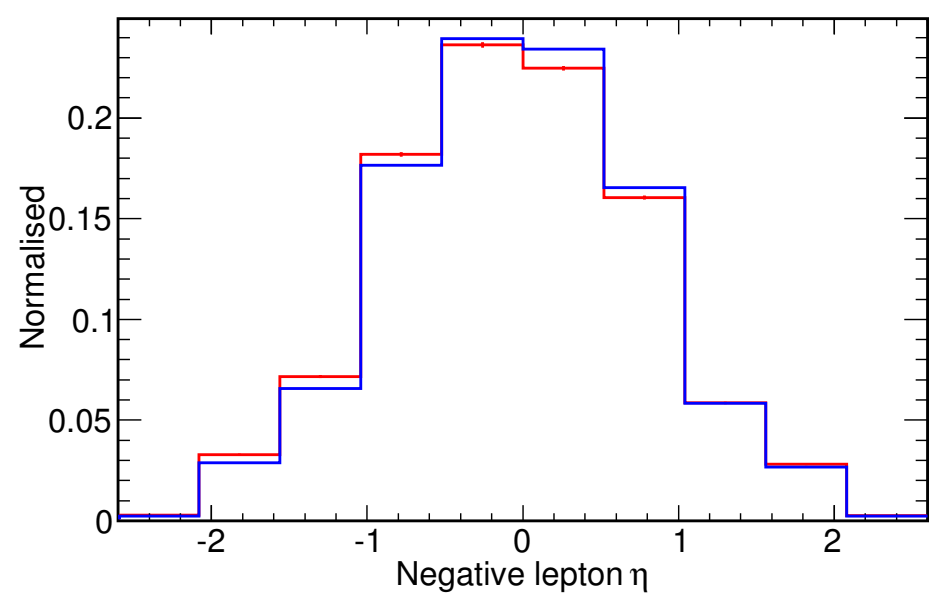

(a)

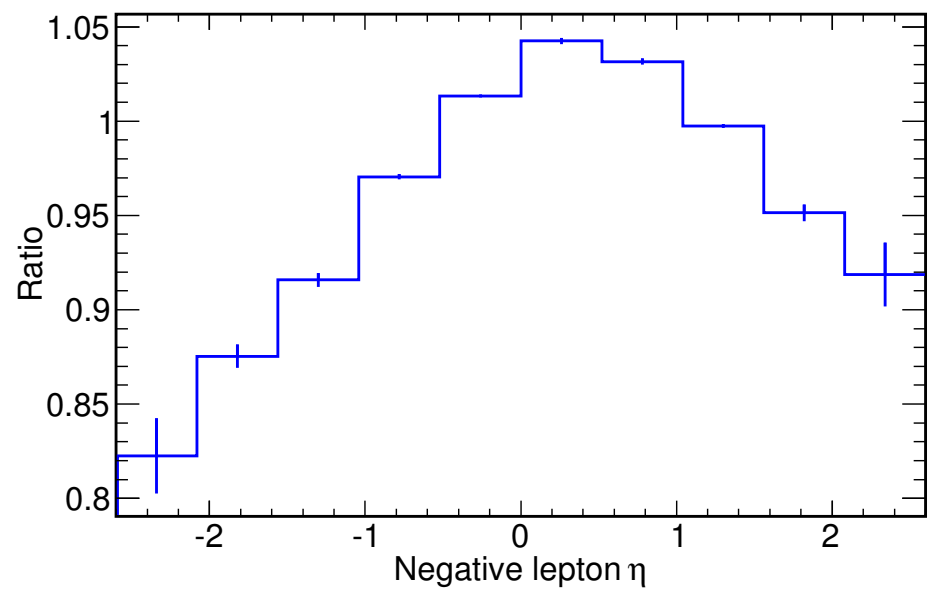

(b)

Figure 8.6: The rapidity distribution of leptons with negative electric charge is shown in (a). The asymmetry is increased by reweighting to the theoretical calculation in Reference [29]. The blue distribution shows MC@NLO out of the box and the red distribution shows the result of the reweighting. Below (b) shows the ratio of the two. 


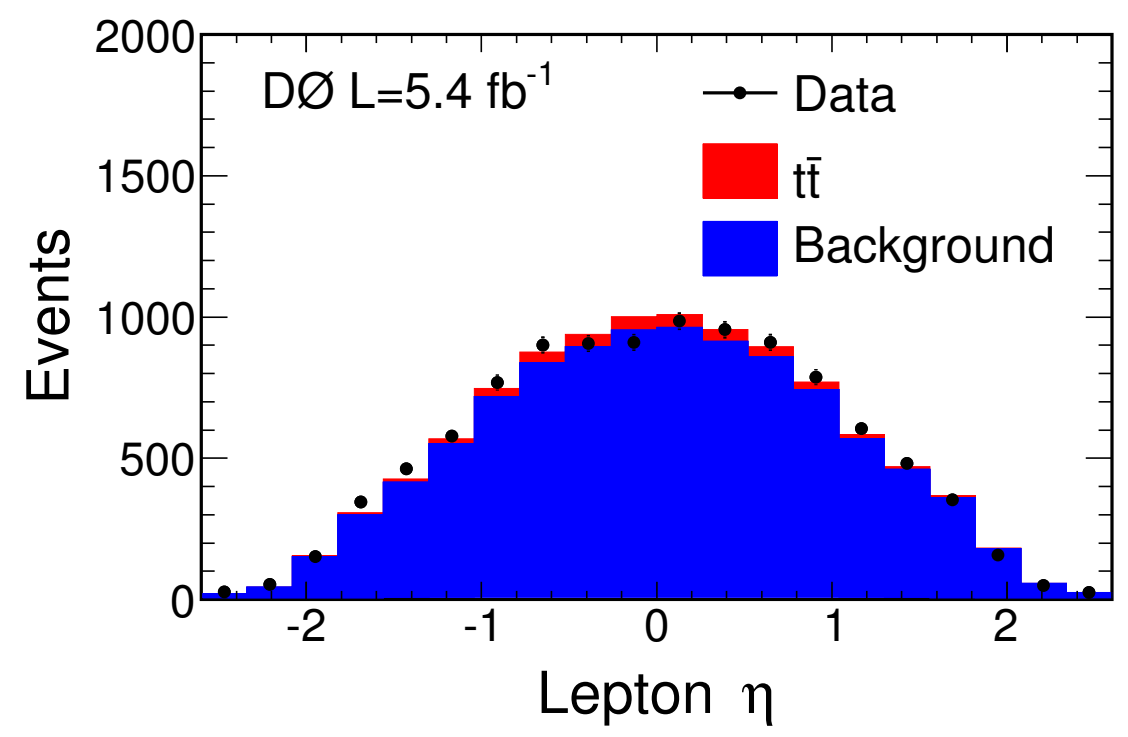

(a)

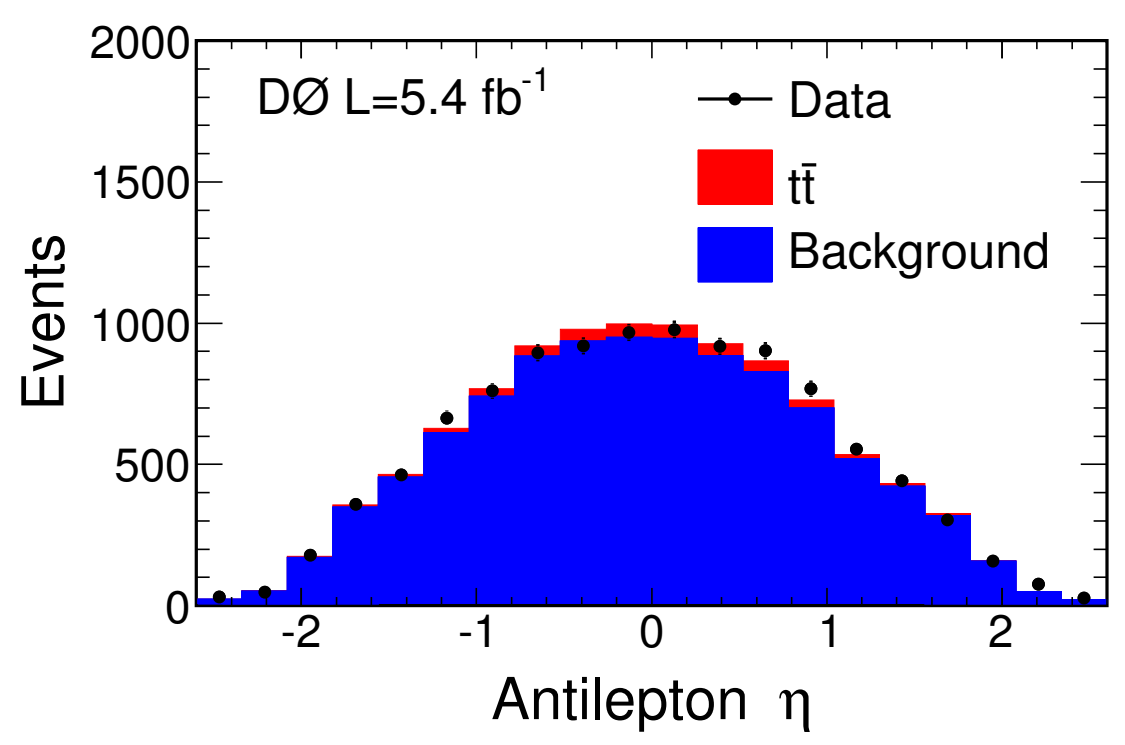

(b)

Figure 8.7: The lepton rapidity distributions used in the calculation of the asymmetries: $A^{\ell}, A_{\mathrm{FB}}^{\ell^{ \pm}}$and $A_{\mathrm{CP}}^{\ell}$. The pseudo-rapidity of the negatively and positively charged lepton are shown in (a) and (b) respectively. The final selection cuts have not been applied and as a result the majority of events are from $Z+$ jet production. The sum of the dimuon and dielectron final states over both run periods is shown. 


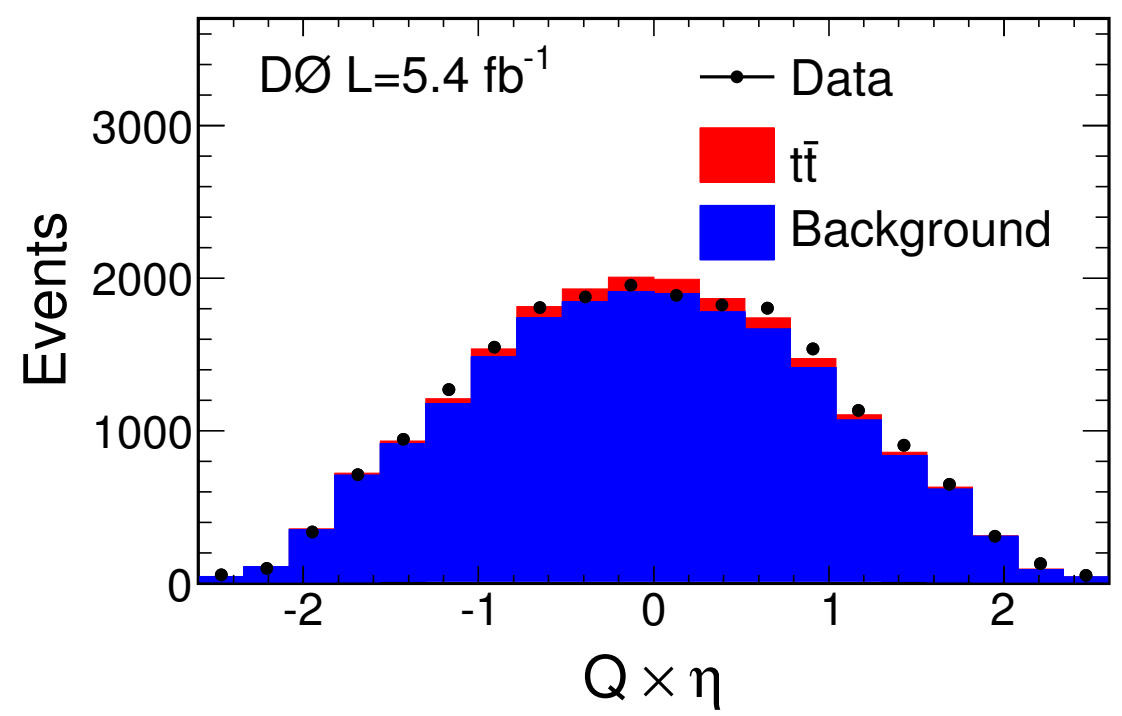

(a)

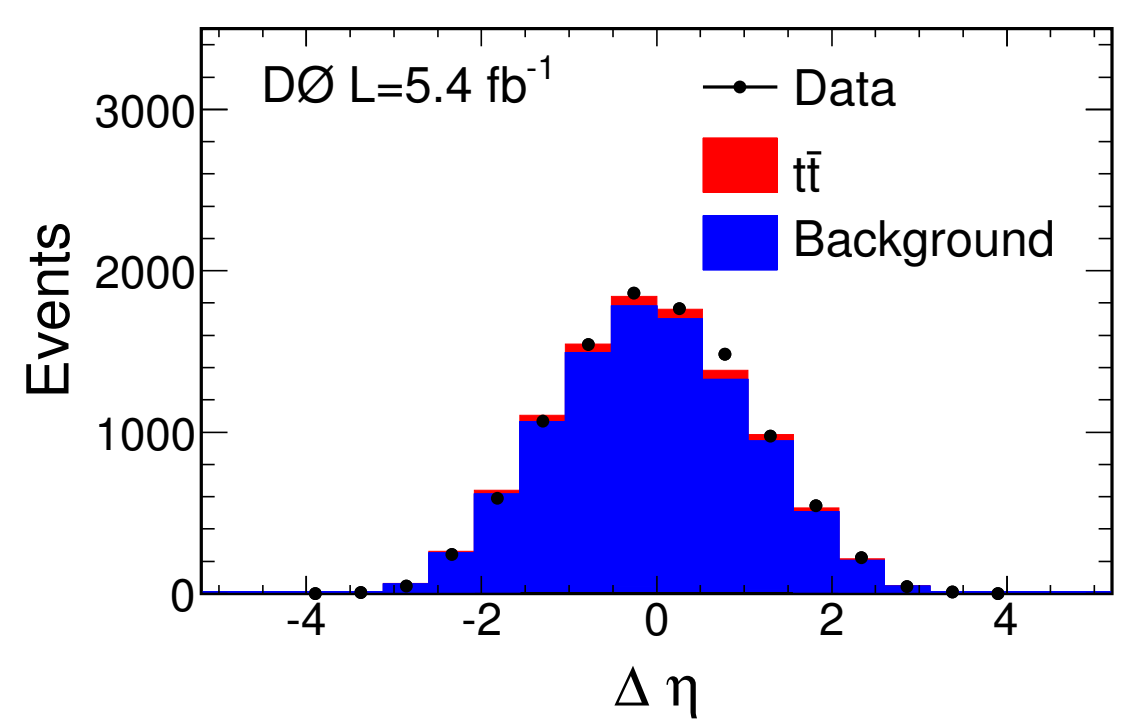

(b)

Figure 8.8: The lepton rapidity distributions used in the calculation of the asymmetries $A^{\ell \ell}$ and $A_{\mathrm{FB}}^{\ell}$. In (a) the leptons of both charges are combined in the $Q \times \eta$ distribution. The distribution of $\Delta \eta=\eta_{\ell^{+}}-\eta_{\ell^{-}}$is shown in (b). The final selection cuts have not been applied and as a result the majority of events are from $Z+$ jet production. The sum of the dimuon and dielectron final states over both run periods is shown. 
Table 8.1: Using the high statistics samples of $Z+$ jets events in the basic selection the six asymmetries are measured. The raw value is obtained by counting events in data, which is compared to the predicted value using simulation. The signal only simulation is compared to the value subtracting the backgrounds.

\begin{tabular}{rrrrc}
\hline & Raw $(\%)$ & Predicted $(\%)$ & Bkg. subtracted $(\%)$ & Signal $(\%)$ \\
\hline$A^{\ell}$ & $-1.8 \pm 1.0$ & $-2.8 \pm 0.3$ & $-1.9 \pm 1.0$ & $-2.9 \pm 0.3$ \\
$A_{\mathrm{FB}}^{\ell^{-}}$ & $1.9 \pm 1.0$ & $2.2 \pm 0.3$ & $2.1 \pm 1.0$ & $2.4 \pm 0.3$ \\
$A_{\mathrm{FB}}^{\ell^{+}}$ & $-1.7 \pm 1.0$ & $-3.3 \pm 0.3$ & $-1.8 \pm 1.0$ & $-3.4 \pm 0.3$ \\
$A_{\mathrm{FB}}^{\ell}$ & $-1.8 \pm 0.7$ & $-2.8 \pm 0.2$ & $-1.9 \pm 0.7$ & $-2.9 \pm 0.2$ \\
$A_{\mathrm{CP}}^{\ell}$ & $0.1 \pm 1.0$ & $-0.5 \pm 0.3$ & $0.1 \pm 1.1$ & $-0.5 \pm 0.3$ \\
$A^{\ell \ell}$ & $-3.2 \pm 1.0$ & $-5.0 \pm 0.3$ & $-3.3 \pm 1.1$ & $-5.3 \pm 0.3$ \\
\hline
\end{tabular}

\subsection{Acceptance correction}

Experimental observations are made using a detector of some kind. Rarely can the observations directly be compared to predictions made by a theoretical calculation. Almost always will the detector used to make the observations effect what is observed. These effects can take many shapes, from dead time between observations, to inefficiencies due to a damaged detector, to inactive regions of the detector due to support structures, and aberration in optics. A complex detector system such as the D0 detector necessarily suffers from many such effects.

The GEANT simulation of the D0 experiment is the primary tool for estimating the effects that the detector and reconstruction algorithms have on the underlying physics. Generally the approach is to take a theoretical calculation, and use the GEANT model to make predictions that can be compared directly to the experimental data. This way of comparing theory and experiment becomes clumsy because it needs to be repeated every time a new theoretical model is created. For each one the calculation has to be fed through the GEANT model and the output compared to data.

While theoretical models change and the number of them is infinite, there is only one set of experimental data. An attractive alternative is then to take the experimental observations and undo the effects of the detection equip- 
ment. This is referred to as unfolding. Once the data have been unfolded, they can be directly compared to any theoretical calculations.

The influence a detector has on observables can be expressed as:

$$
\vec{N}_{\text {obs }}=\mathbf{S A} \vec{N}_{\text {theory }}
$$

where $\vec{N}_{\text {obs }}$ represents a vector of $n$ observations which a calculation predicts to be $\vec{N}_{\text {theory }}$. In this analysis the vectors represent the $n$ bins of the histogram in the variable used to calculate the forward backward asymmetry, for example $\Delta \eta$. The $n \times n$ matrices $\mathbf{A}$ and $\mathbf{S}$ are the acceptance and migration matrix. The matrix $\mathbf{S}$ gives the probability of an event which is in bin $i$ of $\vec{N}_{\text {theory }}$ to be observed in a different bin $j$ of $\vec{N}_{\text {obs }}$. The acceptance matrix A is a diagonal matrix of the probability that an event in bin $i$ of $\vec{N}_{\text {theory }}$ is detected by the experiment. This matrix encapsulates the fact that different regions of the detector have different efficiencies, the extreme example of this being a region where the detector does not work.

The charged lepton rapidities are constructed purely from measurements of angles. These are measured with very high precision in the D0 detector, which makes migrations between bins negligible. As a result only acceptance effects need to be corrected for in order to undo the detector effects, by approximating the matrix $\mathbf{S}$ as the identity matrix $\mathbf{I}$ the relation between $\vec{N}_{\text {obs }}$ and $\vec{N}_{\text {theory }}$ becomes:

$$
\vec{N}_{\text {obs }}=\mathbf{A} \vec{N}_{\text {theory }}
$$

Unfolding for detector effects then becomes: $\vec{N}_{\text {theory }}=\mathbf{A}^{-1} \vec{N}_{\text {obs }}$, or expressed in terms of individual bins of a histogram:

$$
n_{i}^{\text {theory }}=\frac{1}{\epsilon_{i}} n_{i}^{\text {obs }}
$$

where $\epsilon_{i}$ is the probability that an event in bin $i$ of $\vec{N}_{\text {theory }}$ is observed by the experiment.

The small contributions to the final sample composition made by back- 
ground process is subtracted from the number of observed events. The number of events after unfolding for acceptance effects is then calculated using selection probabilities $\epsilon_{i}$ derived from simulated $t \bar{t}$ events.

The GEANT simulation of the D0 detector is not perfect, a difference between the simulated acceptance and the true acceptance could lead to a deviation of the observed from the predicted asymmetry. For example if the acceptance for negatively charged leptons in the simulation is uniform as a function of lepton rapidity, but in reality one hemisphere of the detector was not operational for half the time during which data was taken. Assuming, for simplicity, a process which has no asymmetry $A_{\mathrm{FB}}^{\ell^{-}}$, a non zero asymmetry would be observed in data. After applying the acceptance correction derived from the simulation, this asymmetry would persist. The experimenter would incorrectly conclude to have measured a deviation from the predictions. However, an observable like $A_{\mathrm{FB}}^{\ell}$ which combines leptons of both charges would be unaffected by such a major mismodelling. In order to generate an asymmetry in $A_{\mathrm{FB}}^{\ell}$ inefficiencies in a hemisphere would have to depend on the charge of the lepton.

Most effects which could generate such asymmetries between hemispheres or lepton charges are removed by reversing the direction of the magnetic fields in the D0 detector. The polarities of both the solenoid and toroid are regularly reversed leading to nearly equal sized datasets in each of the four possible configurations.

As it is not possible to measure the full acceptance correction needed for the unfolding procedure in data as it includes not just the lepton acceptance but also that for the jets and other selection requirements, we derive a systematic uncertainty to cover potential mismodelling of the acceptance taken from simulation. The charged lepton acceptance is measured using $\mathrm{Z} \rightarrow \ell \ell$ events. The large production cross section and distinctive signature makes it possible to select decays of the $\mathrm{Z}$ boson to two leptons while placing only loose requirements on the second lepton. The lepton fulfilling only loose selection and quality criteria can then be used to study the lepton acceptance.

In the dielectron channel the events are selected which have one lepton which fulfils the same criteria as in the asymmetry analysis and one electron 
which only satisfies loose selection criteria. Similarly for the dimuon channel we require one muon to be of the same quality as in the analysis and one passing only loose requirements. Furthermore the invariant mass of the two leptons must fall within the range of $80 \mathrm{GeV}$ to $100 \mathrm{GeV}$. If the loose lepton fulfils the quality criteria of the lepton selection used in the asymmetry analysis it is counted as passing the acceptance cuts. The systematic uncertainty is derived from the difference between the acceptance at $-\eta$ and $\eta$.

The observed asymmetries after subtracting the backgrounds are shown for each channel in Table 8.2. Predictions made from t $\bar{t}$ simulation and the observed asymmetries after correcting for the acceptance are shown as well.

\subsection{Top polarisation}

As a result of the deviations from the standard model predictions observed in the D0 and CDF measurements performed in the $\ell+$ jets final state, several theoretical models which aim to explain the deviation have been proposed. Some of these also propose that the top quarks are produced with a net polarisation [59]. This is in contrast to the SM, where top quarks are produced with no net polarisation.

An observable sensitive to top quark polarisation can therefore be used to disentangle models of new physics which predict the same value for the top quark charge asymmetry. One such observable is the angle between the direction of the charged lepton and the direction of the top quark in the t $\bar{t}$ rest frame.

This is the angle $\theta_{1,2}$ reconstructed during the measurement of spin correlations. The only difference being the choice of reference direction. Instead of choosing the direction of the beam, the direction of the top quark is used. The method to reconstruct the angle is exactly as described in Section 7.2.

In the absence of detector and kinematical acceptance effects the distribution of $\cos \theta_{1}$ for the lepton, and $\cos \theta_{2}$ for the antilepton, should be isotropic. A longitudinal polarisation of the top quark would cause an asymmetric $\cos \theta_{1,2}$ distribution.

In Figure 8.9 the sum of the $\cos \theta_{1}$ and $\cos \theta_{2}$ distributions is shown. As 


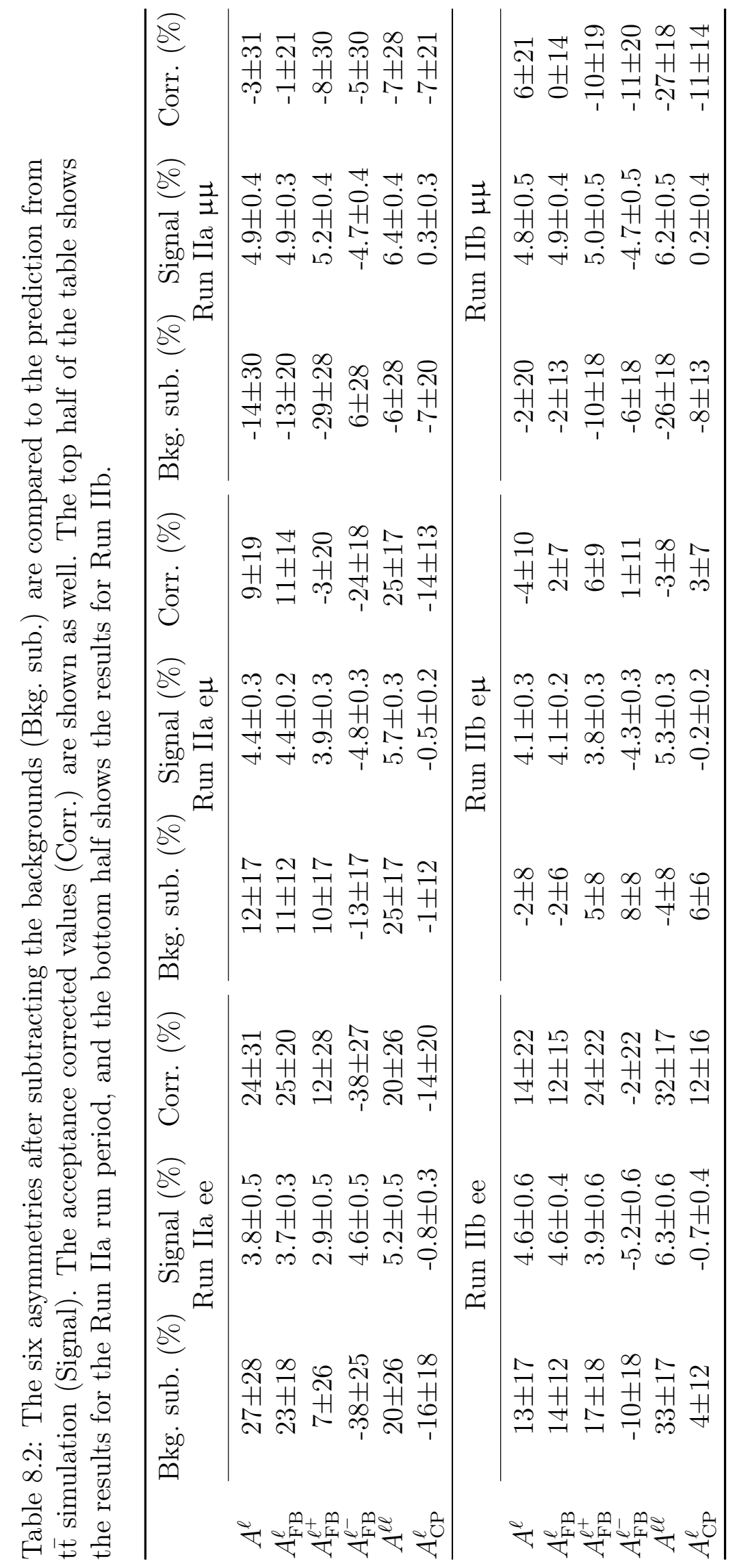




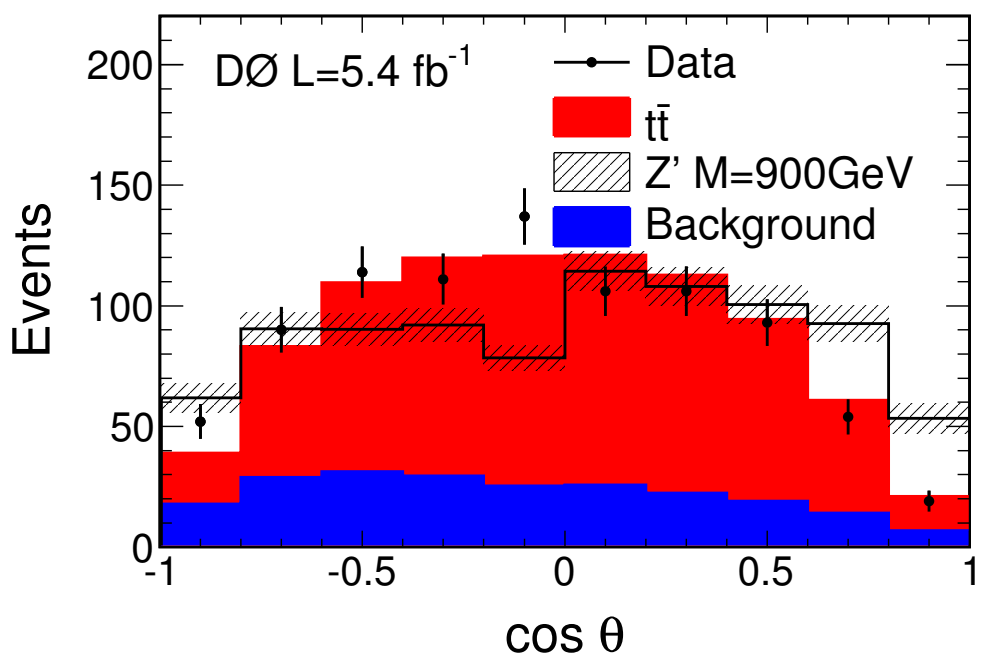

Figure 8.9: The distribution of $\cos \theta$ is shown for the combination of the three dilepton channels. The data are compared to the SM predictions. The distribution of $t \bar{t}$ pairs produced via a hypothetical $Z^{\prime}$ boson is also shown; the uncertainty due to the limited size of the MC sample is shown by the shaded band.

an example of a model containing parity violating couplings a leptophobic topcolour $\mathrm{Z}^{\prime}$ boson is used. It has the same parity-violating couplings to quarks as the standard model $\mathrm{Z}$ boson and a width of $\Gamma=0.012 M_{Z}[15,50]$.

The agreement between the data and the standard model prediction in the distribution is good, yielding a Kolmogorov-Smirnov test probability of 14\%. Observables sensitive to top quark polarisation provide a powerful tool to distinguish different models of new physics which predict similar values of the top quark charge asymmetry.

\subsection{Combination}

To increase the sensitivity of the measurement the individual channels are analysed separately. The final step of the analysis is then to combine them to produce the final result. The standard method for combining different experimental results is to weight each result $x_{i}$ by the inverse of its variance 
$\sigma_{i}$. However, the weighted mean

$$
\hat{x}=\frac{\sum x_{i} / \sigma_{i}^{2}}{\sum 1 / \sigma_{i}^{2}}
$$

and its uncertainty

$$
\frac{1}{\sigma^{2}}=\sum \frac{1}{\sigma_{i}^{2}}
$$

apply only when the uncertainties of the individual measurements are uncorrelated. While the individual channels are statistically independent, some of the systematic uncertainties are correlated between them.

To take these correlations into account we use a technique named: best linear unbiassed estimate (BLUE) to perform the combination [60]. The BLUE method makes use of the full error matrix $\mathbf{E}$, the diagonal elements of which are the variances of the individual measurements $x_{i}$ and the offdiagonal elements describe the correlation between each pair of measurements. When the off-diagonal elements are zero, the BLUE method reduces to the familiar weighted mean.

The method constructs a combined result subject to the following conditions:

1. It is a linear combination of the individual estimates $x_{i}$ with weight $\alpha_{i}$ : $\hat{x}=\sum \alpha_{i} x_{i}$

2. provides an unbiassed estimate of $\hat{x}$, which is true if all individual measurements are unbiased and $\sum \alpha_{i}=1$; and

3. provides the best estimate, in the sense of having the smallest possible variance

$$
\sigma^{2}=\vec{\alpha}^{T} \mathbf{E} \vec{\alpha}
$$

where $\vec{\alpha}$ is a vector of the weights $\alpha_{i}$.

The BLUE is found by finding the $n$ values of $\alpha_{i}$ which minimise $\sigma^{2}$. This can be achieved by using the method of Lagrangian multipliers:

$$
\vec{\alpha}=\frac{\mathbf{E}^{-1} \vec{u}}{\vec{u}^{T} \mathbf{E}^{-1} \vec{u}}
$$


Table 8.3: The combination of all analysis channels is shown. The six asymmetries after subtracting the backgrounds and correcting for the acceptance (Corr.) are compared to the prediction. The combined values after background subtraction (Bkg. sub.) are shown as well.

\begin{tabular}{cccc}
\hline & Bkg. sub. (\%) & Corr. (\%) & Predicted (\%) \\
\hline$A^{\ell}$ & $2.9 \pm 6.1 \pm 0.9$ & $2.5 \pm 7.1 \pm 1.4$ & $4.7 \pm 0.1$ \\
$A_{\mathrm{FB}}^{\ell^{+}}$ & $4.5 \pm 6.1 \pm 1.1$ & $4.1 \pm 6.8 \pm 1.1$ & $4.4 \pm 0.2$ \\
$A_{\mathrm{FB}}^{\ell^{-}}$ & $-1.2 \pm 6.1 \pm 1.3$ & $-8.4 \pm 7.4 \pm 2.4$ & $-5.0 \pm 0.2$ \\
$A_{\mathrm{FB}}^{\ell}$ & $3.1 \pm 4.3 \pm 0.8$ & $5.8 \pm 5.1 \pm 1.3$ & $4.7 \pm 0.1$ \\
$A^{\ell \ell}$ & $3.3 \pm 6.0 \pm 1.1$ & $5.3 \pm 7.9 \pm 2.9$ & $6.2 \pm 0.2$ \\
$A_{\mathrm{CP}}^{\ell}$ & $1.8 \pm 4.3 \pm 1.0$ & $-1.8 \pm 5.1 \pm 1.6$ & $-0.3 \pm 0.1$ \\
\hline
\end{tabular}

where $\vec{u}$ is a vector whose $n$ components are all unity and $\mathbf{E}^{-1}$ is the inverse of the error matrix. One can also construct the weighted sum of squares

$$
S=\sum_{i} \sum_{j}\left(\hat{x}-x_{i}\right)\left(\hat{x}-x_{j}\right)\left(\mathbf{E}^{-1}\right)_{i j}
$$

which measures the extent to which the measurements $x_{i}$ are consistent with $\hat{x}$. The weighted sum of squares $S$ is expected to be distributed according to a $\chi^{2}$ distribution with $n-1$ degrees of freedom. This allows us to judge how well the correlated measurements agree with each other.

Both the background subtracted and acceptance corrected values of the individual channels are combined using this procedure. The results of the combination are shown in Table 8.3.

\subsection{Systematic uncertainties}

The same sources of systematic uncertainty are considered as in the previous analysis. Unlike previously we do not make use of the nuisance parameter method, but evaluate systematic uncertainties by using samples of simulated events in which each source of systematic uncertainty is varied in turn, and the analysis repeated. The difference between the predicted asymmetry in this sample and the nominal sample is given as the one standard deviation 
Table 8.4: Systematic uncertainties for the six unfolded asymmetries for the combination of all of the analysis channels.

\begin{tabular}{lcccccc}
\hline & $A^{\ell}(\%)$ & $A_{\mathrm{FB}}^{\ell^{+}}(\%)$ & $A_{\mathrm{FB}}^{\ell^{-}}(\%)$ & $A_{\mathrm{FB}}^{\ell}(\%)$ & $A^{\ell \ell}(\%)$ & $A_{\mathrm{CP}}^{\ell}(\%)$ \\
\cline { 2 - 7 } Jets & 1.1 & 0.8 & 1.7 & 1.0 & 1.5 & 1.2 \\
MC statistics & 0.4 & 0.4 & 0.4 & 0.3 & 0.5 & 0.3 \\
Bkg. norm. & 0.3 & 0.3 & 0.6 & 0.3 & 0.7 & 0.3 \\
Acceptance & 0.7 & 0.2 & 1.5 & 0.7 & 2.3 & 0.9 \\
Total & 1.4 & 1.1 & 2.4 & 1.3 & 2.9 & 1.6 \\
\hline
\end{tabular}

size of the systematic uncertainty. As a result we quote the systematic uncertainties as an uncertainty on the predicted asymmetries and not on the one measured in data.

A more detailed discussion of each systematic uncertainty is given in Section 7.6. The following systematics are considered in this analysis:

- Jet energy scale,

- heavy quark jet energy scale,

- jet identification.

- jet energy resolution,

- vertex confirmation, and

- PDF uncertainties.

In addition there is a systematic uncertainty related to potential mismodelling of the lepton acceptance which is used when correcting the result for acceptance effects. The details of the acceptance correction and how the systematic uncertainty was derived are given in Section 8.4. Systematic uncertainties which effect the normalisation of samples are small and we consider only the largest one which is the uncertainty on the normalisation of the $\mathrm{Z}+$ jets background. Table 8.4 gives a summary of the systematic uncertainties for all the combined value of each of the six asymmetries. 


\subsection{Results}

Six angular asymmetries based on the rapidity distributions of the charged leptons were measured. These asymmetries are related to the angular asymmetries of top quark and antiquark. Reconstruction of the momenta of the top quarks is complicated by the presence of two neutrinos in the dilepton final state, therefore in this analysis the choice was made to use the charged leptons into which the top quarks decay as proxies. The asymmetries are based purely on measurements of the direction of the leptons which is measured with excellent accuracy in the D0 detector. Making a complicated procedure to correct for the resolution of the measured quantities unnecessary.

Combining the three different final states and two run periods, we find the asymmetry $A^{\ell \ell}$ based on the rapidity difference of the charged leptons, to be $A^{\ell \ell}=(3.3 \pm 6.0$ (stat) \pm 1.1 (syst) $) \%$ after subtracting the backgrounds. After correcting for acceptance effects the asymmetry increases to

$$
A^{\ell \ell}=(5.3 \pm 7.9(\text { stat }) \pm 2.9(\text { syst })) \%
$$

which is in agreement with the next-to-leading order QCD prediction of $(6.2 \pm 0.2) \%$.

The asymmetry based on $Q \cdot \eta$, the charge signed rapidity of each lepton, is found to be $A_{\mathrm{FB}}^{\ell}=(3.1 \pm 4.3$ (stat) \pm 0.8 (syst) $) \%$ for the combination of all final states and run periods. Correcting for acceptance effects the asymmetry is

$$
A_{\mathrm{FB}}^{\ell}=(5.8 \pm 5.1(\text { stat }) \pm 1.3(\text { syst })) \%,
$$

compared to the prediction of $(4.7 \pm 0.1) \%$. This asymmetry is measured in the $\ell+$ jets final state as well [10], which is orthogonal to the dilepton final state. The measurement in the $\ell+$ jets final state yields

$$
A_{\mathrm{FB}}^{\ell}=(15.2 \pm 4.0(\text { stat }+ \text { syst })) \% \text {. }
$$

The main uncertainty for both measurements is the statistical uncertainty, which can be reduced by combining the two. Using the BLUE tech- 
nique, treating all systematic uncertainties as fully correlated, we obtain $(11.8 \pm 3.2($ stat + syst $)) \%$. This represents an improvement of about $20 \%$ in the uncertainty compared to the $\ell+$ jets result alone. The consistency between the two individual measurements is $68 \%$. Comparing the combined value to the prediction a disagreement at the level of 2.2 standard deviations is observed.

Some theoretical models aiming to explain the enhanced asymmetry values also predict a net polarisation of the top quarks. Using the $v \mathrm{WT}$ method described in Chapter 7 the distribution of $\cos \theta$ is investigated for the first time. Good agreement between the SM prediction and the data is observed, with a Kolmogorov-Smirnov test probability of $14 \%$.

The CDF and D0 collaborations have performed measurements of the forward-backward asymmetry in the $\ell+$ jets final state. Both reporting asymmetries larger than predicted in NLO QCD. In particular the asymmetry in

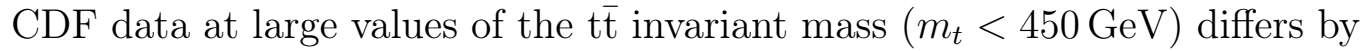
more than three standard deviations from the NLO prediction [6]. While the D0 data show no such excess, the asymmetry $A_{\mathrm{FB}}^{\ell}$ based on the rapidity of the charged lepton deviates from the standard model prediction by about three standard deviations. While the pp initial state puts the ATLAS and CMS collaborations at a disadvantage relative to the Tevatron experiments, their datasets are quickly increasing in size. Both have performed measurements of the difference in angular distributions between top quarks and antiquarks in the $\ell+$ jets final state using asymmetries based on the top quark and antiquark rapidities $[2,37]$ and pseudorapidities [37]. The results are consistent with the standard model expectations. 


\section{Chapter 9}

\section{Conclusion}

With a mass of $m_{t}=173.2 \pm 0.9 \mathrm{GeV}$ the top quark is the heaviest fundamental particle in the standard model. Its short lifetime of $5 \times 10^{-25} \mathrm{~s}$ implies that the top quark decays before a bound state with another quark can be formed. This presents a unique opportunity to study a bare quark, free from hadronisation effects. Here, two analyses of the production and decay mechanism of top quarks are presented.

In the standard model top quarks produced by the strong interaction are unpolarised, however the direction of their spins are correlated. The strength of this correlation depends on the production mechanism, and is precisely predicted in the standard model. Using the direction of the incoming proton beam as reference direction the spins of top pairs produced via gluon fusion are weakly correlated, where as the spins of top pairs produced via quarkantiquark annihilation are strongly correlated. At the Tevatron the dominant production mechanism is quark-antiquark annihilation. Information about the direction of the top quark spin is transmitted to its daughter particles.

Here, their angular distributions are used to measure the strength of the spin correlation. The spin analysing power of the daughter particles varies, with charged leptons having the largest possible analysing power. In this thesis the dilepton final state is chosen as it allows for unambiguous identification of the particle used to measure the correlation strength and a pure sample of events can be selected. The drawback of the dilepton final 
state is that it is under-constrained due to the two neutrinos in the final state. In order to measure the correlation strength it is necessary to reconstruct the four momenta of the top quark and antiquark. This reconstruction is performed using the neutrino weighting algorithm.

Using $5.4 \mathrm{fb}^{-1}$ of data in the dilepton decay channels the strength of the spin correlation is measured using the joint differential angular distribution of the charged lepton decay angles. The correlation strength $C$ is extracted from the data using a maximum likelihood fit which incorporates systematic uncertainties as nuisance parameters. Using the Feldman-Cousins prescription for setting limits close to a physical boundary the spin correlation strength is measured to be:

$$
C=0.10 \pm 0.45
$$

in agreement with the standard model prediction of $C=0.78$. The results' uncertainty is dominated by the size of the data sample used. To reduce the statistical uncertainty a new observable based on a matrix element technique was developed. The new observable reduces the statistical uncertainty by almost $30 \%$. Using both the dilepton and lepton plus jets final state the no spin correlation hypothesis is excluded at more than three standard deviations for the first time.

The forward-backward asymmetry of top quarks provides a further window into top quark pair production. At next-to-leading order a asymmetry of the order of $5 \%$ is expected in the standard model. The collision of protons and anti-protons at the Tevatron defines a reference direction with respect to which this forward-backward asymmetry can be measured. Instead of directly measuring top quark and antiquark rapidity distributions from which to derive the asymmetry, the rapidity distributions of the charged lepton decay products are used. While it is possible to reconstruct the top quark four momenta, it is a complex procedure. The rapidity distributions of the charged leptons are measured with excellent precision, greatly simplifying the analysis.

Here, using $5.4 \mathrm{fb}^{-1}$ of data, the first measurement performed by the D0 collaboration of the asymmetry $A^{\ell \ell}$ based on the rapidity difference of the 
charged leptons, and the asymmetry $A_{\mathrm{FB}}^{\ell}$ based on $Q \cdot \eta$ is presented. After subtracting the backgrounds we measure $A^{\ell \ell}=(3.3 \pm 6.0$ (stat) \pm 1.1 (syst) $) \%$. After correcting for acceptance effects the asymmetry increases to

$$
A^{\ell \ell}=(5.3 \pm 7.9(\text { stat }) \pm 2.9(\text { syst })) \%,
$$

which is in agreement with the NLO QCD prediction of $(6.2 \pm 0.2) \%$.

A further asymmetry based on $Q \cdot \eta$, the charge signed rapidity of each lepton, is measured to be $A_{\mathrm{FB}}^{\ell}=(3.1 \pm 4.3$ (stat) \pm 0.8 (syst) $) \%$. Correcting for acceptance effects the asymmetry rises to

$$
A_{\mathrm{FB}}^{\ell}=(5.8 \pm 5.1 \text { (stat) } \pm 1.3(\text { syst })) \%
$$

compared to the standard model prediction of $(4.7 \pm 0.1) \%$.

Both measurements presented in this thesis are sensitive to the production mechanism of the top quark pair. While about $85 \%$ of top quark pairs are produced via quark antiquark annihilation and gluon fusion contribution $15 \%$ at the Tevatron, the situation is nearly exactly reversed at the LHC. Furthermore the precision of both analyses is limited by the data size used. For these reasons it is important that both are repeated using the full dataset of the D0 experiment, which will reduce the statistical uncertainty by a factor of $\approx \sqrt{2}$. 


\section{Appendix A}

\section{Control plots}

This appendix shows several plots comparing the data to simulation for each of the three final states, split by run period. Variables related to the transverse momentum $p_{T}$ of the charged leptons and jets, as well as their pseudo rapidity $\eta$ are shown. Plots are given both for the stage of the basic and final selection stages. Figure A.1 describes the colour scheme used in all figures.

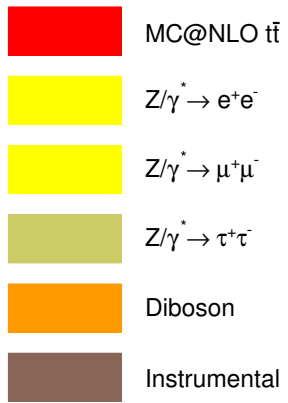

Figure A.1: Legend of the colour scheme used through out this appendix. 


\section{A.1 Run-IIa dielectron channel}
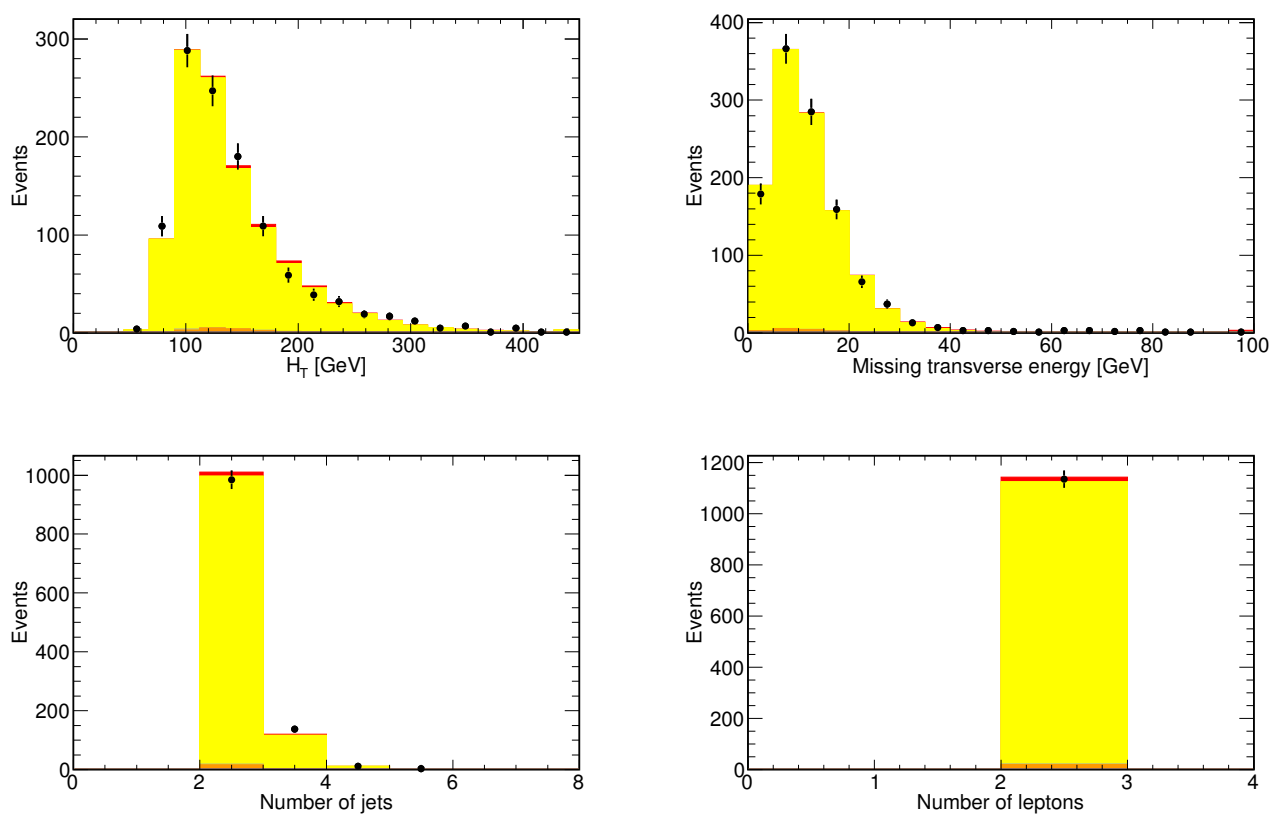

Figure A.2: $H_{T}$, missing transverse energy, number of jets and number of leptons in the Run-IIa ee channel. After requiring two leptons and two jets. 

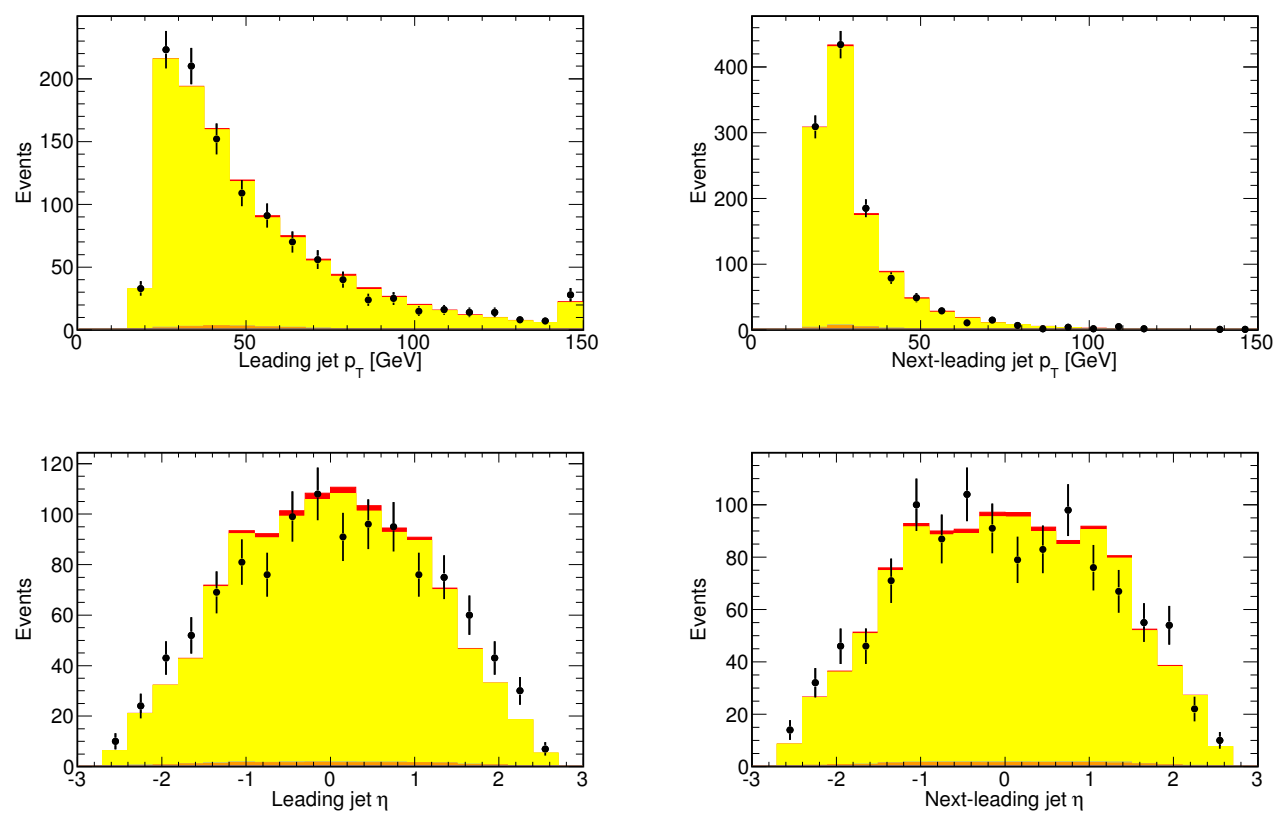

Figure A.3: $\quad p_{T}$ and $\eta$ of the first and second leading jet in the Run-IIa ee channel. After requiring two leptons and two jets.
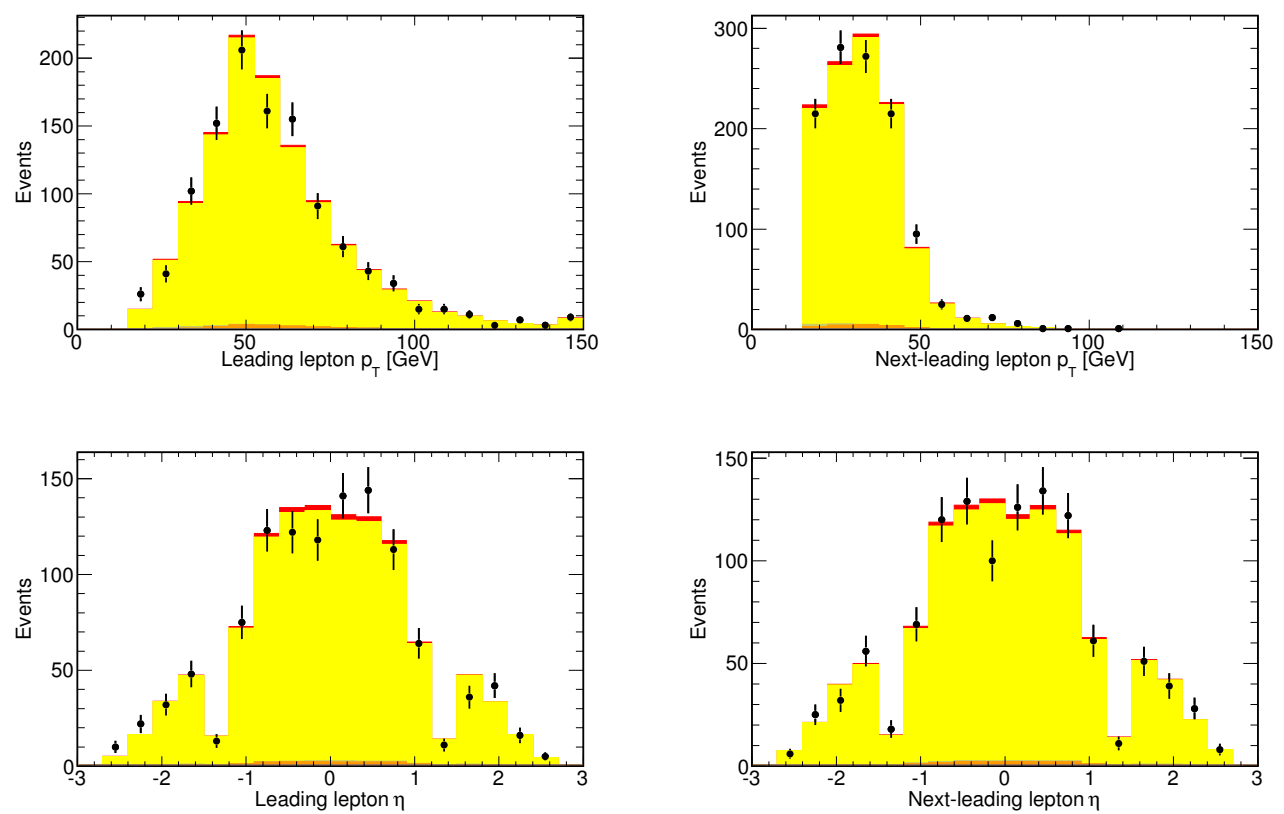

Figure A.4: $p_{T}$ and $\eta$ of the first and second leading lepton in the Run-IIa ee channel. After requiring two leptons and two jets. 

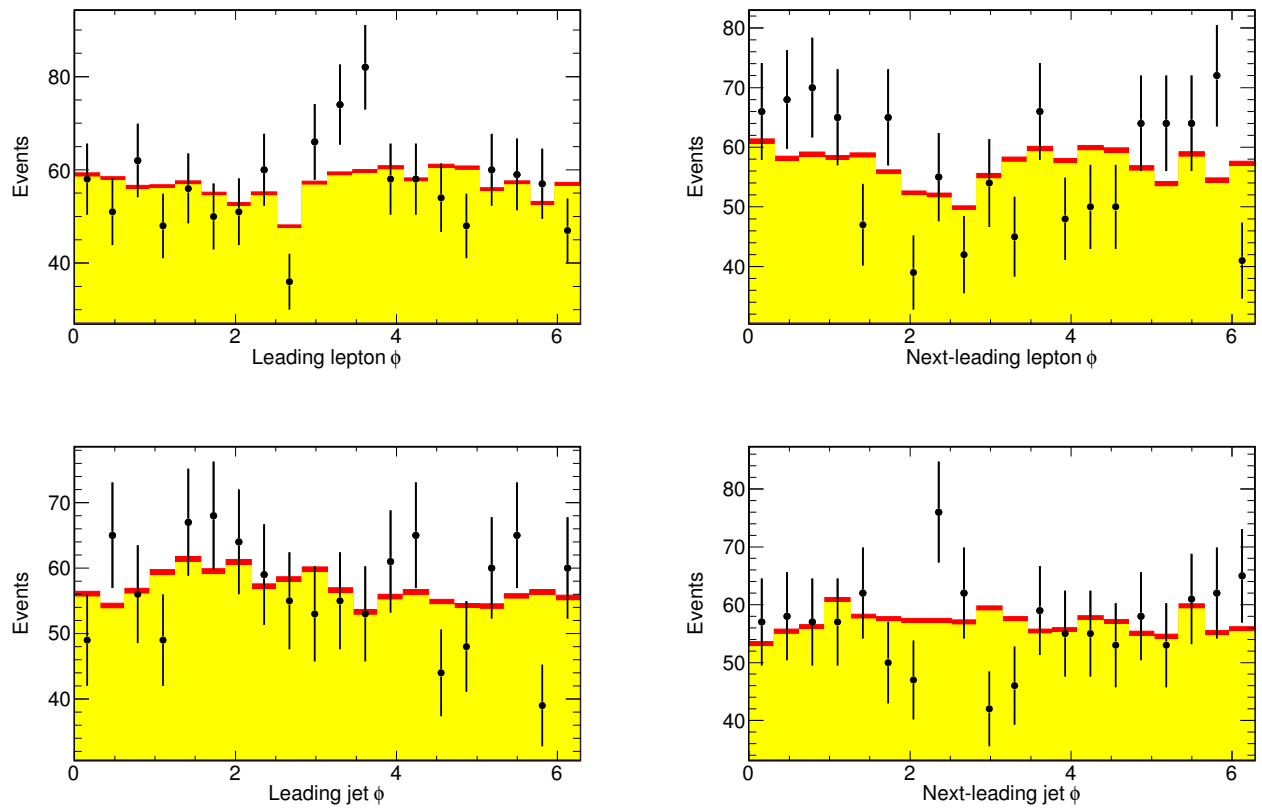

Figure A.5: $\phi$ of the first and second leading lepton and jet in the Run-IIa ee channel. After requiring two leptons and two jets.
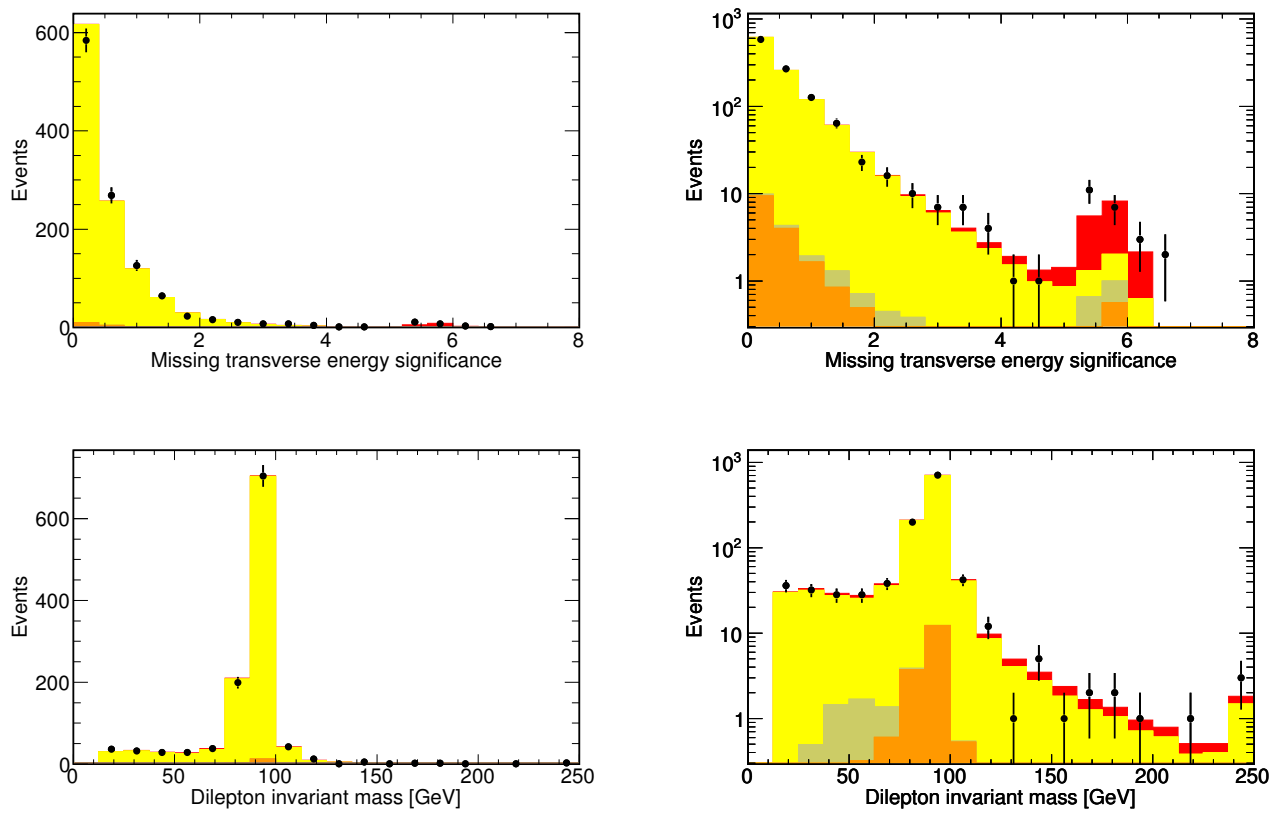

Figure A.6: $\quad \mathbb{E}_{T}$ significance and dilepton invariant mass spectrum in the Run-IIa ee channel. After requiring two leptons and two jets. 

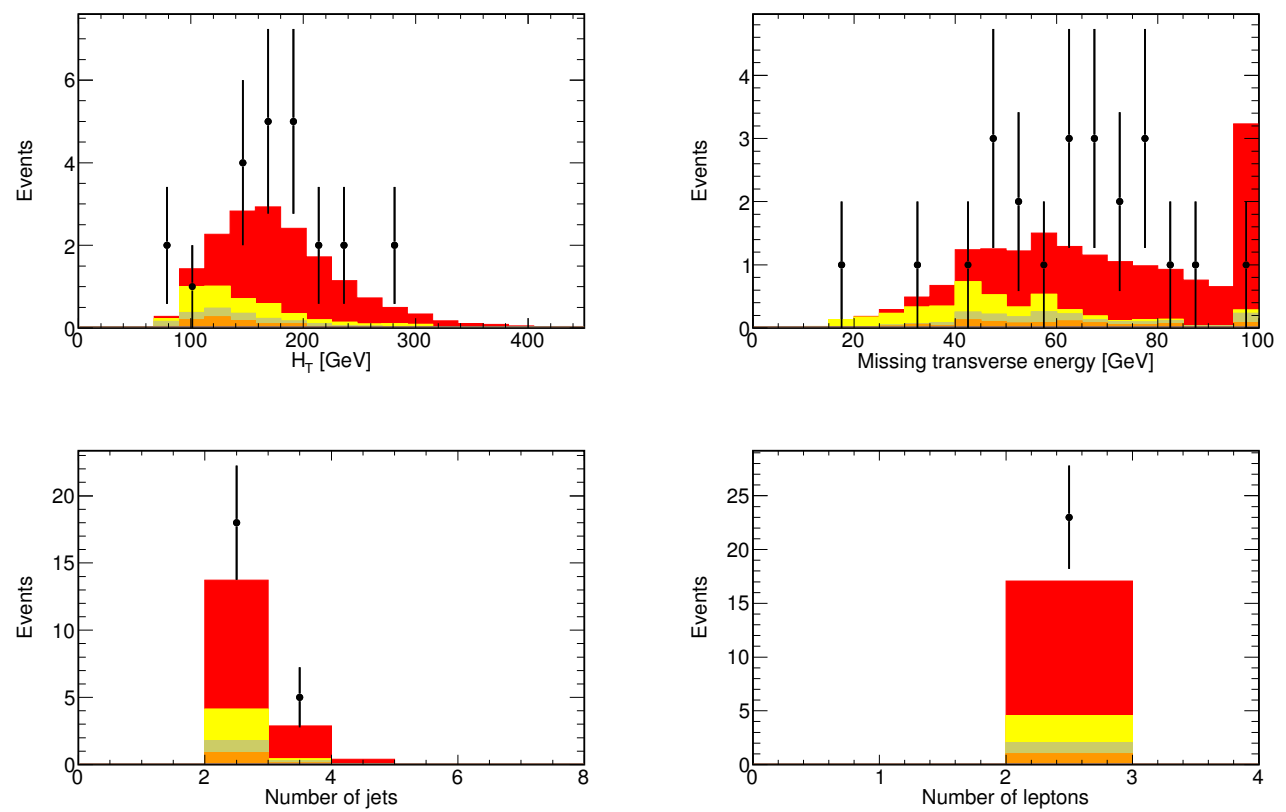

Figure A.7: $H_{T}$, missing transverse energy, number of jets and number of leptons in the Run-IIa ee channel. After the final selection.
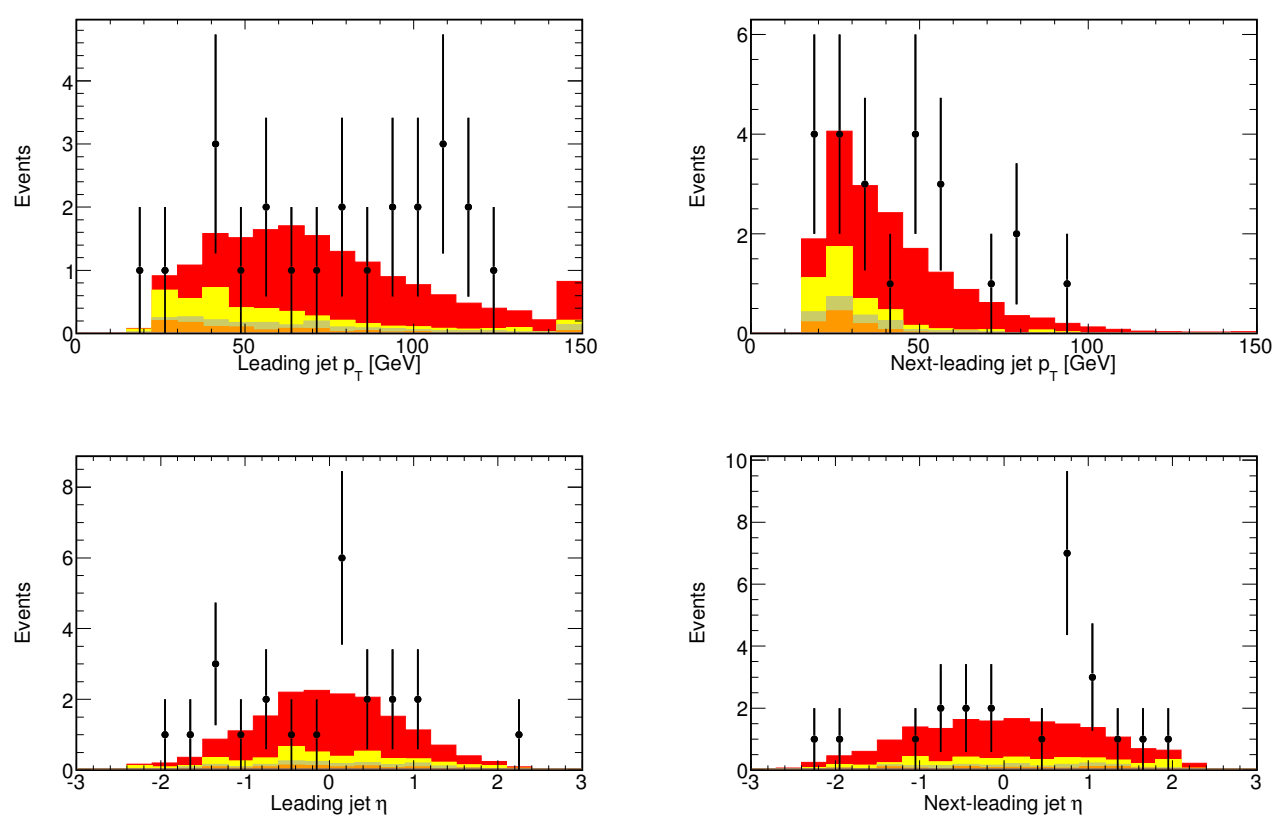

Figure A.8: $\quad p_{T}$ and $\eta$ of the first and second leading jet in the Run-IIa ee channel. After the final selection. 

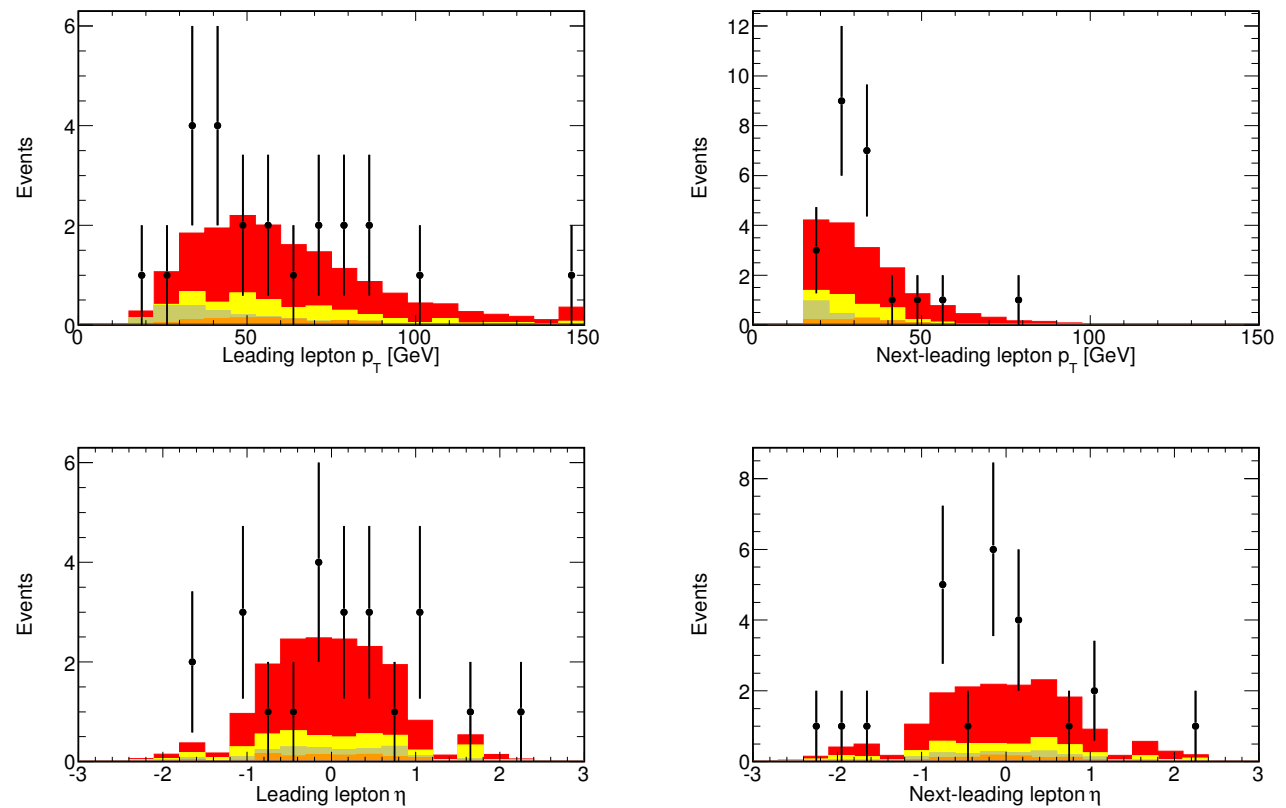

Figure A.9: $p_{T}$ and $\eta$ of the first and second leading lepton in the Run-IIa ee channel. After the final selection.
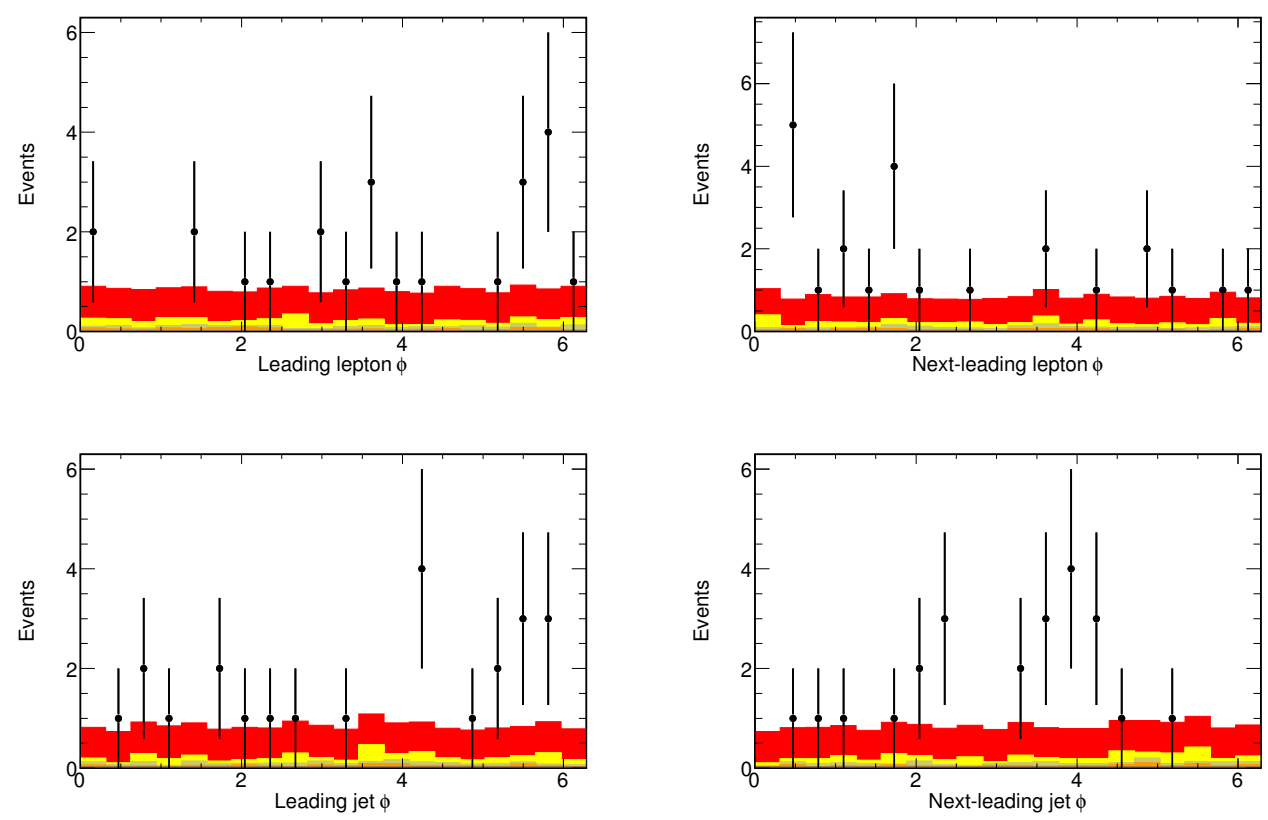

Figure A.10: $\phi$ of the first and second leading lepton and jet in the Run-IIa ee channel. After the final selection. 

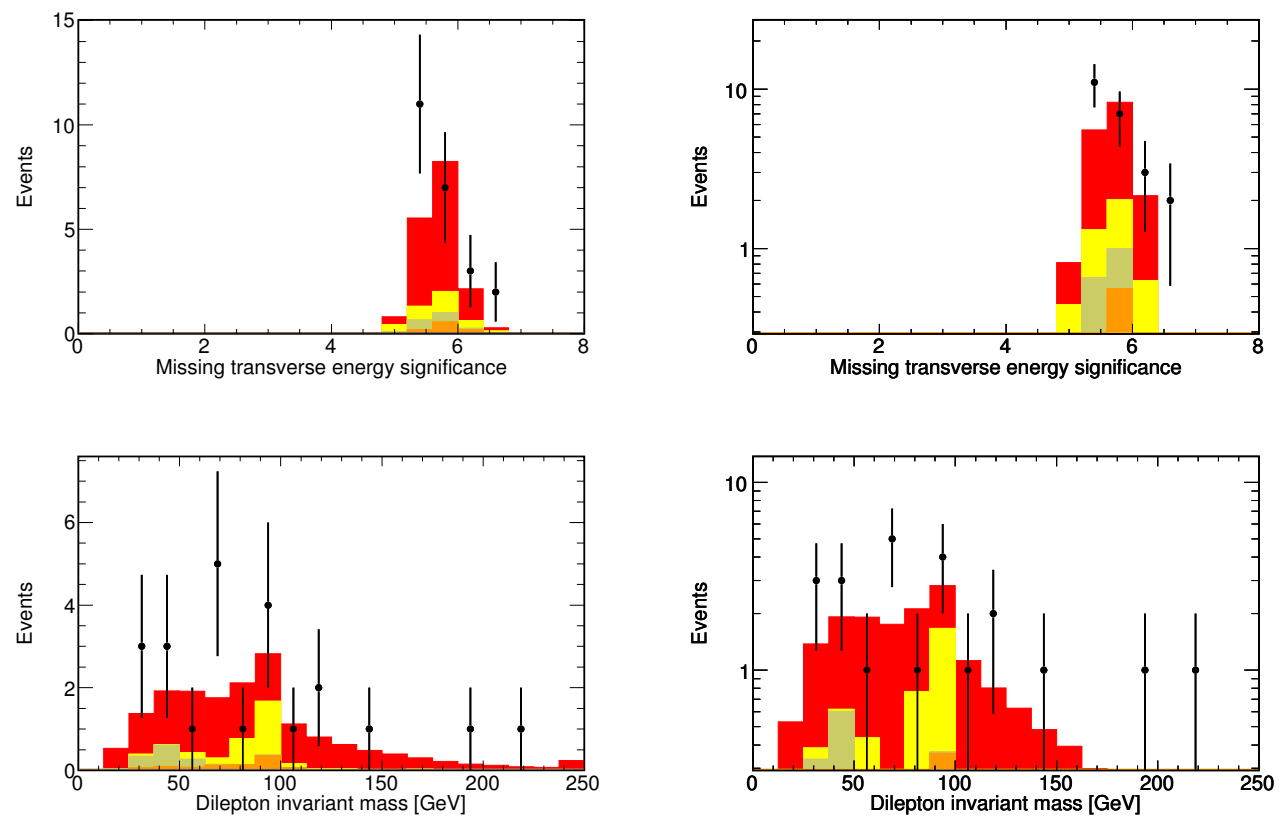

Figure A.11: $\mathbb{E}_{T}$ significance and dilepton invariant mass spectrum in the Run-IIa ee channel. After the final selection. 


\section{A.2 Run-IIb dielectron channel}
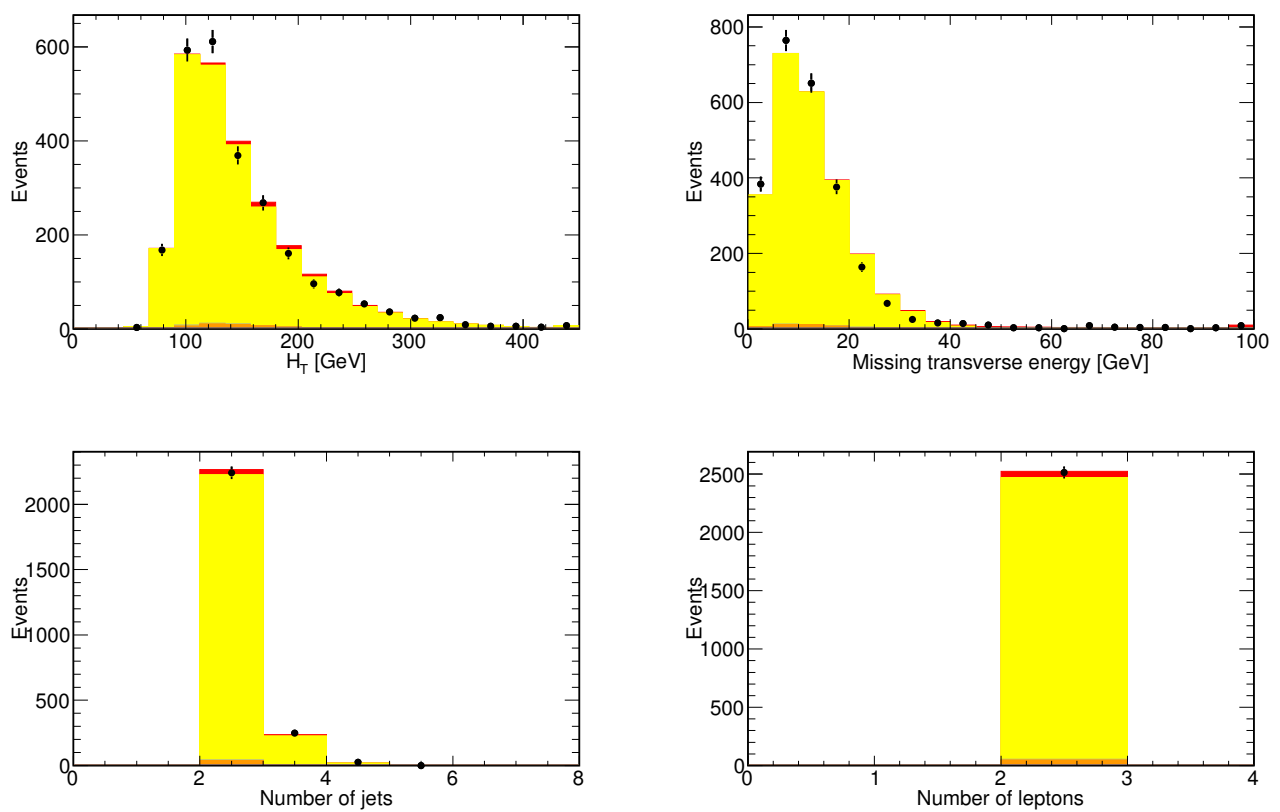

Figure A.12: $H_{T}$, missing transverse energy, number of jets and number of leptons in the Run-IIb ee channel. After requiring two leptons and two jets. 

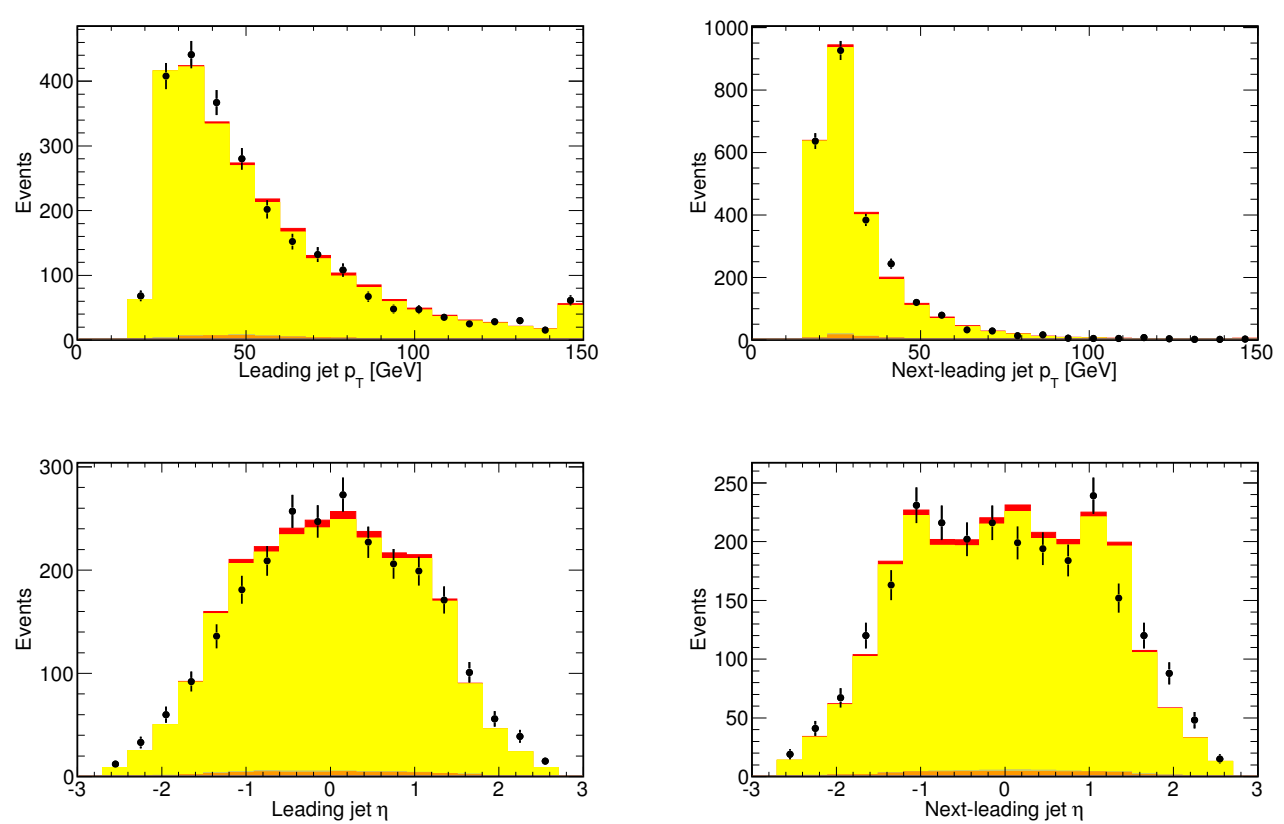

Figure A.13: $p_{T}$ and $\eta$ of the first and second leading jet in the Run-IIb ee channel. After requiring two leptons and two jets.
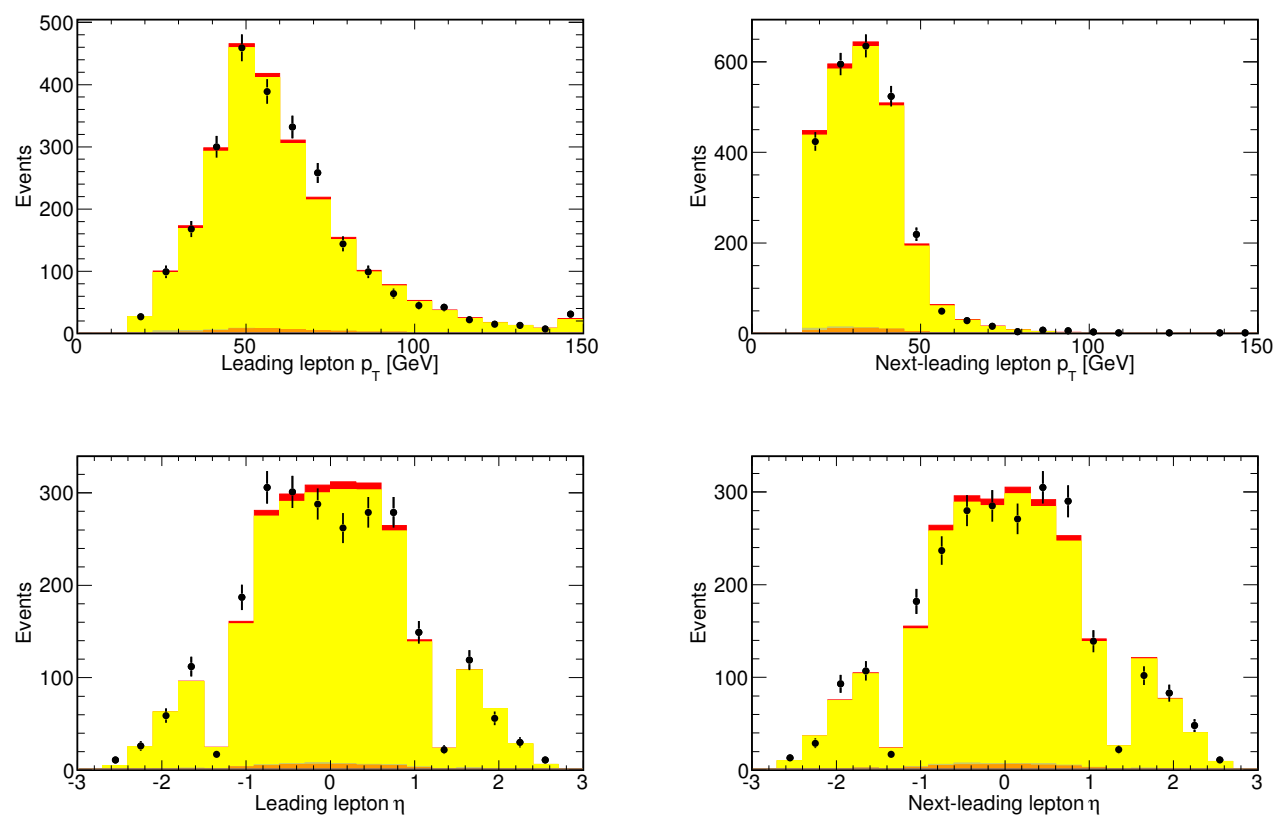

Figure A.14: $p_{T}$ and $\eta$ of the first and second leading lepton in the Run-IIb ee channel. After requiring two leptons and two jets. 

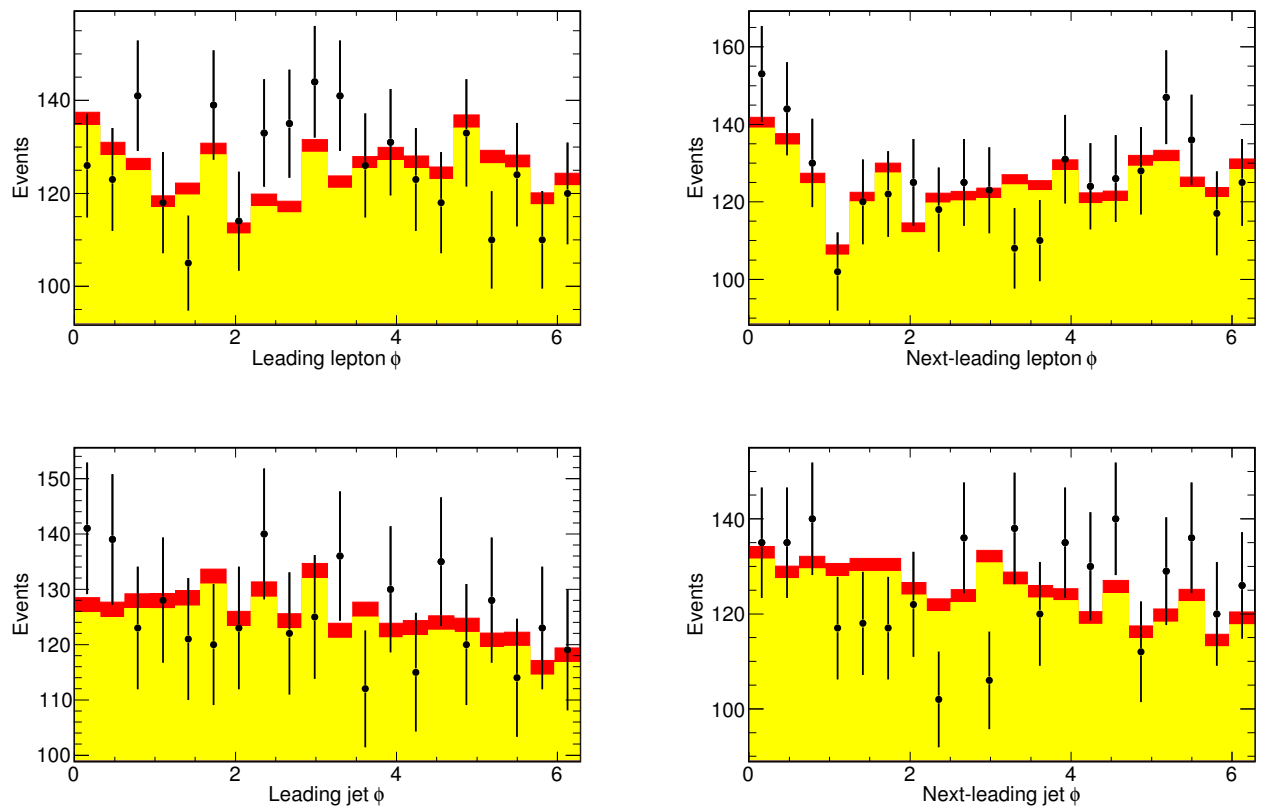

Figure A.15: $\phi$ of the first and second leading lepton and jet in the Run-IIb ee channel. After requiring two leptons and two jets.
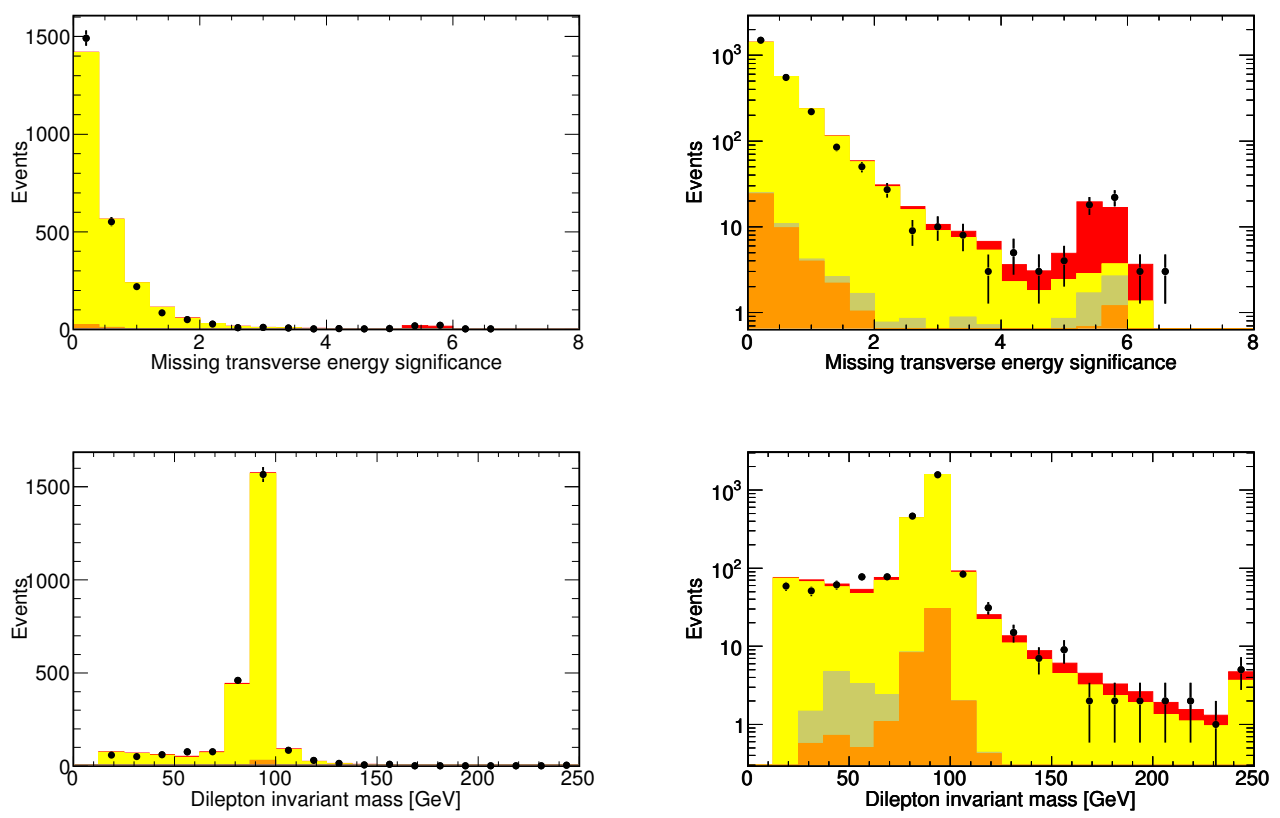

Figure A.16: $\quad \mathbb{E}_{T}$ significance and dilepton invariant mass spectrum in the Run-IIb ee channel. After requiring two leptons and two jets. 

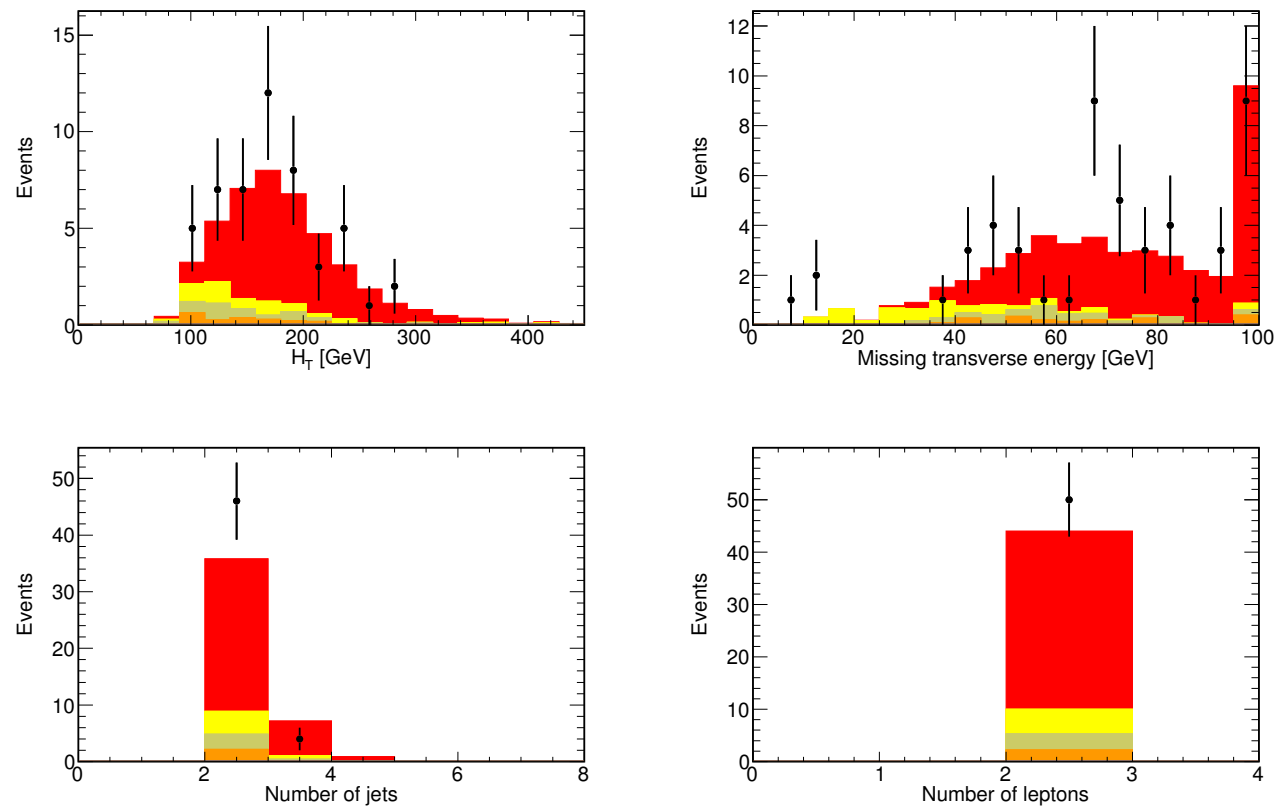

Figure A.17: $H_{T}$, missing transverse energy, number of jets and number of leptons in the Run-IIb ee channel. After the final selection.
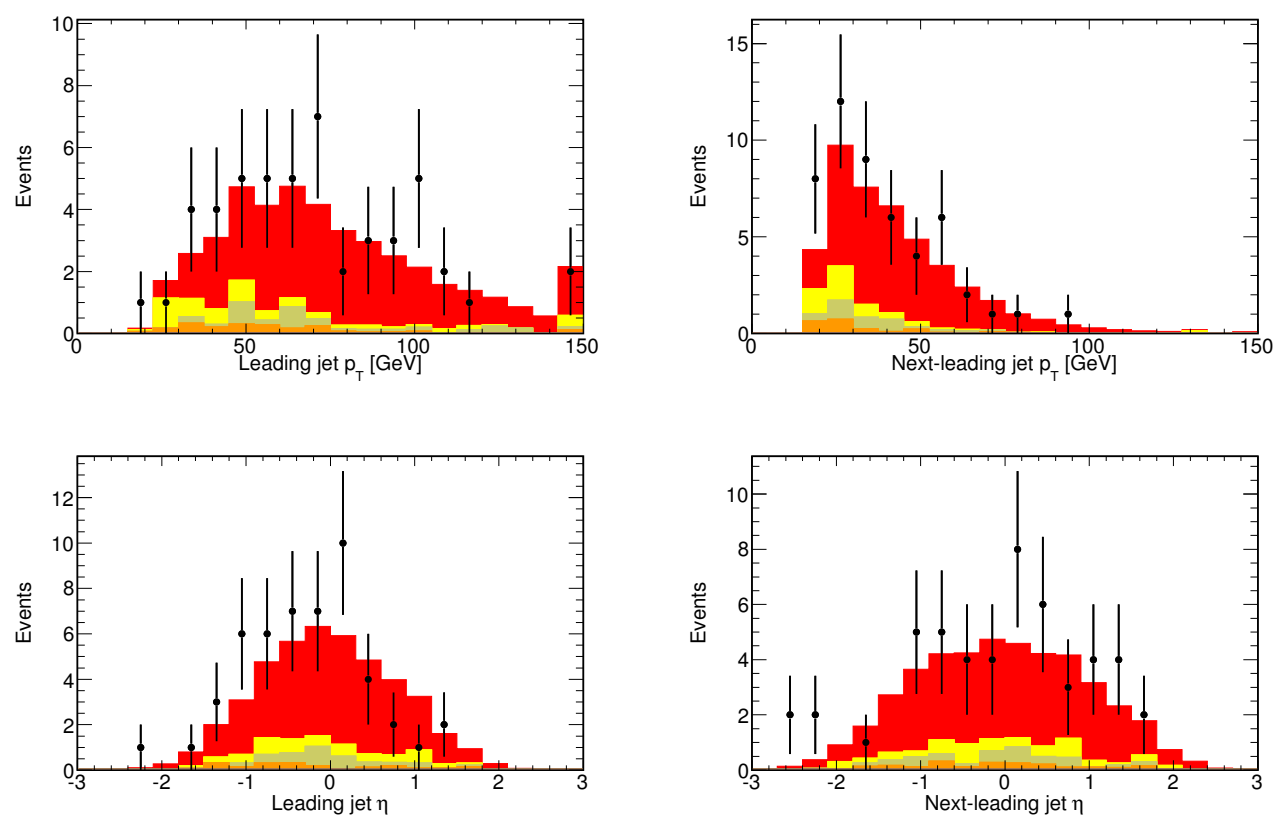

Figure A.18: $p_{T}$ and $\eta$ of the first and second leading jet in the Run-IIb ee channel. After the final selection. 

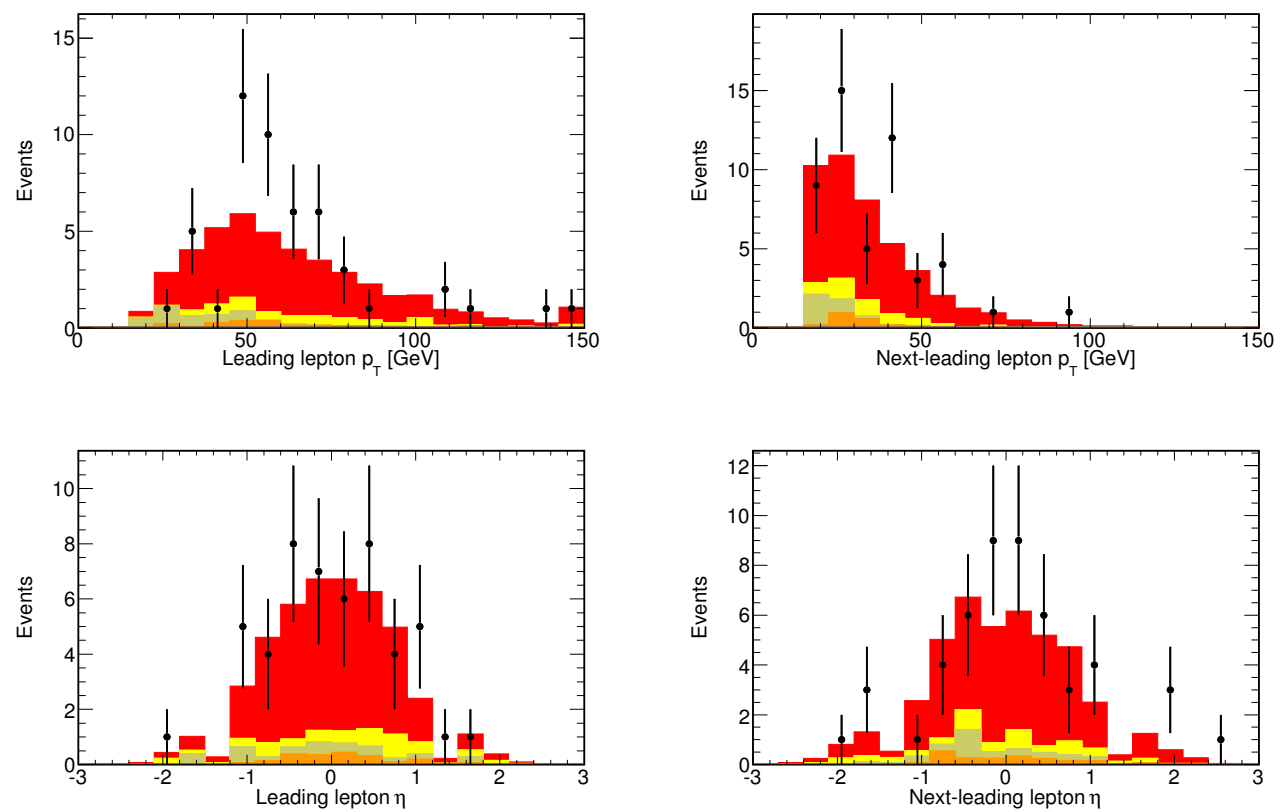

Figure A.19: $p_{T}$ and $\eta$ of the first and second leading lepton in the Run-IIb ee channel. After the final selection.
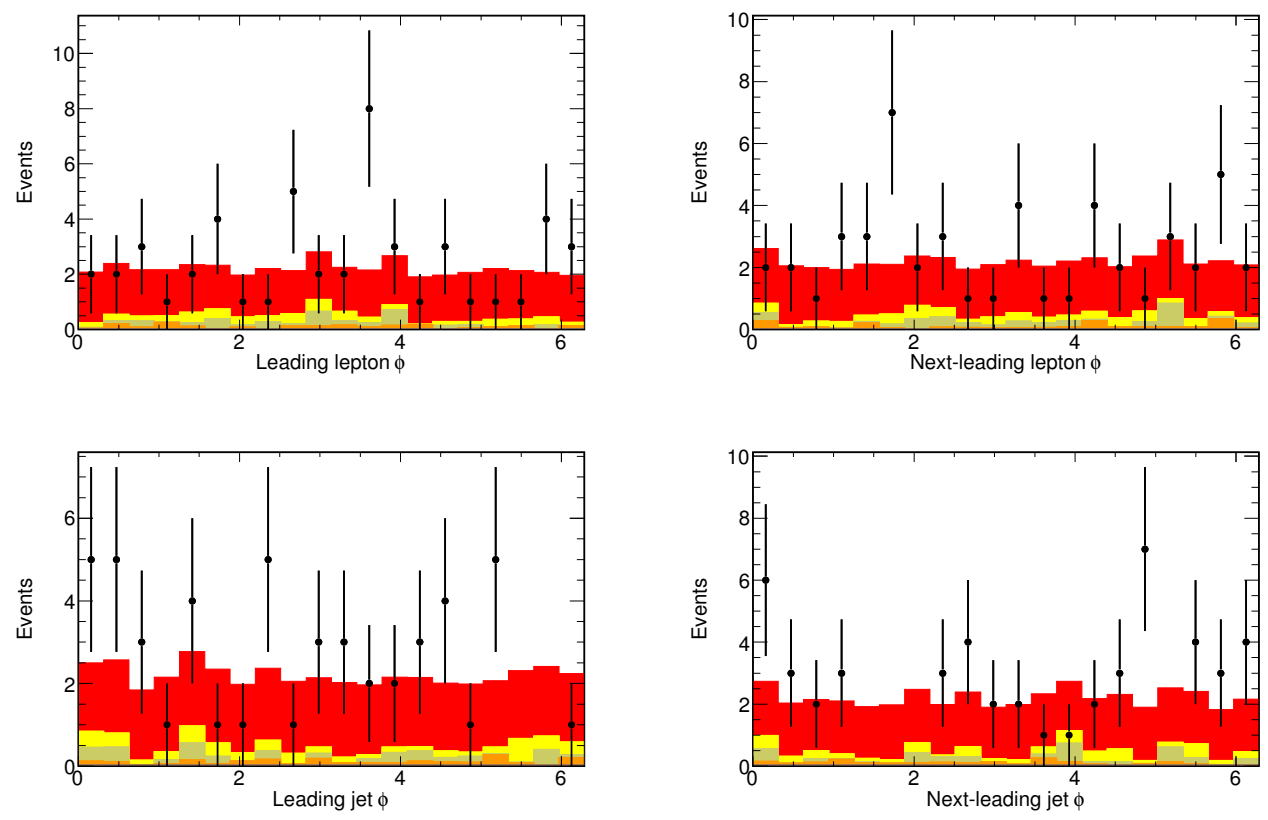

Figure A.20: $\phi$ of the first and second leading lepton and jet in the Run-IIb ee channel. After the final selection. 

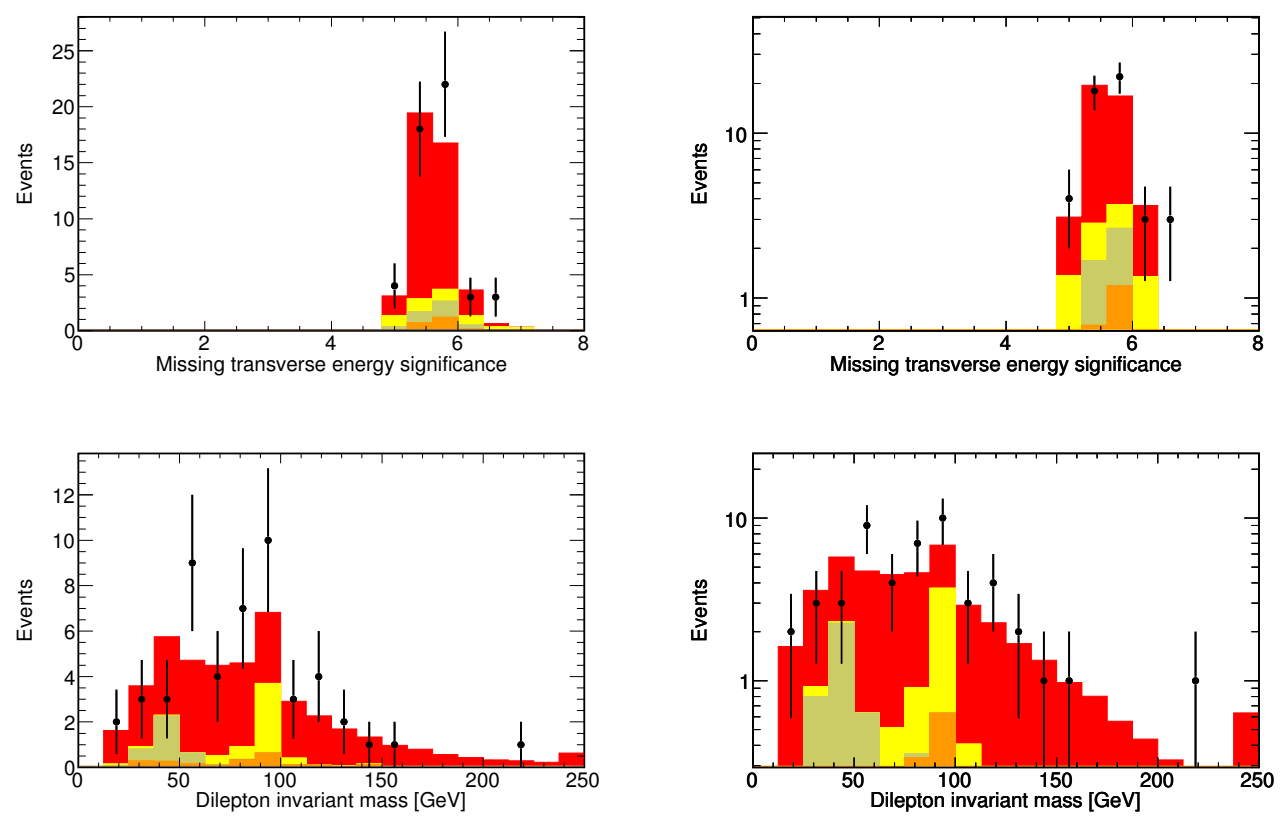

Figure A.21: $\mathbb{E}_{T}$ significance and dilepton invariant mass spectrum in the Run-IIb ee channel. After the final selection. 


\section{A.3 Run-IIa electron-muon channel}
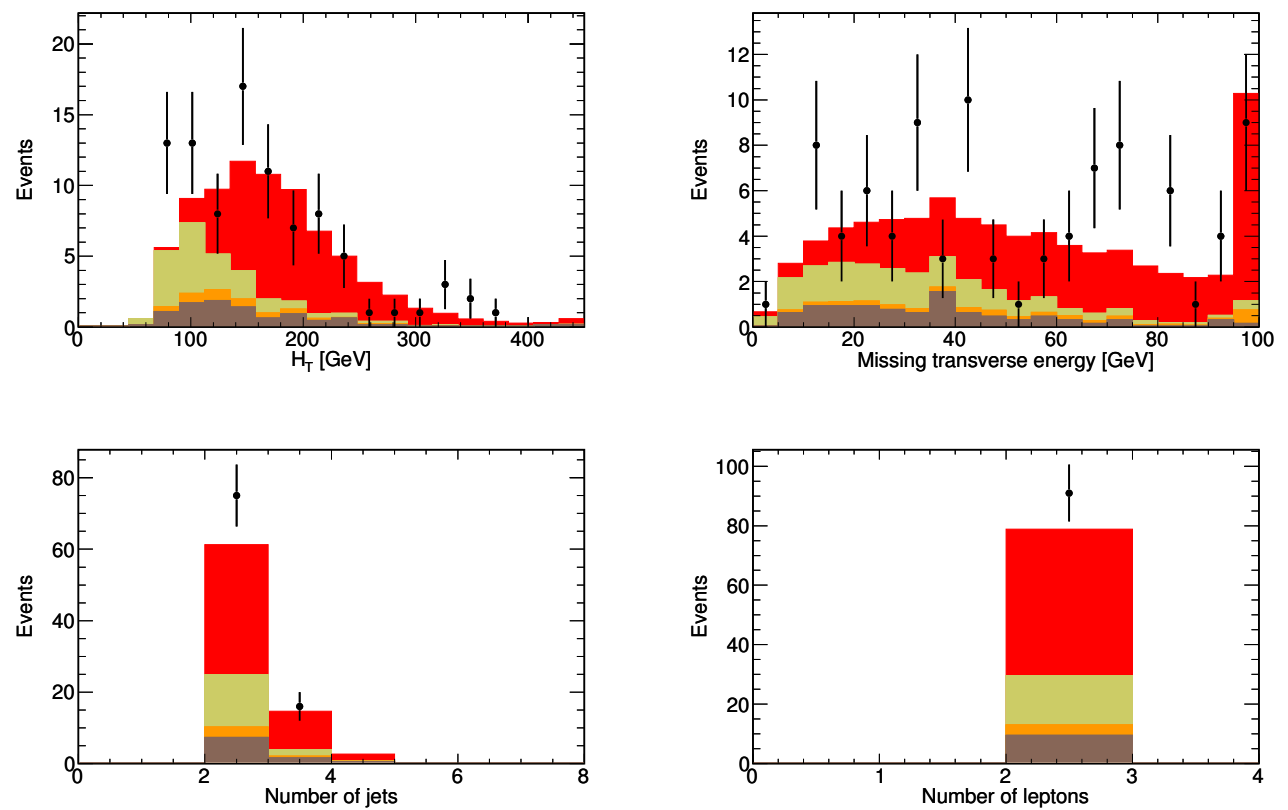

Figure A.22: $H_{T}$, missing transverse energy, number of jets and number of leptons in the Run-IIa e $\mu$ channel. After requiring two leptons and two jets. 

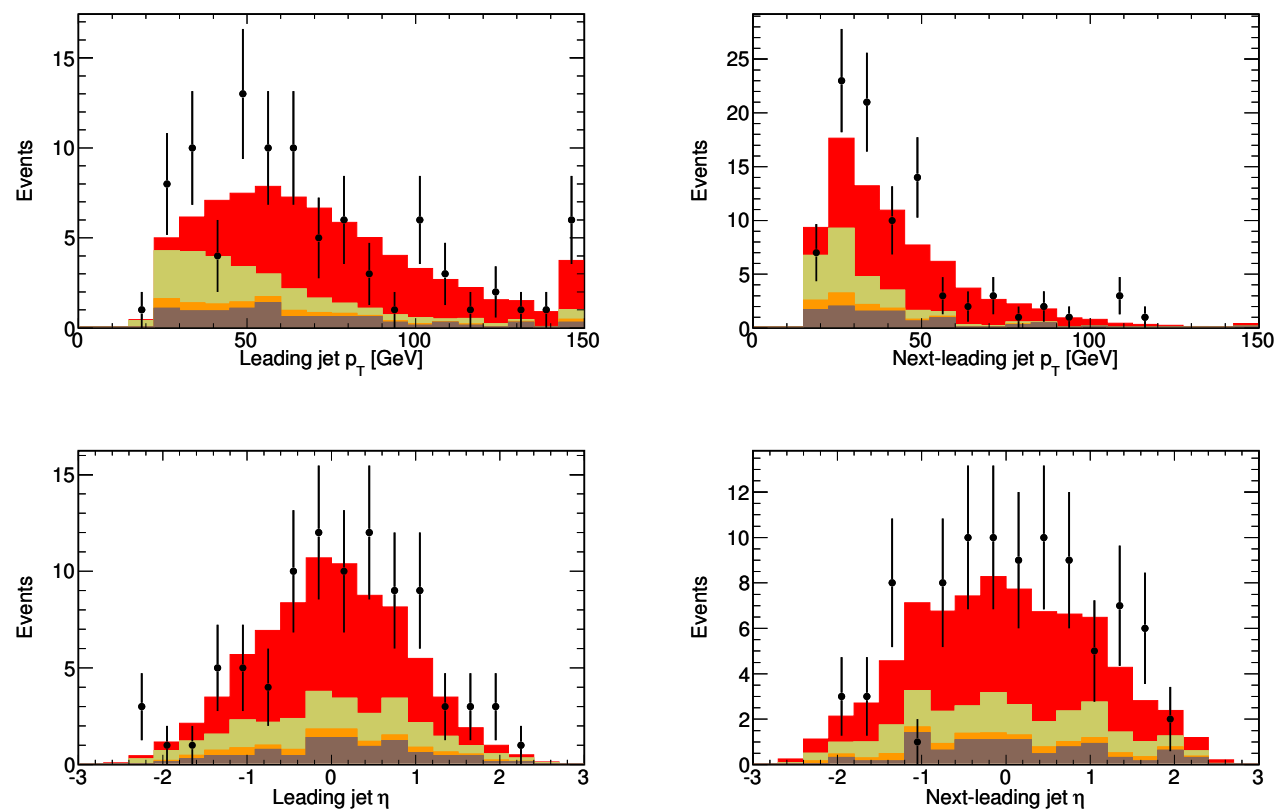

Figure A.23: $p_{T}$ and $\eta$ of the first and second leading jet in the Run-IIa e $\mu$ channel. After requiring two leptons and two jets.
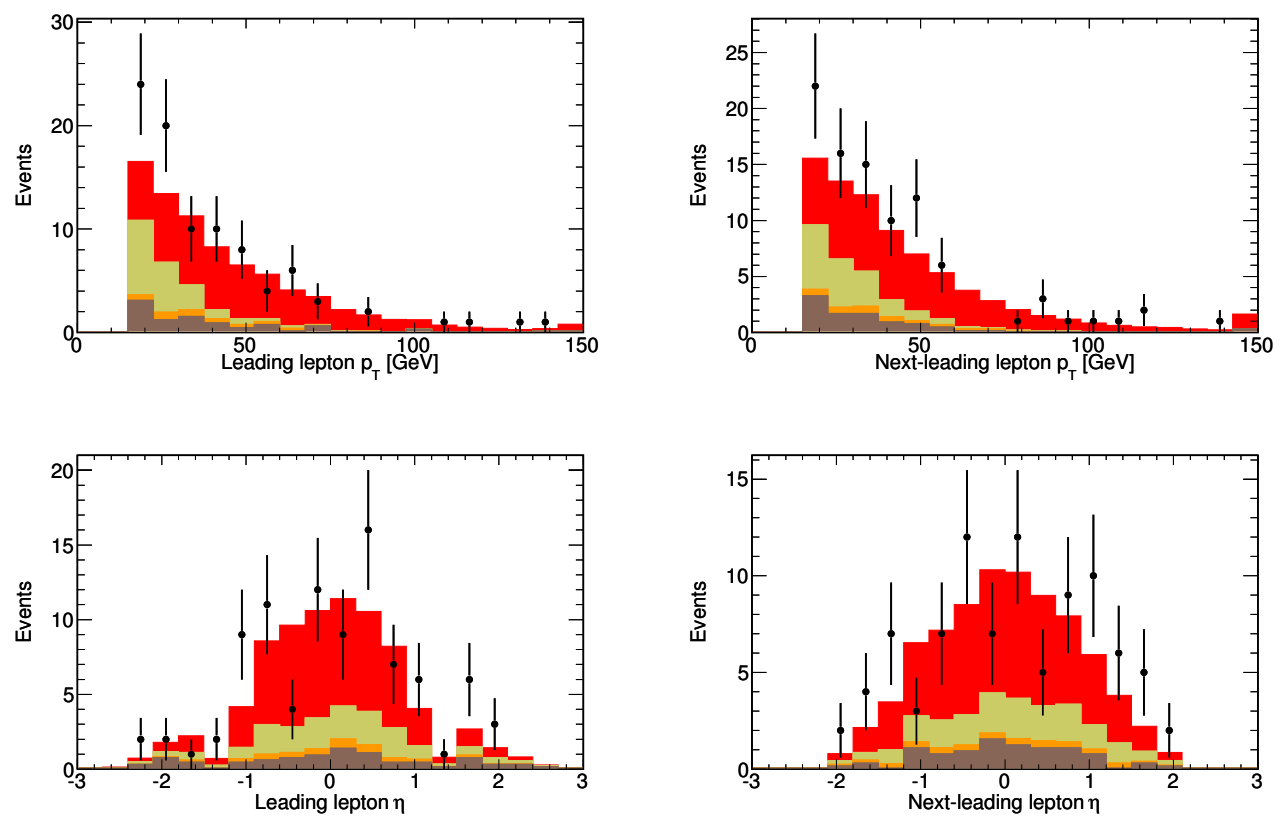

Figure A.24: $p_{T}$ and $\eta$ of the first and second leading lepton in the Run-IIa e $\mu$ channel. After requiring two leptons and two jets. 

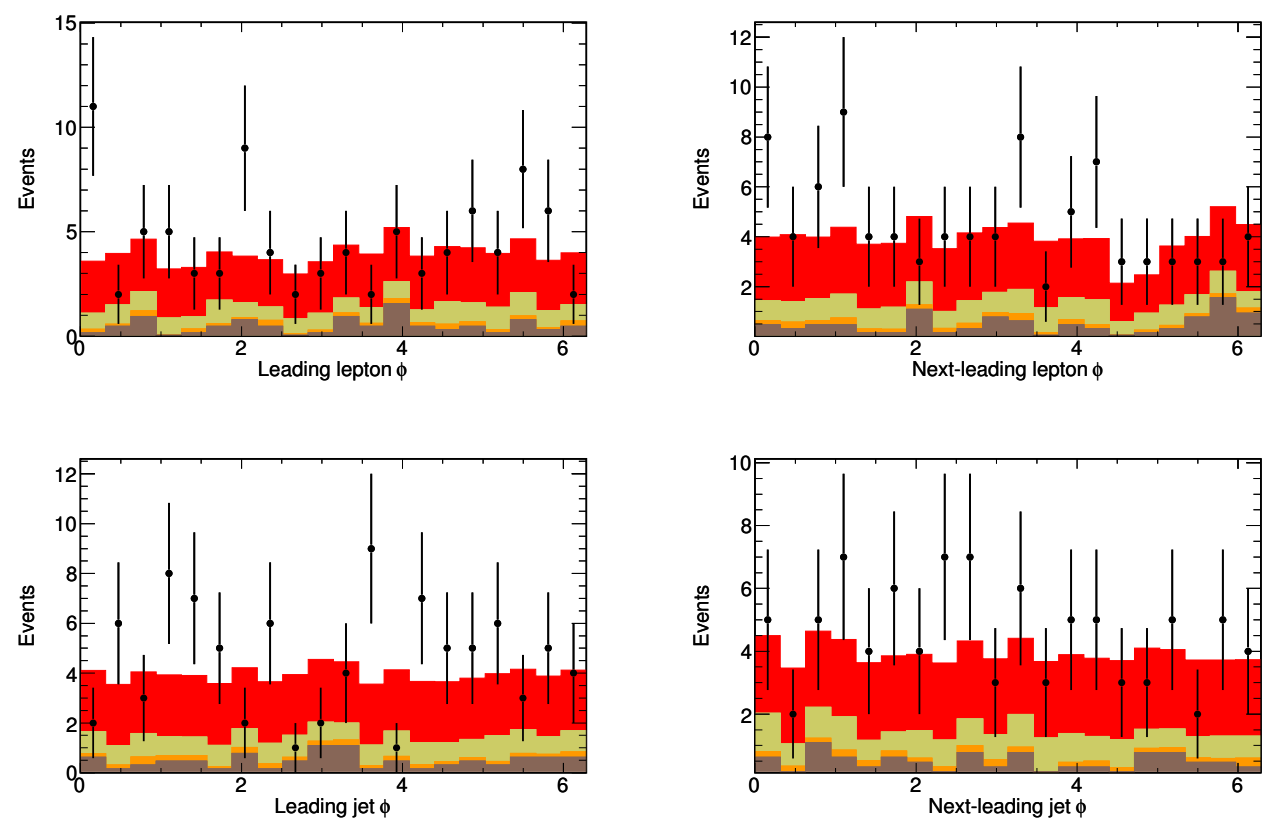

Figure A.25: $\phi$ of the first and second leading lepton and jet in the Run-IIa $\mathrm{e} \mu$ channel. After requiring two leptons and two jets.
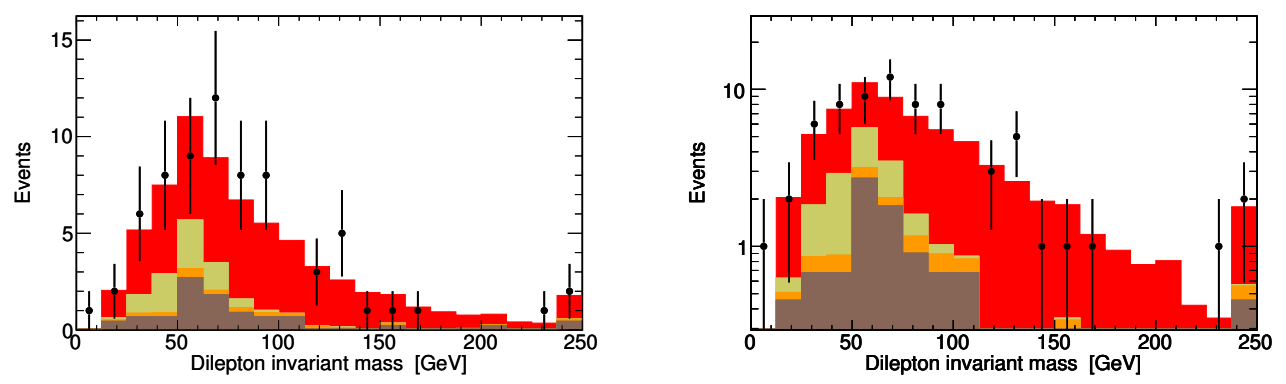

Figure A.26: Dilepton invariant mass spectrum in the Run-IIa e $\mu$ channel. After requiring two leptons and two jets. 

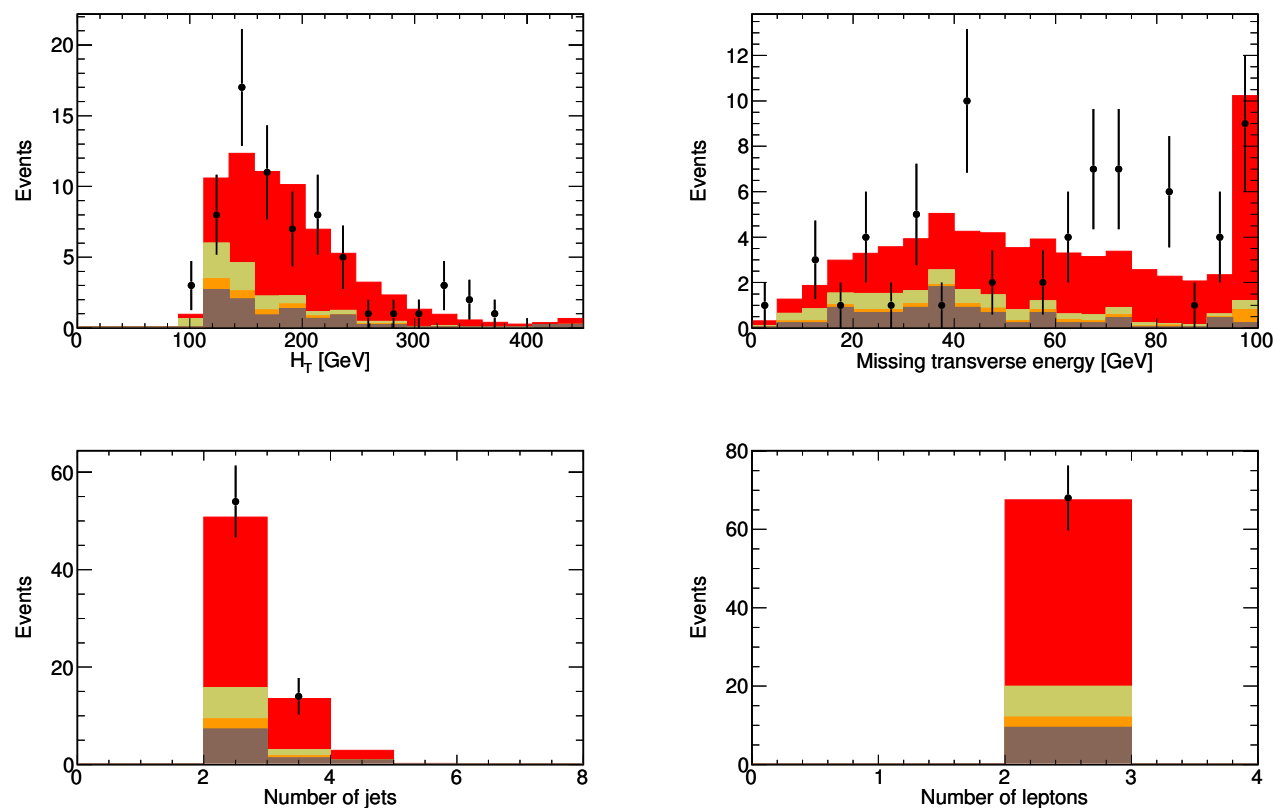

Figure A.27: $H_{T}$, missing transverse energy, number of jets and number of leptons in the Run-IIa e $\mu$ channel. After the final selection.
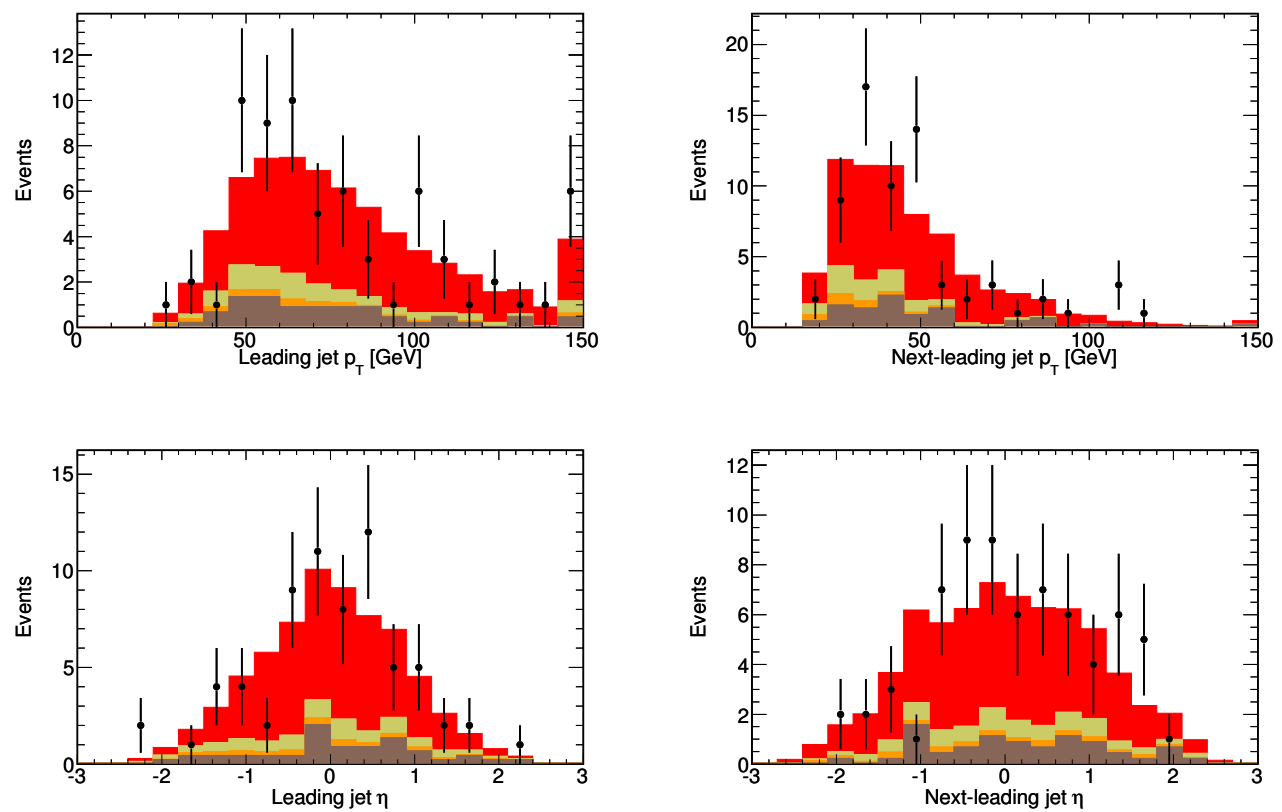

Figure A.28: $p_{T}$ and $\eta$ of the first and second leading jet in the Run-IIa e $\mu$ channel. After the final selection. 

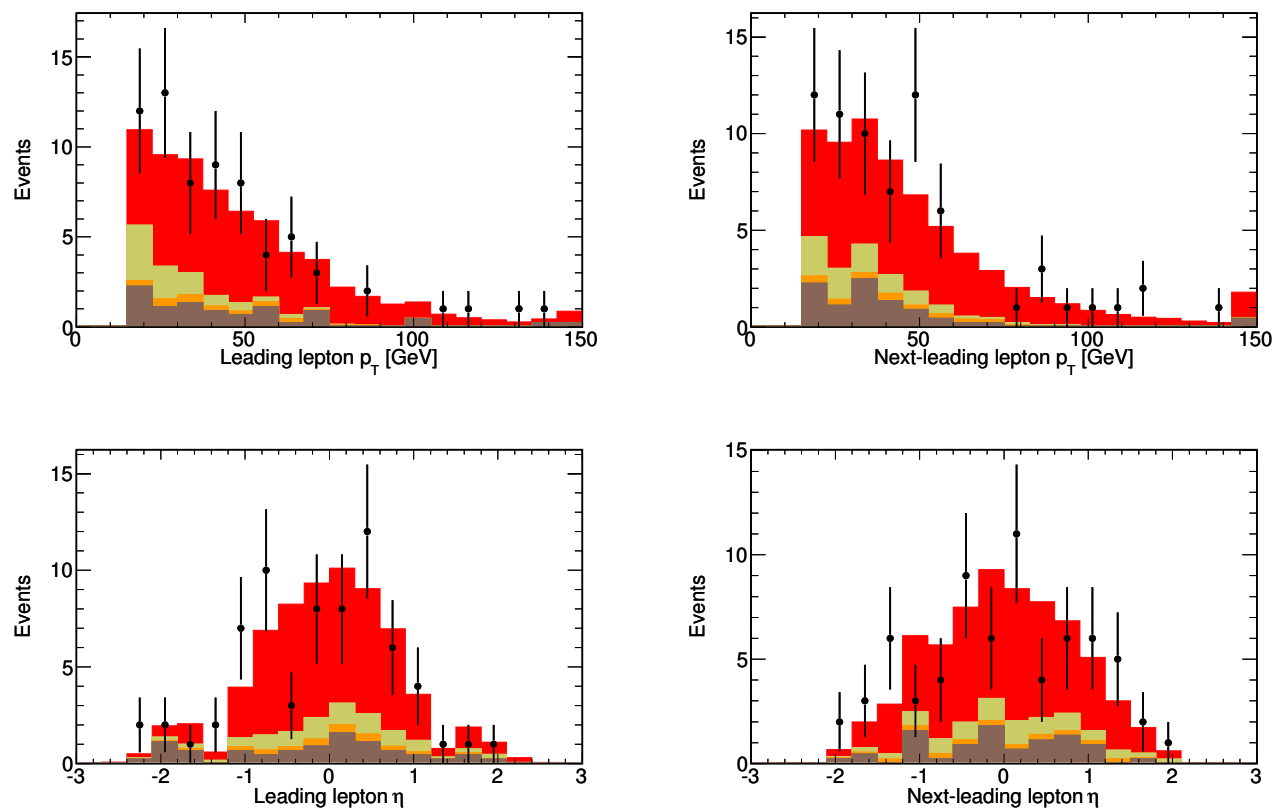

Figure A.29: $p_{T}$ and $\eta$ of the first and second leading lepton in the Run-IIa e $\mu$ channel. After the final selection.
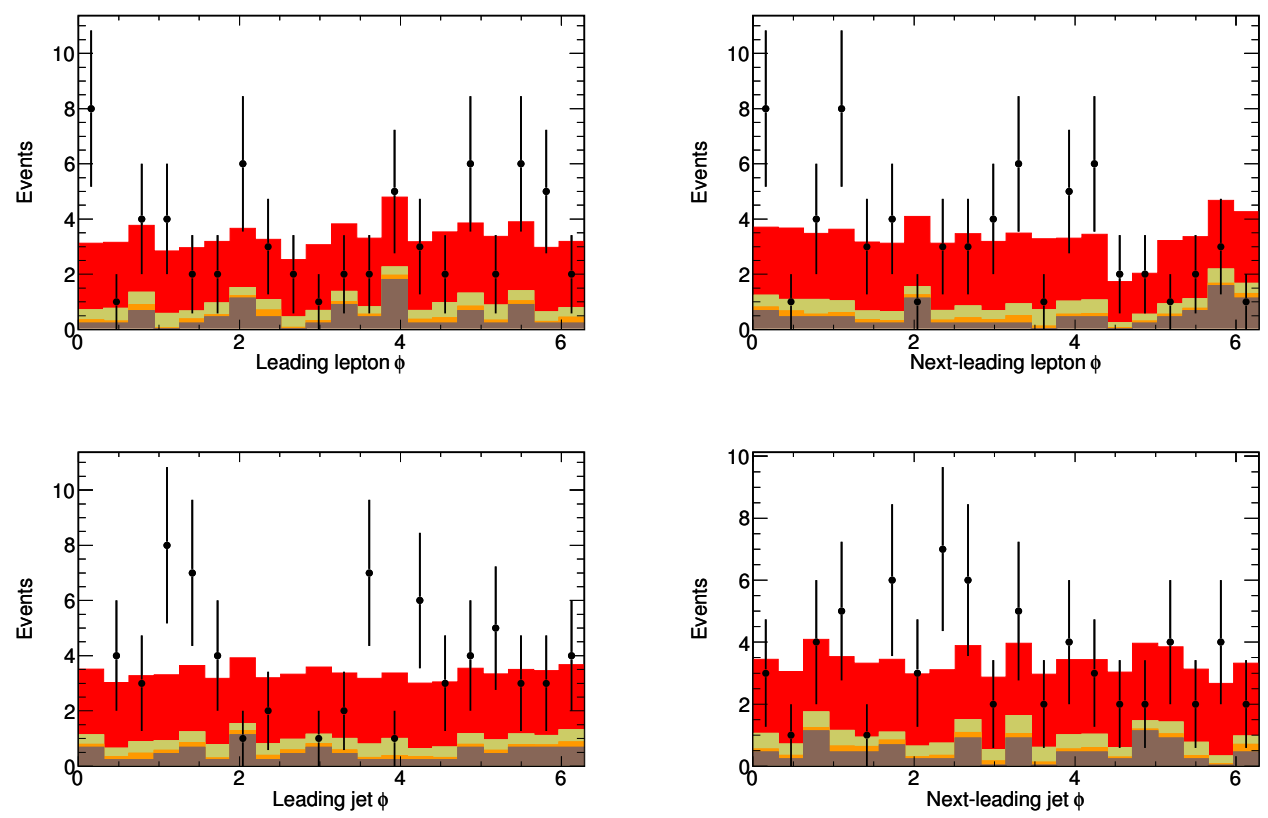

Figure A.30: $\phi$ of the first and second leading lepton and jet in the Run-IIa e $\mu$ channel. After the final selection. 

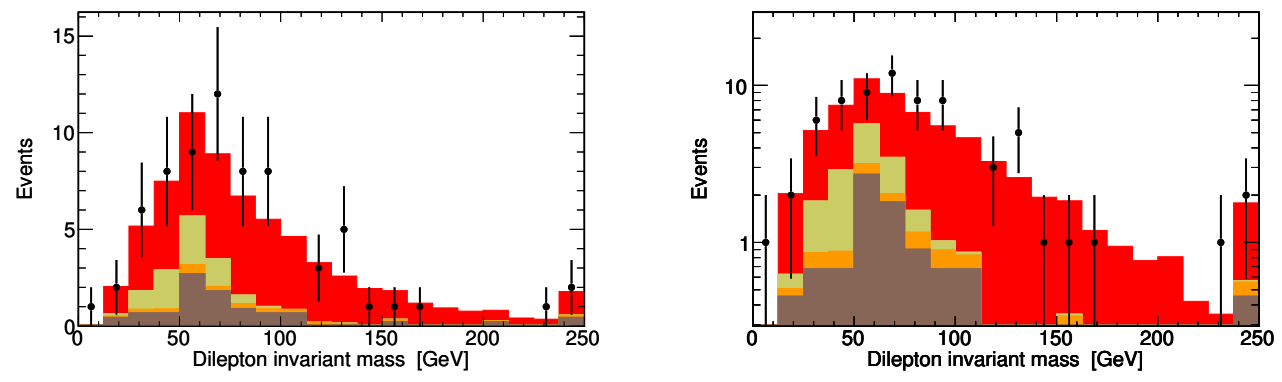

Figure A.31: Dilepton invariant mass spectrum in the Run-IIa e $\mu$ channel. After the final selection. 


\section{A.4 Run-IIb electron-muon channel}
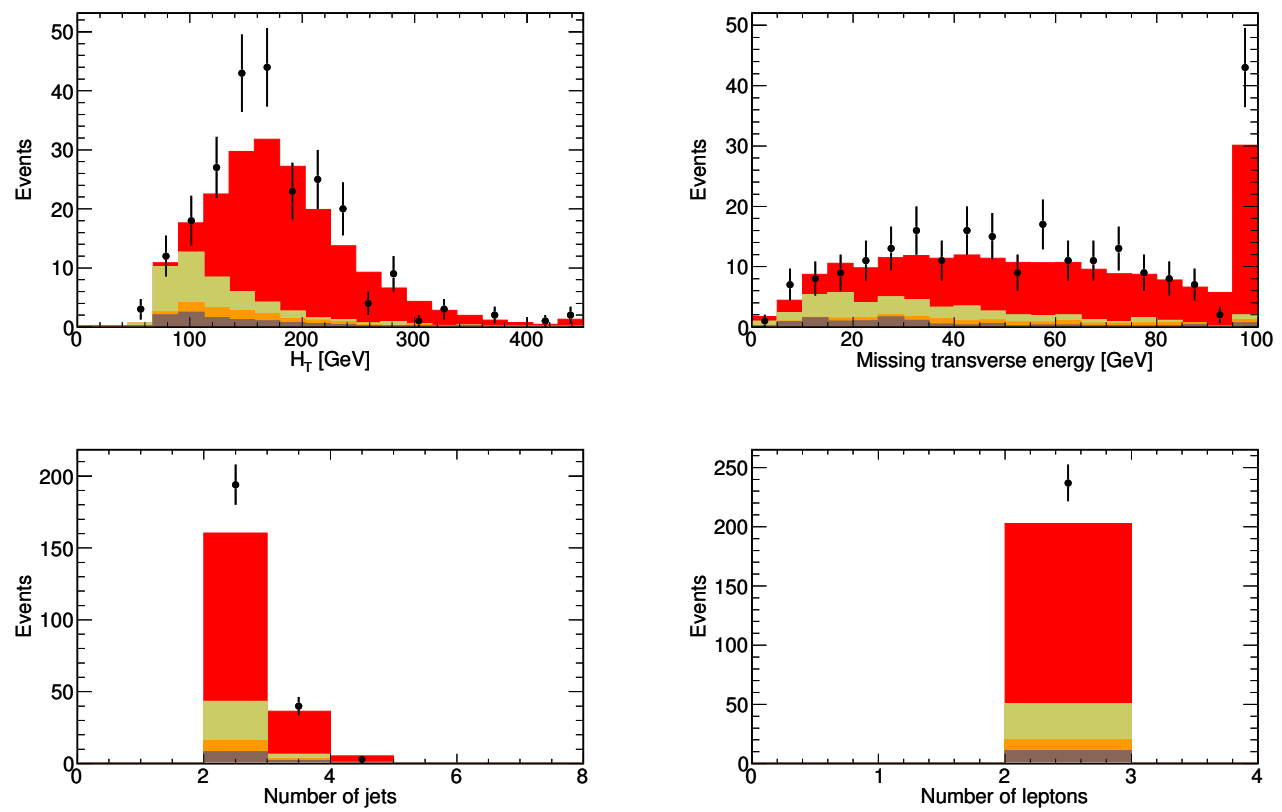

Figure A.32: $H_{T}$, missing transverse energy, number of jets and number of leptons in the Run-IIb e $\mu$ channel. After requiring two leptons and two jets. 

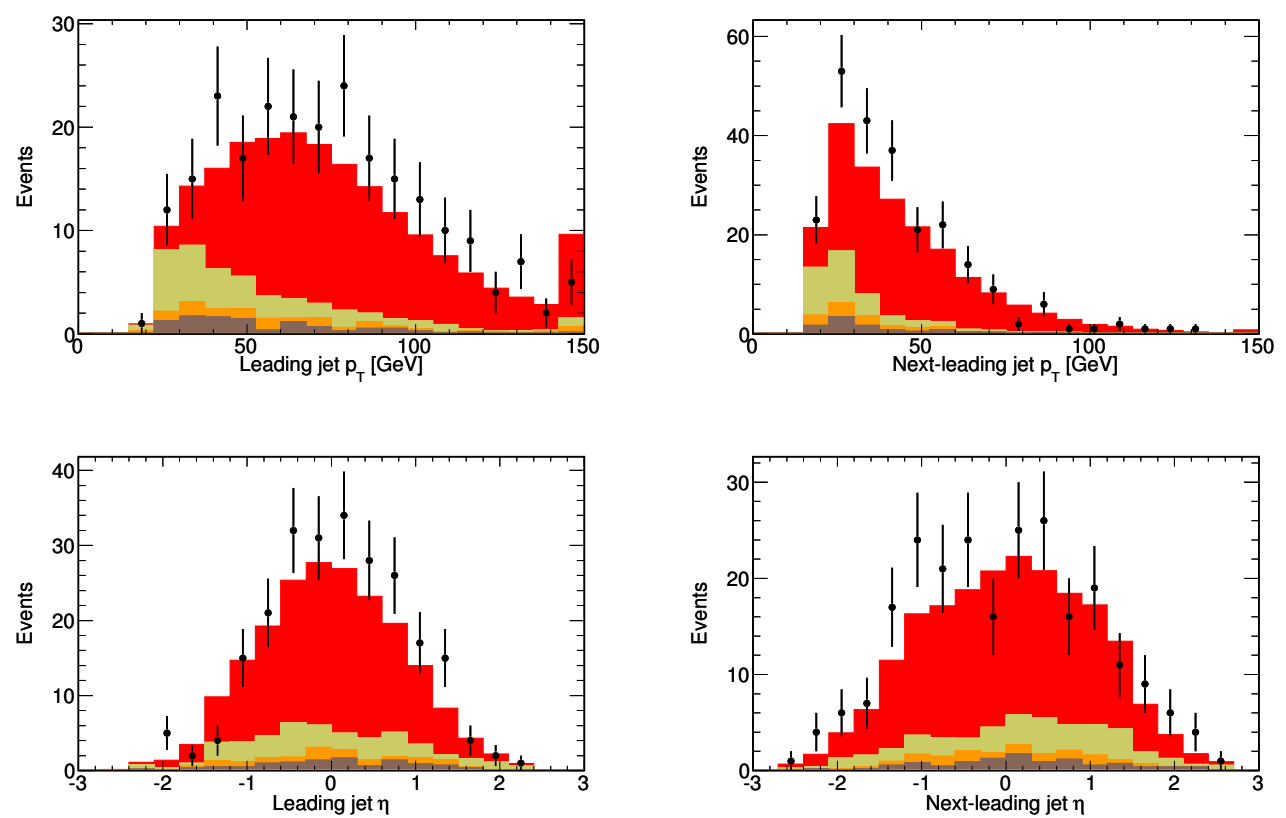

Figure A.33: $p_{T}$ and $\eta$ of the first and second leading jet in the Run-IIb e $\mu$ channel. After requiring two leptons and two jets.
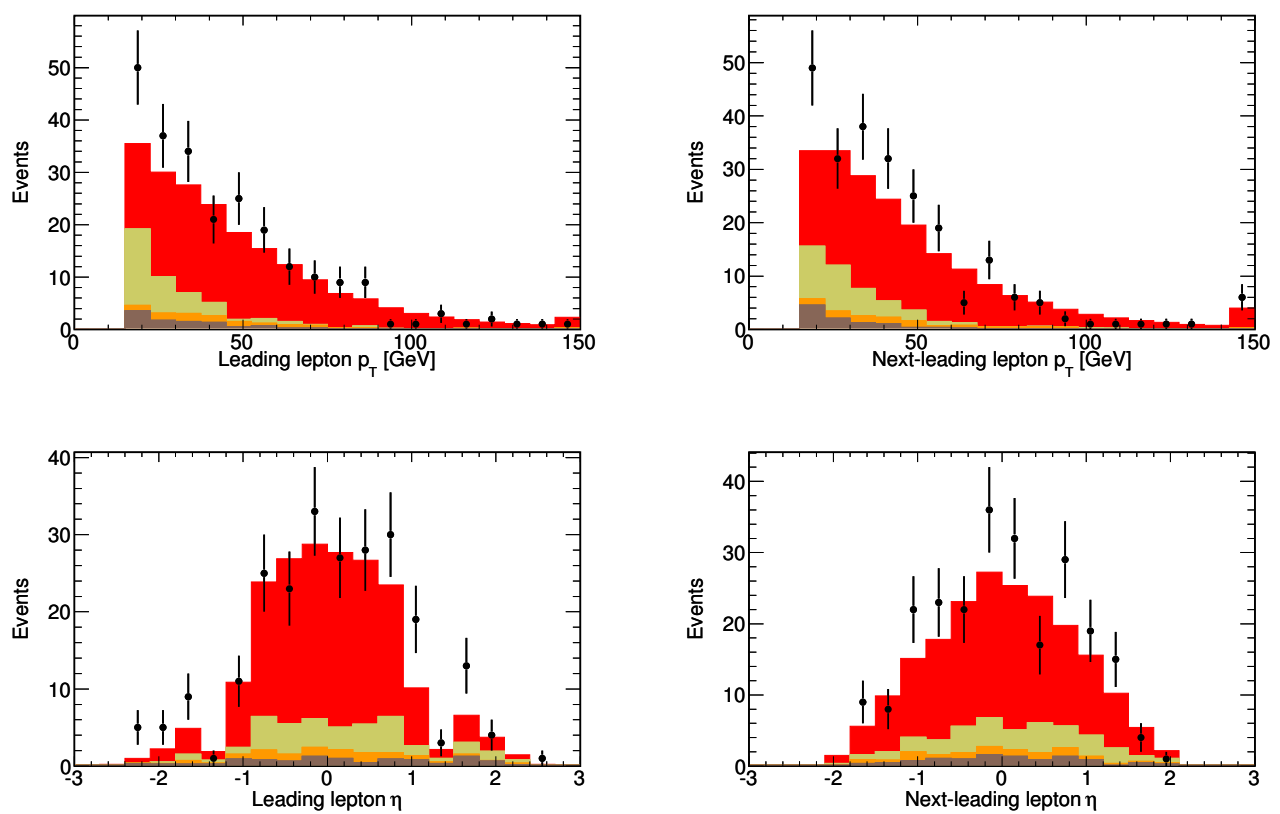

Figure A.34: $p_{T}$ and $\eta$ of the first and second leading lepton in the Run-IIb e $\mu$ channel. After requiring two leptons and two jets. 

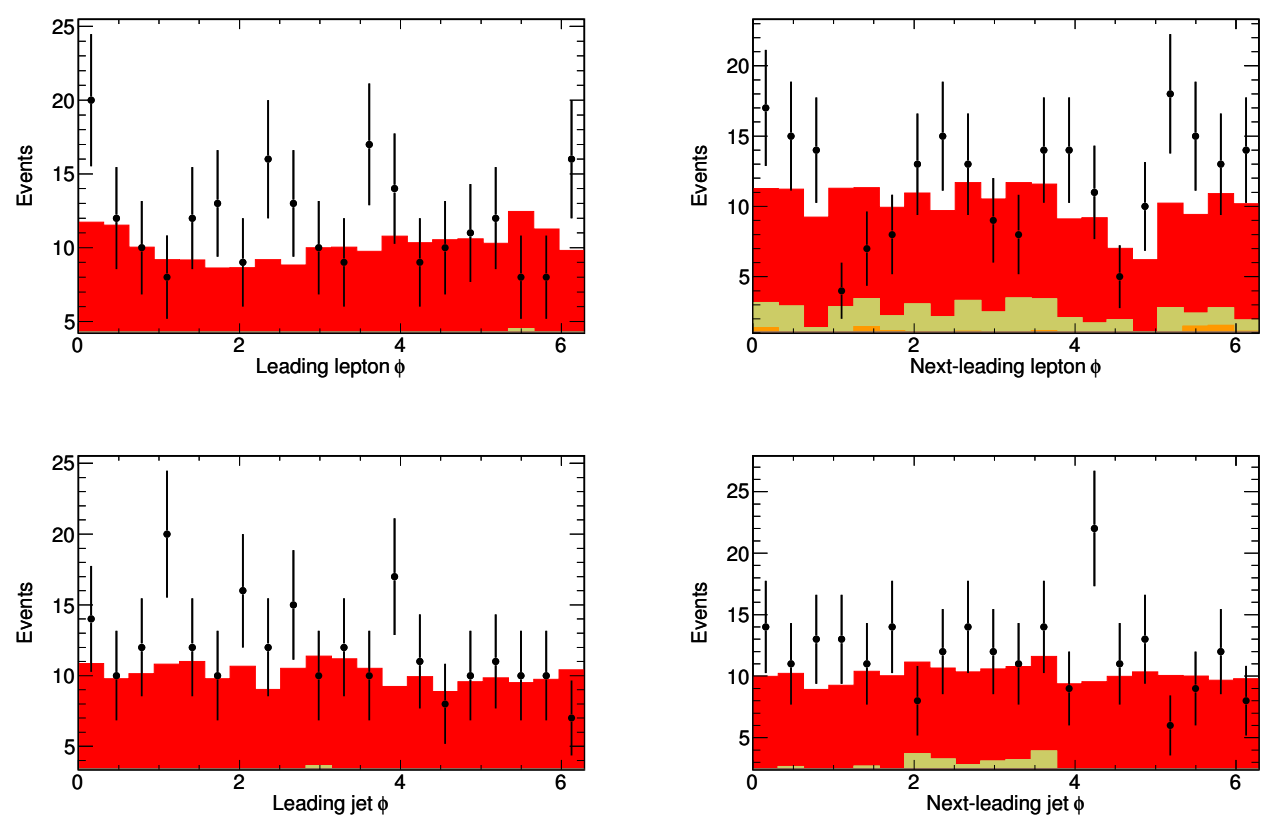

Figure A.35: $\phi$ of the first and second leading lepton and jet in the Run-IIb $\mathrm{e} \mu$ channel. After requiring two leptons and two jets.
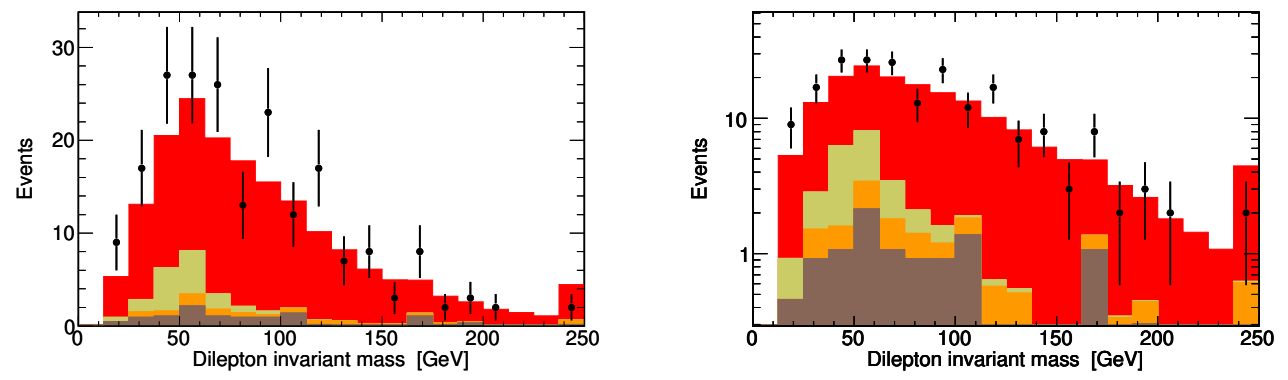

Figure A.36: Dilepton invariant mass spectrum in the Run-IIb e $\mu$ channel. After requiring two leptons and two jets. 

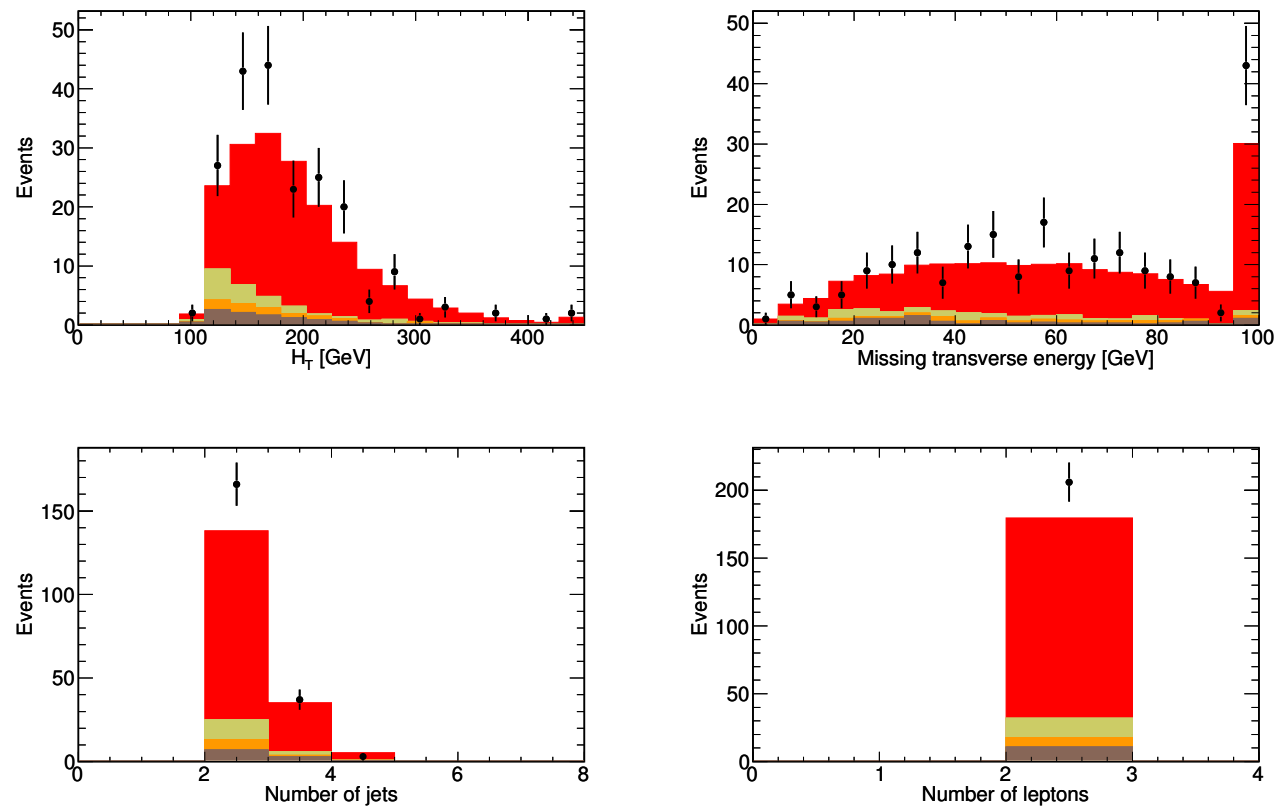

Figure A.37: $H_{T}$, missing transverse energy, number of jets and number of leptons in the Run-IIb e $\mu$ channel. After the final selection.
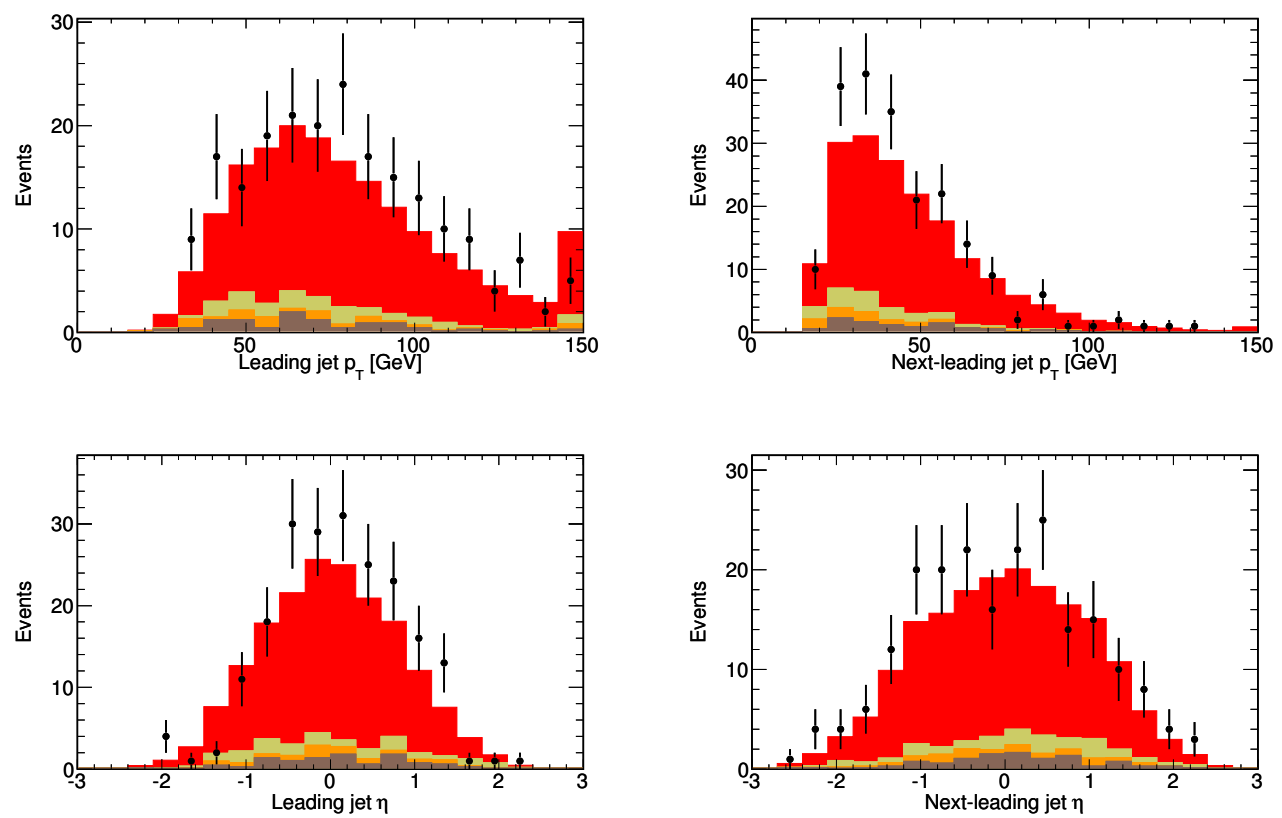

Figure A.38: $p_{T}$ and $\eta$ of the first and second leading jet in the Run-IIb e $\mu$ channel. After the final selection. 

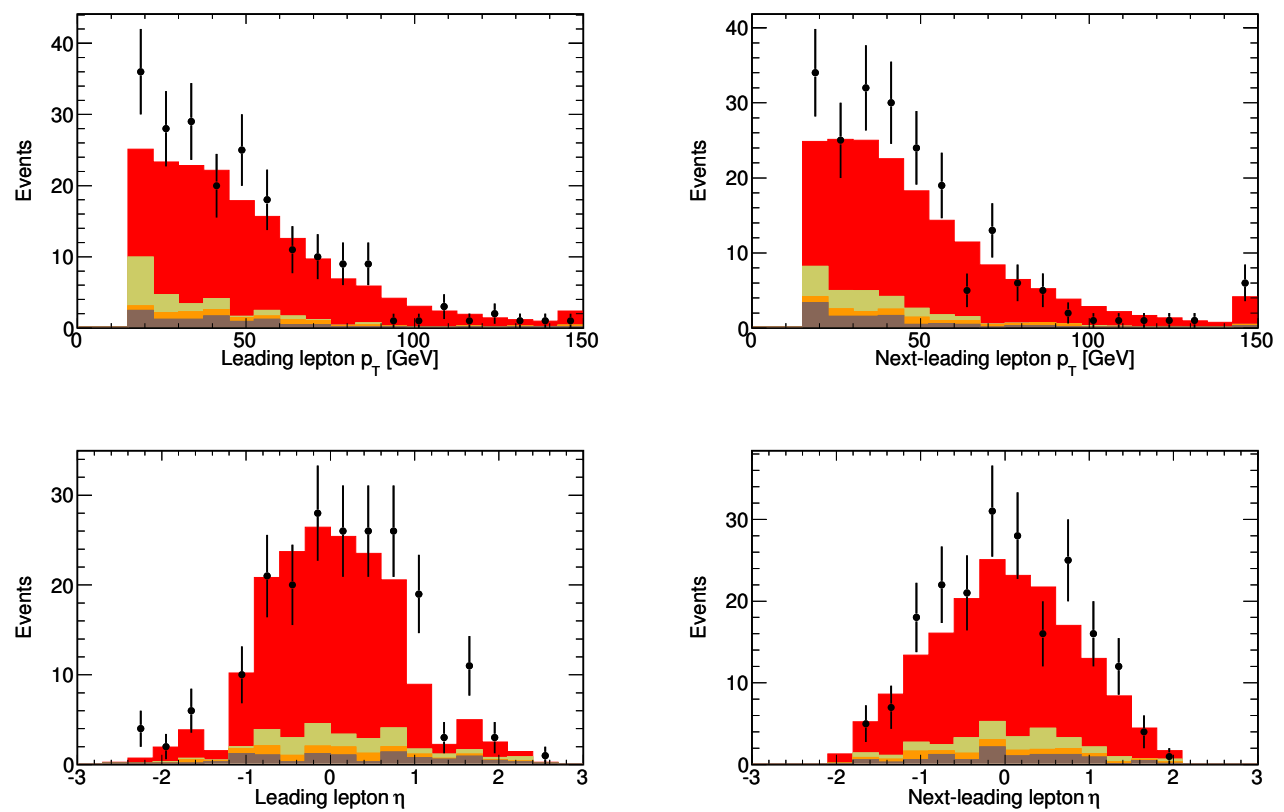

Figure A.39: $p_{T}$ and $\eta$ of the first and second leading lepton in the Run-IIb e $\mu$ channel. After the final selection.
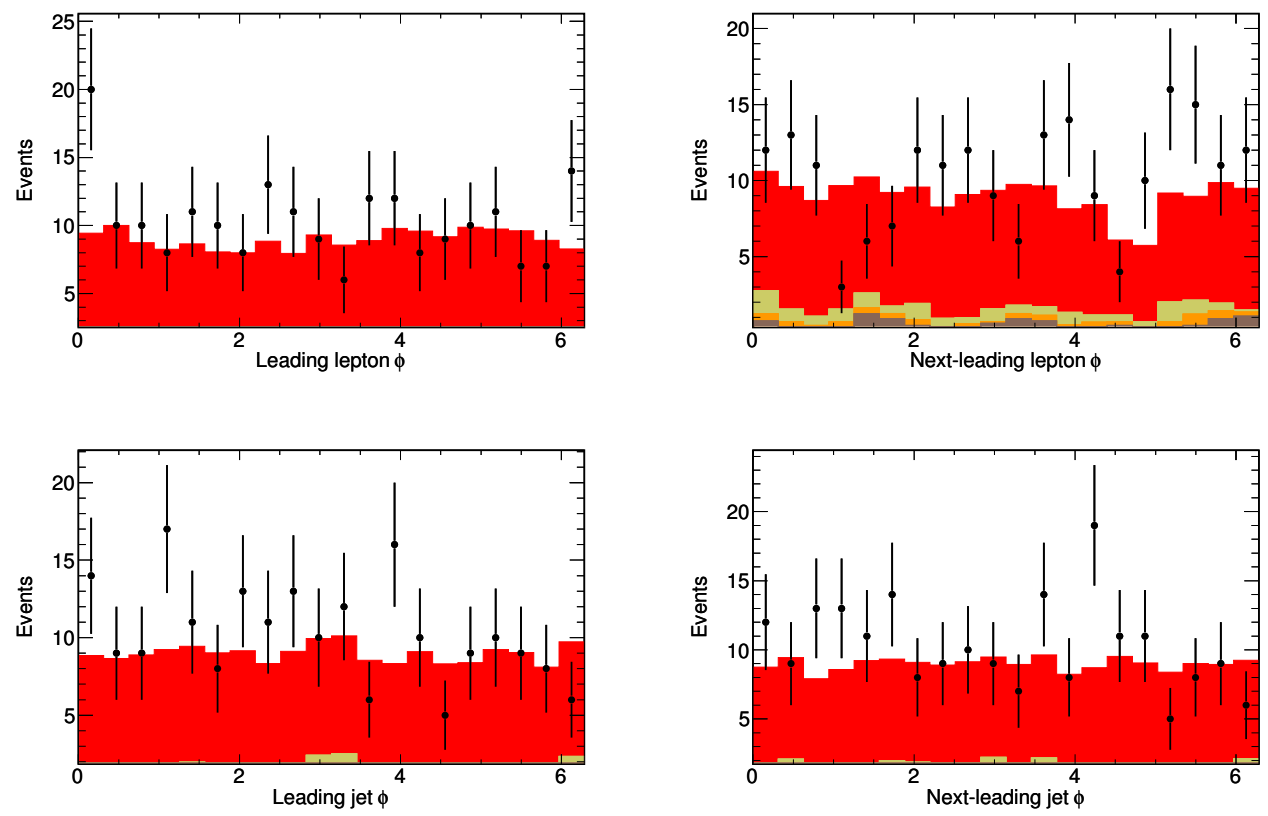

Figure A.40: $\phi$ of the first and second leading lepton and jet in the Run-IIb e $\mu$ channel. After the final selection. 

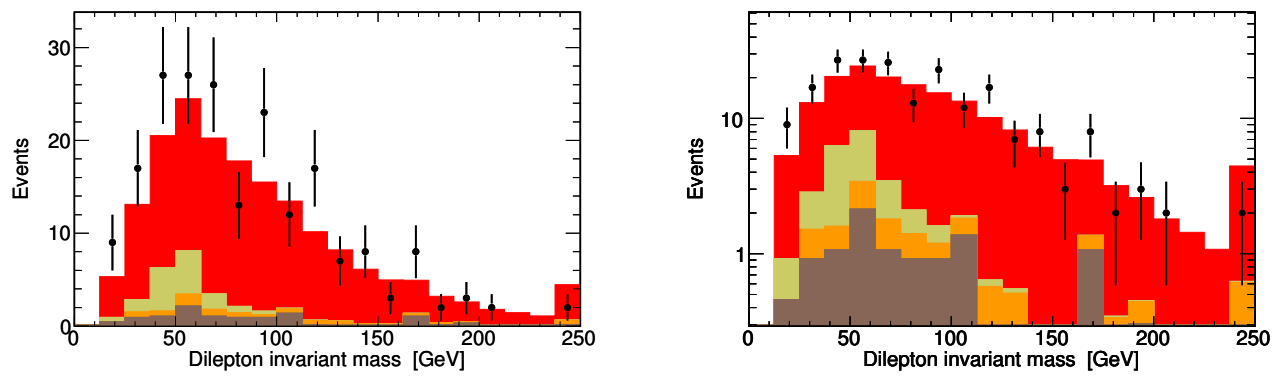

Figure A.41: Dilepton invariant mass spectrum in the Run-IIb e $\mu$ channel. After the final selection. 


\section{A.5 Run-IIa dimuon channel}
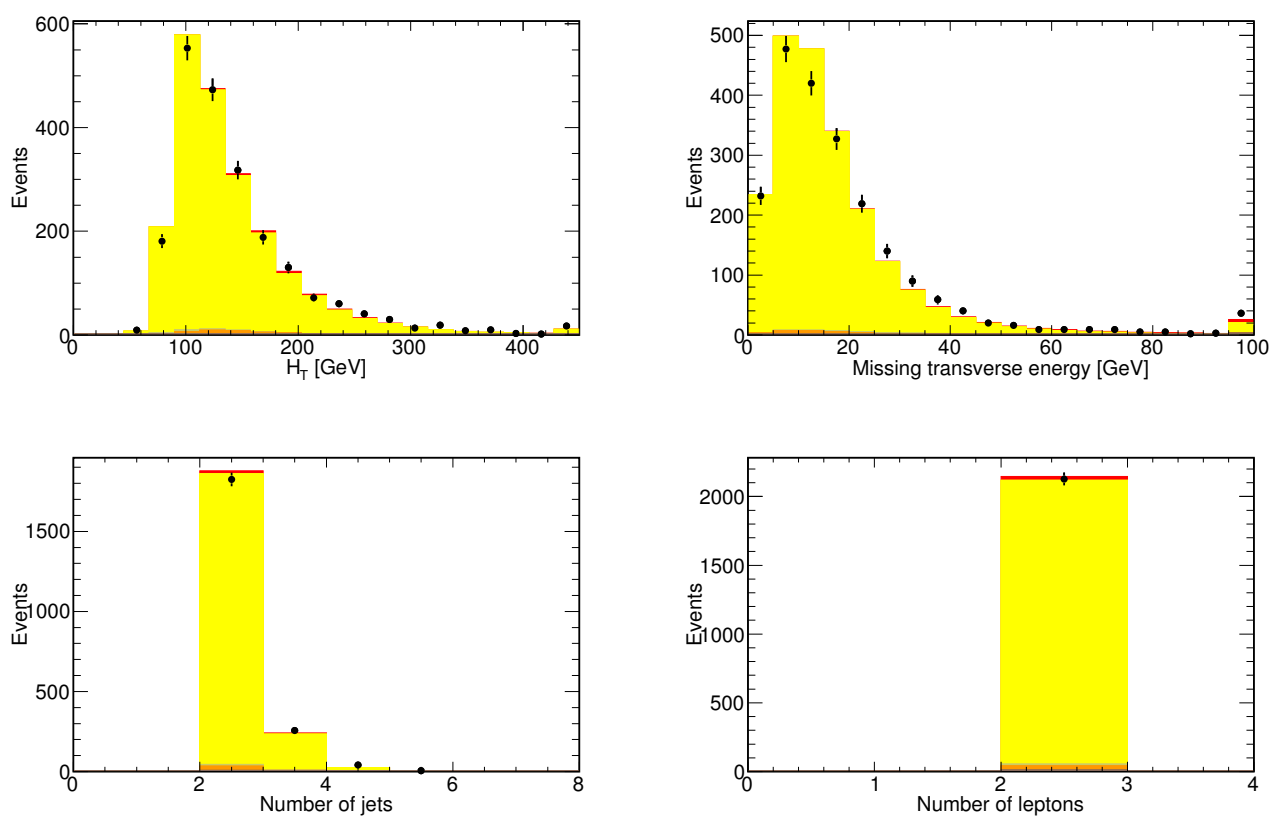

Figure A.42: $H_{T}$, missing transverse energy, number of jets and number of leptons in the Run-IIa $\mu \mu$ channel. After requiring two leptons and two jets. 

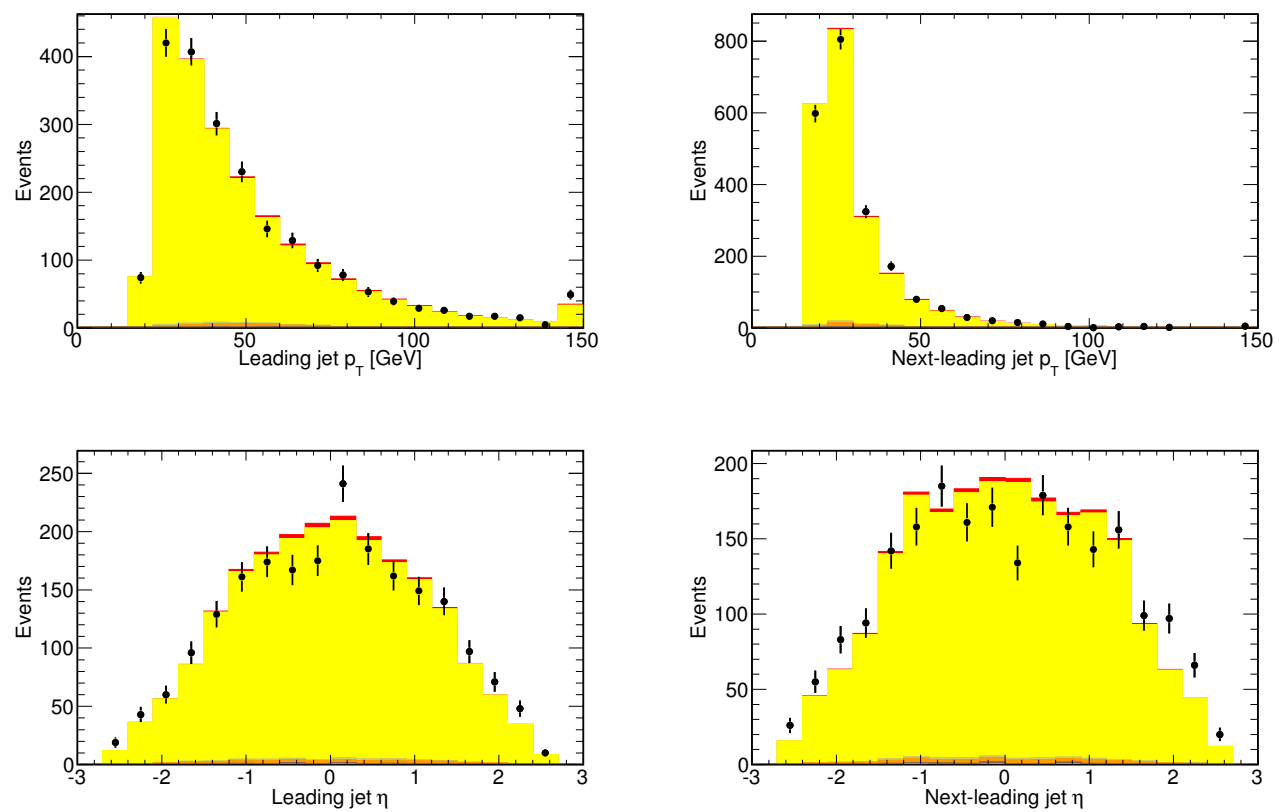

Figure A.43: $p_{T}$ and $\eta$ of the first and second leading jet in the Run-IIa $\mu \mu$ channel. After requiring two leptons and two jets.
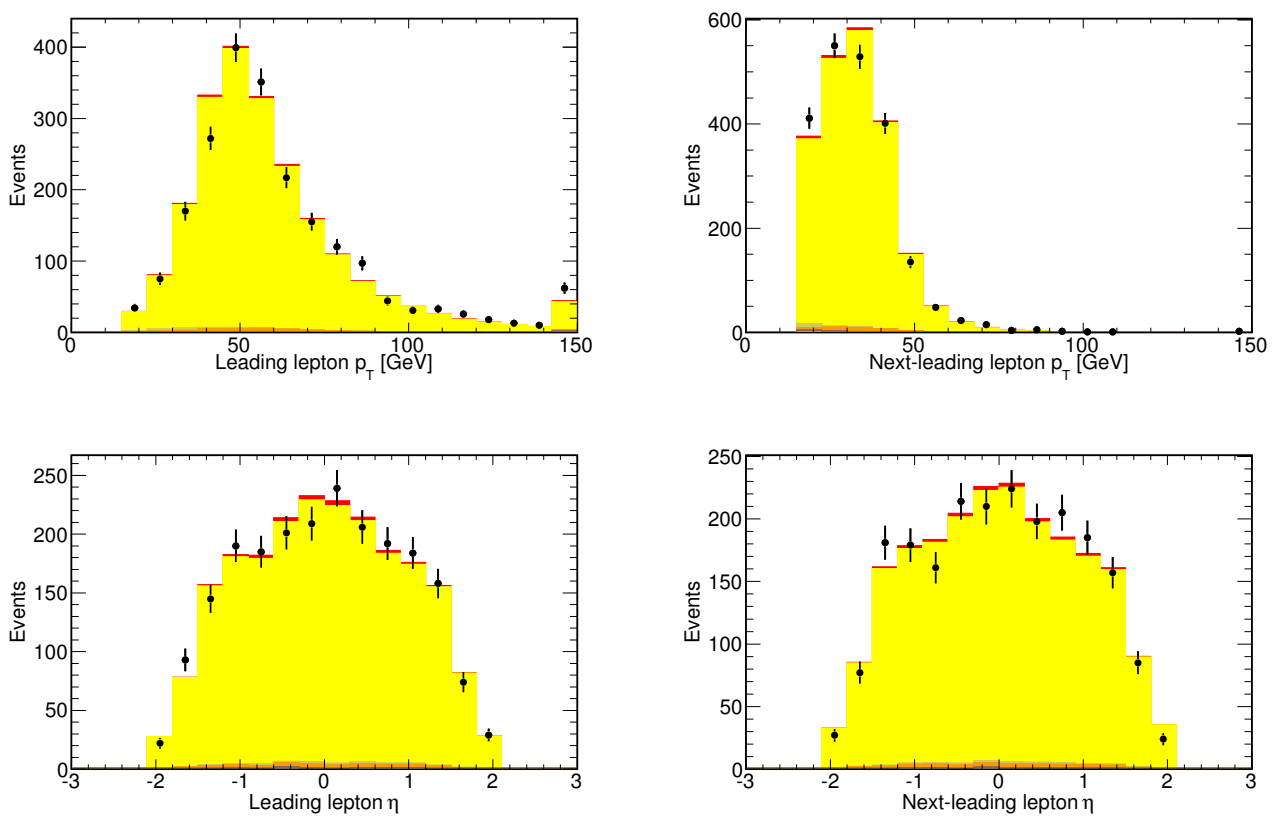

Figure A.44: $p_{T}$ and $\eta$ of the first and second leading lepton in the Run-IIa $\mu \mu$ channel. After requiring two leptons and two jets. 

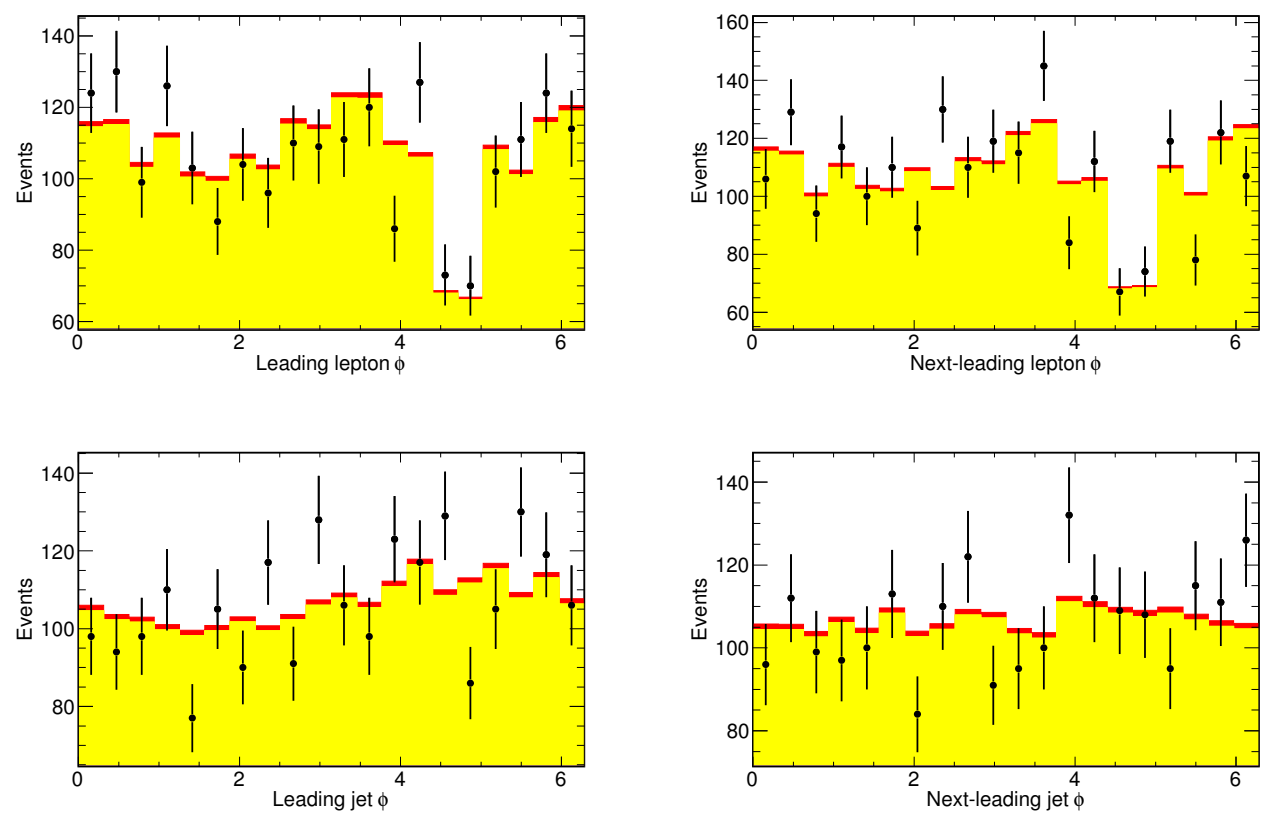

Figure A.45: $\phi$ of the first and second leading lepton and jet in the Run-IIa $\mu \mu$ channel. After requiring two leptons and two jets.
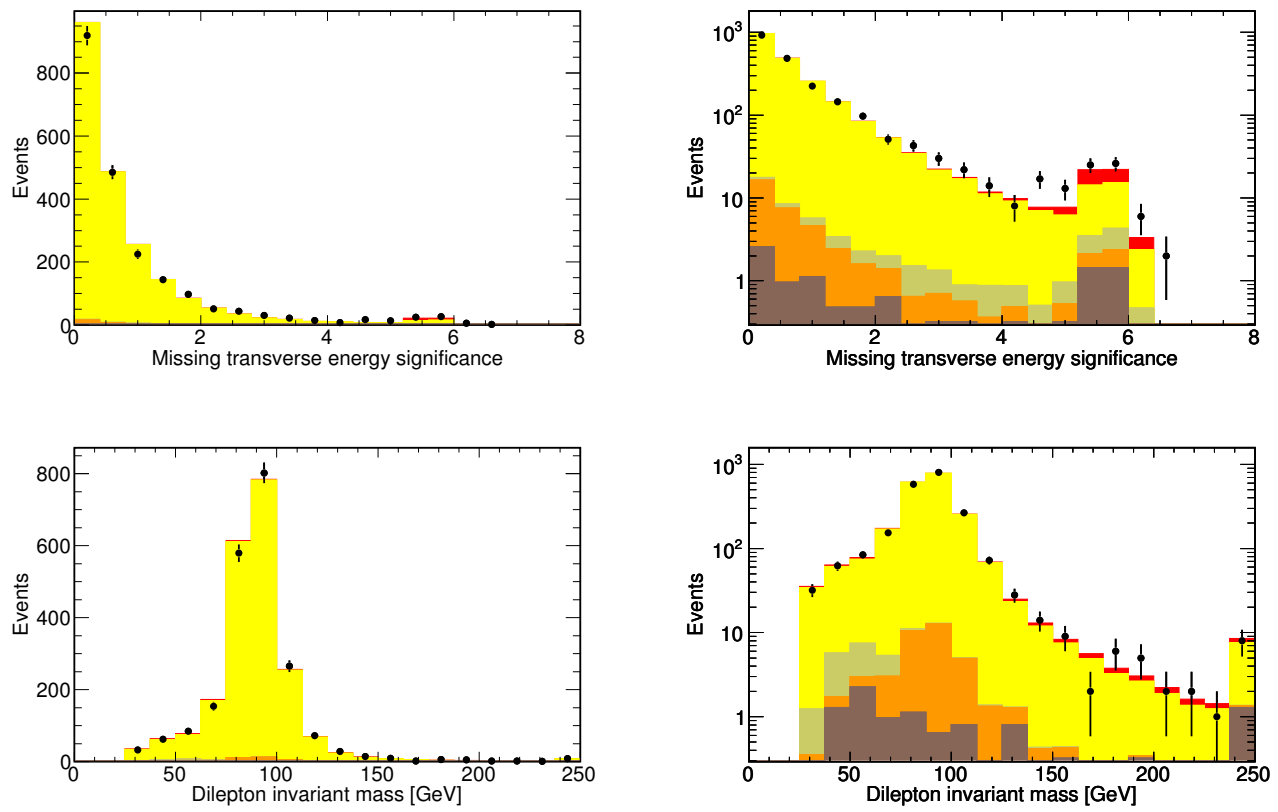

Figure A.46: $\mathbb{E}_{T}$ significance and dilepton invariant mass spectrum in the Run-IIa $\mu \mu$ channel. After requiring two leptons and two jets. 

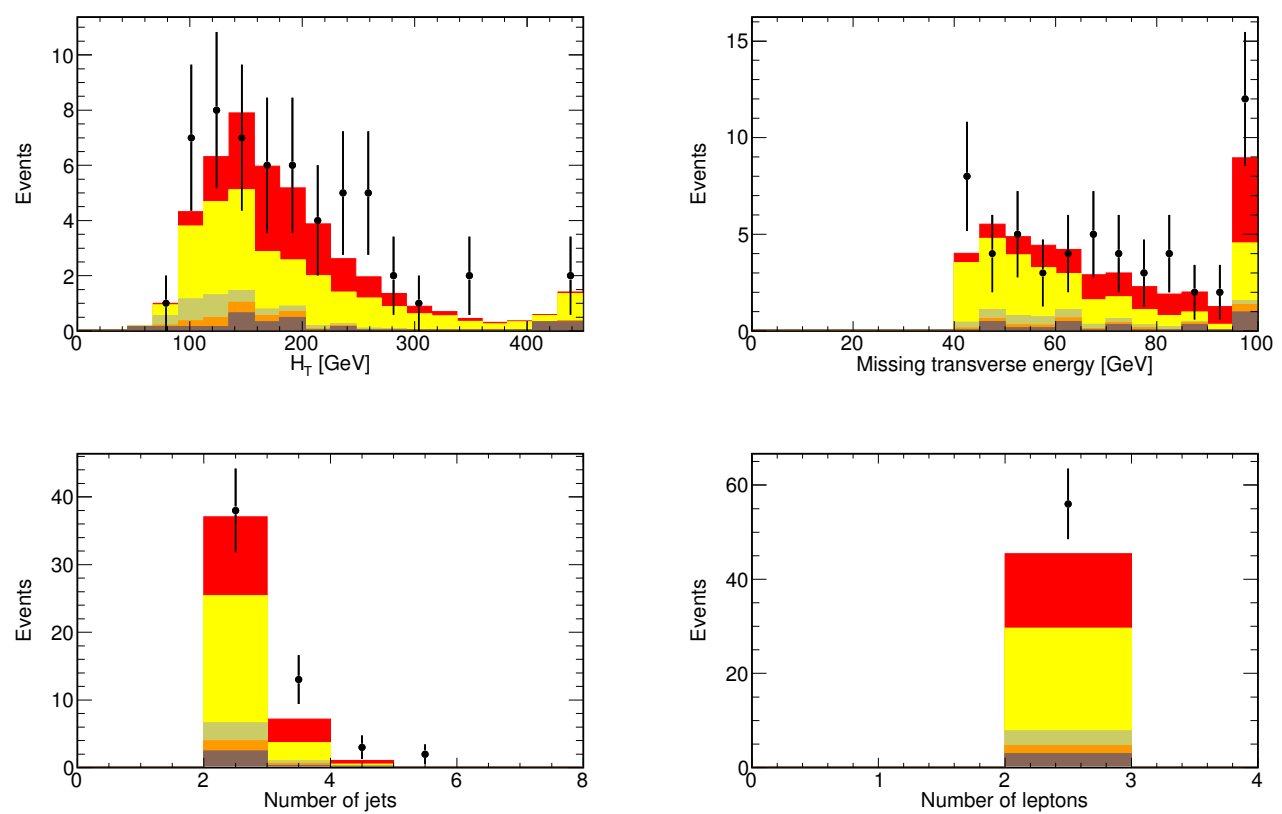

Figure A.47: $H_{T}$, missing transverse energy, number of jets and number of leptons in the Run-IIa $\mu \mu$ channel. After the final selection.
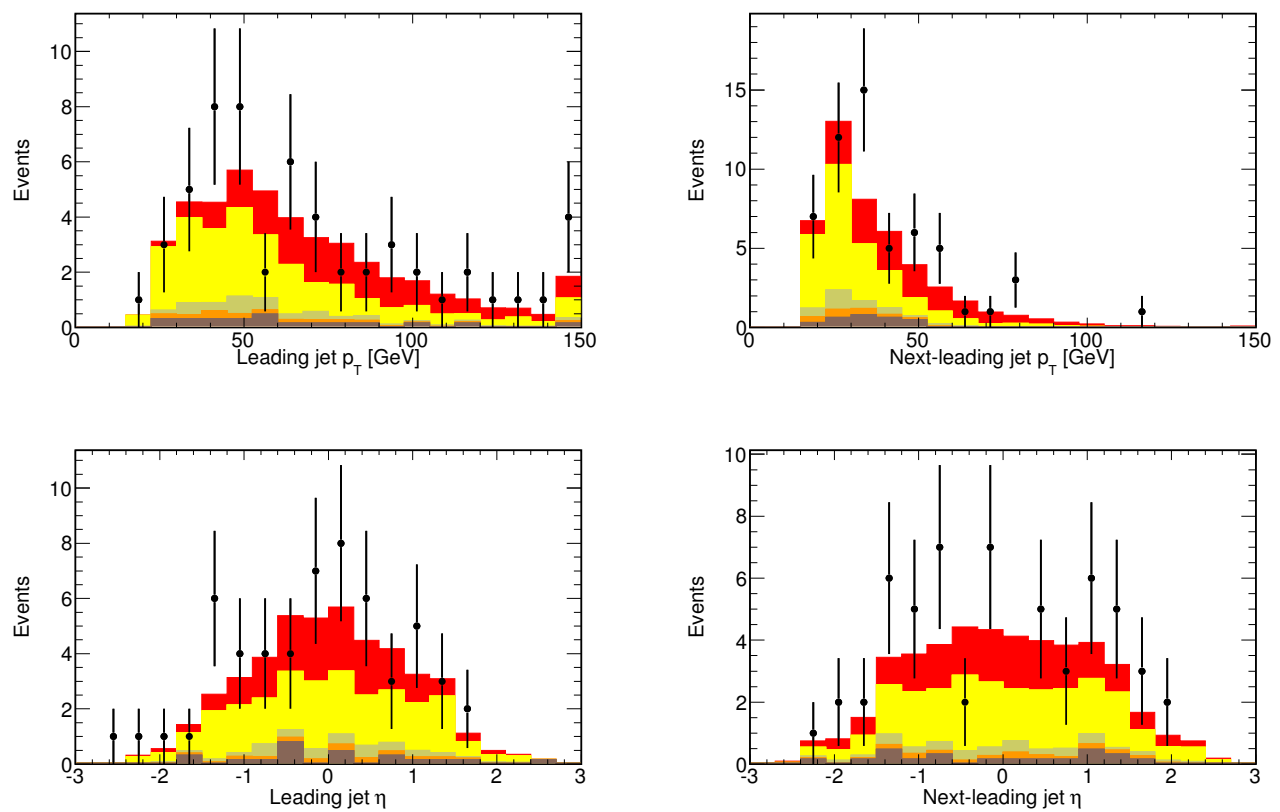

Figure A.48: $p_{T}$ and $\eta$ of the first and second leading jet in the Run-IIa $\mu \mu$ channel. After the final selection. 

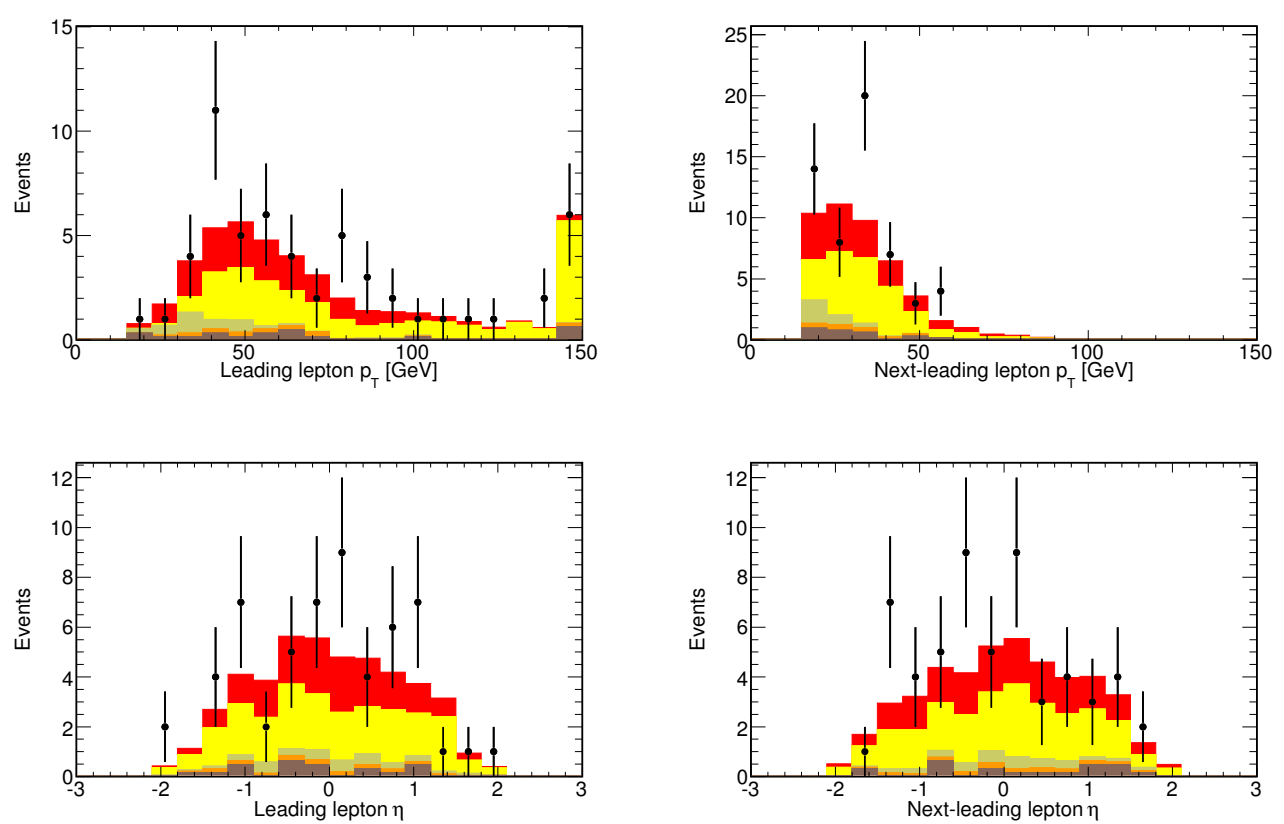

Figure A.49: $p_{T}$ and $\eta$ of the first and second leading lepton in the Run-IIa $\mu \mu$ channel. After the final selection.
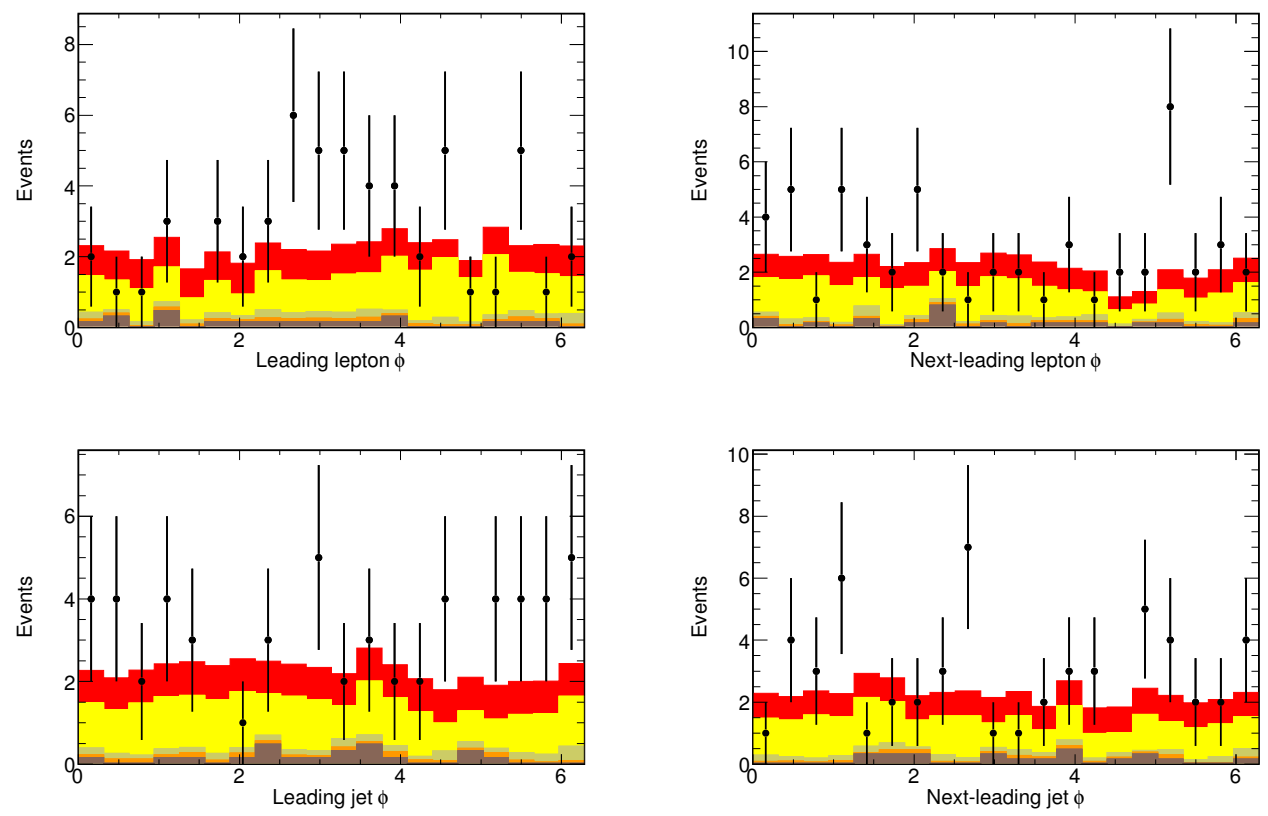

Figure A.50: $\phi$ of the first and second leading lepton and jet in the Run-IIa $\mu \mu$ channel. After the final selection. 

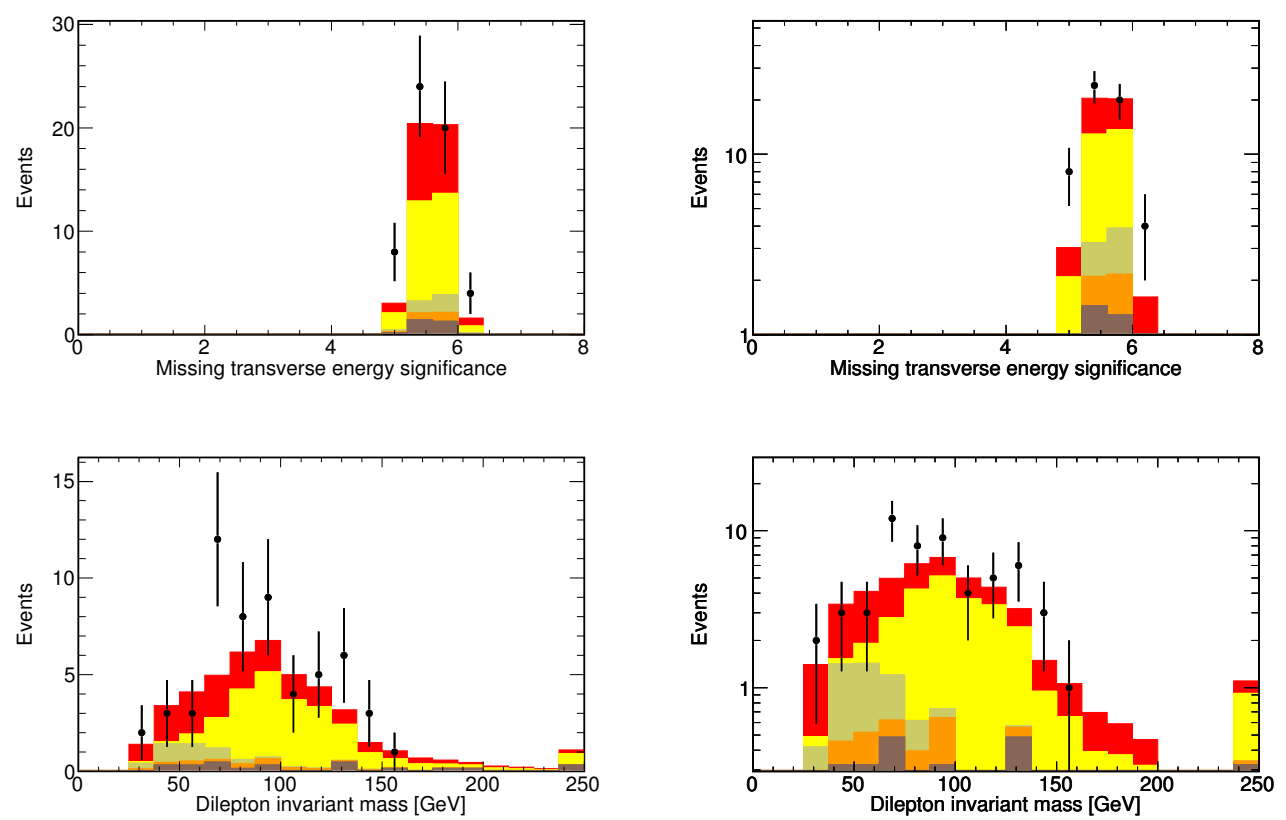

Figure A.51: met significance and dilepton invariant mass spectrum in the Run-IIa $\mu \mu$ channel. After the final selection. 


\section{A.6 Run-IIb dimuon channel}
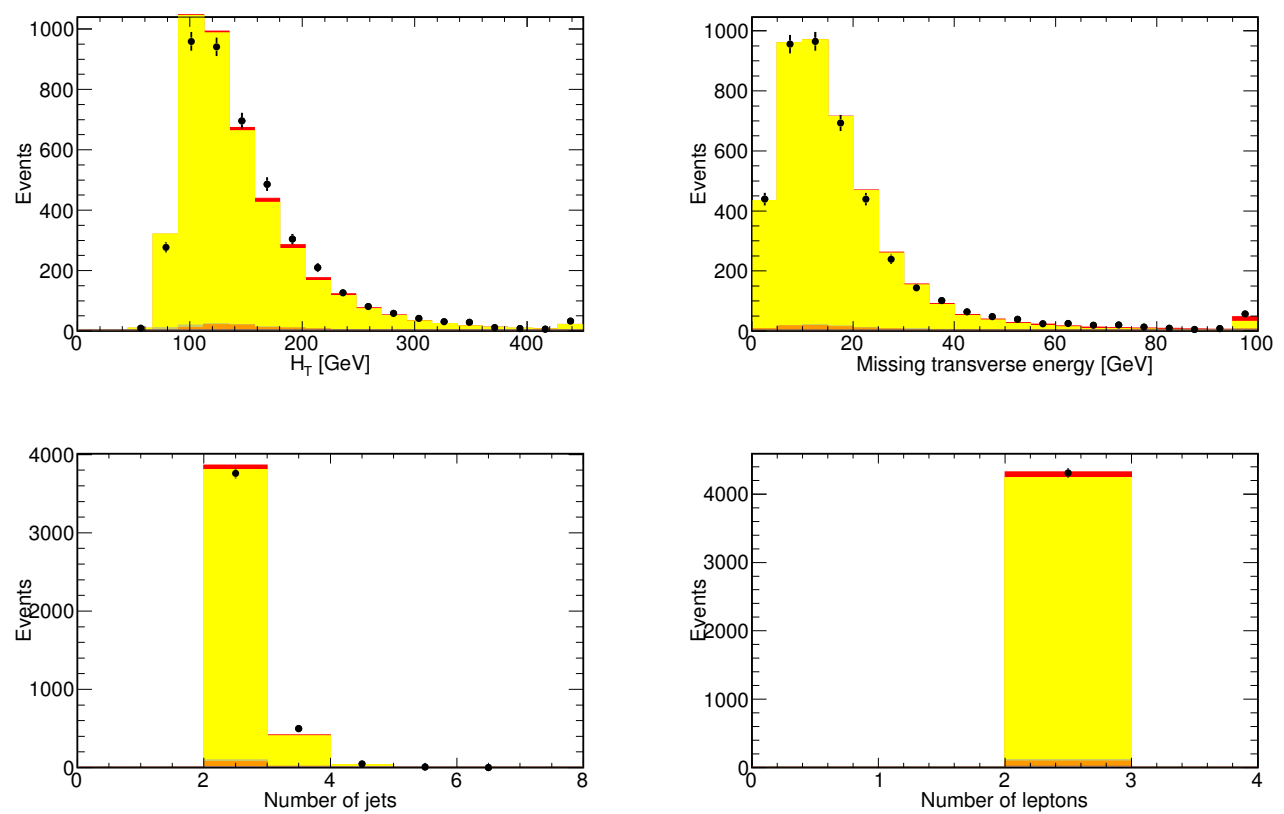

Figure A.52: $H_{T}$, missing transverse energy, number of jets and number of leptons in the Run-IIb $\mu \mu$ channel. After requiring two leptons and two jets. 

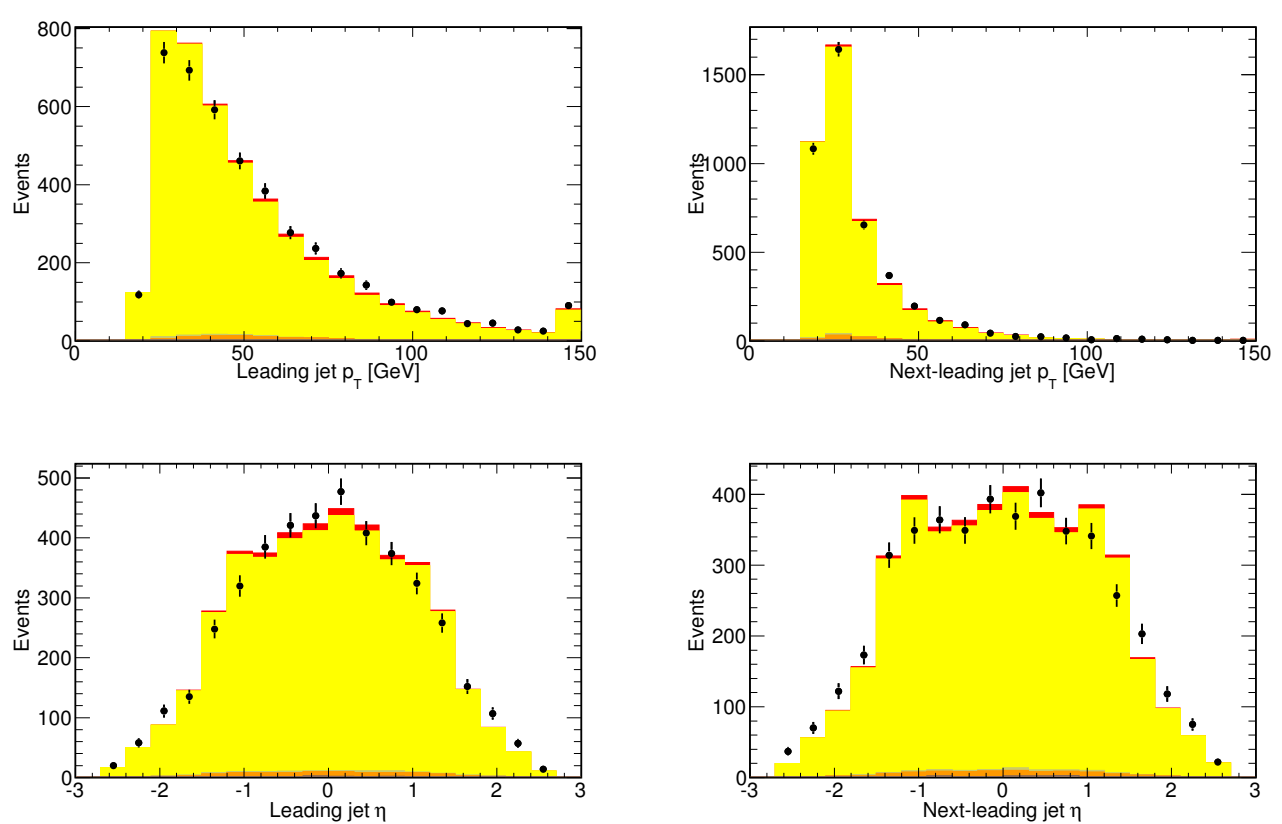

Figure A.53: $p_{T}$ and $\eta$ of the first and second leading jet in the Run-IIb $\mu \mu$ channel. After requiring two leptons and two jets.
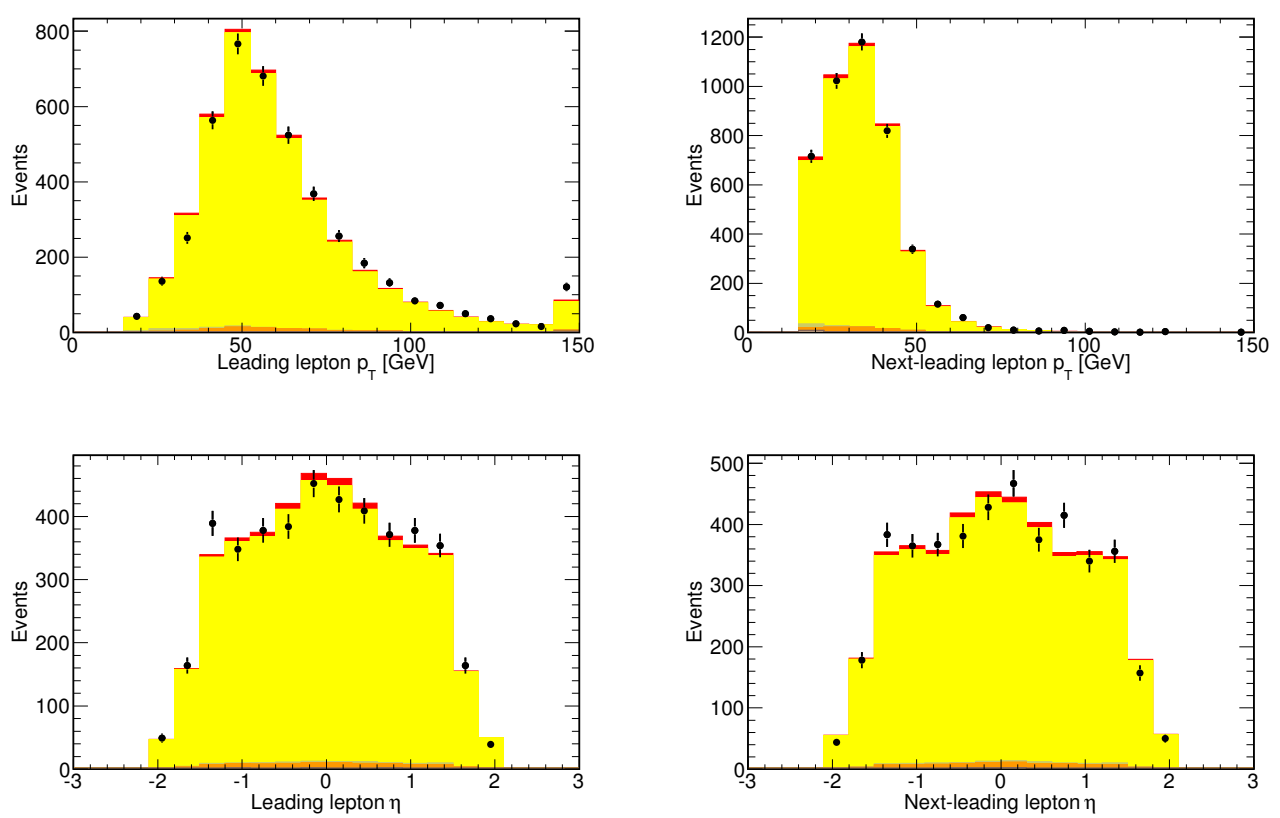

Figure A.54: $p_{T}$ and $\eta$ of the first and second leading lepton in the Run-IIb $\mu \mu$ channel. After requiring two leptons and two jets. 

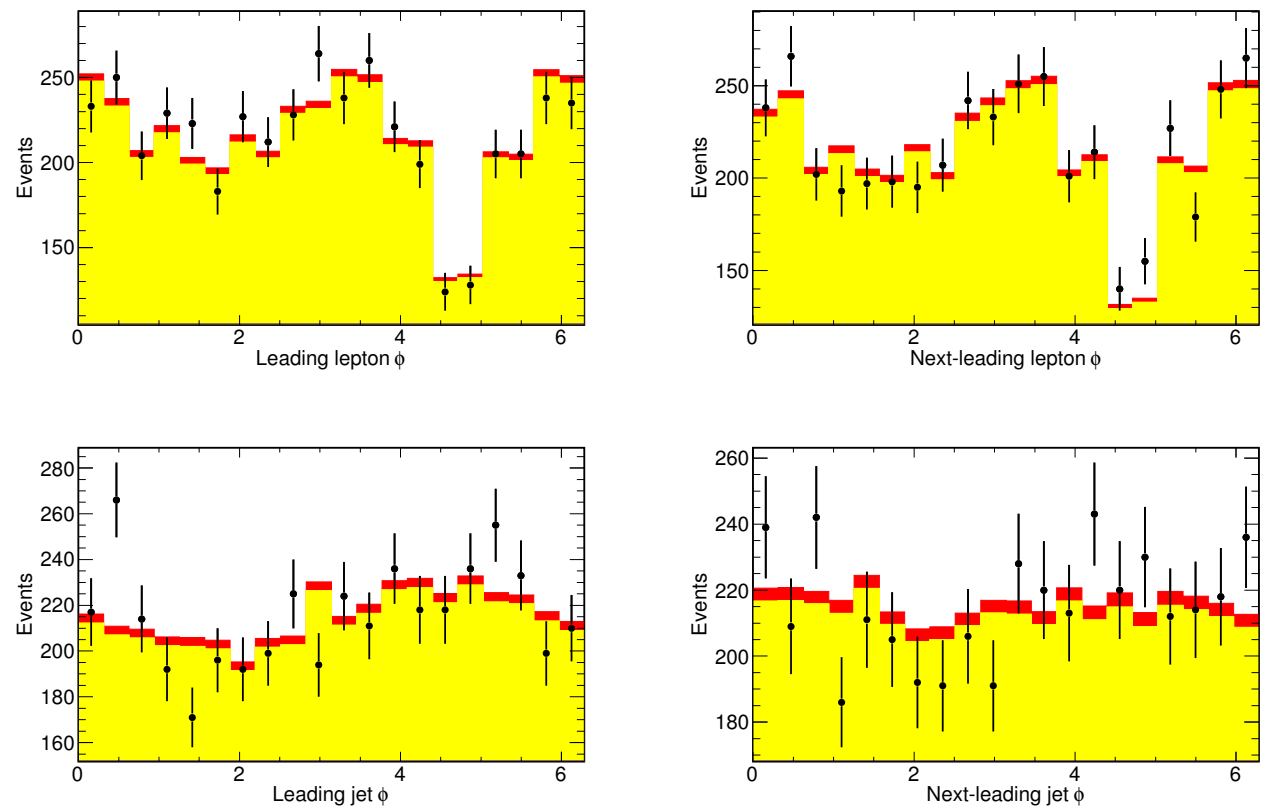

Figure A.55: $\phi$ of the first and second leading lepton and jet in the Run-IIb $\mu \mu$ channel. After requiring two leptons and two jets.
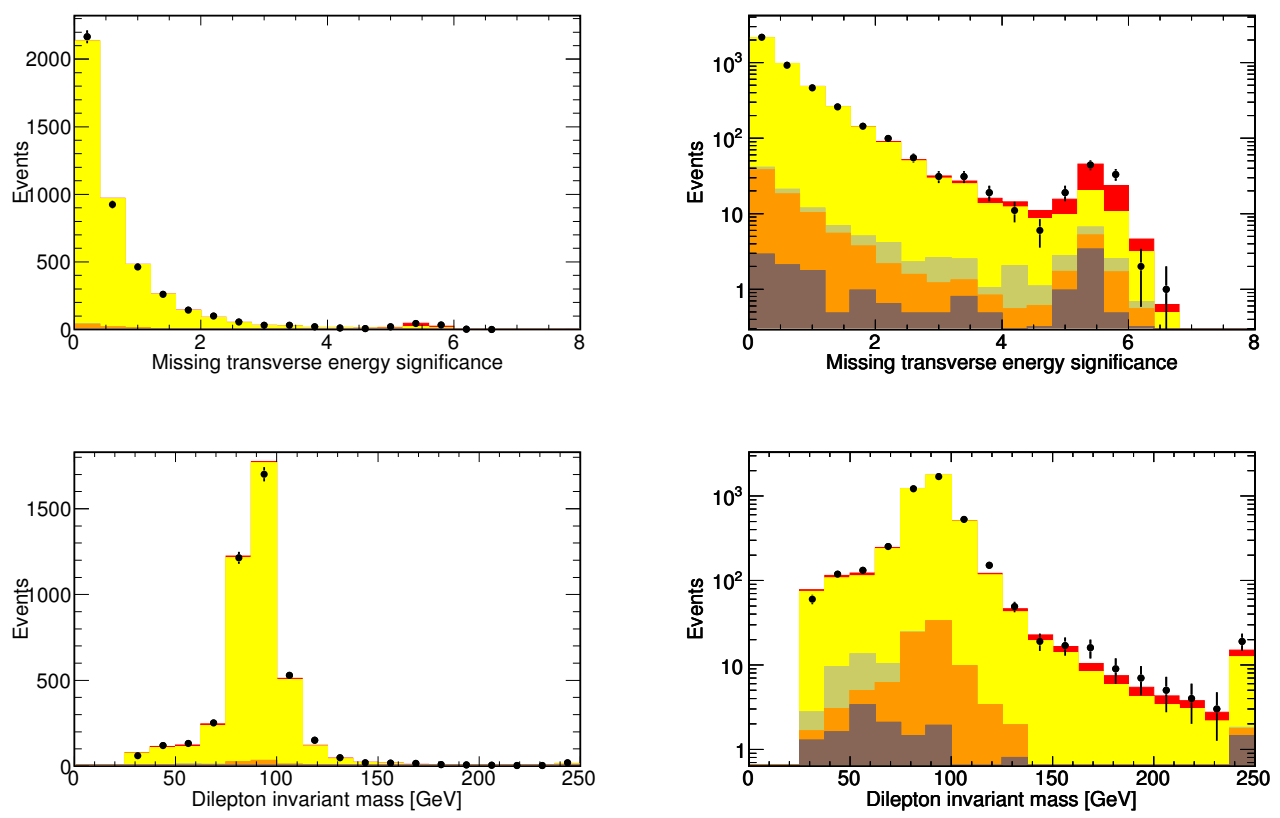

Figure A.56: $\mathbb{E}_{T}$ significance and dilepton invariant mass spectrum in the Run-IIb $\mu \mu$ channel. After requiring two leptons and two jets. 

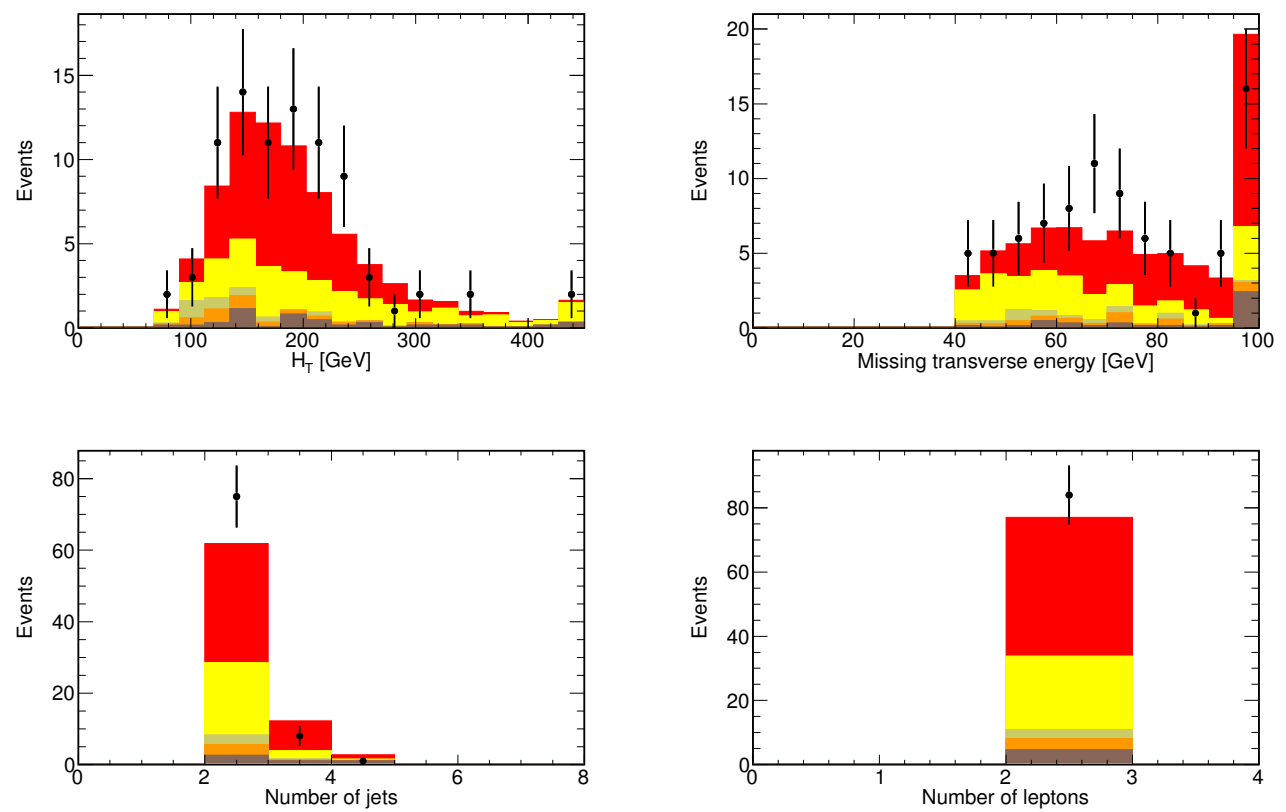

Figure A.57: $H_{T}$, missing transverse energy, number of jets and number of leptons in the Run-IIb $\mu \mu$ channel. After the final selection.
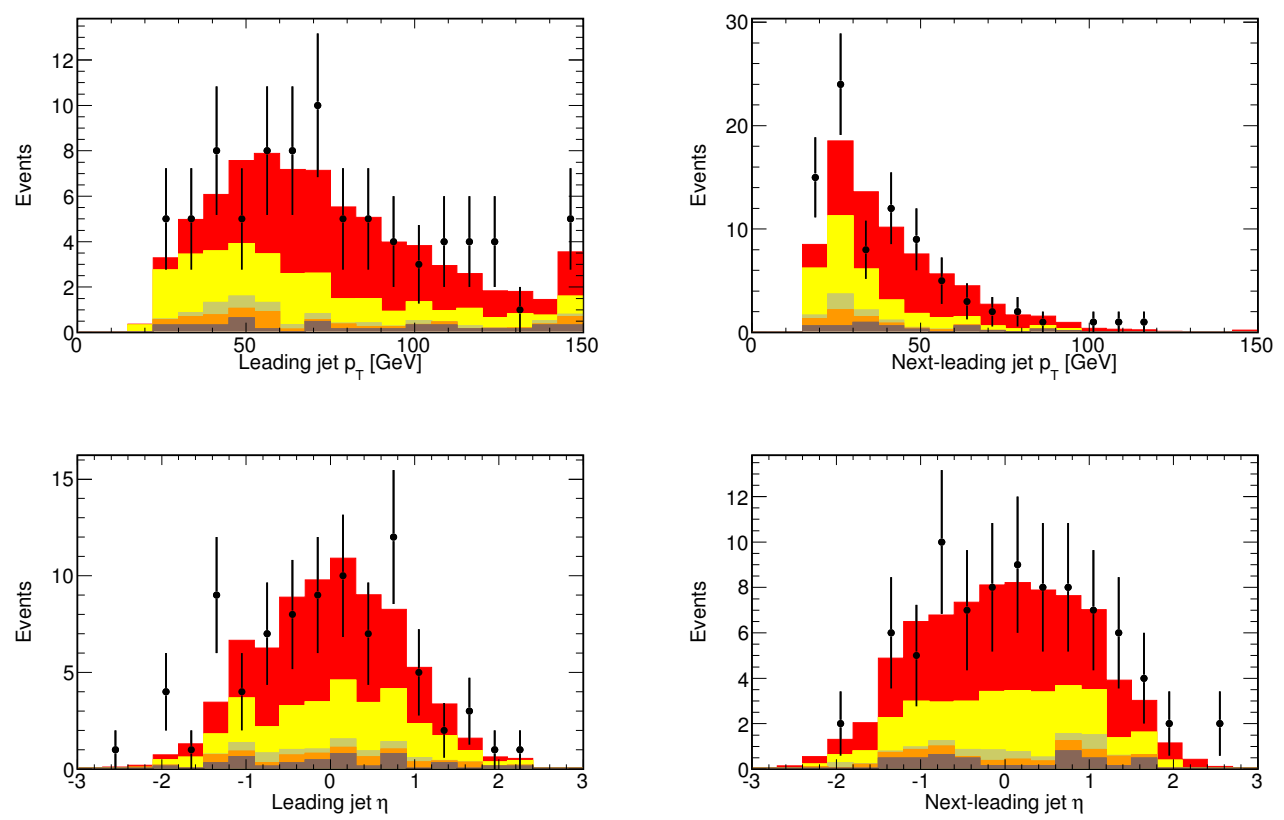

Figure A.58: $p_{T}$ and $\eta$ of the first and second leading jet in the Run-IIb $\mu \mu$ channel. After the final selection. 

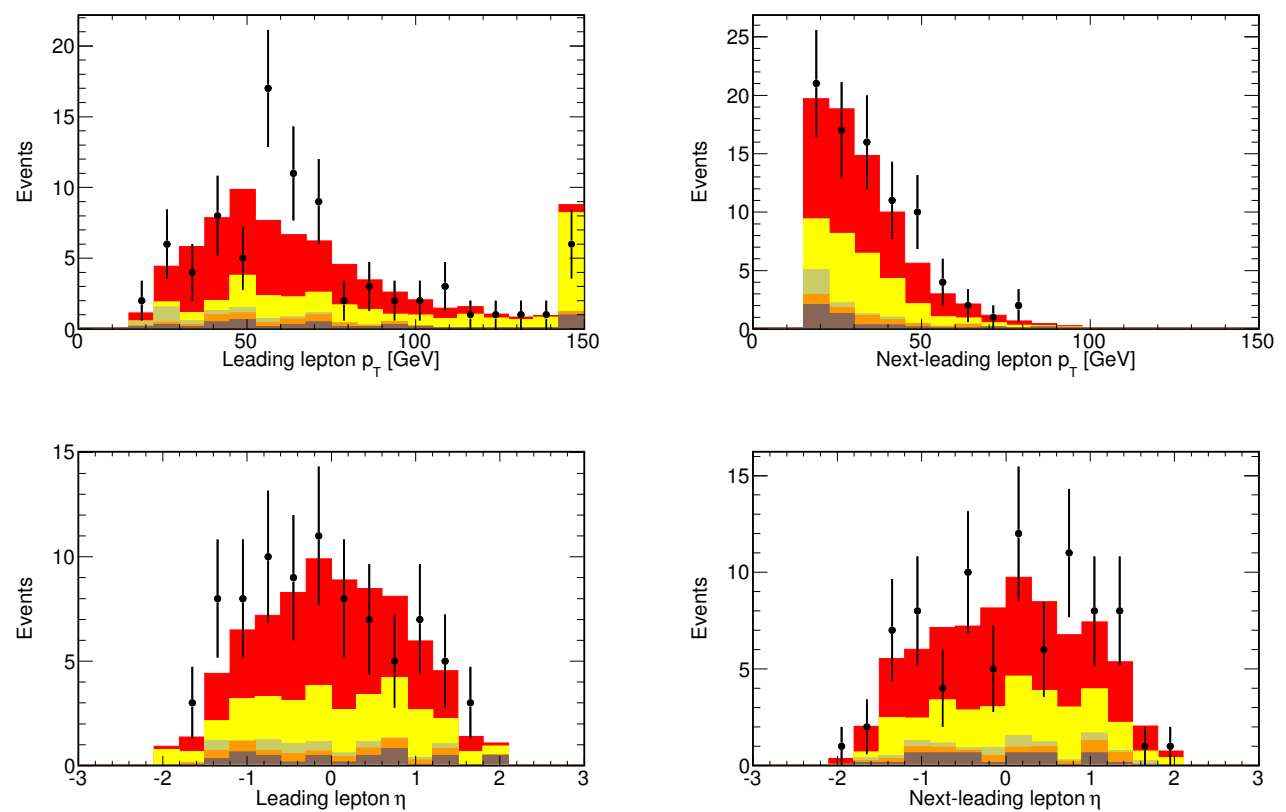

Figure A.59: $p_{T}$ and $\eta$ of the first and second leading lepton in the Run-IIb $\mu \mu$ channel. After the final selection.
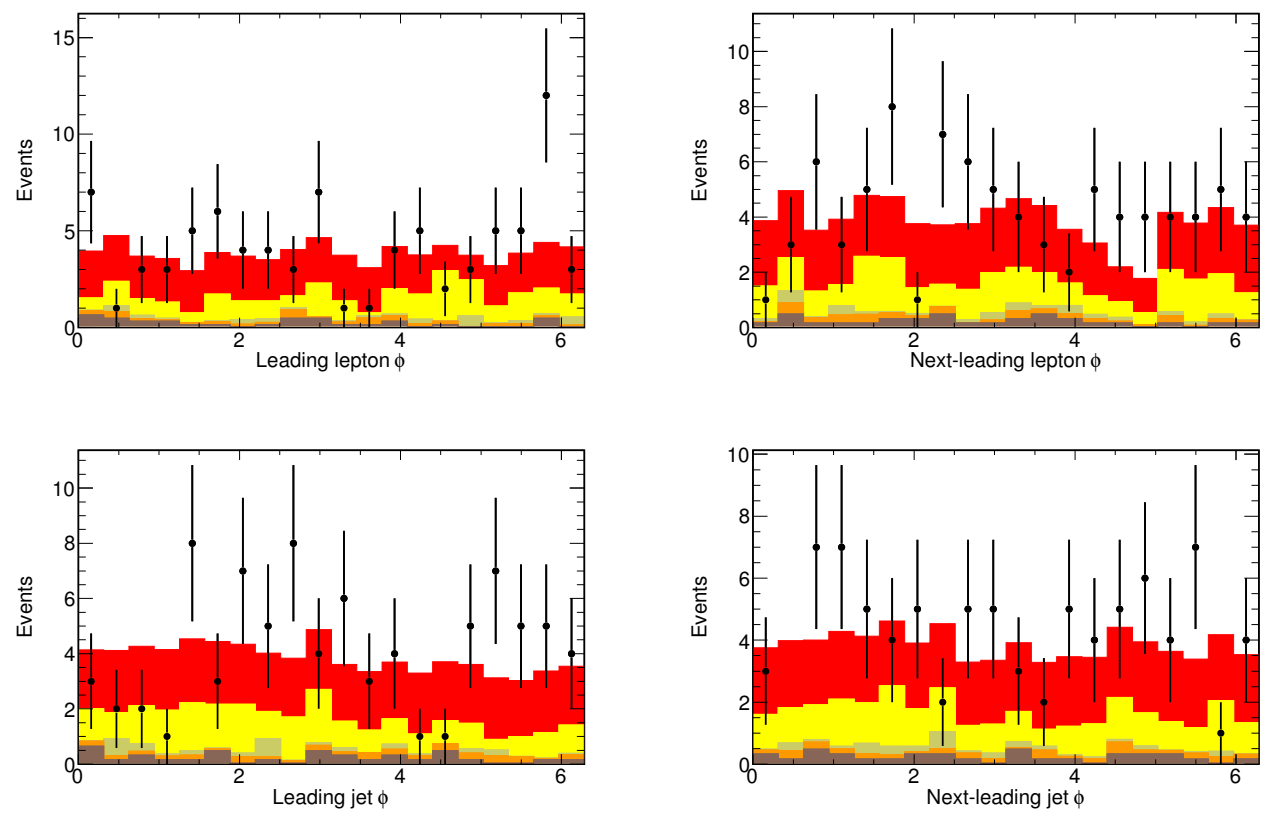

Figure A.60: $\phi$ of the first and second leading lepton and jet in the Run-IIb $\mu \mu$ channel. After the final selection. 

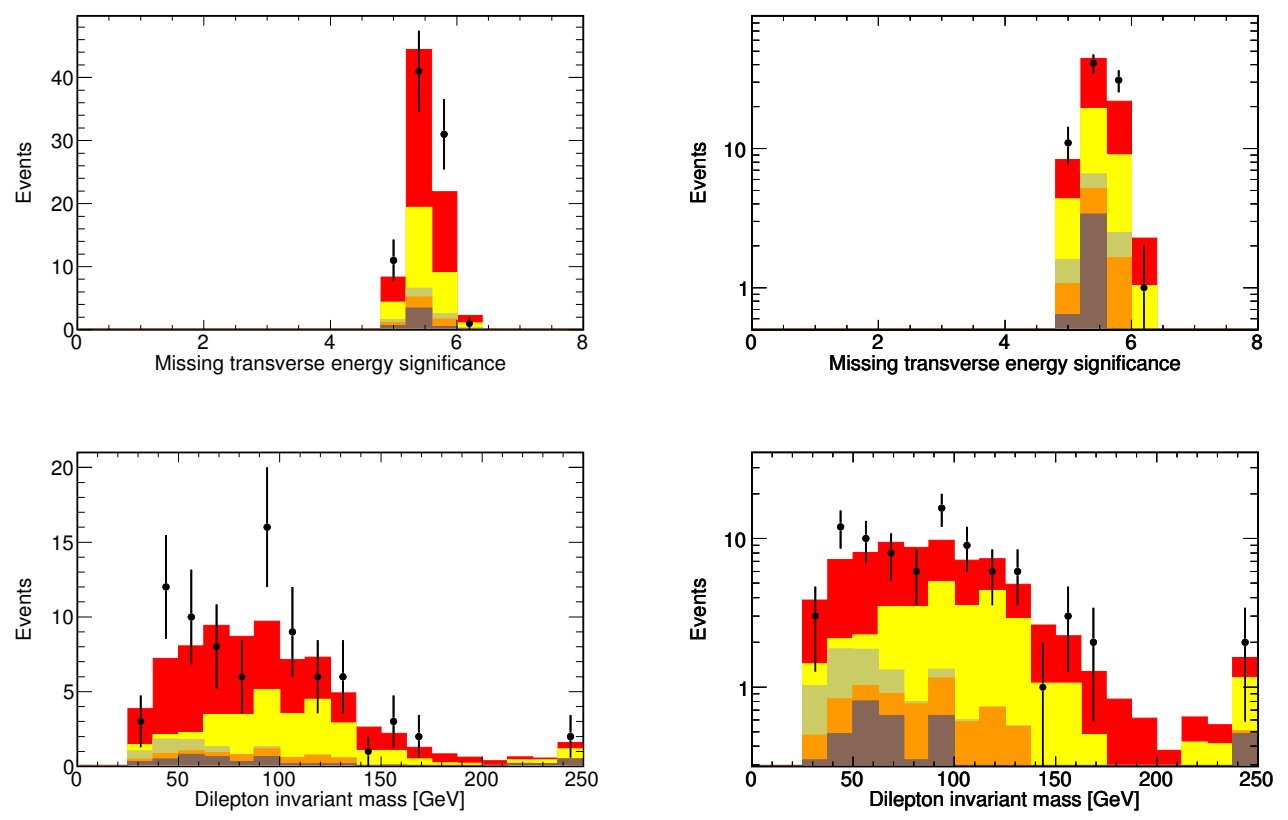

Figure A.61: $\mathbb{E}_{T}$ significance and dilepton invariant mass spectrum in the Run-IIb $\mu \mu$ channel. After the final selection. 


\title{
Appendix B
}

\section{Electron identification variables}

\author{
B.1 Central calorimeter variables
}
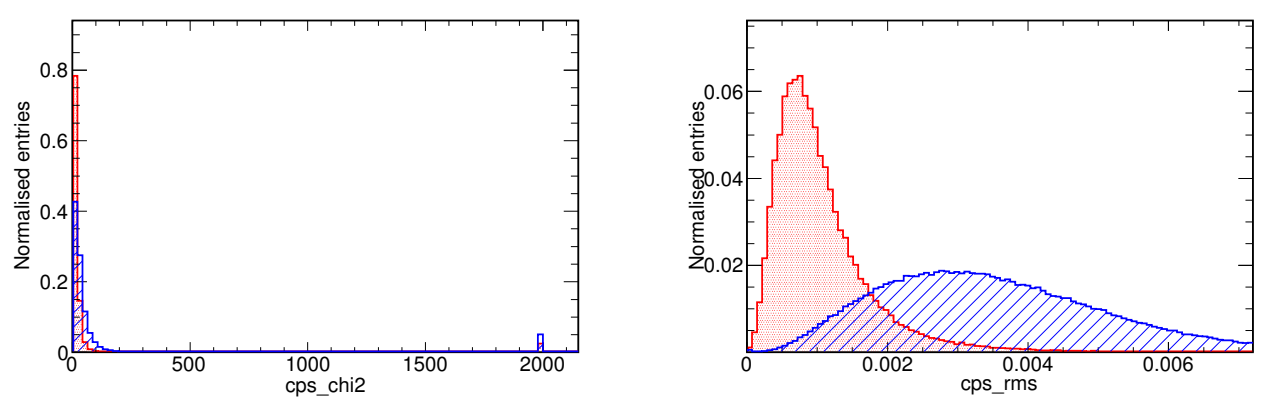

Figure B.1: In red the distribution for "real" and in blue for "fake" electrons. 

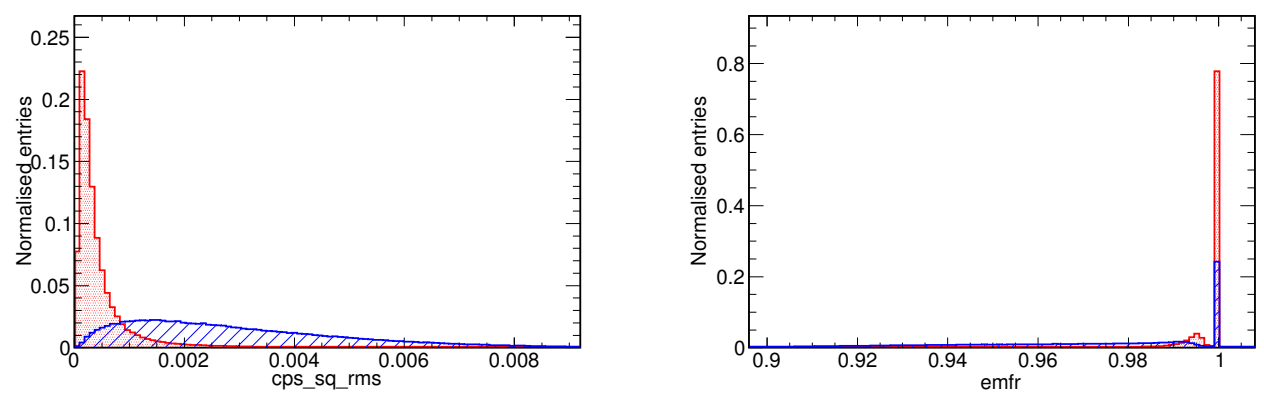

Figure B.2: In red the distribution for "real" and in blue for "fake" electrons.
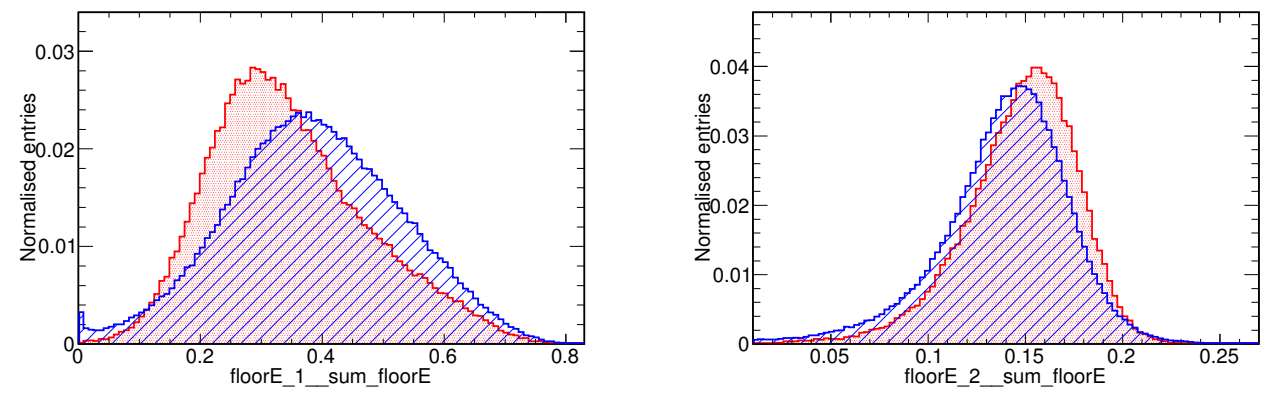

Figure B.3: In red the distribution for "real" and in blue for "fake" electrons.
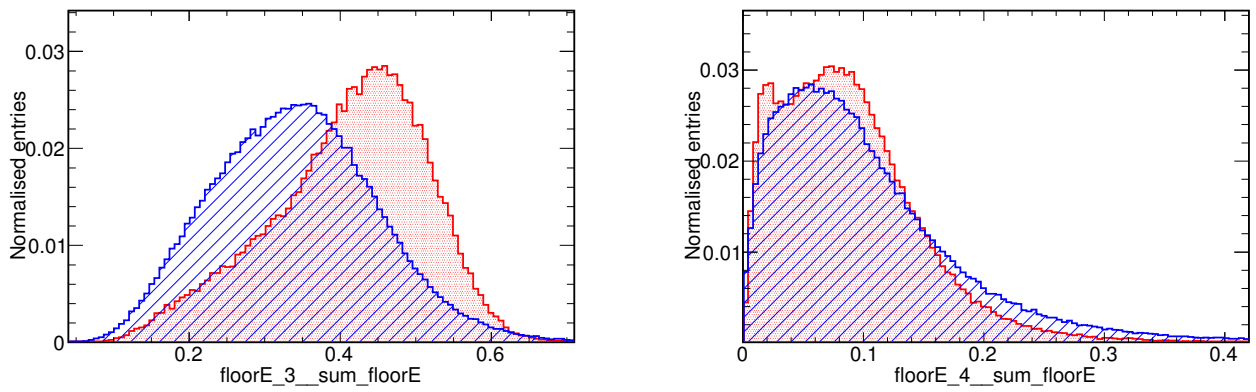

Figure B.4: In red the distribution for "real" and in blue for "fake" electrons. 

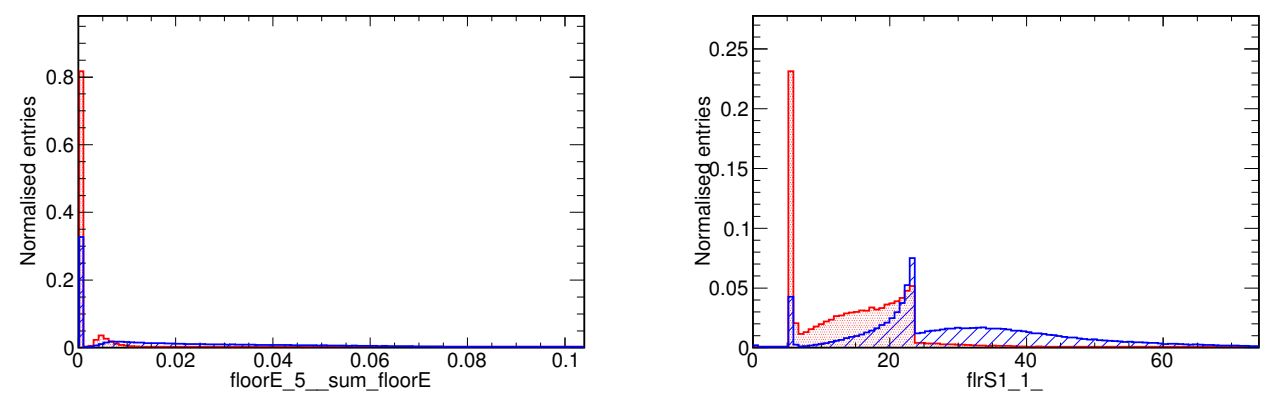

Figure B.5: In red the distribution for "real" and in blue for "fake" electrons.
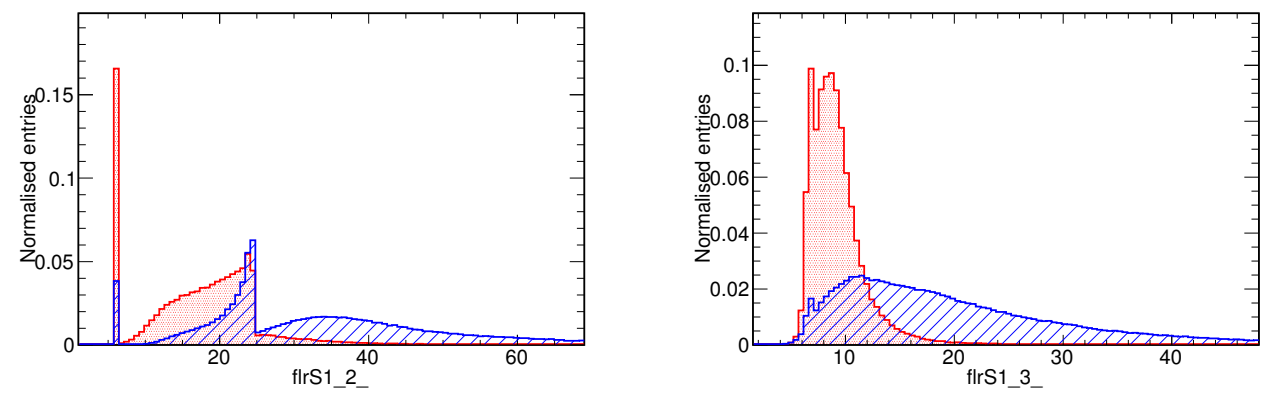

Figure B.6: In red the distribution for "real" and in blue for "fake" electrons.
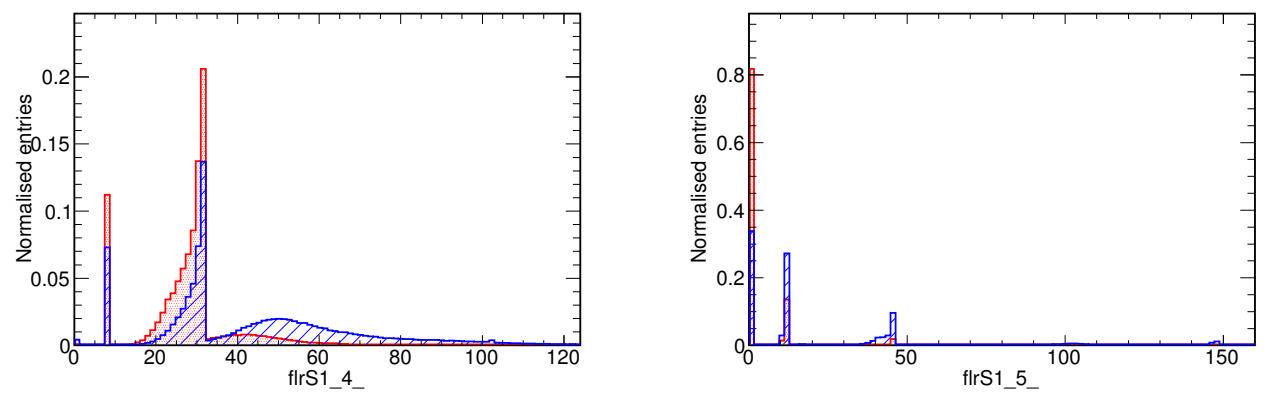

Figure B.7: In red the distribution for "real" and in blue for "fake" electrons. 

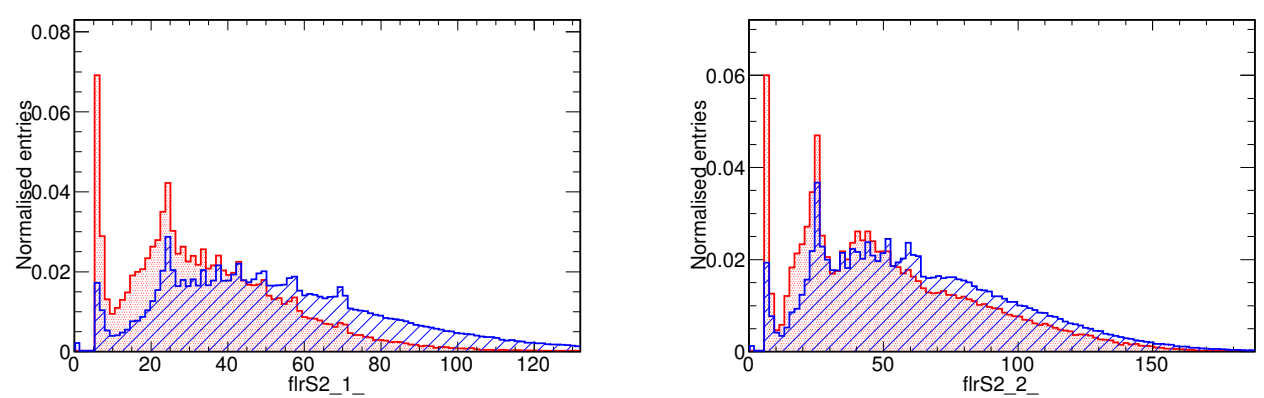

Figure B.8: In red the distribution for "real" and in blue for "fake" electrons.
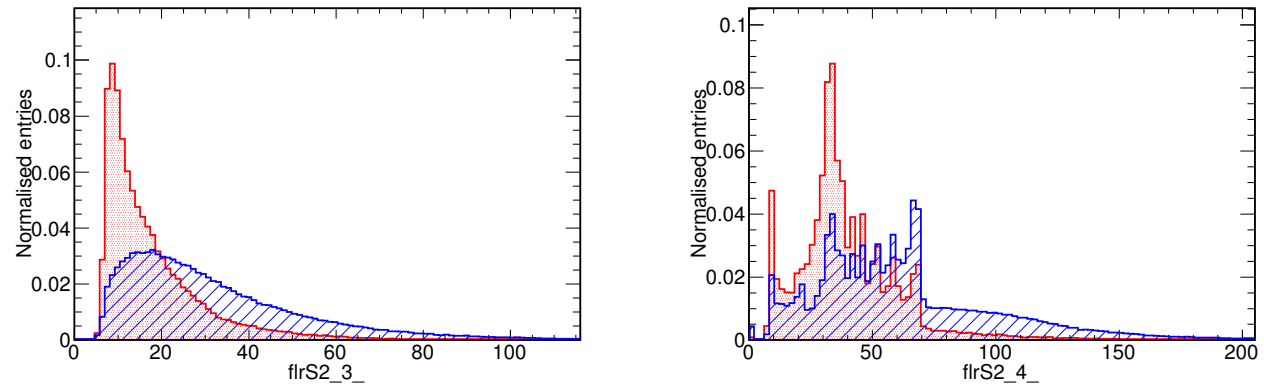

Figure B.9: In red the distribution for "real" and in blue for "fake" electrons.
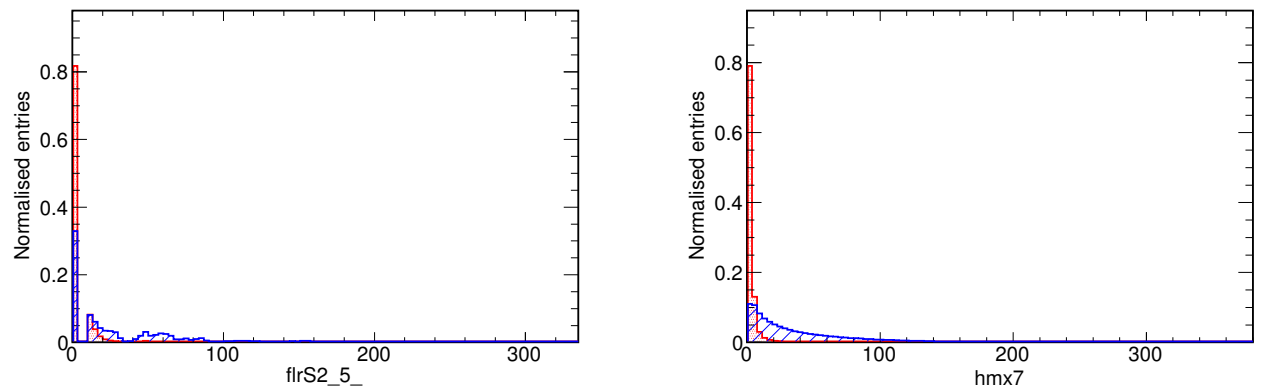

Figure B.10: In red the distribution for "real" and in blue for "fake" electrons. 

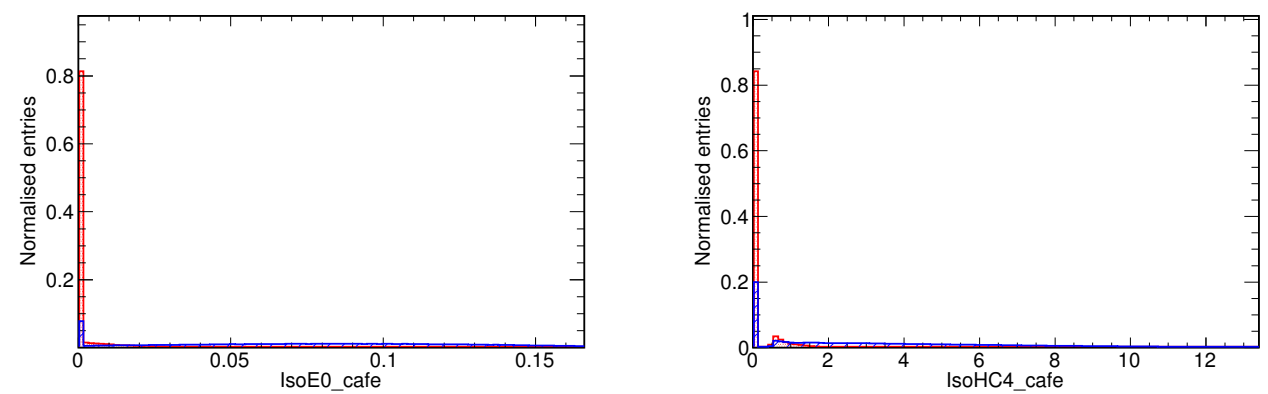

Figure B.11: In red the distribution for "real" and in blue for "fake" electrons.

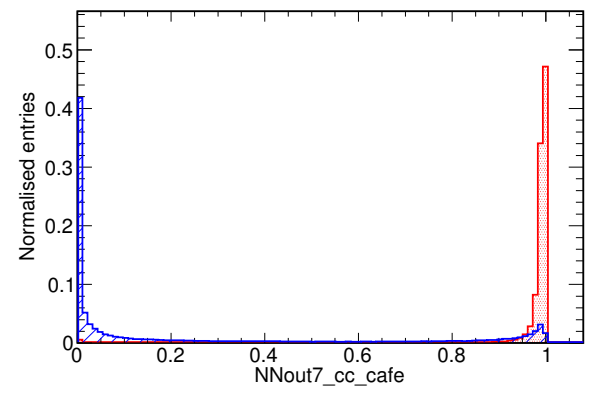

Figure B.12: In red the distribution for "real" and in blue for "fake" electrons.
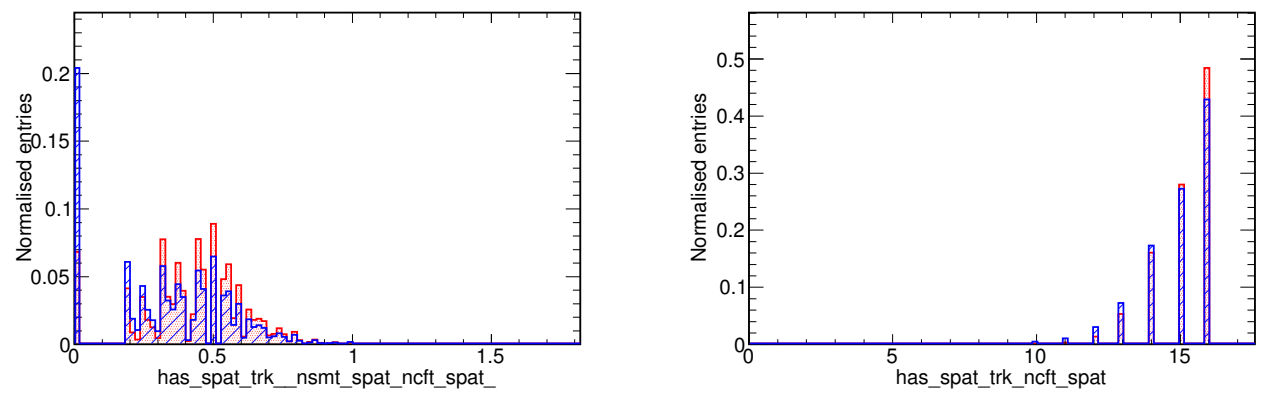

Figure B.13: In red the distribution for "real" and in blue for "fake" electrons. 

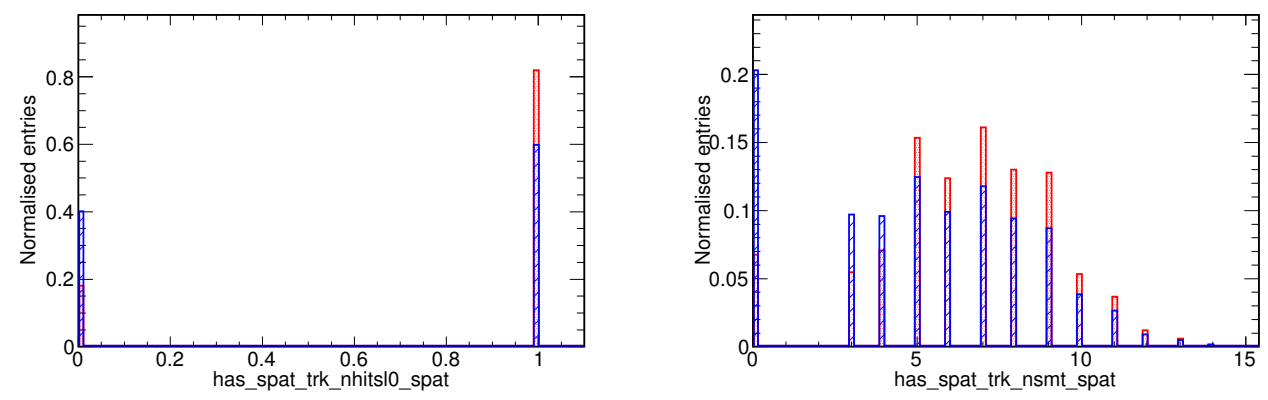

Figure B.14: In red the distribution for "real" and in blue for "fake" electrons.
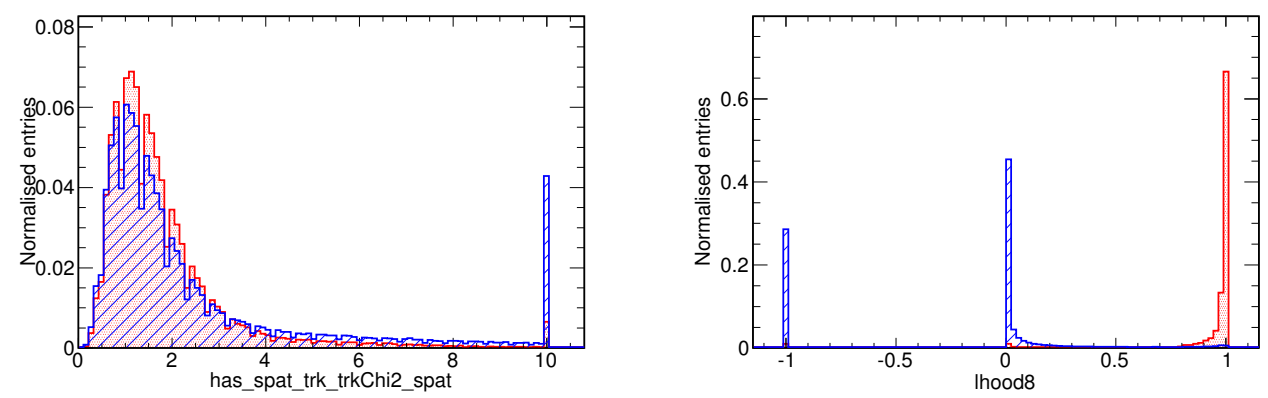

Figure B.15: In red the distribution for "real" and in blue for "fake" electrons.
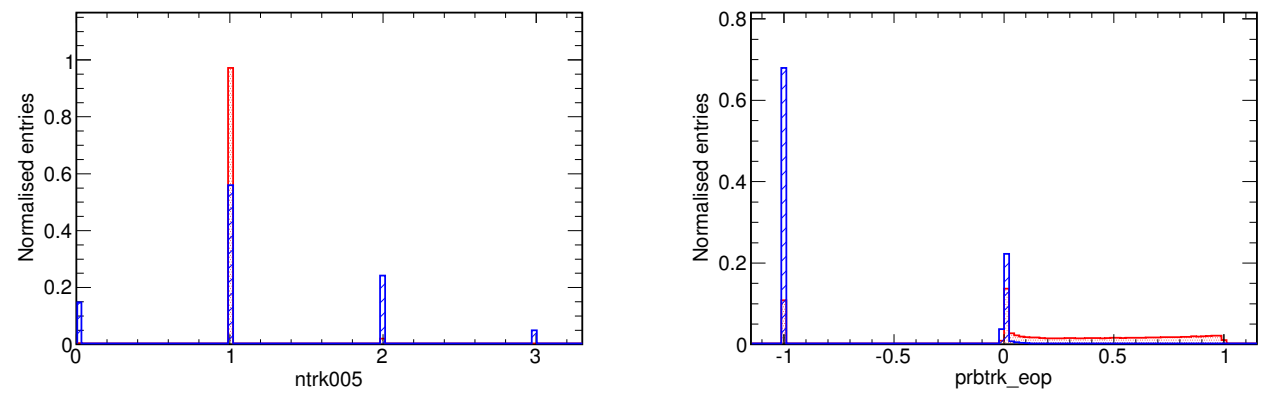

Figure B.16: In red the distribution for "real" and in blue for "fake" electrons. 

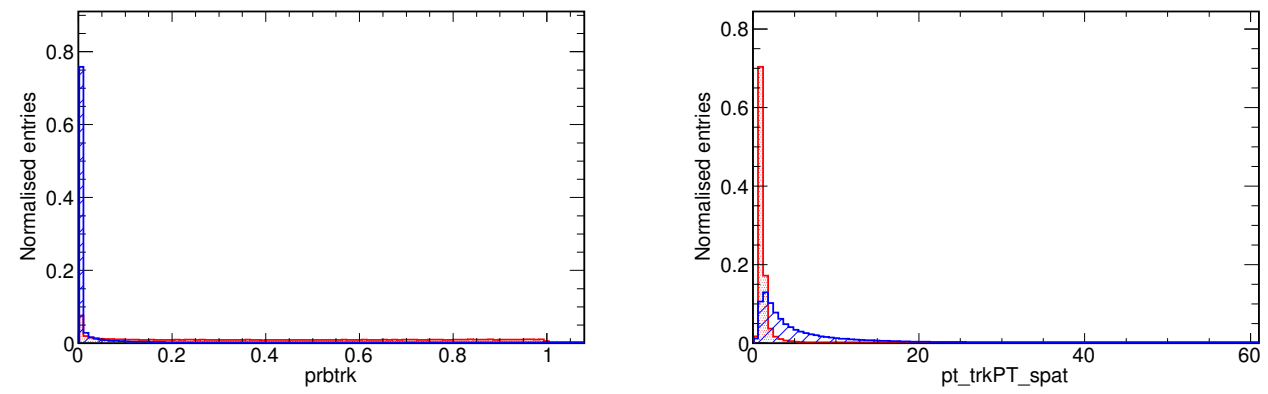

Figure B.17: In red the distribution for "real" and in blue for "fake" electrons.

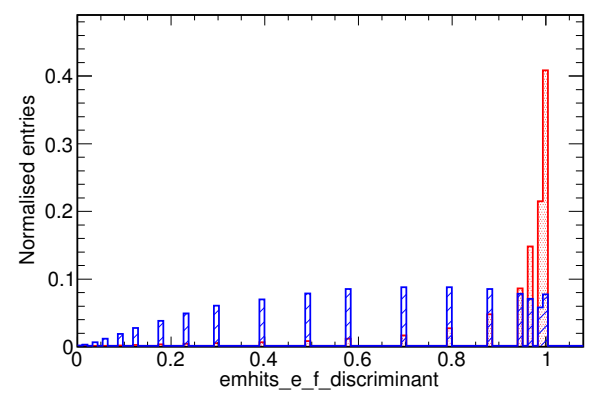

Figure B.18: In red the distribution for "real" and in blue for "fake" electrons. 

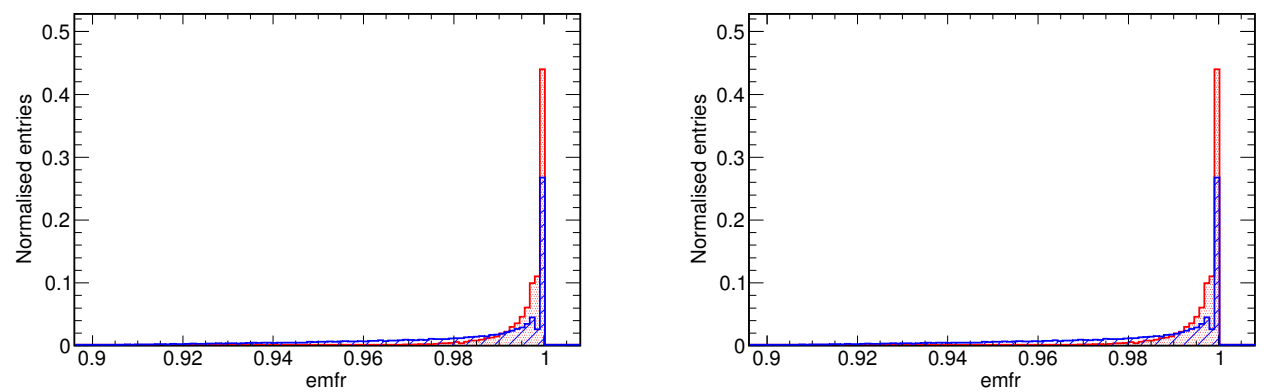

Figure B.19: In red the distribution for "real" and in blue for "fake" electrons.
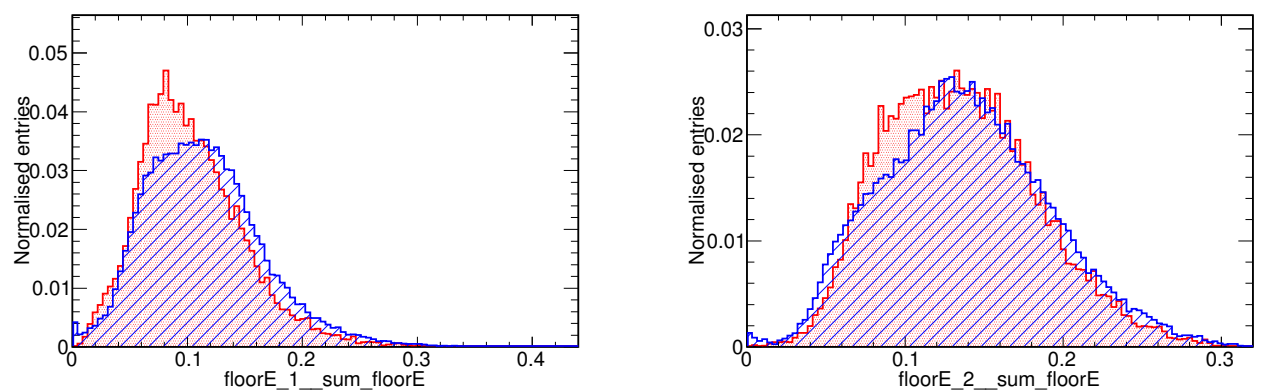

Figure B.20: In red the distribution for "real" and in blue for "fake" electrons.

\section{B.2 Endcap calorimeter variables}



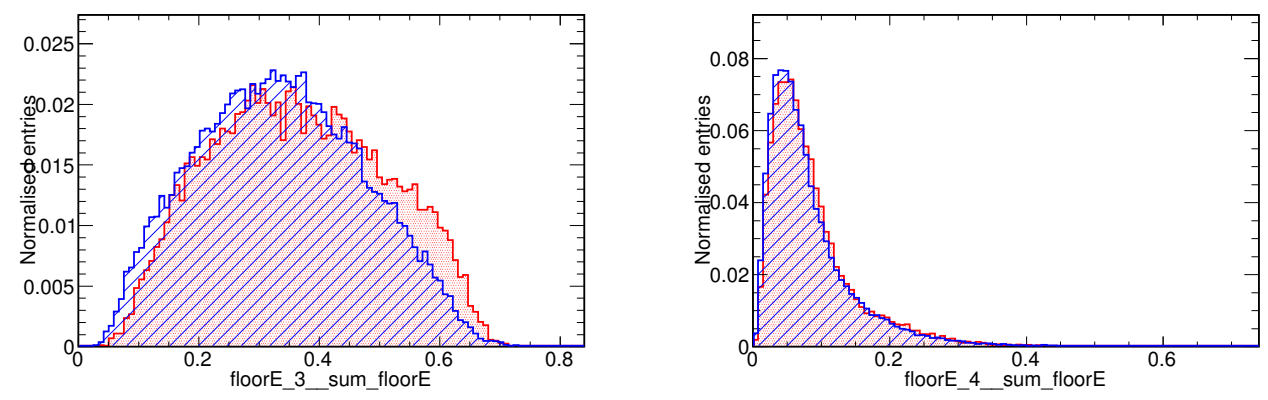

Figure B.21: In red the distribution for "real" and in blue for "fake" electrons.
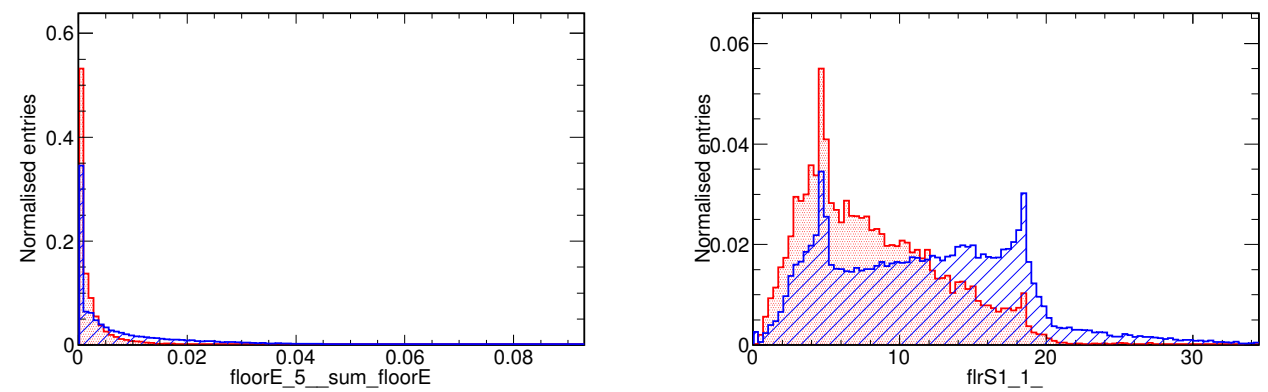

Figure B.22: In red the distribution for "real" and in blue for "fake" electrons.
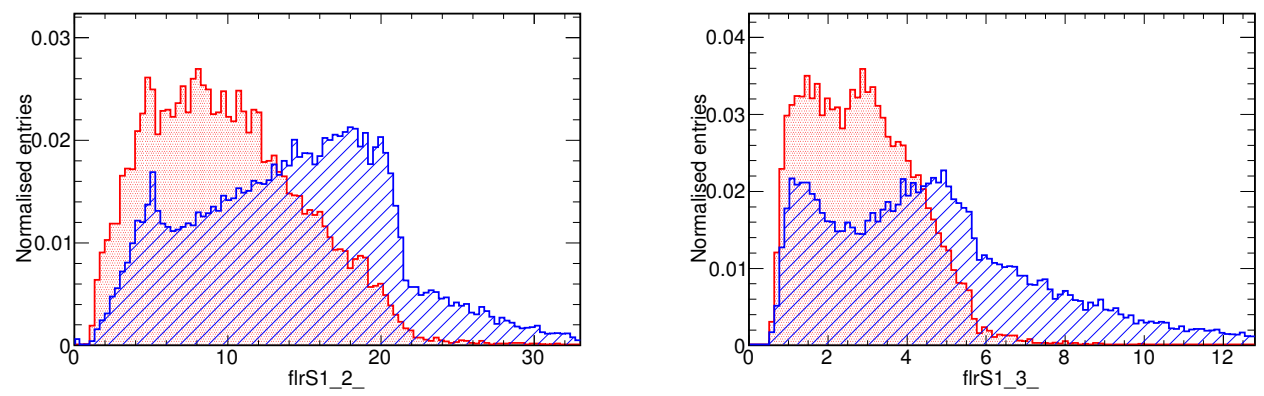

Figure B.23: In red the distribution for "real" and in blue for "fake" electrons. 

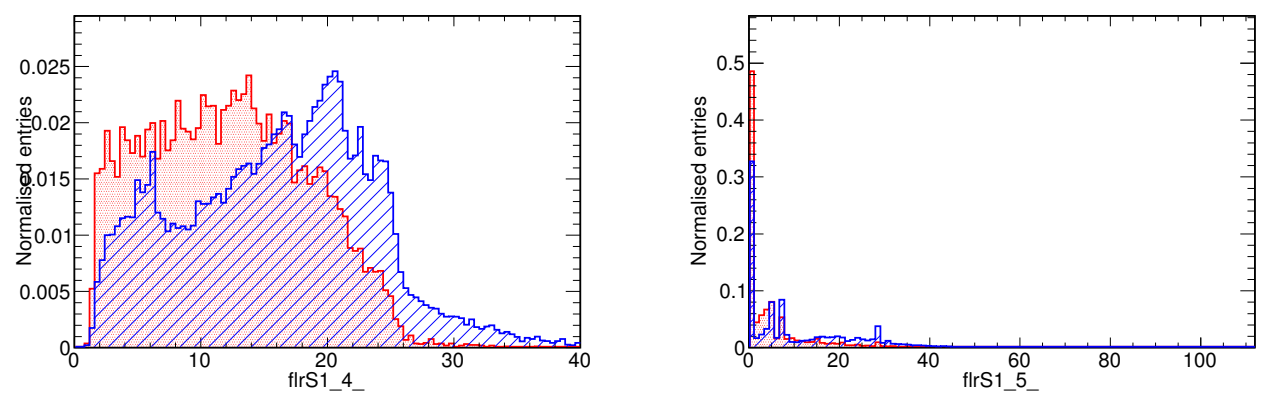

Figure B.24: In red the distribution for "real" and in blue for "fake" electrons.
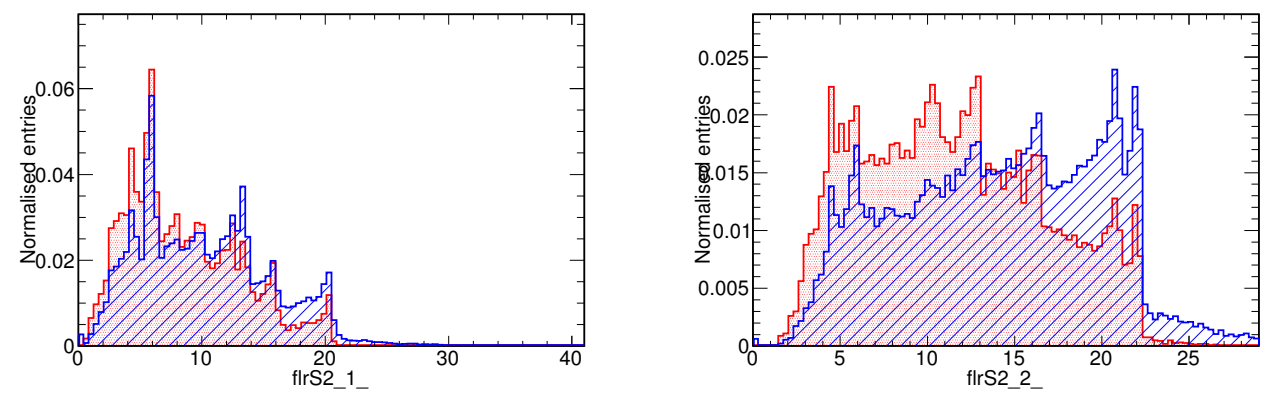

Figure B.25: In red the distribution for "real" and in blue for "fake" electrons.
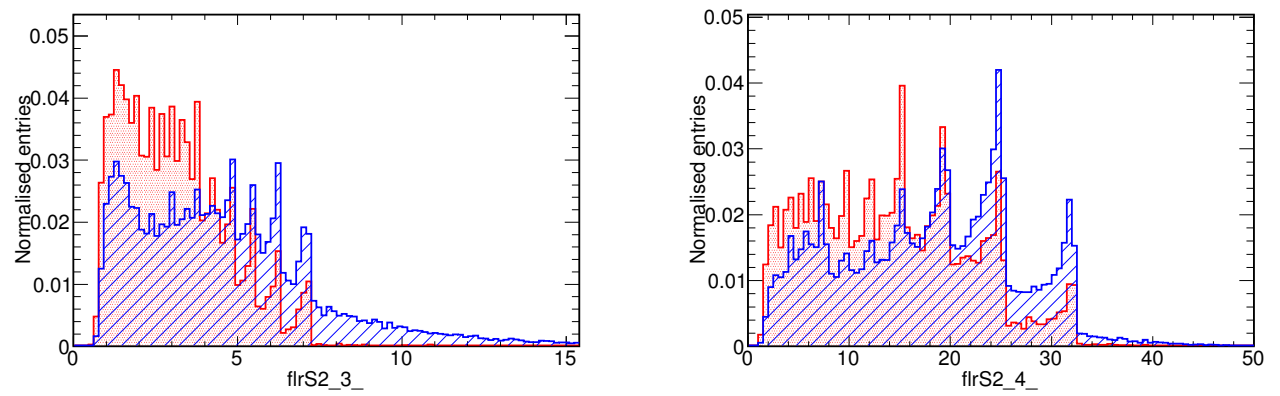

Figure B.26: In red the distribution for "real" and in blue for "fake" electrons. 

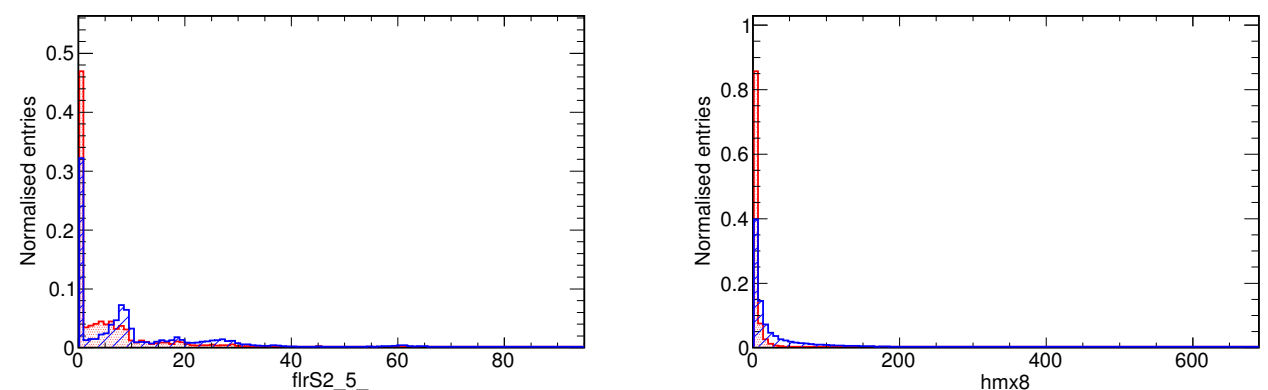

Figure B.27: In red the distribution for "real" and in blue for "fake" electrons.
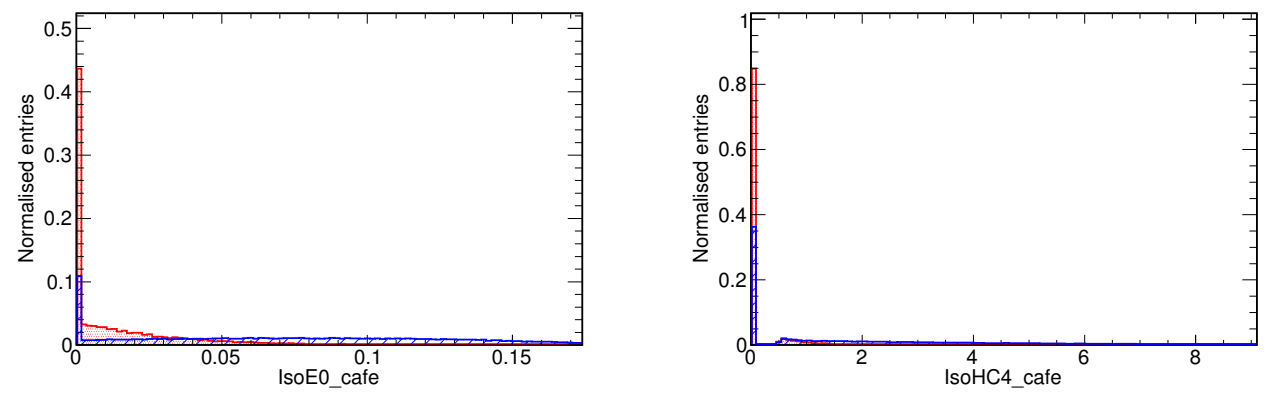

Figure B.28: In red the distribution for "real" and in blue for "fake" electrons.
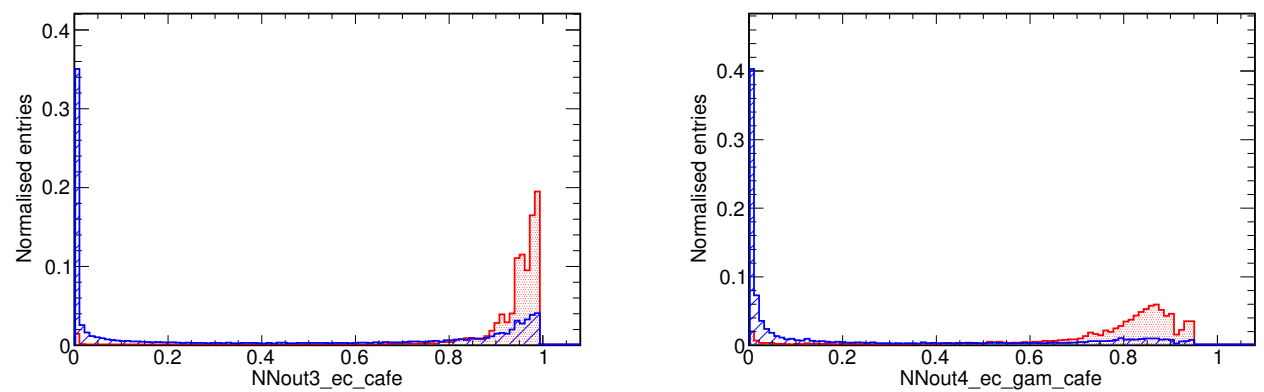

Figure B.29: In red the distribution for "real" and in blue for "fake" electrons. 

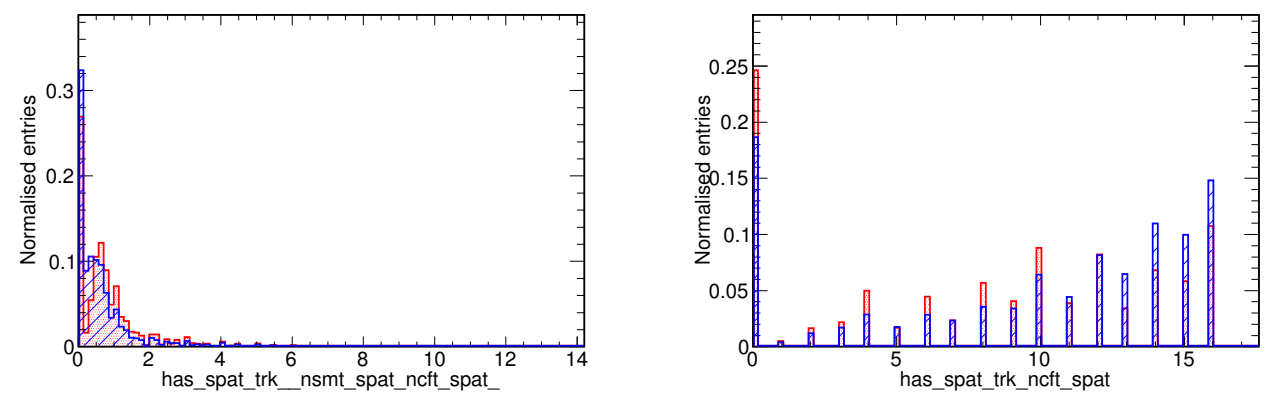

Figure B.30: In red the distribution for "real" and in blue for "fake" electrons.
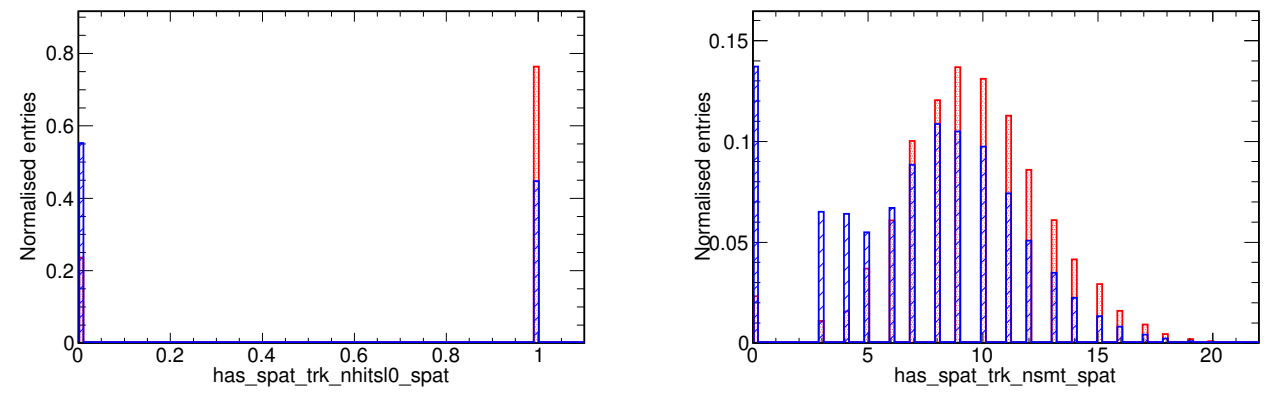

Figure B.31: In red the distribution for "real" and in blue for "fake" electrons.
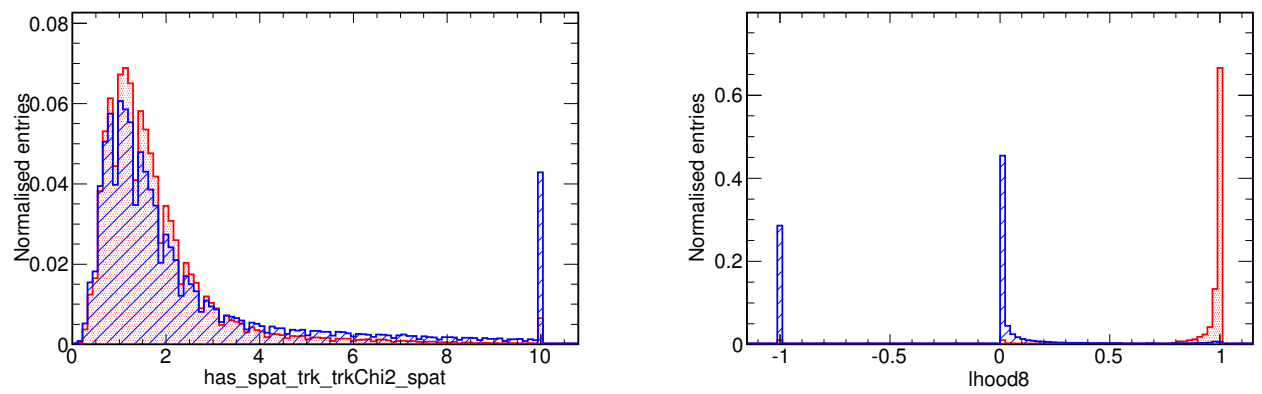

Figure B.32: In red the distribution for "real" and in blue for "fake" electrons. 

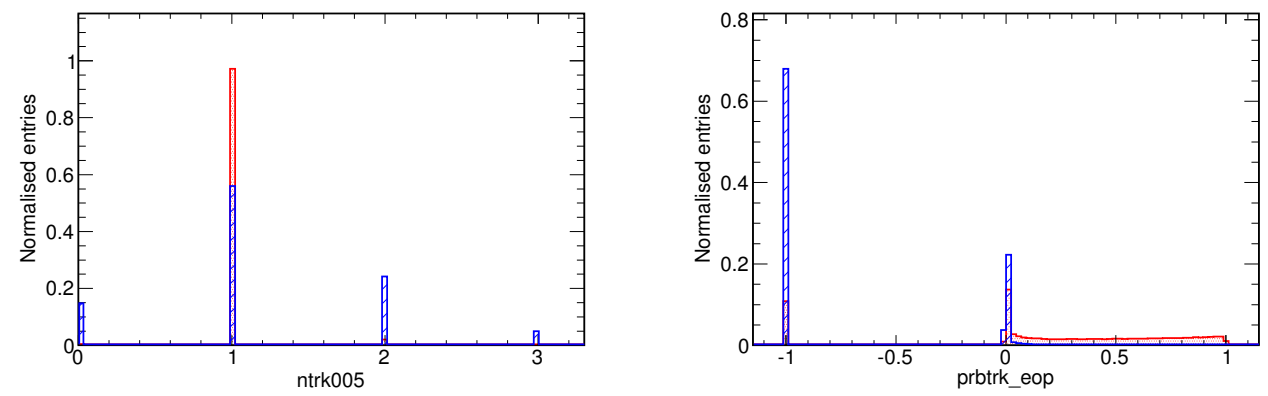

Figure B.33: In red the distribution for "real" and in blue for "fake" electrons.
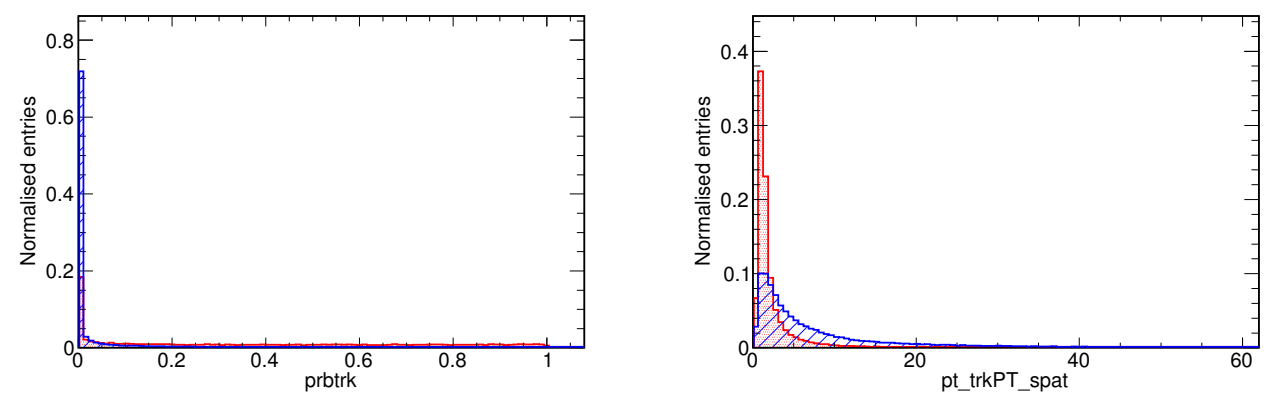

Figure B.34: In red the distribution for "real" and in blue for "fake" electrons. 


\section{Bibliography}

[1] Combination of CDF and D0 results on the mass of the top quark using up to $5.8 \mathrm{fb}-1$ of data. 2011.

[2] Georges Aad et al. Measurement of the charge asymmetry in top quark pair production in pp collisions at $\operatorname{sqrt}(\mathrm{s})=7 \mathrm{TeV}$ using the ATLAS detector. Eur.Phys.J., C72:2039, 2012.

[3] Georges Aad et al. Observation of a new particle in the search for the Standard Model Higgs boson with the ATLAS detector at the LHC. Phys.Lett., B716:1-29, 2012.

[4] Georges Aad et al. Observation of spin correlation in $t \bar{t}$ events from pp collisions at $\operatorname{sqrt}(\mathrm{s})=7 \mathrm{TeV}$ using the ATLAS detector. Phys.Rev.Lett., 108:212001, 2012.

[5] T. Aaltonen et al. Forward-Backward Asymmetry in Top Quark Production in $p \bar{p}$ Collisions at $\sqrt{s}=1.96 \mathrm{TeV}$. Phys.Rev.Lett., 101:202001, 2008 .

[6] T. Aaltonen et al. Evidence for a Mass Dependent Forward-Backward Asymmetry in Top Quark Pair Production. Phys.Rev., D83:112003, 2011.

[7] S. Abachi et al. Observation of the top quark. Phys.Rev.Lett., 74:2632$2637,1995$.

[8] Victor Mukhamedovich Abazov et al. Measurement of the top quark mass in final states with two leptons. Phys.Rev., D80:092006, 2009. 
[9] Victor Mukhamedovich Abazov et al. Determination of the width of the top quark. Phys.Rev.Lett., 106:022001, 2011.

[10] Victor Mukhamedovich Abazov et al. Forward-backward asymmetry in top quark-antiquark production. Phys.Rev., D84:112005, 2011.

[11] Victor Mukhamedovich Abazov et al. Measurement of the $t \bar{t}$ production cross section using dilepton events in $p \bar{p}$ collisions. Phys.Lett., B704:403410, 2011.

[12] Victor Mukhamedovich Abazov et al. Measurements of single top quark production cross sections and $\left|V_{t b}\right|$ in $p \bar{p}$ collisions at $\sqrt{s}=1.96 \mathrm{TeV}$. Phys.Rev., D84:112001, 2011.

[13] Victor Mukhamedovich Abazov et al. Precise measurement of the top quark mass in the dilepton channel at D0. Phys.Rev.Lett., 107:082004, 2011.

[14] Victor Mukhamedovich Abazov et al. A measurement of the $W Z$ and $Z Z$ production cross sections using leptonic final states in $8.6 \mathrm{fb}^{-1}$ of $p \bar{p}$ collisions. Phys.Rev., D85:112005, 2012.

[15] Victor Mukhamedovich Abazov et al. Search for a Narrow $t \bar{t}$ Resonance in $p \bar{p}$ Collisions at $\sqrt{s}=1.96 \mathrm{TeV}$. Phys.Rev., D85:051101, 2012.

[16] Victor Mukhamedovich Abazov et al. Search for Higgs boson production in oppositely charged dilepton and missing energy events in $p \bar{p}$ collisions at $\sqrt{s}=1.96 \mathrm{TeV}$. Phys.Rev., D86:032010, 2012.

[17] Victor Mukhamedovich Abazov et al. Search for the standard model Higgs boson in associated $W H$ production in $9.7 \mathrm{fb}^{-1}$ of $p \bar{p}$ collisions with the D0 detector. 2012.

[18] V.M. Abazov et al. The Upgraded D0 detector. Nucl.Instrum.Meth., A565:463-537, 2006. 
[19] V.M. Abazov et al. First measurement of the forward-backward charge asymmetry in top quark pair production. Phys.Rev.Lett., 100:142002, 2008.

[20] V.M. Abazov et al. Precision measurement of the ratio $\mathrm{B}(t \rightarrow$ $W b) / \mathrm{B}(t \rightarrow W q)$ and Extraction of $V_{t b}$. Phys.Rev.Lett., 107:121802, 2011.

[21] A. Abdessalam. D0 note 3745. 2000.

[22] F. Abe et al. Observation of top quark production in $\bar{p} p$ collisions. Phys.Rev.Lett., 74:2626-2631, 1995.

[23] Valentin Ahrens, Andrea Ferroglia, Matthias Neubert, Ben D. Pecjak, and Li Lin Yang. The top-pair forward-backward asymmetry beyond NLO. Phys.Rev., D84:074004, 2011.

[24] Johan Alwall, Stefan Hoche, F. Krauss, N. Lavesson, L. Lonnblad, et al. Comparative study of various algorithms for the merging of parton showers and matrix elements in hadronic collisions. Eur.Phys.J., C53:473-500, 2008.

[25] R. Angstadt et al. The Layer 0 Inner Silicon Detector of the D0 Experiment. Nucl.Instrum.Meth., A622:298-310, 2010.

[26] J. Beringer et al. Review of Particle Physics (RPP). Phys.Rev., D86:010001, 2012.

[27] W. Bernreuther, A. Brandenburg, Z.G. Si, and P. Uwer. Top quark pair production and decay at hadron colliders. Nucl.Phys., B690:81-137, 2004 .

[28] Werner Bernreuther and Zong-Guo Si. Distributions and correlations for top quark pair production and decay at the Tevatron and LHC. Nucl.Phys., B837:90-121, 2010.

[29] Werner Bernreuther and Zong-Guo Si. Top quark and leptonic charge asymmetries for the Tevatron and LHC. Phys.Rev., D86:034026, 2012. 
[30] M. Besancon et al. D0 note 6027. 2010.

[31] Ikaros I.Y. Bigi, Yuri L. Dokshitzer, Valery A. Khoze, Johann H. Kuhn, and Peter M. Zerwas. Production and Decay Properties of Ultraheavy Quarks. Phys.Lett., B181:157, 1986.

[32] Gerald C. Blazey, Jay R. Dittmann, Stephen D. Ellis, V. Daniel Elvira, K. Frame, et al. Run II jet physics. pages 47-77, 2000.

[33] Arnd Brandenburg, Z.G. Si, and P. Uwer. QCD corrected spin analyzing power of jets in decays of polarized top quarks. Phys.Lett., B539:235$241,2002$.

[34] Nicola Cabibbo. Unitary Symmetry and Leptonic Decays. Phys.Rev.Lett., 10:531-533, 1963.

[35] P. Calfayan et al. D0 note 5157. 2000.

[36] John M. Campbell and R. Keith Ellis. An Update on vector boson pair production at hadron colliders. Phys.Rev., D60:113006, 1999.

[37] Serguei Chatrchyan et al. Measurement of the charge asymmetry in topquark pair production in proton-proton collisions at $\operatorname{sqrt}(\mathrm{s})=7 \mathrm{TeV}$. Phys.Lett., B709:28-49, 2012.

[38] Serguei Chatrchyan et al. Observation of a new boson at a mass of 125 GeV with the CMS experiment at the LHC. Phys.Lett., B716:30-61, 2012 .

[39] Andrzej Czarnecki, Marek Jezabek, and Johann H. Kuhn. LEPTON SPECTRA FROM DECAYS OF POLARIZED TOP QUARKS. Nucl.Phys., B351:70-80, 1991.

[40] Abdelhak Djouadi. The Anatomy of electro-weak symmetry breaking. I: The Higgs boson in the standard model. Phys.Rept., 457:1-216, 2008.

[41] F. Englert and R. Brout. Broken Symmetry and the Mass of Gauge Vector Mesons. Phys.Rev.Lett., 13:321-323, 1964. 
[42] Gary J. Feldman and Robert D. Cousins. A Unified approach to the classical statistical analysis of small signals. Phys.Rev., D57:3873-3889, 1998.

[43] Y. Fukuda et al. Evidence for oscillation of atmospheric neutrinos. Phys.Rev.Lett., 81:1562-1567, 1998.

[44] Philippe Gris. D0 note 5400. 2007.

[45] Alexander Grohsjean. Measurement of the top quark mass in the dilepton final state using the matrix element method. 2008.

[46] G.S. Guralnik, C.R. Hagen, and T.W.B. Kibble. Global Conservation Laws and Massless Particles. Phys.Rev.Lett., 13:585-587, 1964.

[47] P. Haefner et al. D0 note 4818. 2005.

[48] F. Halzen, P. Hoyer, and C.S. Kim. Forward-backward asymmetry of hadroproduced heavy quarks in QCD. Phys.Lett., B195:74, 1987.

[49] Amnon Harel. D0 note 4919. 2005.

[50] Robert M. Harris, Christopher T. Hill, and Stephen J. Parke. Crosssection for topcolor Z-prime(t) decaying to t anti-t: Version 2.6. 1999.

[51] Peter W. Higgs. Broken Symmetries and the Masses of Gauge Bosons. Phys.Rev.Lett., 13:508-509, 1964.

[52] Andreas Hocker, J. Stelzer, F. Tegenfeldt, H. Voss, K. Voss, et al. TMVA - Toolkit for Multivariate Data Analysis. PoS, ACAT:040, 2007.

[53] M. Hohlfeld. D0 note 5783. 2008.

[54] A. Juste et al. http://www-d0.fnal.gov/phys_id/jes/public_ RunIIa.

[55] A. Juste et al. D0 note 5382. 2007.

[56] A. Khanov. HTF: histogramming method for finding tracks. The algorithm description. D0 note 3778, 2000. 
[57] Nikolaos Kidonakis. Single top production at the Tevatron: Threshold resummation and finite-order soft gluon corrections. Phys.Rev., D74:114012, 2006.

[58] Makoto Kobayashi and Toshihide Maskawa. CP Violation in the Renormalizable Theory of Weak Interaction. Prog.Theor.Phys., 49:652-657, 1973.

[59] David Krohn, Tao Liu, Jessie Shelton, and Lian-Tao Wang. A Polarized View of the Top Asymmetry. Phys.Rev., D84:074034, 2011.

[60] Louis Lyons, Duncan Gibaut, and Peter Clifford. How to combine correlated estimates of a single physical quantity. Nucl.Instrum.Meth., A270:110, 1988.

[61] Gregory Mahlon and Stephen J. Parke. Improved spin basis for angular correlation studies in single top quark production at the Tevatron. Phys.Rev., D55:7249-7254, 1997.

[62] Gregory Mahlon and Stephen J. Parke. Maximizing spin correlations in top quark pair production at the Tevatron. Phys.Lett., B411:173-179, 1997.

[63] John Marriner. Stochastic cooling overview. Nucl.Instrum.Meth., A532:11-18, 2004

[64] Sven Moch and Peter Uwer. Theoretical status and prospects for topquark pair production at hadron colliders. Phys.Rev., D78:034003, 2008.

[65] P. Nason, S. Dawson, and R. Keith Ellis. The Total Cross-Section for the Production of Heavy Quarks in Hadronic Collisions. Nucl.Phys., B303:607, 1988.

[66] J. Neyman. Phil. Trans. Royal Soc. London, 236:333-380, 1937.

[67] M. Owen and M. Hohlfeld. D0 note 5409. 2007. 
[68] Ariel G. Schwartzman. Measurement of the B+- lifetime and top quark identification using secondary vertex b-tagging. $\mathrm{PhD}$ thesis, 2004. 